NIST Technical Note 2140

\title{
AWS-3 LTE Impacts on Aeronautical Mobile Telemetry
}

\author{
William F. Young \\ Duncan A. McGillivray \\ Adam J. Wunderlich \\ Mark A. Krangle \\ Jack G. Sklar \\ Aric W. Sanders \\ M. Keith Forsyth \\ Mark Lofquist \\ Daniel G. Kuester
}

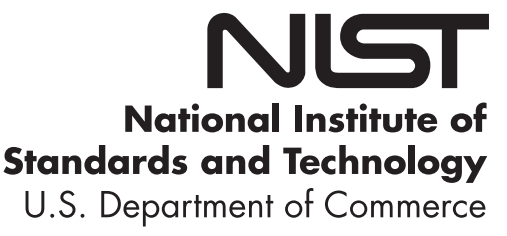


NIST Technical Note 2140

\section{AWS-3 LTE Impacts on Aeronautical Mobile Telemetry}

William F. Young MITRE Corp.

Duncan A. McGillivray

Adam J. Wunderlich

Communications Technology Laboratory

NIST

Mark A. Krangle

MITRE Corp.

Jack G. Sklar

Aric W. Sanders

M. Keith Forsyth

Communications Technology Laboratory

NIST

Mark Lofquist

MITRE Corp.

Daniel G. Kuester

Communications Technology Laboratory

NIST

This publication is available free of charge from:

https://doi.org/10.6028/NIST.TN.2140

February 2021

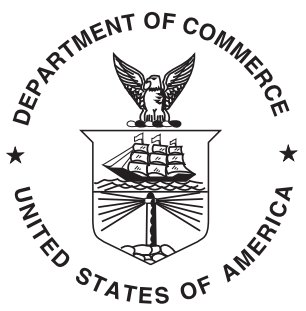

U.S. Department of Commerce Wynn Coggins, Acting Secretary

National Institute of Standards and Technology James K. Olthoff, Performing the Non-Exclusive Functions and Duties of the Under Secretary of Commerce for Standards and Technology \& Director, National Institute of Standards and Technology 


\section{AWS-3 LTE Impacts on Aeronautical Mobile Telemetry}

William F. Young, Duncan A. McGillivray, Adam J. Wunderlich, Mark A. Krangle, Jack G. Sklar, Aric W. Sanders, M. Keith Forsyth, Mark Lofquist, Daniel G. Kuester

NASCTN Project Manager: Fabio C.S. Da Silva NASCTN Program Manager: Melissa M. Midzor

February 24, 2021
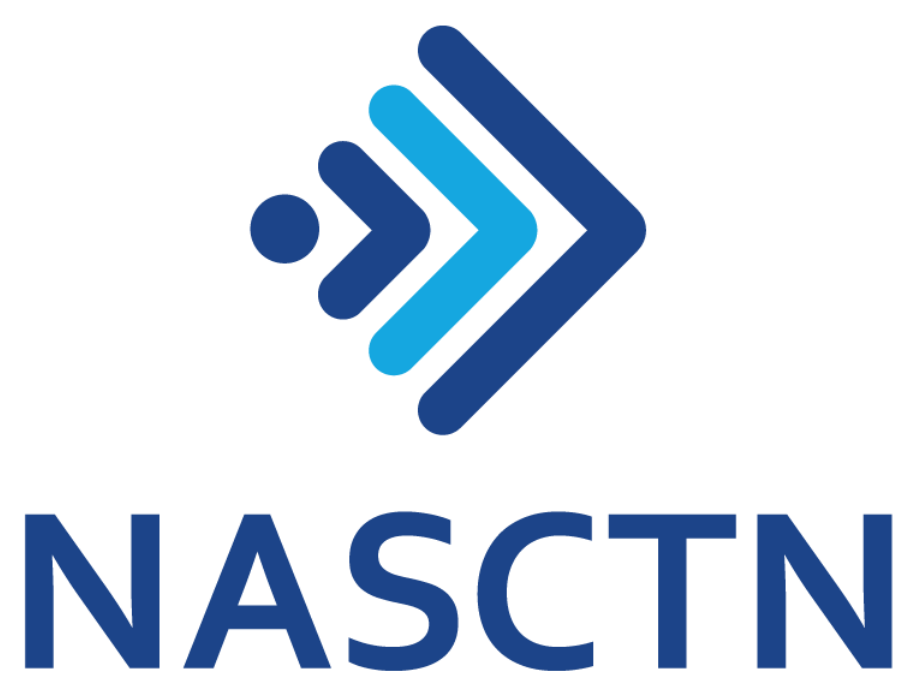

National Advanced Spectrum and Communications Test Network 


\section{DISCLAIMER}

Certain commercial entities, equipment, or materials may be identified in this document in order to describe an experimental procedure or concept adequately. Such identification does not imply any recommendation or endorsement by National Institute of Standards and Technology (NIST) or National Advanced Spectrum and Communications Test Network (NASCTN), nor is it intended to imply that the materials or equipment identified are necessarily the best available for the purpose.

National Institute of Standards and Technology Technical Note 2140 Natl. Inst. Stand. Technol. Tech. Note 2140, 150 pages (February 2021) 


\section{Preface}

The work described herein was performed as a result of a spectrum sharing project request submitted to the National Advanced Spectrum and Communications Test Network (NASCTN). NIST is a founding charter member of NASCTN. This work was sponsored by Edwards Airforce Base, and conducted by NIST with support by NTIA, NASA, and MITRE. A summary of each organization's technical contributions follows.

The project underwent formal screening and approval by the NASCTN Steering Committee. A description of NASCTN and listing of Charter Members at the time of publication of this report follows. 


\section{National Advanced Spectrum and Communications Test Network (NASCTN)}

The mission of the National Advanced Spectrum and Communications Test Network (NASCTN) is to provide, through its members, a network for robust test processes and validated measurement data necessary to develop, evaluate and deploy spectrum sharing technologies that can improve access to the spectrum by both federal agencies and non-federal spectrum users.

NASCTN is a member organization under a charter agreement. Members

- Facilitate and coordinate work with federal, academic, and industry spectrum users to rapidly and cooperatively facilitate spectrum sharing and co-existence studies;

- Work as a partnership to address the interests and equities of all spectrum stakeholders in a fair, equitable, and non-preferential manner; and

- Through sharing of technical resources, with consideration for cost, provide liaison and support to coordinate and leverage existing national capabilities supporting government, academic, and industry testing and evaluation known to improve and expedite spectrum sharing and coexistence.

Charter members at the time of publication of this report are (in alphabetical order):

- Department of Defense Chief Information Officer (DoD CIO)

- National Aeronautics and Space Administration (NASA)

- National Institute of Standards and Technology (NIST)

- National Oceanic and Atmospheric Administration (NOAA)

- National Science Foundation (NSF)

- National Telecommunications and Information Administration (NTIA)

NIST hosts the NASCTN capability at the Department of Commerce Boulder Laboratories in Boulder, Colorado. 


\section{Technical Contributors}

\begin{tabular}{lll}
\hline \hline Contributor & Organization & Primary Contribution Areas \\
\hline Evan Briggs & MITRE & Testbed automation instrument driver and database development \\
Victor Haley & MITRE & Testbed automation software support \\
Ashton Knight & MITRE & Testbed automation system diagnostic support \\
Mark Krangle & MITRE & Bedford testbed RF development and Bedford test execution lead \\
Shawn Lefebre & MITRE & Testbed automation framework and instrument driver development \\
Mark Lofquist & MITRE & Frequency spectrum policies and test objectives \\
Tim Mull & MITRE & Pilot study and data analysis support \\
Adam Paranay & MITRE & Testbed RF development \\
William Young & MITRE & Project technical lead \\
Jacob Johnson & MITRE (Intern) & Adjacent band waveform conditioning support \\
Matt Puentes & MITRE (Intern) & Testbed automation software support \\
Nevan Shattuck & MITRE (Intern) & Bedford testbed lab support \\
Kenneth Dudley & NASA LARC & Telemetry systems and applications \\
Claude (Lee) Joyce & NASA LARC & Telemetry systems and applications \\
Michael K. Forsyth & NIST NASCTN & Automation refinement and Boulder test execution lead \\
Daniel Kuester & NIST & Noise figure measurement theory and design \\
Duncan McGillivray & NIST NASCTN & Test execution lead, testbed RF design, experiment calibration \\
Jack Sklar & NIST NASCTN & Data analysis and data publication \\
Adam Wunderlich & NIST NASCTN & Data analysis and visualization lead, experimental design \\
Aric Sanders & NIST NASCTN (Assoc.) & Testbed automation refinement, data analysis and visualization \\
Frank Sanders & NTIA ITS & RF and radar systems \\
Eric Nelson & NTIA ITS & Spectrum measurements and signal analysis \\
Kenneth Brewster & NTIA ITS & Field measurements and calibrations \\
\hline
\end{tabular}




\section{Acknowledgments}

We would like to thank the many additional individuals and organizations that made valuable contributions over the course of this project. Thanks to the contributions from Charmaine Franck at the National Aeronautics and Space Administation (NASA) Langley Research Center (LARC) that enabled the inclusion of NASA telemetry experts throughout the testing process. Additional thanks to Dr. Keith Hartley of the MITRE Corporation and Irena Stevens of University of Colorado at Boulder for providing project management support. We acknowledge and thank Rebecca Dorch of the National Telecommunications and Information Administration (NTIA) Institute for Telecommunication Sciences (ITS) for her efforts to enable subject matter expertise in spectrum measurements to participate in this test campaign.

We also thank Thomas O'Brien of the Test Resource Management Center and Thomas Taylor of the Department of Defense Chief Information Office for their resources.

Furthermore, We would like to acknowledge the efforts of the test plan development team and thank those who participated in the public comment period for the test plan.

Also, the technical team thanks the National Advanced Spectrum and Communications Test Network (NASCTN) program manager, Dr. Melissa Midzor, former NASCTN project manager, Dr. Fabio da Silva, and the NASCTN administrative officers, Linda Derr, and Amanda Hyman for their efforts, which made project success possible.

Finally, thanks to Barbara Wheaton and Kenneth "Kip" Temple for their guidance and feedback throughout the project evolution from the initial NASCTN proposal to the results presented here. Their direction and insights were critical to the body of work on aeronautical mobile telemetry (AMT) susceptibility to advanced wireless services 3 (AWS-3) emissions. 


\section{Executive Summary}

Representatives from Edwards Air Force Base (EAFB) submitted a proposal to National Advanced Spectrum and Communications Test Network (NASCTN) to measure the impacts of long-term evolution (LTE) user equipment (UE) emissions in the United States advanced wireless services 3 (AWS-3) frequency band $(1755 \mathrm{MHz}-1780 \mathrm{MHz}$ ) and aeronautical mobile telemetry (AMT) systems in the adjacent L band $(1780 \mathrm{MHz}-1850 \mathrm{MHz})$. The primary goal of the proposal is to investigate potential impacts of LTE adjacent band emissions to the AMT systems.

The NASCTN steering committee accepted the EAFB proposal, and the test plan development team delivered and circulated a test and metrology plan for public comment. Upon finalizing the plan based on the received feedback, the test execution began in March of 2019. The test plan, comments matrix and other supporting material are available on the NASCTN website ${ }^{1}$.

The objective of this NASCTN effort is to design, demonstrate, and validate a test methodology to measure the impacts of LTE UE emissions on the adjacent AMT band. Impacts are assessed by collecting and analyzing performance measures, such as bit error rate (BER) and $E_{b} / N_{0}$, generally referred to as key performance indicators (KPIs). The validation process includes the capture and analysis for multiple adjacent band emissions (ABE) and AMT transmission combinations and conditions. The analysis here centers around BER behavior with and without the presence of ABE.

A key part of the experiments is determining how close the AMT center frequency can be located to the band-edge of $1780 \mathrm{MHz}$ for a variety of AMT modulation formats and bit rates, with several representative waveforms in the adjacent band. This information is helpful in establishing and confirming frequency edge back-off recommendations in the Inter-Range Instrumentation Group (IRIG) 106 Standard [1]. In addition, the concept of Gaussian "equivalent in-band noise" is proposed to aid interpretation of the non-linear effects in AMT receiver response caused by some of the LTE ABE waveforms.

The design, development, execution, and data analysis of this test support several general and specific observations.

- Proper consideration of out of band emissionss (OOBEs) is important to understanding the impacts due to adjacent band signals. Simulations or emulations of ABEs do not generally represent real-world OOBEs, and those differences can have a significant impact on the adjacent band receiver.

- Automated testing allows for detailed and highly-controlled investigations of the impacts due to ABEs. In this case, the automation allows for the comparison of impacts from a wide variety of waveforms. Note that running a similar testing campaign in the field will, in general, result in higher measurement uncertainties than using a laboratory settings such as in this investigation.

- Two ABE waveforms with nearly equivalent average powers but with seemingly inconsequential differences in OOBE behavior can cause very different impacts on the AMT system. This is clearly evident in the frequency offset results in Section 5.2.2.

- The main experiment frequency back-off results suggest that the IRIG back-off calculations work for most of the test ABE types. In the main experiment results, the only ABE type and AMT modulation combinations that exceed a BER $=10^{-5}$ (i.e., exceed the desired threshold), are the multi-UE at azimuth of $198^{\circ}$ ( $37 \mathrm{MHz}$ filter) for shaped offset quadrature-phase shift keying (SOQPSK) bit rates of $5 \mathrm{Mbps}$ and $10 \mathrm{Mbps}$, and the multi-UE at azimuth of $198^{\circ}$ (37 MHz filter) and a single-UE with $100 \mathrm{RB}$ (37 MHz filter) for an Advanced Range Telemetry continuous phase modulation (ARTM CPM) bit rate of 5 Mbps.

- For the single UE cases tested, the ABE OOBE profile correlates to the LTE resource block (RB) allocations.

\footnotetext{
${ }^{1}$ NASCTN Project page: https://www.nist.gov/programs-projects/aws-3-lte-impacts-amt
} 
- The impacts due to ABEs are dominated by the frequency overlap of the intermediate frequency (IF) filter bandwidth and the adjacent band LTE and LTE OOBE activity.

- The use of the ABE root mean square (RMS) power value in the signal to adjacent band emitter power ratio (SABE) calculations can lead to misrepresentation in predicting impact of a particular waveform, when compared to the no ABE condition ${ }^{2}$.

- The concept of an equivalent in-band white Gaussian noise is proposed as a way to interpret the impacts on $E_{b} / N_{0}$. These results demonstrate non-linear effects across a range of BER values that fall in the desired operating region of better than BER $=10^{-5}$ for several of the tested ABE and AMT signal combinations.

- In-field measurements serve as surrogates and may not replicate the exact adjacent band deployment parameters (e.g., $20 \mathrm{MHz}$ versus $10 \mathrm{MHz}$ allocations, LTE versus 5G technology). In general, deployments that allow increased RB activity or power at the band edge closest to AMT may require an increase in the band edge frequency offset.

- As demonstrated in Appendix D scatter plots, receiver-reported $E_{b} / N_{0}$ is not a reliable indicator of BER performance in the presence of ABE. Since $E_{b} / N_{0}$ is a ratio of in-band quantities, this observation is expected.

The investigation and test methodology demonstration takes place on two testbeds, one located at the MITRE facility in Bedford, MA, and the other at the NASCTN facility in Boulder, CO. The benefits of running multiple testbeds are the ability to validate and trouble shoot test runs, as well as increase the confidence in the reproducibility of results. Leveraging two testbeds allows the development of the automation code and framework while simultaneously prototyping testing methodologies and protocols (i.e., the main and frequency offset experiments). The two testbeds allow the concurrent investigation of concepts such as the Gaussian equivalent in band noise on the NASCTN testbed without disrupting the Bedford testbed generation of the primary experiment data.

Finally, the use of automated testbeds improves the rigor of the measurement process by allowing collection of statistically rich data sets and the ability to investigate behaviors that might initially appear anomalous. Accompanying these measurements is rigorous uncertainty analysis that includes an uncertainty budget and calculation of overall measurement uncertainty. The combination of automation and remote access allows testing to continue with very minimal on-site interaction with the testbed and measurement equipment.

The complete set of test data for this project is publicly available at https://doi.org/10.18434/M32279.

\footnotetext{
${ }^{2}$ Section 1.4 of the ITU-R SM.1541-6 defines the adjacent band power ratio as the ratio between a transmitter mean power and the mean power in the adjacent frequency band. However, it should not be assumed that adjacent band interference measurements are commonly referenced to RMS power.
} 


\section{Contents}

Technical Contributors ................................ . . . . . . .

Acknowledgments . . . . . . . . . . . . . . . . . . . . . . . . . vi

Executive Summary . . . . . . . . . . . . . . . . . . . . . . . . vii

List of Acronyms . . . . . . . . . . . . . . . . . . . . . . . . . . xii

List of Figures . . . . . . . . . . . . . . . . . . . . . . . . . . . X

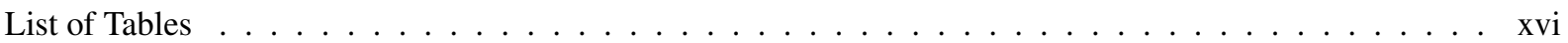

1 Introduction 1

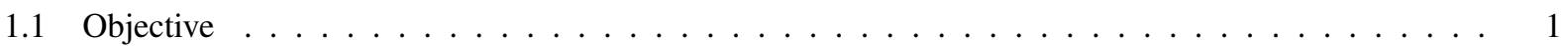

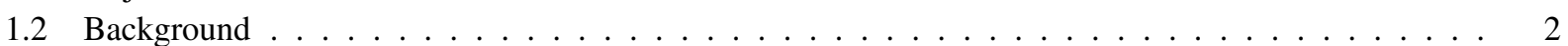

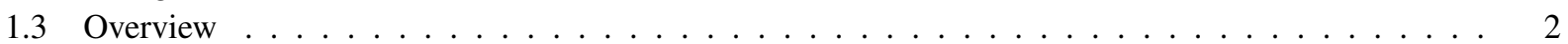

2 Experimental Design 4

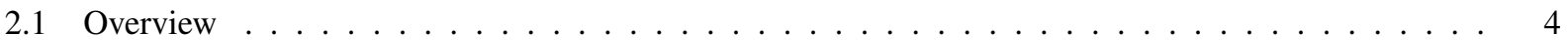

2.2 Pilot Study . . . . . . . . . . . . . . . . . . . . . . . . . 4

2.3 Main Characterization Experiment . . . . . . . . . . . . . . . . . . . . . . 5

2.4 Frequency Offset Experiment . . . . . . . . . . . . . . . . . . . . . . . . . 6

2.5 Supplemental Experiments . . . . . . . . . . . . . . . . . . . . . 7

2.5.1 Side Experiment A: Equalizer . . . . . . . . . . . . . . . . . . . . 7

2.5.2 Side Experiment B: Space-Time Coding . . . . . . . . . . . . . . . . . . . 7

2.5.3 Side Experiment C: Excess Noise . . . . . . . . . . . . . . . . . . . . 7

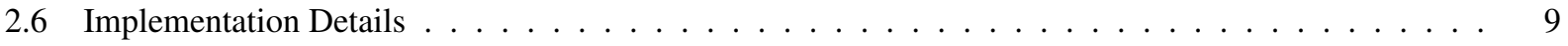

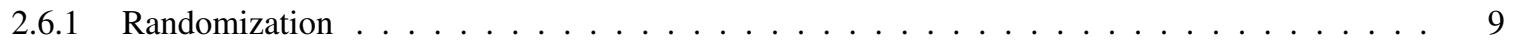

2.6.2 ABE Waveform Selection and Conditioning . . . . . . . . . . . . . . . . . . 9

3 Test Implementation and Execution 11

3.1 Overview ................................11

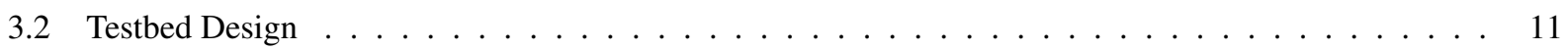

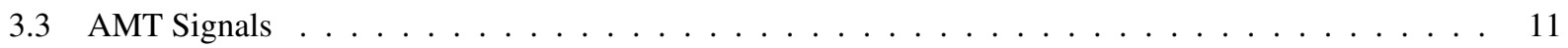

3.3.1 Receiver IF Filter Response . . . . . . . . . . . . . . . . . . . . . . . . . . . . . . 12

3.3.2 Center Frequency Selection . . . . . . . . . . . . . . . . . . . . . . 13

3.3.2.1 AMT Occupied Bandwidth and Minimum Test Frequency . . . . . . . . . . . . . 13

3.3.3 IRIG Band Edge Backoff Frequencies . . . . . . . . . . . . . . . . . . . . . . . . 14

3.4 Adjacent Band Emissions . . . . . . . . . . . . . . . . . . . . . . . . . . . . . . 14

3.5 Testbed Differences for AMT and ABE Test Frequencies . . . . . . . . . . . . . . . . . . . . 15

3.5.1 LTE Waveform Processing . . . . . . . . . . . . . . . . . . . . . . 15

3.5.1.1 Scaling and Data Type Conversion . . . . . . . . . . . . . . . . . . . . 16

3.5.1.2 In-Situ LTE Captures, Single-UE . . . . . . . . . . . . . . . . . . . . . . . . . . 17

3.5.1.3 In-Situ LTE Captures, Multi-UE _. . . . . . . . . . . . . . . . . . . . . . . . . 18

3.5.1.4 Anechoic Chamber LTE Captures . . . . . . . . . . . . . . . . . . . . . . . . . 18

3.5.1.5 Frequency Offset Experiment Noise Extension . . . . . . . . . . . . . . . . . . . 18

3.5.2 Shaped Gaussian Noise Waveforms . . . . . . . . . . . . . . . . . . . 19

3.5.3 Summary of ABE waveforms . . . . . . . . . . . . . . . . . . . . 21

3.6 Test Automation . . . . . . . . . . . . . . . . . . . . . . . . 28 


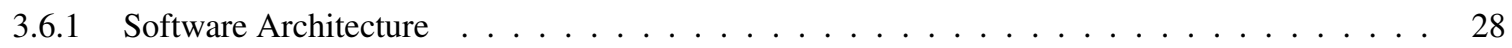

3.7 Test Process . . . . . . . . . . . . . . . . . . . . . . . . . . 30

3.7 .1 Run Organization . . . . . . . . . . . . . . . . . . . . . . . . 30

3.7.1.1 Main Experiment . . . . . . . . . . . . . . . . . . 30

3.7.1.2 Frequency Offset Experiment ...................... 31

3.7 .2 Test Execution Process . . . . . . . . . . . . . . . . . . . . . . . 32

3.7 .3 Testbed data output . . . . . . . . . . . . . . . . . . . . . . 34

4 Data Analysis Methods 3

4.1 Bit Error Rate (BER) Analysis . . . . . . . . . . . . . . . . . . . . . . . 36

4.1 .1 Point and Interval Estimation . . . . . . . . . . . . . . . . . . . . . . 36

4.1.2 Nonparametric Regression . . . . . . . . . . . . . . . . . . . . . . 36

4.1 .3 Plot Examples . . . . . . . . . . . . . . . . . . . . . . . . 37

4.2 Equivalent Impact from White Ambient Noise . . . . . . . . . . . . . . . . . . . . . . . . 40

4.2 .1 Definition of Effective Noise Level _. . . . . . . . . . . . . . . . . . . 40

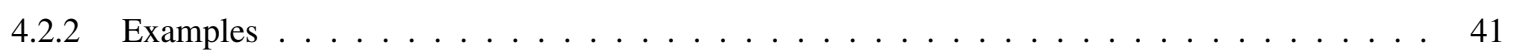

4.2.2.1 Excess Noise Tests . . . . . . . . . . . . . . . . . . . . . 41

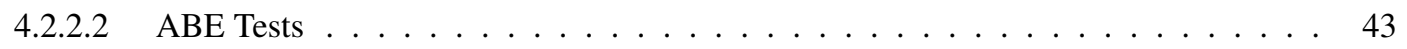

5 Primary Data Analysis Results 44

5.1 Main Experiment . . . . . . . . . . . . . . . . . . . . . . . . . . 44

5.1 ABE Power level table . . . . . . . . . . . . . . . . . . . . . . . . . . . . . . . . . . . . . . .

5.1 .2 ABE Comparison Plots . . . . . . . . . . . . . . . . . . . . . . . . . 44

5.1 .3 RX Power Threshold Plots . . . . . . . . . . . . . . . . . . . . . . . . . . 49

5.1 .4 No-ABE Comparison plots . . . . . . . . . . . . . . . . . . . . 50

5.2 Frequency Offset Experiment . . . . . . . . . . . . . . . . . . . . . 51

5.2.1 ABE Power level table . . . . . . . . . . . . . . . . . . . . . . 51

5.2 .2 Frequency Offset Results . . . . . . . . . . . . . . . . . . . . . 51

5.3 Discussion of Notable and Anomalous Results . . . . . . . . . . . . . . . . . . . . . 56

5.3.1 Unexpected Receiver Response . . . . . . . . . . . . . . . . . . . . . . . 56

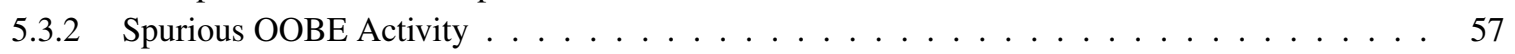

5.3 .3 ABE Waveform Processing . . . . . . . . . . . . . . . . . . . . 57

6 Secondary Data Analysis Results 59

6.1 Main Experiment: Additional Configurations . . . . . . . . . . . . . . . . . . . . . 59

6.2 Side-Experiment A: Equalizer Test . . . . . . . . . . . . . . . . . . . 63

6.3 Side-Experiment B: Space-Time Coding . . . . . . . . . . . . . . . . . . 65

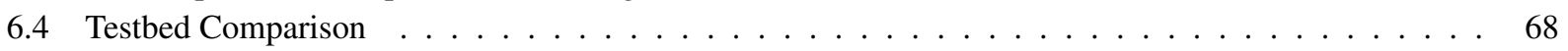

6.4.1 Examples of Consistent Test Results . . . . . . . . . . . . . . . . . . . . . 68

6.4 Examples of Inconsistent Test Results . . . . . . . . . . . . . . . . . . . . . . . . 69

7 Conclusions $\quad 70$

A Measurement Uncertainty $\mathbf{7 2}$

A.1 AMT transmitter signal power at the AMT receiver input . . . . . . . . . . . . . . 72

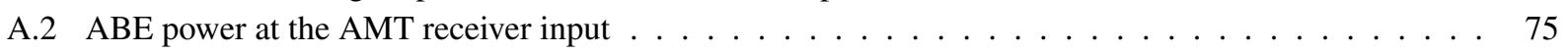

A.3 Noise at the AMT receiver input . . . . . . . . . . . . . . . . . . . . . . 79

A.4 Uncertainty for ratio quantities . . . . . . . . . . . . . . . . . . . 83

A.4.1 Eb/No ............................... 83 


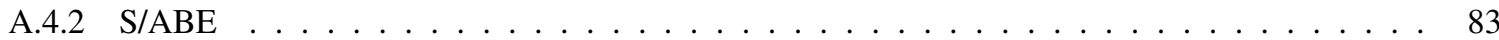

A.5 Summary of Uncertainty Values . . . . . . . . . . . . . . . . . . . . . 83

B Individual Test Plots for Main Experiment 84

C Results for Side Experiment C: Excess Noise Study 112

D Receiver-Reported Eb/No Scatterplots 115

E BER vs Eb/No in the Baseline no-ABE Case 120

F $\quad$ AMT Receiver Noise Figure 125

G Test Run Parameters and Key Performance Indicators 126

Bibliography 130 


\section{Acronyms}

\begin{tabular}{|c|c|c|c|}
\hline 3GPP & 3rd-generation partnership project & & Communications Test Network \\
\hline $\mathbf{A B E}$ & adjacent band emissions & NBIT & NIST Broadband Interoperability Testbed \\
\hline AMT & aeronautical mobile telemetry & NF & noise figure \\
\hline AGC & automatic gain control & NIST & National Institute of Standards and \\
\hline ARTM CP & M Advanced Range Telemetry continuous & & Technology \\
\hline & phase modulation & NTIA & National Telecommunications and \\
\hline AWGN & additive white Gaussian noise & & Information Administration \\
\hline AWS-1 & advanced wireless services 1 & OBW & occupied bandwidth \\
\hline AWS-3 & advanced wireless services 3 & OOB & out of band \\
\hline BEP & bit error probability & OOBE & out of band emissions \\
\hline BER & bit error rate & OQPSK & offset quadrature-phase shift keying \\
\hline CDF & cumulative distribution function & PCM/FM & pulse code modulation/frequency \\
\hline COTS & commercial off-the-shelf & & modulation \\
\hline dBFS & $\mathrm{dB}$ relative to full scale & PNG & portable network graphics \\
\hline DoD & Department of Defense & QPSK & quadrature-phase shift keying \\
\hline DQM & data quality metric & RB & resource block \\
\hline DSP & digital signal processing & RF & radio frequency \\
\hline DUT & device under test & RMS & root mean square \\
\hline EAFB & Edwards Air Force Base & $\mathbf{R X}$ & receiver \\
\hline eNB & evolved node B & SABE & signal to adjacent band emitter power ratio \\
\hline FCC & Federal Communications Commission & & \\
\hline FEC & forward error correction & SNR & signal to noise ratio \\
\hline FIR & finite impulse response & SOQPSK & shaped offset quadrature-phase shift keying \\
\hline IF & intermediate frequency & SOQPSK- & FEC SOQPSK-forward error correction \\
\hline IQ & in-phase and quadrature & STC & space-time coding \\
\hline IRIG & Inter-Range Instrumentation Group & STD & standard deviation \\
\hline ITS & Institute for Telecommunication Sciences & TAF & test automation framework \\
\hline KPI & key performance indicator & TCF & Test Configuration File \\
\hline LARC & Langley Research Center & TPF & Test Parameter File \\
\hline LDPC & low-density parity-check & TTI & transmission time interval \\
\hline LOESS & locally estimated scatterplot smoothing & TX & transmitter \\
\hline LTE & long-term evolution & UE & user equipment \\
\hline Msps & mega samples per second & UL & uplink band \\
\hline NASA & National Aeronautics and Space & VSA & vector signal analyzer \\
\hline & Administation & VSG & vector signal generator \\
\hline NASCTN & National Advanced Spectrum and & VST & vector signal transceiver \\
\hline
\end{tabular}




\section{List of Figures}

3.1 Test circuit for the susceptibility study. Two circuits are deployed, one at MITRE in Bedford, MA and one at NASCTN in Boulder, CO . . . . . . . . . . . . . . . . . . . . . . . . . . . 12

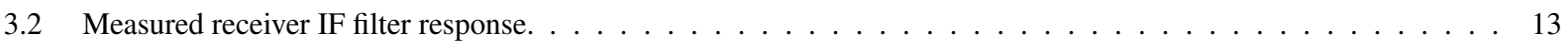

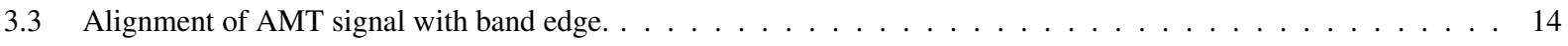

3.4 Measurement of $99 \%$ occupied bandwidth of SOQPSK 5 Mbps AMT signal. . . . . . . . . . . . . . . . . . 15

3.5 Sample of back-off frequency calculations using the spreadsheet provided by IRIG telemetry standards group. . . 16

3.6 Comparison of LTE single-UE full and $92 \mathrm{RB}$ allocation waveforms. . . . . . . . . . . . . . . . . . . . 17

3.7 Comparison of LTE single-UE unfiltered and filtered waveforms. . . . . . . . . . . . . . . . . . . . . 18

3.8 AMT and ABE frequency separation for main experiment and frequency offset experiment. . . . . . . . . . . 19

3.9 Process for extending noise floor of ABE waveforms. . . . . . . . . . . . . . . . . . . . 20

3.10 AMT and ABE frequency separation for frequency offset experiment with extended noise ABE waveform. . . . . 21

3.11 Comparison of shaped Gaussian noise spectrum. . . . . . . . . . . . . . . . . . . . . . 22

3.12 Test automation framework architecture and top-level process flow . . . . . . . . . . . . . . . . . . 29

3.13 Main experiment test execution process flow. . . . . . . . . . . . . . . . . 33

4.1 Example plot of BER vs AMT RX Power for a single whole-plot test configuration measured in Bedford. The gray boxes are $90 \%$ confidence regions for each measurement. Note that $\nu$ on the $y$-axis denotes test cases with zero observed bit errors.

4.2 Example ABE comparison plot: BER vs RX Power. The region BER $<10^{-7}$ is shaded to indicate high relative uncertainty. Note that $\nu$ on the $y$-axis denotes test cases with zero observed bit errors. . . . . . . . . . . 39

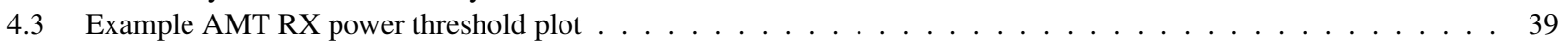

4.4 Notional plot illustrating quantities used to calculate $N_{a}^{\text {eff }} \ldots \ldots \ldots \ldots \ldots$

4.5 Example $N_{a}$ effective plot for an excess noise test . . . . . . . . . . . . . . . . . . . . . . 42

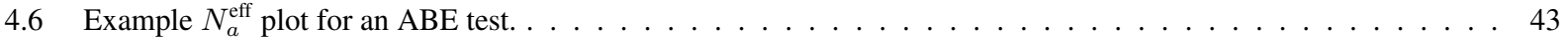

5.1 Main Experiment: ABE Comparison: PCM/FM 1 Mbps . . . . . . . . . . . . . . . . . . . . . 45

5.2 Main Experiment: ABE Comparison: PCM/FM 5 Mbps . . . . . . . . . . . . . . . . . . . . . . 46

5.3 Main Experiment: ABE Comparison: SOQPSK 5 Mbps . . . . . . . . . . . . . . . . . . . . . . 46

5.4 Main Experiment: ABE Comparison: SOQPSK $10 \mathrm{Mbps} \ldots \ldots \ldots \ldots$

5.5 Main Experiment: ABE Comparison: SOQPSK-FEC 5 Mbps . . . . . . . . . . . . . . . . . . . . . . . 47

5.6 Main Experiment: ABE Comparison: SOQPSK-FEC 10 Mbps . . . . . . . . . . . . . . . . . . . 48

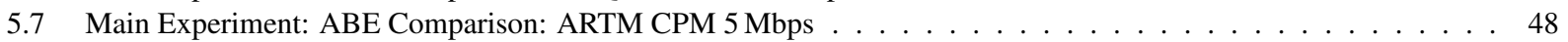

5.8 AMT RX BER $10^{-5}$ threshold scatter plots . . . . . . . . . . . . . . . . . . . . . . 49

5.9 Main Experiment: No ABE Comparison . . . . . . . . . . . . . . . . . . . . . 50

5.10 Frequency Offset Experiment: ABE Comparison: PCM/FM 1 Mbps . . . . . . . . . . . . . . . . . . 52

5.11 Frequency Offset Experiment: ABE Comparison: PCM/FM 5 Mbps . . . . . . . . . . . . . . . . . 53

5.12 Frequency Offset Experiment: ABE Comparison: SOQPSK 5 Mbps . . . . . . . . . . . . . . . . . . 53

5.13 Frequency Offset Experiment: ABE Comparison: SOQPSK 10 Mbps . . . . . . . . . . . . . . . 54

5.14 Frequency Offset Experiment: ABE Comparison: SOQPSK-FEC 5 Mbps . . . . . . . . . . . . . . . . . 54

5.15 Frequency Offset Experiment: ABE Comparison: SOQPSK-FEC $10 \mathrm{Mbps} \ldots \ldots \ldots$

5.16 Frequency Offset Experiment: ABE Comparison: ARTM CPM 5 Mbps . . . . . . . . . . . . . . . . . . . . 55

5.17 Anomalous Results investigation for PCM/FM 1 Mbps . . . . . . . . . . . . . . . . . . 56

5.18 Anomalous Results Investigation: Boulder Testbed. . . . . . . . . . . . . . . . . . . . . . . 57

5.19 Persistence spectrum and LTE timing grid overlay for the in-situ Multi-UE Az-198 ${ }^{\circ}$ (EAFB, $37 \mathrm{MHz}$ Filter). (Different color scales are used for the persistent spectrum on the left and the time segment on the right to improve visualization of the OOBEs) $\ldots \ldots \ldots \ldots \ldots \ldots \ldots$

5.20 Persistence spectrum and LTE timing grid overlay for the in-situ Multi-UE Az-76 ${ }^{\circ}$ (EAFB, $37 \mathrm{MHz}$ Filter). (Different color scales are used for the persistent spectrum on the left and the time segment on the right to improve visualization of the OOBEs $\ldots \ldots \ldots \ldots \ldots$

6.1 Main Experiment: ABE Comparison: PCM/FM 10 Mbps . . . . . . . . . . . . . . . . . . . . . . 59

6.2 Main Experiment: ABE Comparison: SOQPSK 1 Mbps . . . . . . . . . . . . . . . . . . . . . . 60

6.3 Main Experiment: ABE Comparison: SOQPSK 20 Mbps $\ldots \ldots \ldots$. . . . . . . . . . . . . 60 
6.4 Main Experiment: ABE Comparison: SOQPSK-FEC 1 Mbps . . . . . . . . . . . . . . . . . . . . 61

6.5 Main Experiment: ABE Comparison: SOQPSK-FEC 20 Mbps . . . . . . . . . . . . . . . . . . . . . 61

6.6 Main Experiment: ABE Comparison: ARTM CPM $10 \mathrm{Mbps} \ldots \ldots \ldots$

6.7 Main Experiment: ABE Comparison: ARTM CPM $20 \mathrm{Mbps} \ldots \ldots \ldots$

6.8 Side Experiment A: Equalizer/ABE comparison/Equalizer On . . . . . . . . . . . . . . . . . . . . 63

6.9 Side Experiment A: Equalizer/ABE comparison/Equalizer Off . . . . . . . . . . . . . . . . . . . . . . 64

6.10 Side Experiment B: ABE Comparison: STC 5 Mbps . . . . . . . . . . . . . . . . . . . . . . . . . 65

6.11 Side Experiment B: ABE Comparison: STC $10 \mathrm{Mbps} \ldots \ldots \ldots 6$

6.12 Side Experiment B: ABE Comparison: STC 20 Mbps . . . . . . . . . . . . . . . . . . . . . . 66

6.13 Side Experiment B: ABE Comparison: STC-FEC 5 Mbps . . . . . . . . . . . . . . . . . . . 67

6.14 Side Experiment B: ABE Comparison: STC-FEC $10 \mathrm{Mbps} \ldots \ldots \ldots$. . . . . . . . . . . . . . 67

6.15 Testbed Comparison: Examples of consistent results . . . . . . . . . . . . . . . . . . . . . 68

6.16 Testbed Comparison: Examples of inconsistent results . . . . . . . . . . . . . . . . . . . . . . . . . 69

B.1 Main Experiment configurations of pulse code modulation/frequency modulation (PCM/FM) 1 Mbps with No $\mathrm{ABE}$ and additive white Gaussian noise (AWGN) ABE. . . . . . . . . . . . . . . . . . . . . . . . . .

B.2 Main Experiment configurations of PCM/FM 1 Mbps with single-UE and multi-UE LTE ABE. . . . . . . . . . . 85

B.3 Main Experiment configurations of PCM/FM 5 Mbps with No ABE and AWGN ABE. . . . . . . . . . . . . . . 86

B.4 Main Experiment configurations of PCM/FM 5 Mbps with single-UE and multi-UE LTE ABE. . . . . . . . . . . 87

B.5 Main Experiment configurations of PCM/FM 10 Mbps with No ABE and AWGN ABE. . . . . . . . . . . . . . . 88

B.6 Main Experiment configurations of PCM/FM 10 Mbps with single-UE and multi-UE LTE ABE. . . . . . . . . . . 89

B.7 Main Experiment configurations of SOQPSK 1 Mbps with No ABE and AWGN ABE. . . . . . . . . . . . . . . . 90

B.8 Main Experiment configurations of SOQPSK 1 Mbps with single-UE and multi-UE LTE ABE. . . . . . . . . . . 91

B.9 Main Experiment configurations of SOQPSK 5 Mbps with No ABE and AWGN ABE. . . . . . . . . . . . . . . . 92

B.10 Main Experiment configurations of SOQPSK 5 Mbps with single-UE and multi-UE LTE ABE. . . . . . . . . . 93

B.11 Main Experiment configurations of SOQPSK 10 Mbps with No ABE and AWGN ABE. . . . . . . . . . . . . . . 94

B.12 Main Experiment configurations of SOQPSK $10 \mathrm{Mbps}$ with single-UE and multi-UE LTE ABE. . . . . . . . . . 95

B.13 Main Experiment configurations of SOQPSK 20 Mbps with No ABE and AWGN ABE. . . . . . . . . . . . . . . 96

B.14 Main Experiment configurations of SOQPSK $20 \mathrm{Mbps}$ with single-UE and multi-UE LTE ABE. . . . . . . . . . . 97

B.15 Main Experiment configurations of SOQPSK-forward error correction (SOQPSK-FEC) 1 Mbps with No ABE and

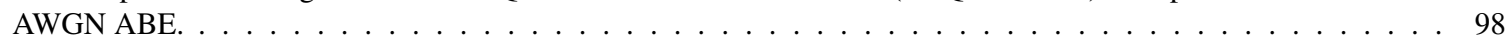

B.16 Main Experiment configurations of SOQPSK-FEC 1 Mbps with single-UE and multi-UE LTE ABE. . . . . . . . . 99

B.17 Main Experiment configurations of SOQPSK-FEC 5 Mbps with No ABE and AWGN ABE. . . . . . . . . . . . 100

B.18 Main Experiment configurations of SOQPSK-FEC 5 Mbps with single-UE and multi-UE LTE ABE. . . . . . . . . 101

B.19 Main Experiment configurations of SOQPSK-FEC 10 Mbps with No ABE and AWGN ABE. . . . . . . . . . . . 102

B.20 Main Experiment configurations of SOQPSK-FEC $10 \mathrm{Mbps}$ with single-UE and multi-UE LTE ABE. . . . . . . . 103

B.21 Main Experiment configurations of SOQPSK-FEC $20 \mathrm{Mbps}$ with No ABE and AWGN ABE. . . . . . . . . . . . 104

B.22 Main Experiment configurations of SOQPSK-FEC $20 \mathrm{Mbps}$ with single-UE and multi-UE LTE ABE. . . . . . . . 105

B.23 Main Experiment configurations of ARTM CPM 5 Mbps with No ABE and AWGN ABE. . . . . . . . . . . 106

B.24 Main Experiment configurations of ARTM CPM 5 Mbps with single-UE and multi-UE LTE ABE. . . . . . . . . . 107

B.25 Main Experiment configurations of ARTM CPM $10 \mathrm{Mbps}$ with No ABE and AWGN ABE. . . . . . . . . . . 108

B.26 Main Experiment configurations of ARTM CPM 10 Mbps with single-UE and multi-UE LTE ABE. . . . . . . . . 109

B.27 Main Experiment configurations of ARTM CPM 20 Mbps with No ABE and AWGN ABE. . . . . . . . . . . . 110

B.28 Main Experiment configurations of ARTM CPM 20 Mbps with single-UE and multi-UE LTE ABE. . . . . . . . . 111

C.1 Side Experiment C: Noise Comparison: PCM/FM 1 Mbps . . . . . . . . . . . . . . . . . . . . . . . . 112

C.2 Side Experiment C: Noise Comparison: PCM/FM 5 Mbps . . . . . . . . . . . . . . . . . . . . . . . 113

C.3 Side Experiment C: Noise Comparison: SOQPSK 5 Mbps _ . . . . . . . . . . . . . . . . . . . . 113

C.4 Side Experiment C: Noise Comparison: SOQPSK 10 Mbps . . . . . . . . . . . . . . . . . . . . . . 114

C.5 Side Experiment C: Noise Comparison: ARTM CPM 5 Mbps . . . . . . . . . . . . . . . . . . . . . . 114

D.1 $E_{b} / N_{0}$ Scatter Plots: PCM/FM 1 Mbps $\ldots \ldots \ldots \ldots \ldots$

D.2 $E_{b} / N_{0}$ Scatter Plots: PCM/FM 5 Mbps $\ldots \ldots \ldots \ldots \ldots \ldots$

D.3 $E_{b} / N_{0}$ Scatter Plots: SOQPSK 5 Mbps $\ldots \ldots \ldots \ldots \ldots \ldots \ldots \ldots$

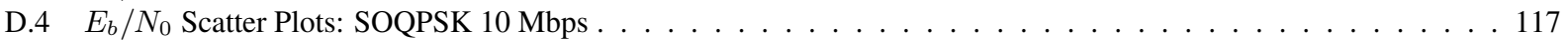

D.5 $E_{b} / N_{0}$ Scatter Plots: SOQPSK-FEC $5 \mathrm{Mbps} \ldots \ldots \ldots \ldots \ldots$

D.6 $E_{b} / N_{0}$ Scatter Plots: SOQPSK-FEC $10 \mathrm{Mbps} \ldots \ldots \ldots \ldots \ldots \ldots \ldots$

D.7 $E_{b} / N_{0}$ Scatter Plots: ARTM CPM 5 Mbps $\ldots \ldots \ldots \ldots \ldots \ldots$

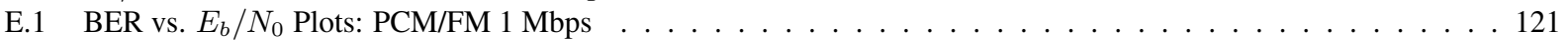




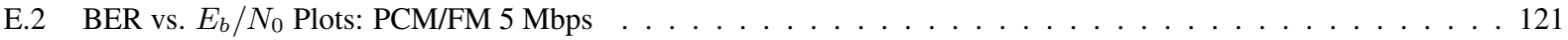

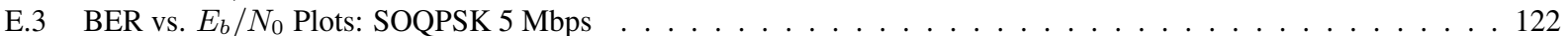

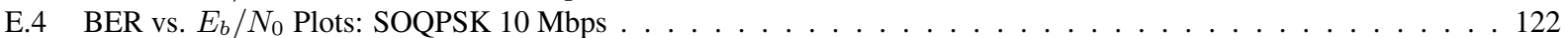

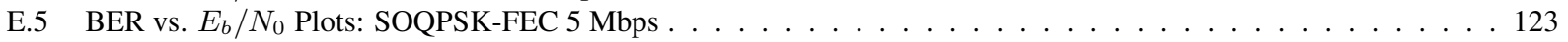

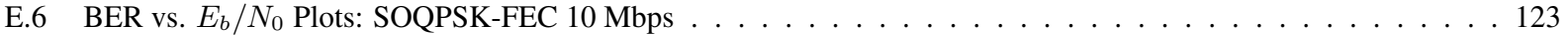

E.7 BER vs. $E_{b} / N_{0}$ Plots: ARTM CPM 5 Mbps . . . . . . . . . . . . . . . . . . . . . . . . . . . 124 


\section{List of Tables}

2.1 Factors and settings for pilot study phase 1 : baseline experiment $\ldots \ldots \ldots$

2.2 Factors and settings for pilot study phase 2 : characterization experiment . . . . . . . . . . . . 5

2.3 Factors and settings for main characterization experiment . . . . . . . . . . . . . . . . . 6

2.4 Modulation and bit rate combinations for main characterization experiment. Priority configurations shown in yellow. Only the priority configurations are tested for the frequency offset experiment. . . . . . . . . . 6

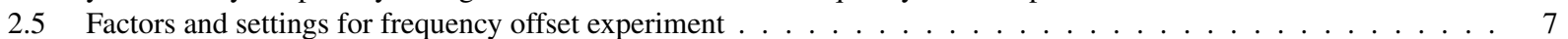

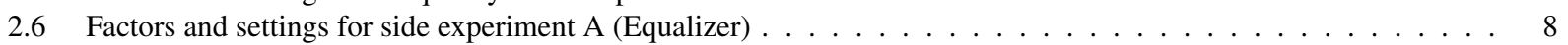

2.7 Factors and settings for side experiment B (STC) $\ldots \ldots \ldots \ldots$

2.8 Factors and settings for side experiment $\mathrm{C}$ (Excess Noise) . . . . . . . . . . . . . . . . . 8

2.9 Modulation and bit rate combinations for side experiment C. Priority configurations shown in yellow. . . . . . . 8

3.1 Test equipment used in the testbed at Bedford, MA and Boulder, CO . . . . . . . . . . . . . 23

3.2 Receiver IF filter selection for various modulations and bit rates. . . . . . . . . . . . . . . . . . . . . . . 24

$3.399 \%$ occupied bandwidth and corresponding minimum frequency for various AMT modulations and bit rates. . . 25

3.4 AMT and ABE test frequencies used in Bedford and Boulder testbeds. . . . . . . . . . . . . . . . . . . 26

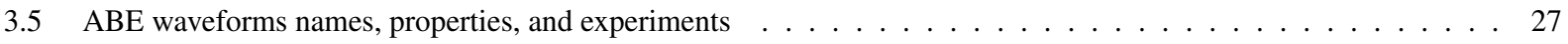

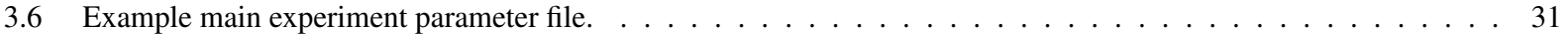

3.7 Example frequency offset experiment parameter file. . . . . . . . . . . . . . . . . . . . . 32

5.1 ABE Power level table, Main Experiment campaign conducted at the MITRE Bedford, MA testbed. . . . . . . . . 44

5.2 ABE Power level table, for the Frequency Offset Experiment as conducted at MITRE Bedford, MA . . . . . . . . 51

A.1 Power meter measurements at the face of the transmitter assembly. Note: LDPC waveforms were at code rate of $2 / 3$ and 4096 bits. . . . . . . . . . . . . . . . . . . . . . . . . . . . . . . 73

A.2 Sources of uncertainty and uncertainty budget for the AMT signal power at the plane of the receiver at the Boulder Test Site. . . . . . . . . . . . . . . . . . . . . . . . . . . 74

A.3 Sources of uncertainty and uncertainty budget for the AMT signal power at the plane of the receiver at the Bedford Test Site. . . . . . . . . . . . . . . . . . . . . . . . . . . . 74

A.4 Sources of uncertainty and uncertainty budget for the ABE power at the plane of the receiver at the Boulder Test

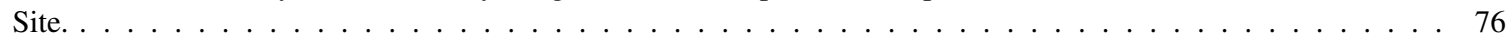

A.5 Sources of uncertainty and uncertainty budget for the ABE power at the plane of the receiver at the Bedford Test Site. . . . . . . . . . . . . . . . . . . . . . . . . . . 77

A.6 ABE Power level table, Main Experiment campaign conducted at the MITRE Bedford, MA testbed and NASCTN Boulder, CO testbed. . . . . . . . . . . . . . . . . . . . . . . . . . . . . 77

A.7 ABE Power level table, for the Frequency Offset Experiment conducted at the MITRE Bedford, MA testbed and NASCTN Boulder, CO testbed. . . . . . . . . . . . . . . . . . . . . . 78

A.8 Sources of uncertainty and uncertainty budget for applied incident noise power at the plane of the receiver at the Boulder Test Site. . . . . . . . . . . . . . . . . . . . . . . . . . . . . . . . 82

A.9 Summary of expanded uncertainty values for the various test conditions referenced to the N-type RF input connector of the AMT receiver . . . . . . . . . . . . . . . . . . . . 83

A.10 Summary of expanded uncertainty values for the various test conditions referenced to the N-type RF input connector of the AMT receiver . . . . . . . . . . . . . . . . . . . . . . 83

F.1 AMT Receiver Noise Figure . . . . . . . . . . . . . . . . . . . . . . . 125

G.1 List of Test Run parameters and AMT KPIs as reported in the test summary files . . . . . . . . . . . . 126 


\section{Chapter 1}

\section{Introduction}

The demand and evolution of frequency spectrum access requires a clear understanding of the impacts to incumbent systems as new entrants utilize the spectrum and incumbents must compress historical access requirements. Rigorous testing is a crucial element in quantifying and evaluating the potential impacts as new technologies are introduced to this increasingly congested spectrum environment. This effort, while focused on adjacent band long-term evolution (LTE) impacts on aeronautical mobile telemetry (AMT) systems, typifies the general need for rigorous evaluation of new technologies on incumbent operations.

\subsection{Objective}

The objective of this project is to design, demonstrate, and validate a test methodology to measure the impacts of LTE user equipment (UE) emissions into the adjacent AMT band. Impacts are assessed by collecting and analysing a range of performance measures, such as bit error rate (BER), received power, and $E_{b} / N_{0}$. These performance measures are generally referred to as key performance indicators (KPIs).

The validation process will include the rebroadcast and impact analysis of in-field and laboratory captures of adjacent band emissions (ABE) from LTE UEs for several AMT transmission conditions. It is important to recognize that infield measurements serve as surrogates and may not replicate the exact deployment parameters (e.g., $20 \mathrm{MHz}$ versus $10 \mathrm{MHz}$ allocations, LTE versus 5G technology) in the adjacent band. However, the in-field captures include impacts of actual telemetry receive antennas, the radio frequency (RF) connecting cables, and in-line filters. Thus, the infield captures [2] incorporate RF characteristics beyond the scope of simulation environments or typical bench-top conducted-path tests.

The analysis here focuses on BER behavior with and without the presence of ABE. A key investigation into how close the AMT frequency can be located to the band-edge of $1780 \mathrm{MHz}$ for various AMT modulation formats and bit rates, with several representative ABE. This information is helpful in establishing frequency edge back-off recommendations in the IRIG 106 Standard [1]. In addition, we explore the concept of Gaussian "equivalent noise" to aid the interpretation of the non-linear effects in AMT receiver response caused by some of the LTE waveforms.

The investigation and methodology demonstration is conducted at two testbeds, one located at the MITRE facility in Bedford, MA, and the other at the National Advanced Spectrum and Communications Test Network (NASCTN) facility in Boulder, $\mathrm{CO}$. The benefits of running multiple testbeds are the ability to validate and trouble shoot test runs, as well as increase the confidence in the reproducibility of results. A comparison between selected response curves generated by the two testbeds is found in Section 6.4.

The testbed located at the MITRE facility is considered the lead testbed as it incorporates the AMT receiver analyzer and has the most flexibility with respect to AMT modes of operation, as well as the ability to interrogate the receiver under test. The receiver analyzer is a dedicated piece of test equipment for evaluating AMT receivers. Automation code and framework development takes place on the Bedford testbed. The primary investigation data are generated on the Bedford testbed. 
The NASCTN testbed is used to prototype testing methodologies and investigate proposed testing protocols (i.e., the main and frequency offset experiments discussed below). This testbed offers greater flexibility in the monitoring and analysis of the base RF signaling conditions and also makes use of an AMT transmitter (TX) that is commonly used in telemetry operations and is able to generate most of the AMT signal conditions of interest. The investigation and concept development of Gaussian equivalent in band noise uses the NASCTN testbed, and it provides secondary investigations on several of the experiments conducted with the Bedford testbed.

In order to conserve overall testing time, the Bedford testbed is used for a side experiment focused on space-time coding (STC), while the NASCTN testbed is used for a side experiment on AMT receiver (RX) equalizer settings.

Finally, the development of automated testbeds improves the rigor of the measurement process by allowing collection of statistical rich data sets and the ability to investigate behaviors that might initially appear anomalous. Accompanying these measurements is rigorous uncertainty analysis that includes an uncertainty budget and calculation of overall measurement uncertainty. The combination of automation and remote access allows testing to continue with very minimal on-site interaction with the testbed and measurement equipment.

\subsection{Background}

In the 2010 Presidential Memorandum on Unleashing the Wireless Broadband Revolution [3], the National Telecommunications and Information Administration (NTIA) was tasked to identify underutilized spectrum suitable for wireless broadband use. In the subsequent NTIA Fast Track Report [4], many federal bands were identified as commercially viable. From this report, the Federal Communications Commission (FCC) identified $1695 \mathrm{MHz}$ to $1710 \mathrm{MHz}$, $1755 \mathrm{MHz}$ to $1780 \mathrm{MHz}$, and $2155 \mathrm{MHz}$ to $2180 \mathrm{MHz}$ together as the advanced wireless services 3 (AWS-3).

Through Auction 97 [5], the FCC auctioned the AWS-3 band for commercial mobile broadband usage in the United States. The auction raised \$41B in revenue for the United States Treasury and required federal agencies in the AWS-3 band to look for other ways to accomplish their missions. In the $1755 \mathrm{MHz}$ to $1780 \mathrm{MHz}$ portion of the AWS-3 band, the Department of Defense (DoD) is using a combination of sharing, compression, and relocation to other bands (including the $2025 \mathrm{MHz}$ to $2110 \mathrm{MHz}$ band) to adapt to the change in the spectrum allocations.

In 2017, NASCTN conducted a test campaign for Edwards Air Force Base (EAFB) measuring the out-of-band emissions of commercial LTE smartphones and an evolved node B (eNB) [6]. This report serves as a follow-on to that work in which the in-band and out-of-band LTE uplink emissions are directly injected into an AMT link in a controlled environment to evaluate the impact.

\subsection{Overview}

The collection of KPI data takes place in a laboratory environment which allows strict control over the LTE waveforms and AMT link conditions. The range of ABE waveforms includes in-situ single and multi-UE captures, Gaussian noise surrogates, and laboratory-generated, radiated LTE UE emissions. Different resource block (RB) usage allocations are tested for the single UE in-situ and laboratory-generated captures.

For this report, a subset of the collected KPIs, such as BER, are analyzed and presented. The complete set of test data for all experiments, which includes a much larger set of KPIs, is publicly available at https://doi.org/10. $18434 /$ M32279. Other analysis approaches and presentation formats of the data are possible and may provide additional insight into ABE impacts. Accompanying these measurements is a rigorous uncertainty analysis that includes an uncertainty budget and calculation of overall measurement uncertainty.

The remainder of this report is laid out as follows. Chapter 2 discusses the experiment design. Chapter 3 provides details on the testbed implementations and test execution. Chapter 4 covers data and statistical analysis methods used here. Chapter 5 presents primary data analysis results, while Chapter 6 presents some secondary analysis results. The 
report concludes with a summary of the results, a discussion on their interpretation, and ideas for future work that expands on these measurements. There are several appendices that provide additional information on measurement uncertainty (Appendix A) and additional data and analysis results (Appendix B through Appendix G). 


\section{Chapter 2}

\section{Experimental Design}

\subsection{Overview}

This chapter details the objectives, controlled factors, and settings for each experiment. The AMT receiver susceptibility testing included a total of seven different experiments: two pilot experiments, a main characterization experiment, a frequency-offset experiment, and three side experiments, labeled A, B, and C. The factors and settings for each experiment are summarized in Tables 2.1-2.9. Because changing AMT or signal generator settings required more testbed time than changing attenuation values, AMT and signal generator factors are labeled "hard-to-change" and factors depending on attenuation values are labeled "easy-to-change."

Six of the seven experiments (all except the frequency-offset experiment) include both hard-to-change and easy-tochange factors. Consequently, to efficiently utilize test time in these experiments, the number of modifications to hard-to-change factors was minimized by using a split-plot experimental design $[7,8,9]$. To execute this design, the test configurations were changed in two nested loops, where the hard-to-change factors were modified in the outer loop and the easy-to-change factors were modified in the inner loop. In the experimental design literature, blocks of hard-to-change factor configurations are called "whole-plots" and blocks of easy-to-change factors configurations are called "split-plots" [7, 8, 9].

The following sections provide specific details for each experiment.

\subsection{Pilot Study}

Prior to finalizing the main experimental design and implementation, we conducted a pilot study. The goals of the pilot study were to (i) verify the implementation of the testbed automation (across two testbeds) and (ii) obtain preliminary data to inform subsequent experimental design.

The pilot study included two experiments, a baseline experiment and a characterization experiment. The experimental factors and settings used for baseline and characterization experiments are listed in Table 2.1 and Table 2.2, respectively. Overall, there were six experimental factors for the pilot study: modulation type, bit rate, frequency offset between the AMT band and ABE allocation, ABE type, ABE level, and AMT signal level. These factors are labeled A-F, respectively. Note that factors A-D are denoted as hard-to-change and factors $\mathrm{E}$ and $\mathrm{F}$ are denoted easy-to-change.

The aim of the baseline experiment was to verify testbed repeatability, e.g., to verify that signal and ABE levels did not drift in time. For the baseline experiment, factors A through D were held constant with the settings listed in Table 2.1, and all combinations of factors $\mathrm{E}$ and $\mathrm{F}$ were tested for a total of 33 unique configurations. In other words, the baseline experiment tested a single whole-plot configuration over a split-plot configuration block. The baseline test was repeated seven times on the Bedford testbed.

The aims of the characterization experiment were to verify testbed operation and to collect preliminary data to inform subsequent experiments. As indicated in Table 2.2, there were a total of 40 whole-plot configurations of hard-to-change factors and 33 split-plot configurations of easy-to-change factors, yielding a total of 1320 unique test configurations. 
Table 2.1: Factors and settings for pilot study phase 1: baseline experiment

\begin{tabular}{|c|l|c|c|c|}
\hline Id & \multicolumn{1}{|c|}{ Factor } & Settings & \# Levels & Easy/Hard to Change \\
\hline A & Modulation Type & SOQPSK & 1 & Hard \\
\hline B & Bit Rate & $10 \mathrm{Mbps}$ & 1 & Hard \\
\hline C & Frequency Offset & $0 \mathrm{MHz}$ & 1 & Hard \\
\hline D & ABE Type & AWGN $(\mathrm{BW}=20 \mathrm{MHz})$ & 1 & Hard \\
\hline E & ABE Level & None, Mid, High & 3 & Easy \\
\hline F & AMT Signal & 1 dB steps on grid spanning a & 11 & Easy \\
\hline
\end{tabular}

Table 2.2: Factors and settings for pilot study phase 2: characterization experiment

\begin{tabular}{|c|l|c|c|c|}
\hline Id & \multicolumn{1}{|c|}{ Factor } & Settings & \# Levels & Easy/Hard to Change \\
\hline A & Modulation Type & PCMFM, SOQPSK, SOQPSK-FEC, STC, STC-FEC & 5 & Hard \\
\hline B & Bit Rate & $1,10 \mathrm{Mbps}$ & 2 & Hard \\
\hline C & Frequency Offset & $0,2.5 \mathrm{MHz}$ & 2 & Hard \\
\hline D & ABE Type & AWGN $(\mathrm{BW}=20 \mathrm{MHz})$, in situ LTE capture (single-UE Full) & 2 & Hard \\
\hline E & ABE Level & None, Mid, High & 3 & Easy \\
\hline F & $\begin{array}{l}\text { AMT Signal } \\
\text { Level }\end{array}$ & $1 \mathrm{~dB}$ steps on grid spanning a & 11 & Easy \\
\hline
\end{tabular}

Due to their preliminary nature, the results of the pilot study are not presented in this report. However, the results of the pilot study provided important insights to improve the reliability of testbed automation and for the selection of signal level ranges in the main characterization experiment, described next.

\subsection{Main Characterization Experiment}

The objective of the main characterization experiment was to assess the impact of different ABE types on AMT receiver performance. In particular, the experiment aimed to test a range of conditions including the transition from a strong link $\left(\mathrm{BER}<10^{-7}\right)$ to a poor link $\left(\mathrm{BER}>10^{-4}\right)$.

The factors and settings used for the experiment are summarized in Table 2.3. Four factors were varied: modulation type, bit rate, ABE type, and AMT signal level. The first three factors are hard-to-change (whole-plot) factors and the last factor is an easy-to-change (split-plot) factor.

One of the lessons-learned from the pilot study was that the factors could be combined and organized differently to reduce the total number of factors and better focus the experimental design on relevant test conditions. Namely, compared to the pilot study design (Table 2.2), the number of factors was reduced from six to four by making the following adjustments. First, the frequency offset factor in the pilot study was removed and instead folded into the ABE type. For example, the additive white Gaussian noise (AWGN) ABE type included three bandwidths, 20, 18, and 16.5 MHz, corresponding to three different frequency offsets, 0,2 , and $3.5 \mathrm{MHz}$, respectively, from the AMT band. Second, the ABE level factor was eliminated, the baseline no-ABE condition was instead included as an ABE type, and a single testbed attenuator setting was used for all $\mathrm{ABE}$ waveforms. 
Table 2.3: Factors and settings for main characterization experiment

\begin{tabular}{|c|l|c|c|c|}
\hline Id & \multicolumn{1}{|c|}{ Factor } & Settings & \# Levels & Easy/Hard to Change \\
\hline A & Modulation Type & PCM/FM, SOQPSK, SOQPSK-FEC, ARTM-CPM & 4 & Hard \\
\hline B & Bit Rate & $1,5,10,20$ Mbps & 4 & Hard \\
\hline C & ABE Type & $\begin{array}{c}\text { None, AWGN (BW=20, 18, 16.5 MHz), in-situ LTE } \\
\text { captures: single-UE (92 RB, Full), multi-UE (EAFB } \\
\text { Az76, EAFB Az198, Lang Az140, Lang Az165) }\end{array}$ & 10 & Hard \\
\hline D & AMT Signal Level & $\begin{array}{c}1 \text { dB steps on grid spanning a } \\
10 \mathrm{~dB} \text { range with config-dependent shift }\end{array}$ & 11 & Easy \\
\hline
\end{tabular}

Table 2.4: Modulation and bit rate combinations for main characterization experiment. Priority configurations shown in yellow. Only the priority configurations are tested for the frequency offset experiment.

\begin{tabular}{|l|l|l|l|l|}
\hline Modulation Type & \multicolumn{4}{|c|}{ Bit Rate (Mlops) } \\
\hline PCM/FM & 1 & 5 & 10 & \\
\hline SOQPSK & 1 & 5 & 10 & 20 \\
\hline SOQPSK- FEC & 1 & 5 & 10 & 20 \\
\hline ARTM CPM & & 5 & 10 & 20 \\
\hline
\end{tabular}

The fourteen combinations of modulation type and date rate listed in Table 2.4 were tested. Note that atypical configurations (pulse code modulation/frequency modulation (PCM/FM) $20 \mathrm{Mbps}$, Advanced Range Telemetry continuous phase modulation (ARTM CPM) 1 Mbps) were excluded. Further, the combinations highlighted in yellow were prioritized for testing because they are of highest interest to AMT operators.

Apart from modulation type and date rate settings, all other combinations of the settings listed in Table 2.3 were tested. Overall, there were 140 whole-plot configurations and 11 split-plot configurations, yielding a total of 1540 unique test configurations.

All configurations were tested twice on the Bedford testbed. In addition, a subset of configurations (all except with shaped offset quadrature-phase shift keying (SOQPSK)-forward error correction (FEC)), were tested twice on the Boulder testbed.

\subsection{Frequency Offset Experiment}

The aim of the frequency offset experiment was to characterize the impact on AMT receiver performance when the AMT center frequency is shifted relative to ABE. More specifically, a key goal was to assess the effectiveness of the frequency back-off distances recommended in the IRIG 106 standard [1, Sec. A.12] for operation near the telemetry band edge as a method for mitigating receiver impacts from $\mathrm{ABE}$.

The factors and settings for the frequency offset experiment are listed in Table 2.5. There are four factors: modulation type, bit rate, ABE type, and frequency offset between the AMT signal and ABE. Only the seven modulation and bit rate combinations shown in yellow in Table 2.4 were tested for the frequency offset experiment, yielding a total of $7 \times 10 \times 12=840$ unique test configurations.

All configurations were tested twice on the Bedford testbed, and a subset (all except with SOQPSK-FEC) were tested 
Table 2.5: Factors and settings for frequency offset experiment

\begin{tabular}{|c|l|c|c|c|}
\hline Id & \multicolumn{1}{|c|}{ Factor } & \multicolumn{1}{c|}{ Settings } & \# Levels & Easy/Hard to Change \\
\hline A & Modulation Type & PCM/FM, SOQPSK, SOQPSK-FEC, ARTM-CPM & 4 & Hard \\
\hline B & Bit Rate & $1,5,10$ Mbps & 4 & Hard \\
\hline C & ABE Type & $\begin{array}{r}\text { AWGN 20 MHz, in-situ LTE captures: single-UE (92 RB, } \\
\text { Full, 92 RB filtered, Full filtered), multi-UE: (EAFB Az76, } \\
\text { EAFB Az198), lab LTE captures (20 MHz Full, 20 MHz } \\
\text { upper 10 RB blanked, 5 MHz Full) }\end{array}$ & 10 & Hard \\
\hline D & Frequency Offset & $\begin{array}{r}1 \mathrm{MHz} \text { steps on grid including IRIG-recommended offset } \\
1\end{array}$ & 12 & Hard \\
\hline
\end{tabular}

twice on the Boulder testbed.

\subsection{Supplemental Experiments}

To supplement the primary experiments described above, three additional side experiments were performed that include an additional receiver feature, modulation format, and potential performance evaluation method. These additional experiments address emerging aspects of AMT receiver operations and susceptibility testing. This section describes the purpose and details for each side experiment.

\subsubsection{Side Experiment A: Equalizer}

The aim of side experiment A was to evaluate the impact of enabling the equalizer on the AMT receiver performance in the presence of ABE. The factors and settings are listed in Table 2.6. Only one modulation and bit rate configuration was tested: SOQPSK at $5 \mathrm{Mbps}$, with the equalizer enabled. The remaining factor settings were the same as for the main characterization experiment. The 110 unique test configurations for this experiment were tested twice on the Boulder testbed.

\subsubsection{Side Experiment B: Space-Time Coding}

The purpose of side experiment B was to assess the effect of utilizing the STC option on the AMT system in the presence of ABE. The factors and settings for this experiment are given in Table 2.7. Specifically, the STC option was tested with and without FEC for $5 \mathrm{Mbps}$ and $10 \mathrm{Mbps}$ bit rates. The remaining factors and settings were the same as the main characterization experiment. In total, 440 unique configurations were tested twice on the Bedford testbed.

\subsubsection{Side Experiment C: Excess Noise}

The aim of side experiment $\mathrm{C}$ was to evaluate the impact of in-band excess white noise on AMT performance. Here, excess noise was injected into the testbed with a noise diode, as explained in Chapter 3.

The factors and settings for this experiment are listed in Table 2.9. Four factors were varied: modulation type, bit rate, excess noise level, and AMT signal level. Ten combinations of modulation type and bit rate were tested, as shown in Table 2.9. Also, in addition to a baseline with no excess noise, three levels of excess noise were tested, denoted Low, Mid, and High. In total, 440 unique configurations were tested twice on the Boulder testbed. 
Table 2.6: Factors and settings for side experiment A (Equalizer)

\begin{tabular}{|c|l|c|c|c|}
\hline Id & \multicolumn{1}{|c|}{ Factor } & Settings & \# Levels & Easy/Hard to Change \\
\hline A & Modulation Type & SOQPSK with equalizer enabled & 1 & Hard \\
\hline B & Bit Rate & 5 Mbps & 1 & Hard \\
\hline C & ABE Type & $\begin{array}{c}\text { None, AWGN (BW=20, 18, 16.5 MHz), in-situ LTE } \\
\text { captures: single-UE }(92 \mathrm{RB}, \text { Full), multi-UE (EAFB } \\
\text { Az76, EAFB Az198, Lang Az140, Lang Az165) }\end{array}$ & 10 & Hard \\
\hline D & AMT Signal Level & $\begin{array}{c}1 \mathrm{~dB} \text { steps on grid spanning a } \\
10 \mathrm{~dB} \text { range with config-dependent shift }\end{array}$ & 11 & Easy \\
\hline
\end{tabular}

Table 2.7: Factors and settings for side experiment B (STC)

\begin{tabular}{|c|l|c|c|c|}
\hline Id & \multicolumn{1}{|c|}{ Factor } & Settings & \# Levels & Easy/Hard to Change \\
\hline A & Modulation Type & STC, STC-FEC & 2 & Hard \\
\hline B & Bit Rate & $5,10 \mathrm{Mbps}$ & 2 & Hard \\
\hline C & ABE Type & $\begin{array}{c}\text { None, AWGN (BW=20, 18, 16.5 MHz), in-situ LTE } \\
\text { captures: single-UE }(92 \mathrm{RB}, \text { Full), multi-UE (EAFB } \\
\text { Az76, EAFB Az198, Lang Az140, Lang Az165) }\end{array}$ & 10 & Hard \\
\hline D & AMT Signal Level & $\begin{array}{c}1 \mathrm{~dB} \text { steps on grid spanning a } \\
10 \mathrm{~dB} \text { range with config-dependent shift }\end{array}$ & 11 & Easy \\
\hline
\end{tabular}

Table 2.8: Factors and settings for side experiment C (Excess Noise)

\begin{tabular}{|c|l|c|c|c|}
\hline Id & \multicolumn{1}{|c|}{ Factor } & Settings & \# Levels & Easy/Hard to Change \\
\hline A & Modulation Type & PCM/FM, SOQPSK, ARTM-CPM & 3 & Hard \\
\hline B & Bit Rate & $1,5,10,20$ Mbps & 4 & Hard \\
\hline C & Excess Noise Level & None, Low, Mid, High & 4 & Easy \\
\hline D & AMT Signal Level & $\begin{array}{c}1 \mathrm{~dB} \text { steps on grid spanning a } \\
10 \mathrm{~dB} \text { range with config-dependent shift }\end{array}$ & 11 & Easy \\
\hline
\end{tabular}

Table 2.9: Modulation and bit rate combinations for side experiment C. Priority configurations shown in yellow.

\begin{tabular}{|l|l|l|l|l|}
\hline Modulation Type & \multicolumn{4}{|c|}{ Bit Rate (Mbps) } \\
\hline PCM/FM & 1 & 5 & 10 & \\
\hline SOQPSK & 1 & 5 & 10 & 20 \\
\hline ARTM CPM & & 5 & 10 & 20 \\
\hline
\end{tabular}




\subsection{Implementation Details}

\subsubsection{Randomization}

To alleviate the influence of uncontrolled factors, sound experimental practice dictates that the test order of experimental configurations should be randomized [8,9]. Indeed, our intention was to randomize the order of tests to the extent possible. However, due to implementation complexities and post-hoc adjustments that were necessary for some ABE waveforms, the test order was not randomized in an ideal manner.

Specifically, in the split-plot experiments, the order of the split-plot configurations was randomized, but the order of the whole-plot configurations was not randomized, The frequency offset experiment, which only included hard-tochange factors, randomized the test order for one factor (frequency offset), but did not randomize over the remaining factors. In addition, for all $\mathrm{ABE}$ experiments, due to modifications to some $\mathrm{ABE}$ waveforms as well as adjustments to the range of AMT signal levels, a subset of configurations was retested at the end of the experiment.

Although we cannot rule out a bias in the results due to incomplete test randomization, any such bias is likely to be small for the following reasons. First, as noted in Chapter 3, the test equipment was reset each time a new whole-plot configuration was loaded onto the testbed, which should mitigate potential hysteresis (memory) effects of the testbed. Second, although the whole-plot configuration order was not randomized, repetitions of each configuration were tested on different days at different times. Third, as shown in Chapter 6, repeated tests indicated strong reproducibility across the two testbeds, which used different test equipment.

\subsubsection{ABE Waveform Selection and Conditioning}

The in-situ LTE waveform captures from two geographically disparate sites [2] present a large raw data set of UE uplink band (UL) activity in the advanced wireless services 1 (AWS-1) band for potential playback in the testbeds. Here the AWS- 1 collection is a stand-in for probable user activity in the AWS-3 band. The waveforms collection is categorized: first, a single UE, where NASCTN researchers collect radiation from a test UE in near proximity to the AMT asset, and second, the emissions of the LTE UL allocation inherently observable by the AMT asset (that contain multiple UE emissions).

Of the single UE waveforms, we select a scenario with an $10 \mathrm{Mbps}$ upload rate, a very large bit rate that exercises the $100 \mathrm{RB}$ allocation bandwidth. The high bit rate is necessary to ensure persistent observable activity in the upper RBs, as these RBs represent the smallest frequency separation to the AMT band of interest.

Down selected waveforms of the multi-UE category exhibit a combination of consistency in RB activity over all available resource blocks within an approximately $500 \mathrm{~ms}$ playback time window and greater than $20 \mathrm{~dB}$ in LTE power per RB above the noise floor in the recordings. The selections for the measurement campaign here exhibit waveform properties such as over all RB activity and power levels, that were likely to cause impact on the AMT receiver.

The LTE ABE waveforms captured in the controlled laboratory anechoic environment are of a single UE in the AWS-3 band. This methodology for the laboratory acquisition is outlined in an accompanying document [10]. Here the singleUE in-situ test scenarios informed the laboratory controlled LTE network parameters, RB activity, and link budgets. In addition, waveforms with RB blanking settings, which is a feature not typically employed in live networks but touted to be an efficient method to mitigate adjacent band interference by not scheduling or using RBs while transmitting, are exercised in the testbeds.

The ABE Gaussian noise waveforms are generated with software over bandwidths of $16.5,18$, and $20 \mathrm{MHz}$ for comparison purposes. These bandwidths and center frequencies of $1770 \mathrm{MHz}$ (for $18 \mathrm{MHz}$ and $20 \mathrm{MHz}$ cases) and 1769.25 MHz (for the 16.5 MHz case) are chosen for comparison to LTE scenarios (See figure 3.11). The $20 \mathrm{MHz}$ case covers the upper $20 \mathrm{MHz}$ portion of the $1755 \mathrm{MHz}-1780 \mathrm{MHz}$ AWS-3 Band. This represents LTE activity closest 
to the lower edge of the AMT band but not necessarily representative of actual LTE implementations. The $18 \mathrm{MHz}$ represents the occupied bandwidth of fully utilized RBs within a $20 \mathrm{MHz}$ LTE allocation. 3rd-generation partnership project (3GPP) defines a one $\mathrm{MHz}$ guard band on either side of a $20 \mathrm{MHz}$ allocation. Finally, the $16.5 \mathrm{MHz}$ case is same as $18 \mathrm{MHz}$ case but leaves the equivalent of approximately eight RBs unused at the upper portion of the LTE band along with the one $\mathrm{MHz}$ guard band requirement.

After down selection, the ABE waveforms are filtered in with either a $20 \mathrm{MHz}$ or $37 \mathrm{MHz}$ filter to replicate the fundamental LTE (with its inherent guard band) portion of the waveform, or the LTE with captured out of band emissions (OOBE) that extends beyond the $20 \mathrm{MHz}$ LTE allocation, respectively. The first case is useful to investigate the impacts of the fundamental LTE allocation and is intended to mimic software-simulated LTE or laboratory-emulated LTE equipment (e.g., a vector signal analyzer (VSA) with an LTE emulation package). In these cases, real-world OOBE is not part of the waveform. The $37 \mathrm{MHz}$ filtering includes both the allocated LTE and the OOBE beyond the fundamental LTE allocation. Testing with this case helps determine how the overall in-situ LTE waveforms impact the AMT receivers. The main experiment utilizes the $20 \mathrm{MHz}$ filtering and the frequency offset experiments use both the $20 \mathrm{MHz}$ and the $37 \mathrm{MHz}$ filtering. Section 3.5 provides additional details on processing specifics and ABE waveforms. 


\section{Chapter 3}

\section{Test Implementation and Execution}

\subsection{Overview}

Testbed design and test execution are inherently linked. Testbed features determine the translation from theoretical design of experiments to practical testing implementation. This chapter describes the testbed design, including the RF circuity, instrumentation, and automation framework. In addition, the details on the execution processes and configurations for the tests in Chapter 2 are provided.

\subsection{Testbed Design}

The design of the testbed is derived to achieve multiple objectives. First, the testbed must support a high degree of automation, requiring the incorporation of instruments and devices that may not have a well-functioning machine interface. Second, the testbed needs to cover a wide range of test conditions in RF signaling, AMT signal scenarios, and $\mathrm{ABE}$ conditions. Third, the testbed ought to be implemented with easily reproducible components, for the most part commercial off-the-shelf (COTS) hardware to aid in making the test campaign approachable in replication.

The test RF circuit diagram is shown in Figure 3.1. This circuit allows for combining and level setting several RF sources, while taking into account measurement and RF test circuit design best practices with regards to isolation, added RF noise, shielding, and adequate number of test ports for calibration investigations. Table 3.1 list the devices used in the two testbeds constructed for the testing done here. The number of particular devices used, such as the number of programmable attentuators, is not shown.

The testbed allows for combining three signal sources: the AMT signal, the ABE waveforms, and calibrated RF thermal noise from a noise diode. The combination of relative signal strengths at the plane of the receiver are achieved through varying settable inline attenuators controlled by the automation software. Automation of the testbeds, including the software architecture and interfacing to COTS equipment, is discussed in Section 3.6.

\subsection{AMT Signals}

The two testbeds use two different transmitters: the Boulder testbed has a $5 \mathrm{~W}$ output power, field-deployable AMT transmitter that could be used in a DoD test article, while the Bedford testbed transmitter uses a receiver analyzer that contains an AMT transmitter as well as other supporting hardware such as a noise source. In each testbed, the transmitters function as AMT signal generators to create an AMT link to the AMT receiver. The testbeds use AMT receivers, of the same model and firmware, that would be deployed at a DoD test range for a closer representation to fielded systems and due to the wide variety of KPI available on the receivers. Both sets of AMT hardware comply with the DoD telemetry standards [1].

In addition, parameters such as the receiver intermediate frequency (IF) filter response and distance from the frequency band edge (i.e., distance from the ABE) are important considerations in the test setup. Details on how those parameters are determined for the testing are discussed in Sections 3.3.1 through 3.3.3. 


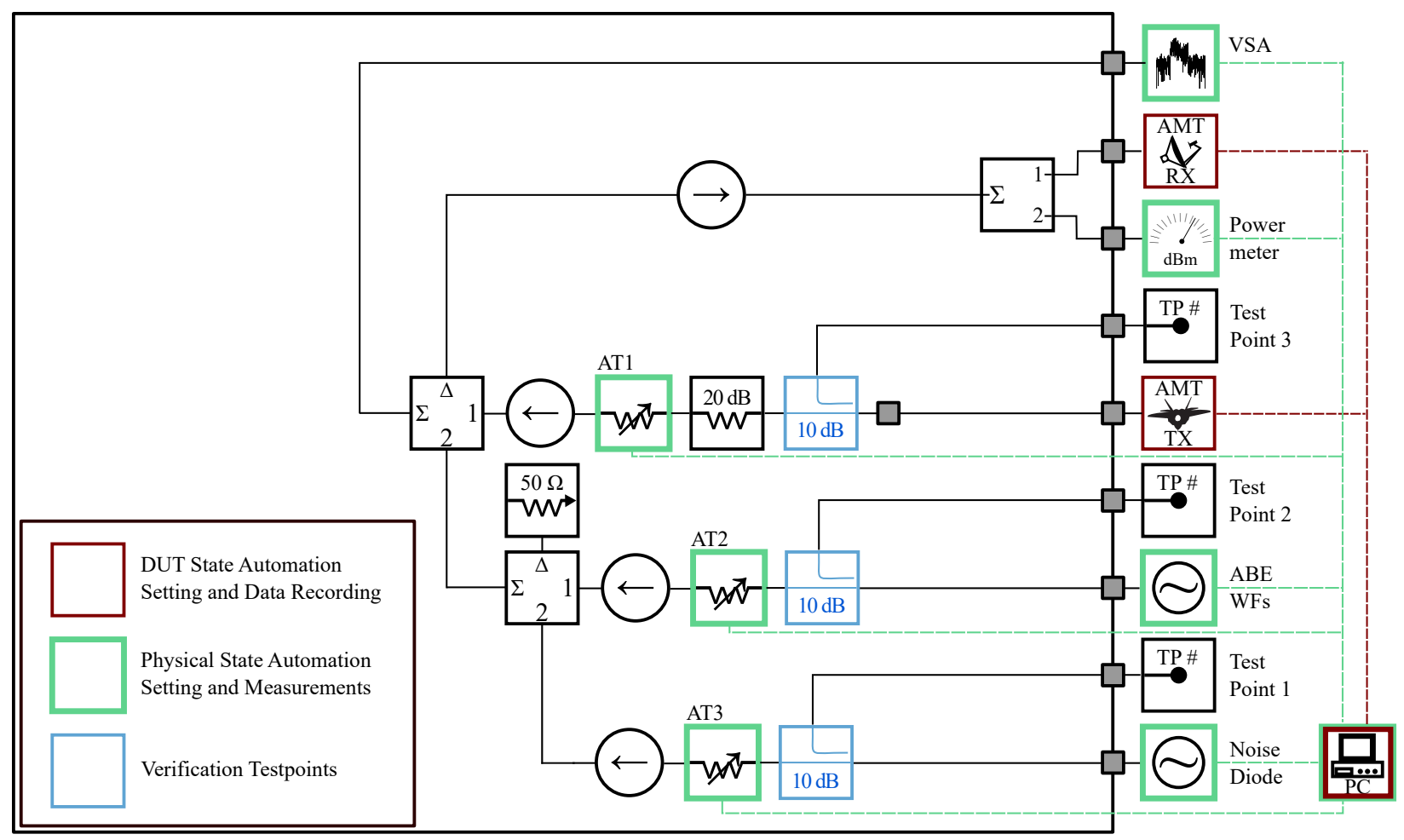

Figure 3.1: Test circuit for the susceptibility study. Two circuits are deployed, one at MITRE in Bedford, MA and one at NASCTN in Boulder, CO.

\subsubsection{Receiver IF Filter Response}

The AMT receivers under test have a selectable IF filter bank that is automatically set based on the receiver's modulation, coding and bit rate configuration. The IF filters have nominal bandwidths ranging from $70 \mathrm{kHz}$ to $40 \mathrm{MHz}$. Table 3.2 lists the IF filters that are automatically selected for each combination of modulation, coding and bit rate used in this study.

The IF filter, which largely dictates the selectivity of the receiver, has a significant impact on the performance of the receiver in the presence of ABE. For this study it is important to examine the frequency response of the filters to help understand the receiver performance and explain test results. Therefore the frequency responses of all IF filters listed in Table 3.2 are measured.

The filters are measured by connecting a wideband noise source to the RF input of the receiver and examining the receiver's IF output spectrum on a Keysight MXA-Series spectrum analyzer. The analyzer measures the absolute power of the IF output, (which is a function of the input signal level and receiver gain), but for the purpose of filter characterization, the relative power is appropriate. Therefore the spectrum analyzer traces are post-processed to normalize the responses. As a benchmark the $3 \mathrm{~dB}$ bandwidths are calculated as well. The results are shown in Figure 3.2. Note that during these measurements the receiver's automatic gain control (AGC) function remains active. As the bandwidth of the IF filter increases, the in-band power increases as well because more of the wideband noise in the receiver signal path is allowed to pass through the filter. The AGC responds by reducing its gain. When the spectrum analyzer traces are normalized as shown in Figure 3.2, this gives the false impression of an increase in the measurement noise floor. 


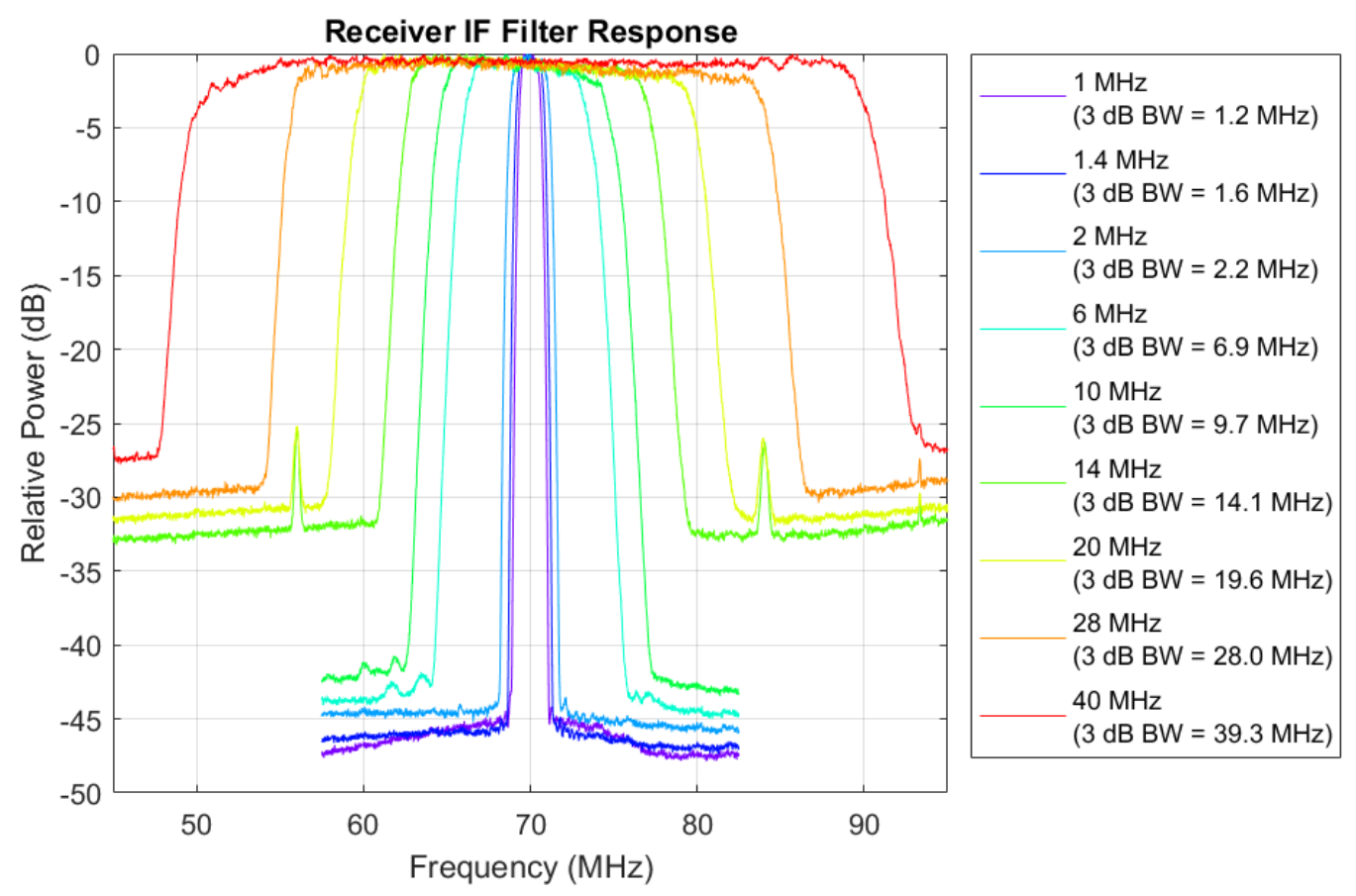

Figure 3.2: Measured receiver IF filter response.

\subsubsection{Center Frequency Selection}

DoD test ranges that support missions requiring aeronautical telemetry frequently use the "upper" $\mathrm{L}$ band. After the FCC Auction 97, the upper L band now occupies $1780 \mathrm{MHz}$ to $1850 \mathrm{MHz}$ [11]. Thus, the $1780 \mathrm{MHz}$ frequency is now a border between commercial mobile broadband in the AWS-3 band (currently LTE UL) and DoD mobile allocations. If an AMT link operates using frequencies close to the LTE UL band, there is a risk that the UL power could enter the AMT receiver as an unwanted signal and adversely affect AMT receiver performance. The adjacent band signals injected into the NASCTN testbeds occupy spectrum up to $1780 \mathrm{MHz}$ (including the inherent guard band when testing LTE uplinks). Depending on the testing scenario, the AMT signals' center frequencies were either held at a fixed distance from the band edge (determined by AMT transmit power, modulation formats, and bit rates) or stepped over a range of center frequencies.

\subsubsection{AMT Occupied Bandwidth and Minimum Test Frequency}

The center frequency of the AMT signal must also account for the AMT signal occupied bandwidth (OBW). A signal's 99\% OBW is defined as the the bandwidth containing $99 \%$ of its total transmitted power. The OBW of the AMT signal is measured and used to determine the minimum frequency at which less than $1 \%$ of the transmitted AMT signal power extends beyond the lower limit of the upper L band, and into the adjacent band AWS-3 band. This minimum AMT frequency is given by

$$
F_{A M T \mid M I N}=F_{U P P E R \_L \mid M I N}+O B W / 2
$$

where $F_{U P P E R \_L \mid M I N}$ is the lower limit of the upper L band, or $1780 \mathrm{MHz}$. Figure 3.3 illustrates the resulting alignment of the transmitted AMT signal with the band edge.

For the OBW measurements, the AMT signal is generated by the receiver analyzer listed in Table 3.1 at a center 


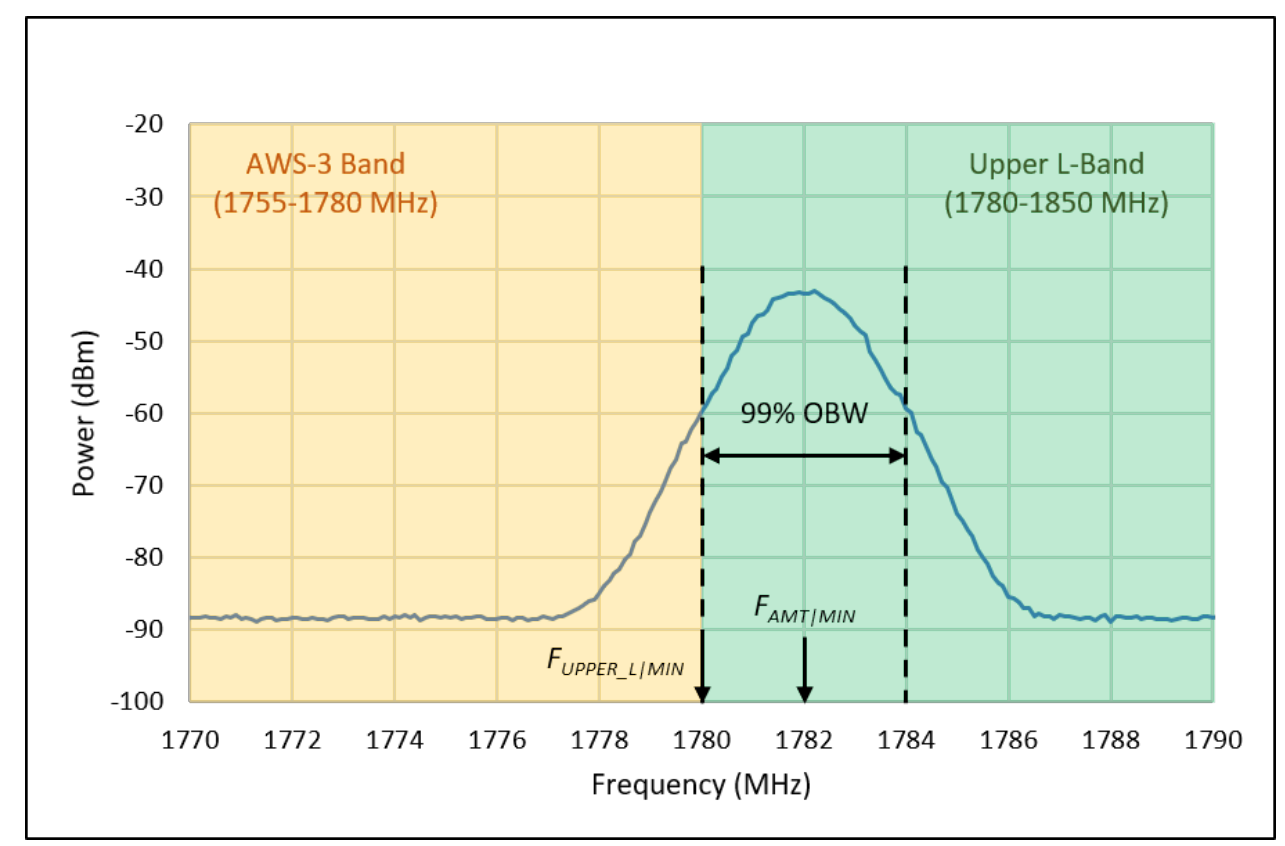

Figure 3.3: Alignment of AMT signal with band edge.

frequency of $1780 \mathrm{MHz}$. The receiver analyzer output is connected directly to the input of a Keysight MXA-Series spectrum analyzer, and the spectrum analyzer's built-in OBW measurement mode is utilized. Figure 3.4 shows an example OBW measurement for a SOQPSK 5 Mbps AMT signal. A summary of measured OBW and the corresponding minimum AMT frequencies for various modulations and bit rates is given in Table 3.3.

\subsubsection{IRIG Band Edge Backoff Frequencies}

In DoD test and training missions that require telemetry, frequency assignments are provided by a test range frequency manager to minimize interference. In the bands used for telemetry, spectrum managers implement guard bands in frequency using the frequency assignment rules defined in the Inter-Range Instrumentation Group (IRIG) standards [1]. Table A-5 in section A.12 of the IRIG provides a spreadsheet calculator to determine valid telemetry frequency assignments when operating close to the $1780 \mathrm{MHz}$ band edge. These assignments are a function of the AMT transmit power, bit rate, and modulation type. A sample screenshot of the IRIG spreadsheet calculation is shown in Figure 3.5, the values in red text show that, for example, a center frequency of $1782.5 \mathrm{MHz}$ is appropriate for PCM/FM at a power of $37 \mathrm{dBm}$, and a bit rate $1 \mathrm{Mbps}$ at the lower edge of $1780 \mathrm{MHz}-1850 \mathrm{MHz}$ band. If transmitting a SOQPSK $5 \mathrm{Mbps}$ AMT signal, a center frequency of $1784.5 \mathrm{MHz}$ is assigned. The tests here cover back-off frequency ranges that include those prescribed by the IRIG from the $1780 \mathrm{MHz}$ band-edge for a variety AMT and ABE signal combinations.

\subsection{Adjacent Band Emissions}

ABE testing is defined in the IRIG-118 Telemetry Test Standard, Volume 2, Chapter 4.22 TEST: Receiver Adjacent Channel Interference and Chapter 7.5 TEST: Demodulator Adjacent Channel Interference Test, [12]. The IRIG-118 adjacent band testing methods are to measure effects of additional AMT transmissions in spectrum above and below the intended AMT transmission. However, the methods do not include specifics for testing against LTE adjacent band transmissions or surrogate waveforms.

The IRIG-118 states "a specialized test set can replace most of this test equipment if one is available" [12], page 601. The NASCTN testbeds, which generate LTE from in-phase and quadrature (IQ) recordings and digital signal processing (DSP) synthesized Gaussian noise ABE waveforms from vector signal generator (VSG)s, are a specialized 


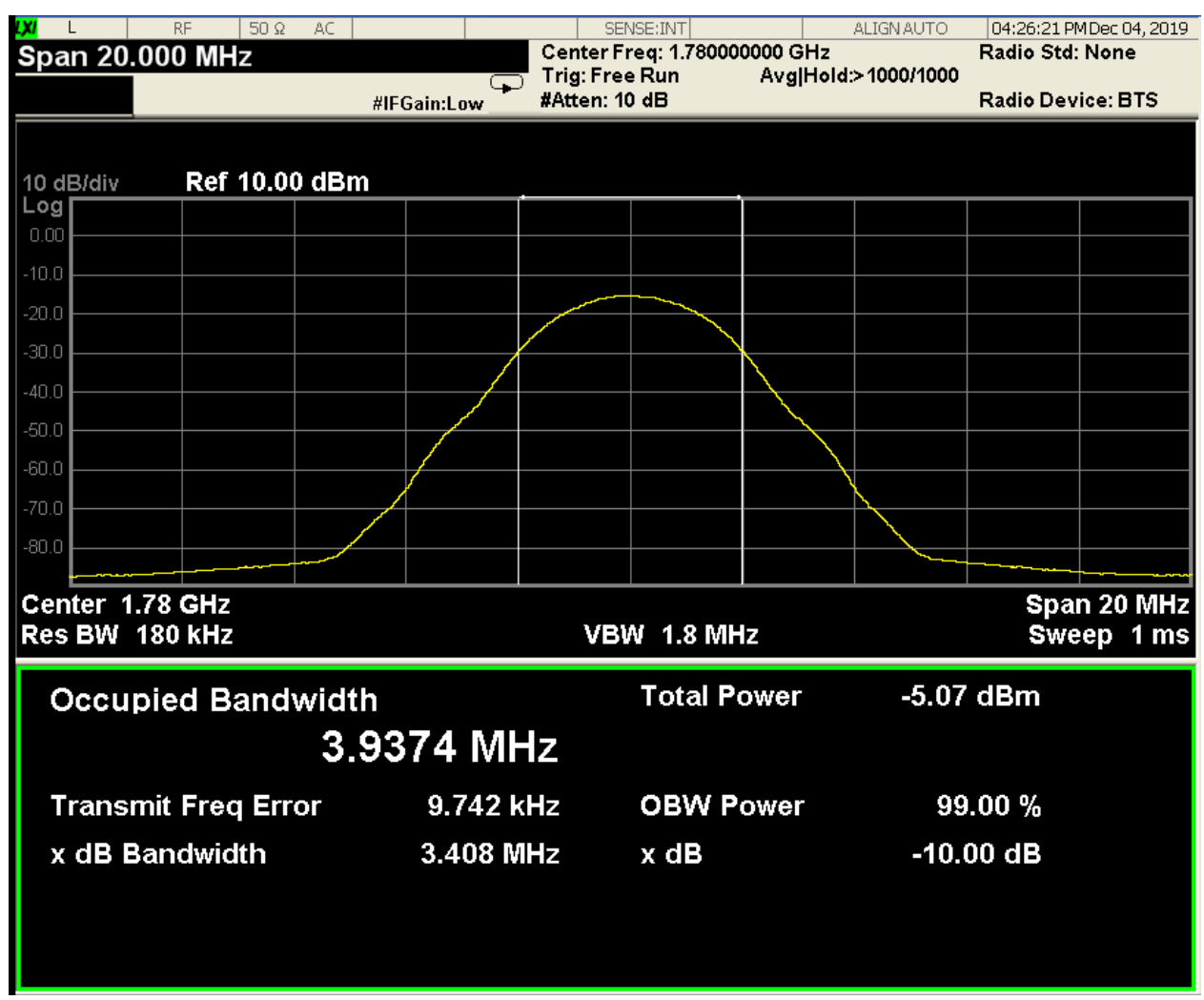

Figure 3.4: Measurement of $99 \%$ occupied bandwidth of SOQPSK 5 Mbps AMT signal.

test setup for adjacent band testing. Note that for this test campaign the NASCTN ABE waveforms require additional signal processing before playback on the testbed's vector signal transceiver (VST) as described in Section 3.5.1.

\subsection{Testbed Differences for AMT and ABE Test Frequencies}

The main experiment tests call for the AMT center frequency set as close as possible to the band edge, $F_{A M T \mid M I N}$, and the ABE center frequency set nominally to $1770 \mathrm{MHz}$. However, differences in AMT transmitters between the Bedford and Boulder testbeds leads to slight differences in AMT test frequencies. The Bedford testbed uses a receiver analyzer to generate the AMT signal. The receiver analyzer output frequency is tunable in $1 \mathrm{kHz}$ steps, so it is set to the exact frequency. However, the Boulder testbed uses an AMT TX, which is tunable in steps of $0.25 \mathrm{MHz}$, so it was set to the closest possible value within that resolution. To maintain the same separation between the AMT and ABE signals, the ABE center frequency in the Boulder testbed is offset accordingly. A summary of the AMT and ABE center frequencies used in the Bedford and Boulder testbeds is provided in Table 3.4. Note that testing using SOQPSK low-density parity-check (LDPC) and either of the STC modes is only performed in Bedford.

\subsubsection{LTE Waveform Processing}

$\mathrm{ABE}$ waveforms generated in the testbeds are one of three types:

1. software generated shaped Gaussian noise,

2. an in-situ capture of LTE uplink activity, or

3. an anechoic chamber capture of laboratory-controlled LTE uplink activity.

The LTE waveforms captured in-situ and in the anechoic chamber require various forms of processing prior to usage 


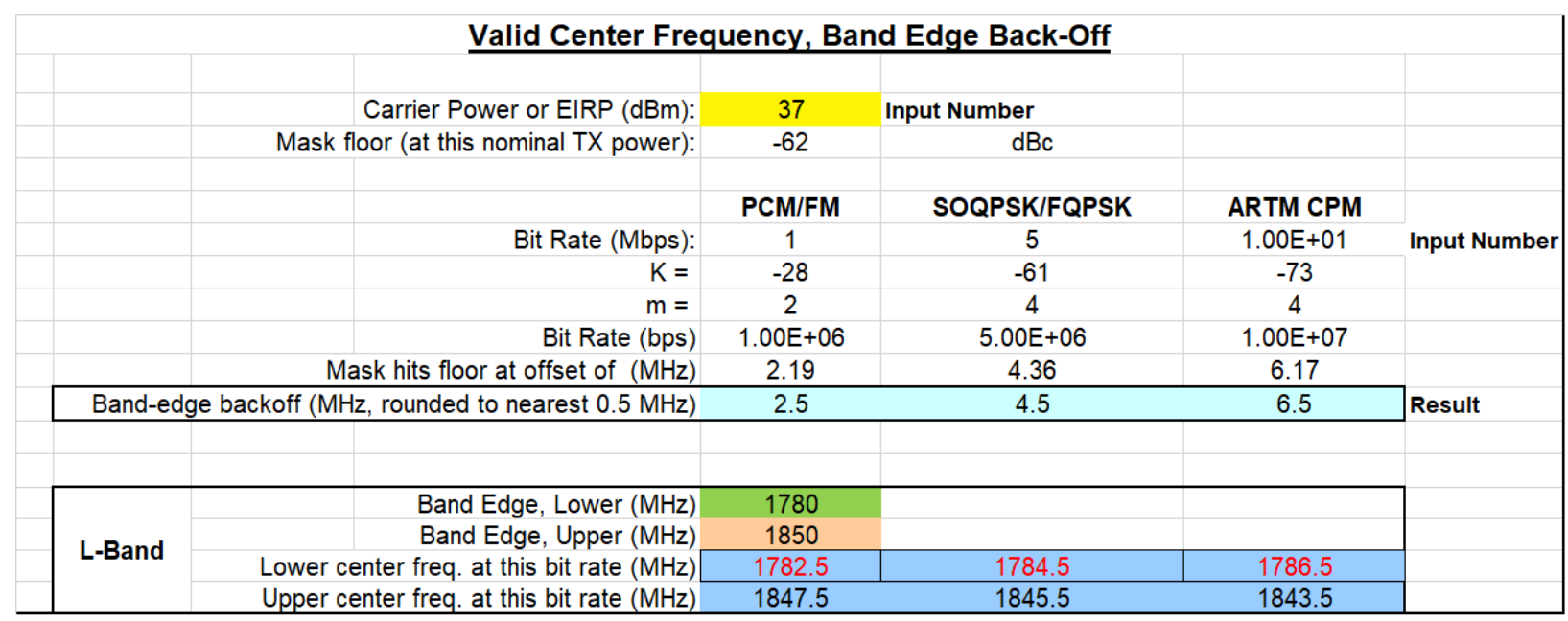

Figure 3.5: Sample of back-off frequency calculations using the spreadsheet provided by IRIG telemetry standards group.

in the testbed. This processing is necessary to optimize the VSG playback of the waveforms, and, in some cases, to achieve the waveform characteristics needed to meet the experiment objectives. The subsections 3.5.1.1 through 3.5.1.5 describe the processing performed universally on all waveforms, as well as the processing specific to the in-situ LTE captures. Additional processing needed for the frequency offset experiment is also discussed.

\subsubsection{Scaling and Data Type Conversion}

The VSGs that generate the ABE waveforms require the IQ components of the waveforms to be represented by 16-bit signed integers. The VSGs have a fullscale magnitude equal to the maximum value for a 16-bit signed integer, or 32,767 . To optimize the use of the instrument's available dynamic range, the waveforms must be scaled such that the peak magnitude of the waveform is slightly below the fullscale value. Determining the peak magnitude involves a balance of dynamic range and distortion. For this experiment a peak magnitude of $-6 \mathrm{dBFS}$ is chosen to achieve low distortion while maintaining sufficient dynamic range.

The VSAs that capture the LTE signals save IQ waveform data to files. However the range of values in these waveforms is dependent on the input signal level and instrument settings such as the reference level, and are not optimized for the VSG. The raw capture data can also be in various formats. Therefore, in preparation for use in the testbed, all waveforms are scaled and converted into binary files as required by the VSGs.

The target peak magnitude for the waveforms is given by

$$
V_{\text {peak }}=V_{F S} \times 10^{\left(\frac{A}{20}\right)}
$$

where $V_{F S}$ is the fullscale magnitude of the instrument, or 32,767 , and $A$ is the target peak magnitude relative to fullscale in logarithmic terms, or $-6 \mathrm{dBFS}$. The waveform is then scaled by

$$
X_{\text {scaled }}=V_{\text {peak }} \times\left(\frac{X}{\max (|X|)}\right)
$$

where $X$ is the raw complex waveform. Note that $|X|$ in Equation 3.3 returns the complex magnitude. 


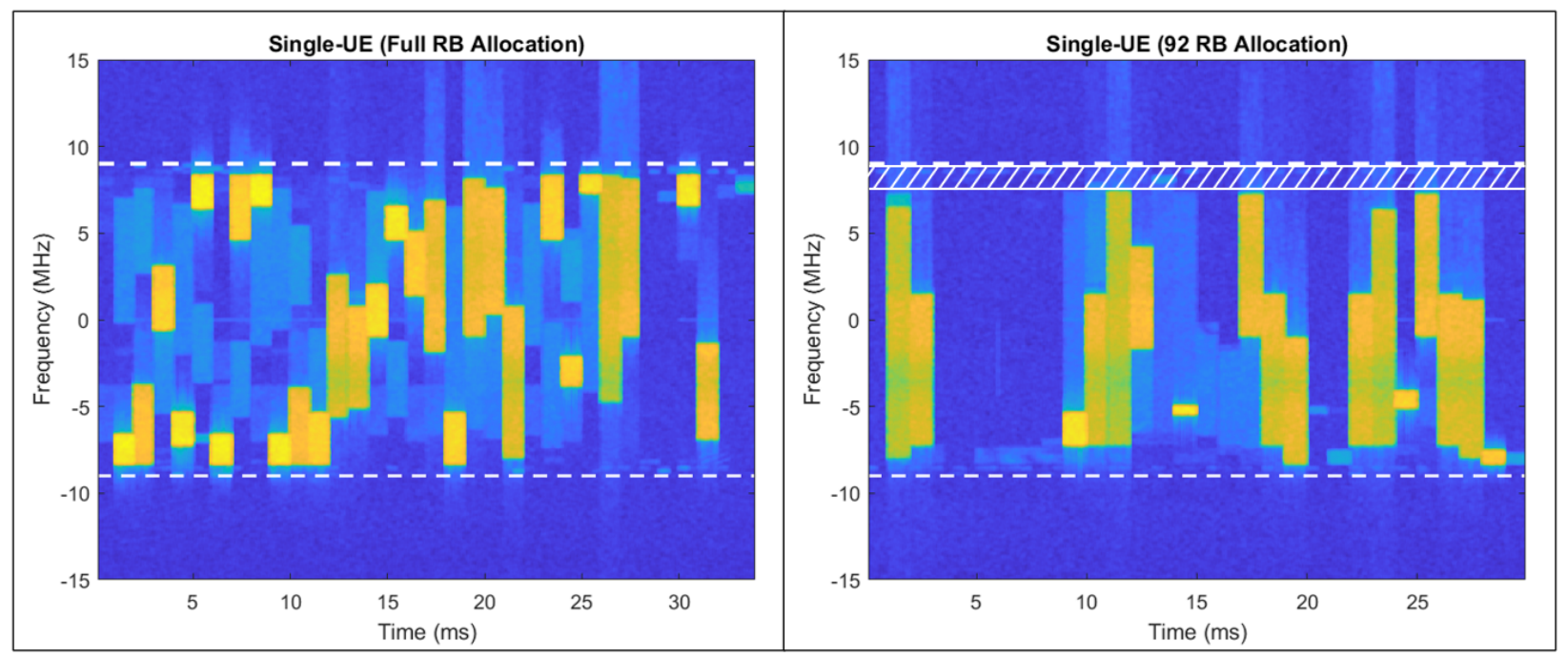

Figure 3.6: Comparison of LTE single-UE full and 92 RB allocation waveforms.

Finally $X_{\text {scaled }}$ is rounded to the nearest integer, and the waveform values are converted to 16-bit signed integers and saved to a binary file. The waveform in this state is compatible with the National Instruments PXIe-5673E VSG used in the Bedford testbed. However, an additional conversion step is needed for compatibility with the Rhode \& Schwarz SMW200A VSG used in the Boulder testbed. Rhode \& Schwarz "ARB Toolbox" software is used to convert the binary file to the file format needed by the instrument. Also, due to differences in how the the output power is set on the two VSGs, care is taken to ensure that the output power of the two instruments was consistent.

\subsubsection{In-Situ LTE Captures, Single-UE}

The in-situ LTE single-UE capture obtained at EAFB is approximately $5 \mathrm{~s}$ in length. The experimental design calls for two subsets of this waveform, one with LTE uplink activity in all RBs and another with uplink activity in only the lower 92 RBs. To identify appropriate waveform segments, spectrograms of the entire waveform are computed and the RB allocation for each subframe is cataloged. From this RB allocation catalog, waveform segments are identified that meet the criteria. Test efforts target an interval within the single-UE uplink recording that did not utilize the upper RB regions while maintaining the same uplink data rate. This subset is selected to investigate the possibility for additional frequency separation by means other than frequency shifting.

Figure 3.6 shows spectrograms of the resulting waveform segments. The white dashed lines mark the frequency limits of the resource grid at $\pm 9 \mathrm{MHz}$. Note that an LTE waveform with a nominal bandwidth of $20 \mathrm{MHz}$ utilizes an actual bandwidth of $18 \mathrm{MHz}$, with a guardband of $1 \mathrm{MHz}$ at either end. The figure on the left shows the Full RB Allocation waveform segment, which has activity spanning nearly the full range of RBs. The figure on the right shows the $92 R B$ Allocation waveform segment, with activity spanning only the lower $92 \mathrm{RBs}$. In this figure, the white hatched lines mark the region of the resource grid corresponding with the upper 8 RBs. An LTE RB is $180 \mathrm{kHz}$ wide, so this 8 RB region encompasses the upper $1.44 \mathrm{MHz}$. The waveform segments are also chosen such that they begin and end on empty subframes to minimize the impact of any phase discontinuity in the transition from the end of one one cycle to the beginning of the next cycle. During playback these waveforms repeat every $30 \mathrm{~ms}$ and $34 \mathrm{~ms}$ for the Single UE 92 RB and Single UE Full RB allocation, respectively.

Finally, as discussed in Section 2.6.2, the in-situ LTE captures are filtered to isolate the fundamental LTE portion of the waveform. A bandpass finite impulse response (FIR) filter with a passband of $\pm 9 \mathrm{MHz}$ and stopband of $\pm 10 \mathrm{MHz}$ is applied to the waveforms. Figure 3.7 shows the persistence spectra for the waveform before and after the filter is 


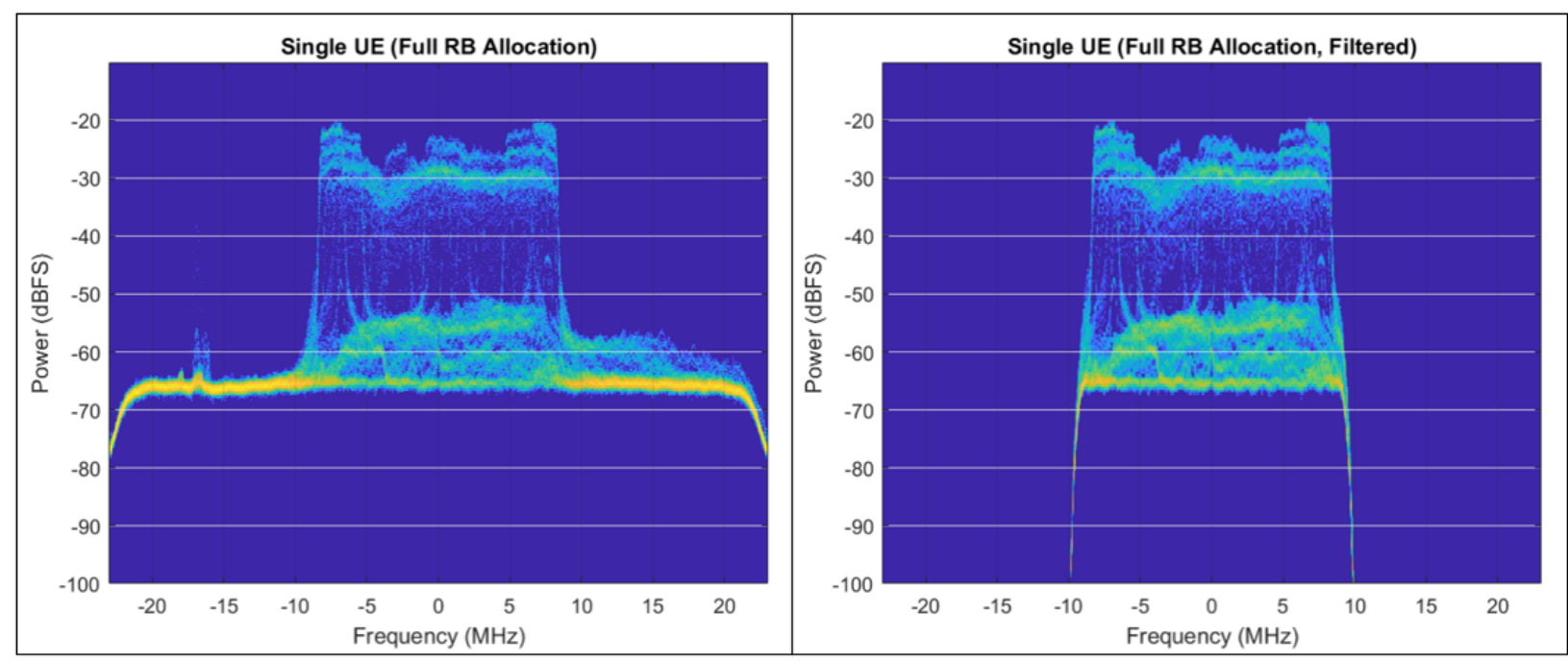

Figure 3.7: Comparison of LTE single-UE unfiltered and filtered waveforms.

applied.

\subsubsection{In-Situ LTE Captures, Multi-UE}

In-situ multi-UE captures are processed in largely the same way as the in-situ single-UE captures. The only difference is that the waveform segment is longer and is selected without targeting any particular RB allocation. The raw captures are either $2 \mathrm{~s}$ or $5 \mathrm{~s}$ in length. Shorter testbed waveform segments for playback are extracted from these raw captures. The waveform segments begin approximately $5 \mathrm{~ms}$ into the raw capture and have a duration of $500 \mathrm{~ms}$. The exact times are chosen such that the waveform segments begin and end on empty subframes. As with the other waveforms, the in-situ multi-UE waveforms are scaled and converted for optimization and compatibility with the VSGs.

\subsubsection{Anechoic Chamber LTE Captures}

As with the in-situ multi-UE captures, the processing performed on the anechoic chamber captures is extraction of $500 \mathrm{~ms}$ waveform segments and scaling for optimization with the VSGs.

\subsubsection{Frequency Offset Experiment Noise Extension}

For all main experiment tests, the AMT frequency is set such that the $99 \%$ occupied bandwidth of the AMT signal aligns with the lower edge of upper $\mathrm{L}$ band as shown in Figure 3.3. For these tests, the nominal ABE bandwidth (set by the bandwidth of the waveform capture equipment) spans the the entire AMT receiver passband. The impact of any in-band component of the ABE waveform on receiver performance can be examined. For example, this AMT in-band component can be the wideband noise floor of an antenna feed network or may include emissions in the band adjacent to the LTE band at the time of waveform capture.

For the frequency offset experiment the AMT frequency is varied throughout the test run. As the separation between the AMT and ABE signal increases, the ABE bandwidth no longer spans the entire AMT receiver passband. When the ABE band edge falls within the receiver's passband, this creates an invalid test condition that is illustrative more of test equipment limitations than actual emissions in the AMT environment. This condition is illustrated in Figure 3.8. The figure on the left is an example of a main experiment test. The shaded region represents the approximate passband of the AMT receiver, which typically extends slightly into the AWS-3 band as noted by the dashed line. Due to the minimal frequency separation the in-band component of the $\mathrm{ABE}$ waveform, which in this case is the noise floor of the AMT antenna feed network, extends through the entire passband of the AMT receiver. This in-band component of $\mathrm{ABE}$ is well defined and its impact on receiver performance can be examined. The figure on the right represents 


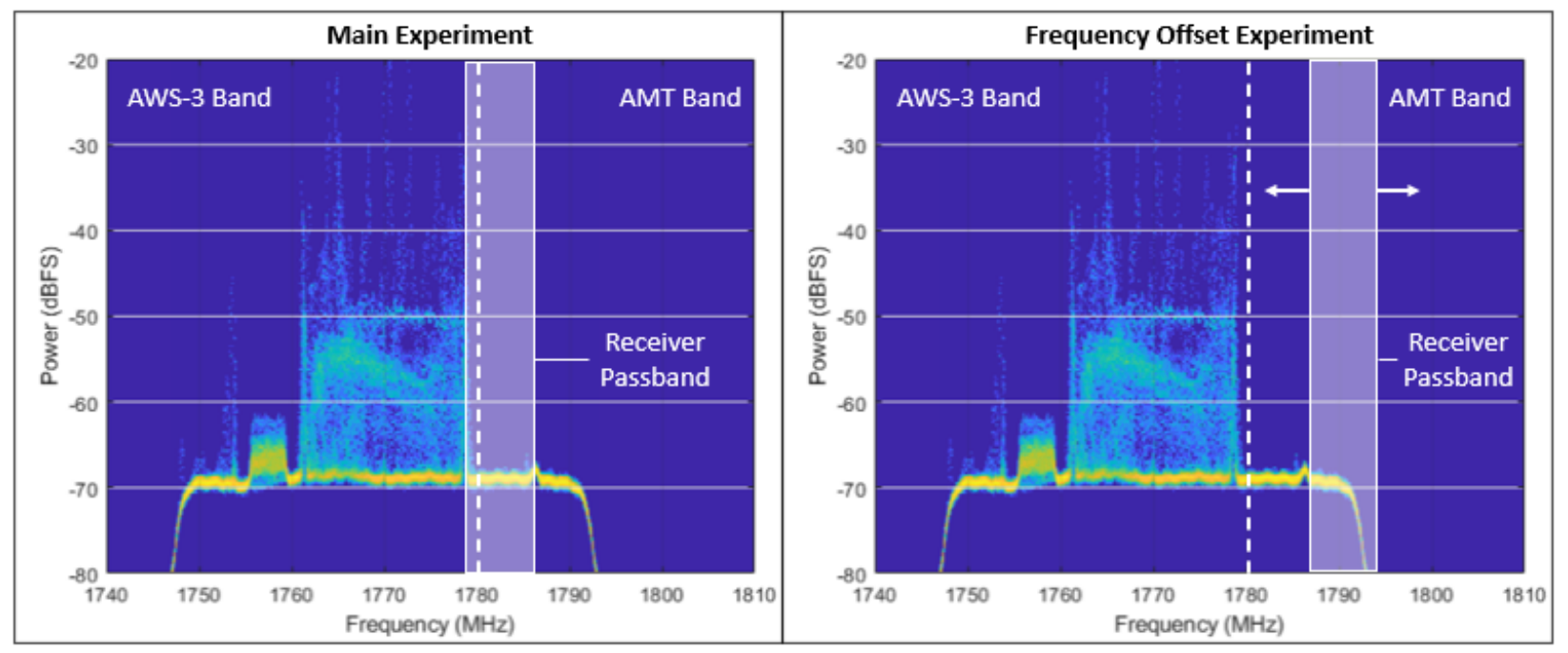

Figure 3.8: AMT and ABE frequency separation for main experiment and frequency offset experiment.

a frequency offset experiment test, where the AMT frequency varies throughout the test run. In this case the in-band component of $\mathrm{ABE}$ changes with frequency separation, which is not representative of the actual AMT environment.

To address this, the ABE waveforms for the frequency offset experiment are modified to extend the noise floor, such that a constant noise floor exists for all frequency separations. The process for extending the noise floor is illustrated in Figure 3.9. Figure 3.9(A) shows the persistence spectrum for the original ABE waveform. The maximum bandwidth of this waveform is limited by the sample rate that the waveform was captured at 46.08 mega samples per second (Msps). In order to extend the noise floor, the sample rate first needs to be increased. Figure 3.9(B) shows the waveform after it has been resampled by a ratio of $13 / 6$, resulting in a new sample rate of 99.84 Msps. At this point the spectral characteristics of the waveform have not changed, it's just sampled at a higher rate to allow higher frequency components to be added without aliasing. Next, the waveform is filtered with either a $37 \mathrm{MHz}$ bandpass filter (Figure 3.9(C)) or $20 \mathrm{MHz}$ bandpass filter (Figure 3.9(D)). The filter selection depends on the objectives of the experiment, as discussed in Section 2.6.2. If the objective is to evaluate the impact of the just the fundamental LTE signal, and exclude any emissions adjacent to the LTE signal that might have been captured, then the $20 \mathrm{MHz}$ filter is applied. However, if the objective is to include emissions adjacent to the LTE band, then the $37 \mathrm{MHz}$ filter is applied. Outside of a bandwidth of approximately $37 \mathrm{MHz}$, the performance of the VSA used to capture the waveforms is not considered a calibrated capture, and thus is not used. Next, a shaped Gaussian noise waveform is created that matches the ABE noise floor and complements the filter cutoff. Figure 3.9(E) shows an example of a shaped noise waveform for the $37 \mathrm{MHz}$ filtered waveform. Finally the filtered waveform and shaped noise are combined, resulting in an ABE waveform with extended noise as shown in Figure 3.9(F).

Figure 3.10 shows the $\mathrm{ABE}$ waveform and receiver passband, represented by the shaded region, for an example frequency experiment test using an extended noise $\mathrm{ABE}$ waveform. The figure illustrates that the $\mathrm{ABE}$ noise floor remains constant in the receiver passband for any frequency offset.

\subsubsection{Shaped Gaussian Noise Waveforms}

As discussed in Section 2.6.2, shaped Gaussian noise waveforms with bandwidths of $20 \mathrm{MHz}, 18 \mathrm{MHz}$ and $16.5 \mathrm{MHz}$ are created to compare receiver performance with the LTE ABE waveforms. To create these shaped waveforms, a wideband complex noise waveform is first generated that consists of uniquely random real and imaginary time series each with a normal distribution. This non-shaped noise waveform is scaled to a peak magnitude $-6 \mathrm{dBFS}$. Finally, 


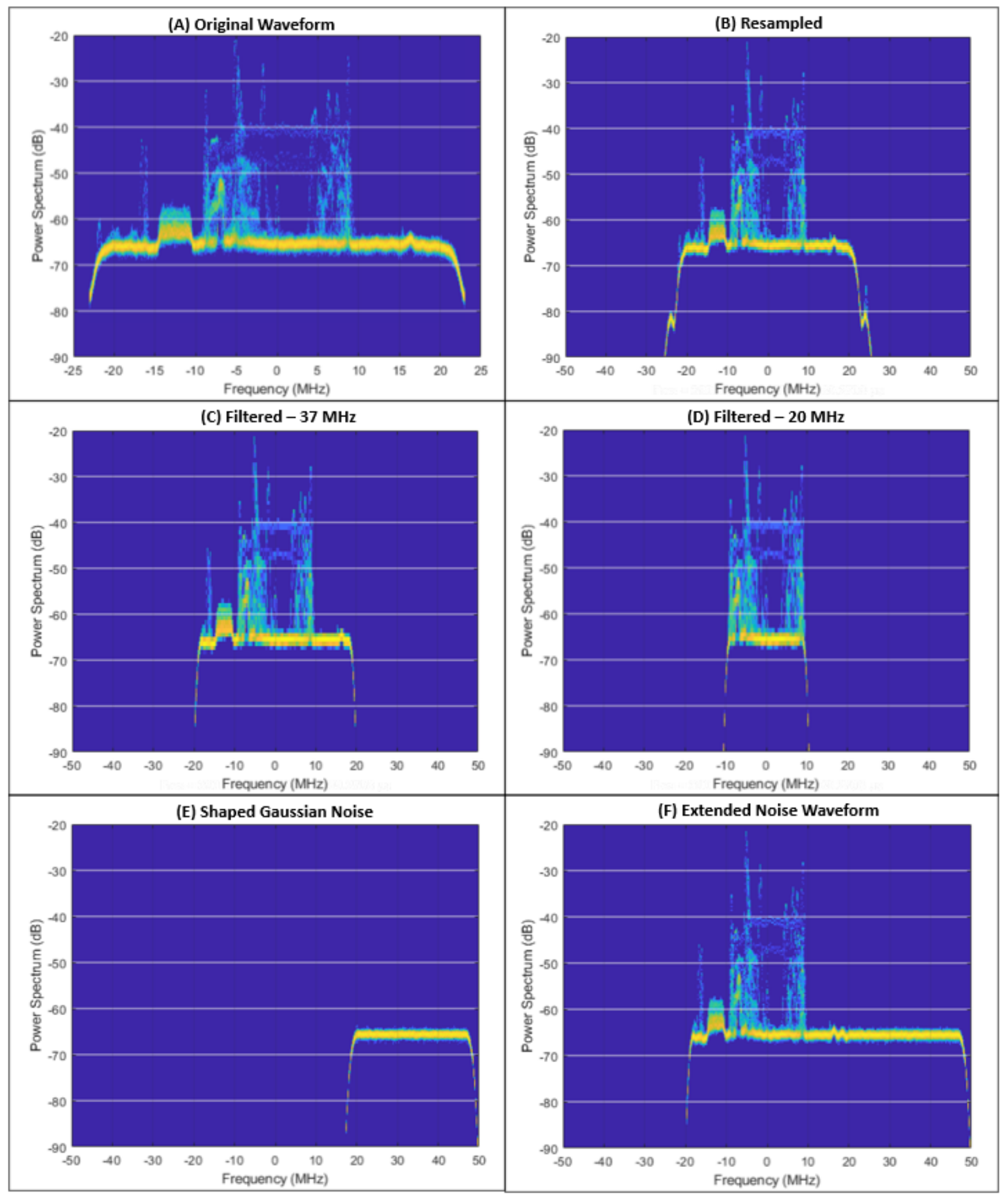

Figure 3.9: Process for extending noise floor of $\mathrm{ABE}$ waveforms. 


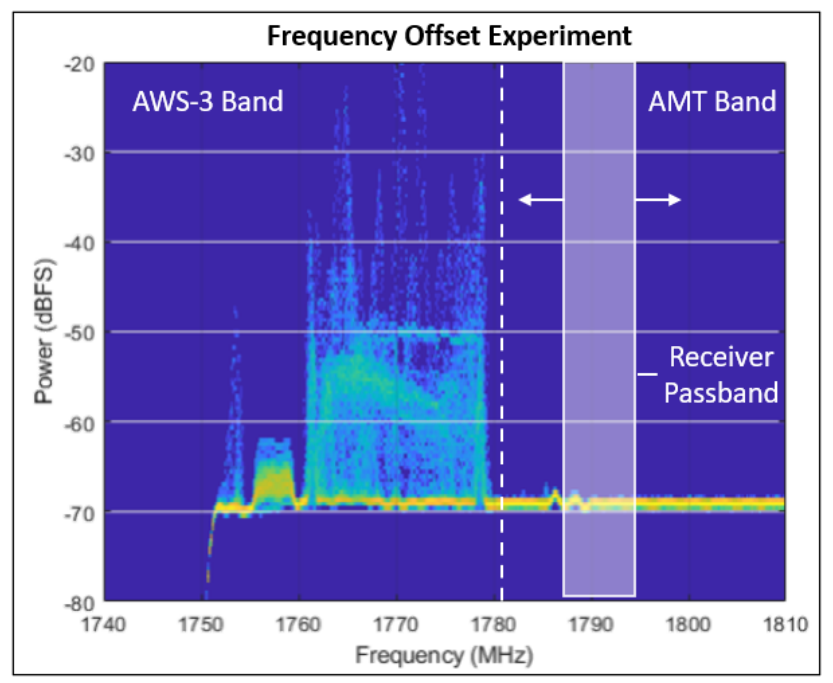

Figure 3.10: AMT and ABE frequency separation for frequency offset experiment with extended noise ABE waveform.

complex lowpass filters are applied with cutoff frequencies of $10 \mathrm{MHz}, 9 \mathrm{MHz}$ and $8.25 \mathrm{MHz}$ to create the $20 \mathrm{MHz}$, $18 \mathrm{MHz}$ and $16.5 \mathrm{MHz}$ variants of the shaped Gaussian noise waveform, respectively. During testing, the $20 \mathrm{MHz}$ and $18 \mathrm{MHz}$ waveforms are generated with a center frequency of $1770 \mathrm{MHz}$, and the $16.5 \mathrm{MHz}$ waveform is generated with a center frequency of $1769.25 \mathrm{MHz}$. Figure 3.11 shows the resulting spectrum analyzer traces for these waveforms.

\subsubsection{Summary of ABE waveforms}

The selected ABE waveforms, their classification, and origin are tabulated in Table 3.5. Note that, as discussed in previous sections, there are various degrees of filtering applied to the waveforms, altering the spectral content of the LTE out of band (OOB) portion, but preserving the in-band content. This distinction owes to the type of measurement the $\mathrm{ABE}$ waveforms are being used for. 


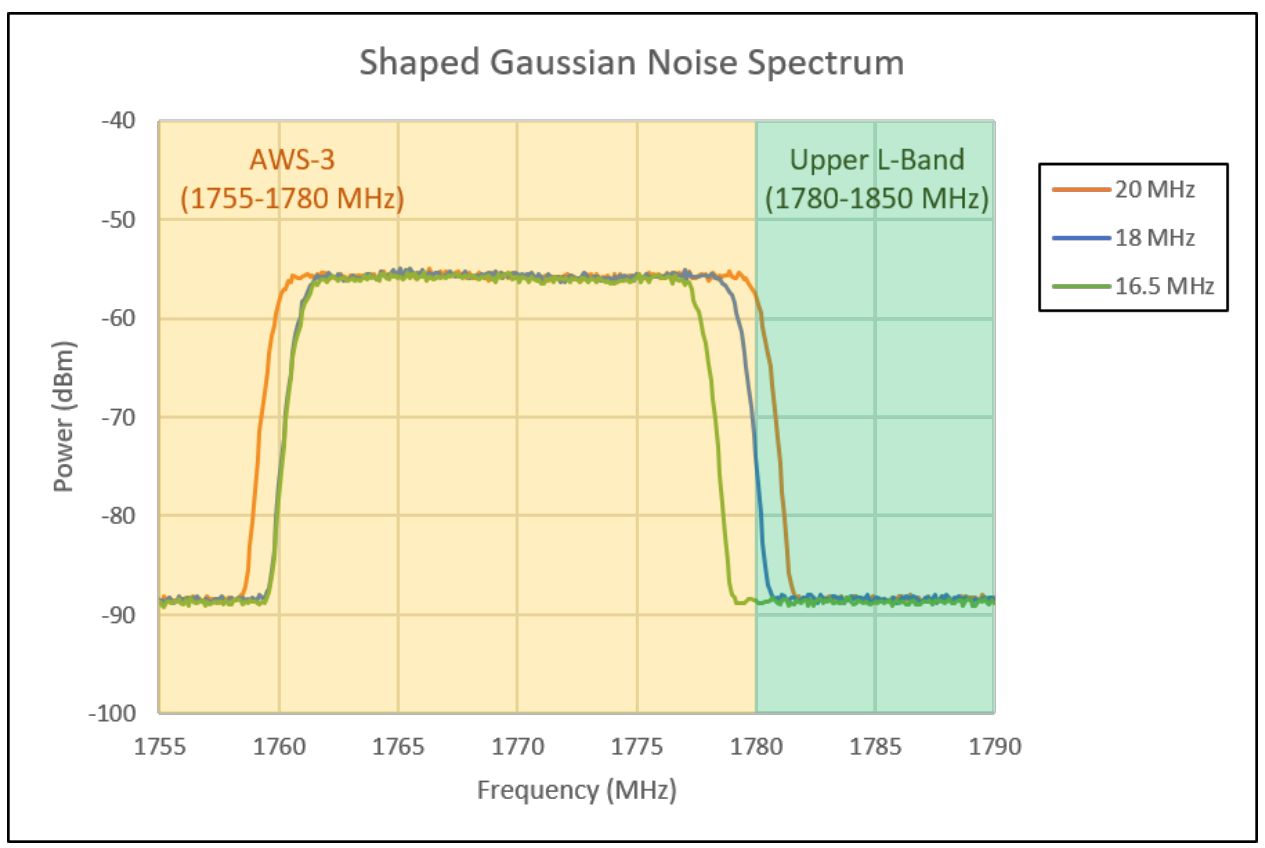

Figure 3.11: Comparison of shaped Gaussian noise spectrum. 
Table 3.1: Test equipment used in the testbed at Bedford, MA and Boulder, CO.

\begin{tabular}{|c|c|c|c|}
\hline Testbed & Item & Purpose & Instrument $^{\dagger}$ \\
\hline Boulder & $\begin{array}{l}\text { AMT TX } \\
\text { AMT RX } \\
\text { VSG } \\
\text { VSA } \\
\text { power meter } \\
\text { programmable } \\
\text { variable attenuator }\end{array}$ & $\begin{array}{l}\text { ABE waveform } \\
\text { diagnostic } \\
\text { diagnostic } \\
\text { signal level setting }\end{array}$ & $\begin{array}{l}\text { Semco/Emhiser Research } \\
\text { ESTI Series } \\
\text { Serial\# 001015D2D502-01057000 } \\
\text { Version: 03.89 } \\
\text { Quasonix } \\
\text { 3rd Gen RDMS 1U } \\
\text { Model: QSX-RDMS-3R1D-E1-1111-00-EQ-14-CS } \\
\text { Serial Number: 3636 } \\
\text { Firmware: } 16.3 \\
\text { Channel: } 2 \\
\text { Rhode \& Schwarz } \\
\text { Model: SMW200A } \\
\text { Serial Number 1412.0000K02/105322 } \\
\text { Rhode \& Schwarz } \\
\text { Model: FSW-26 } \\
\text { Serial Number: 1312.8000K26/104639 } \\
\text { Keysight Technologies } \\
\text { Model: U2044XA } \\
\text { Serial Number: SG56400003 } \\
\text { Minicircuits } \\
\text { Model: RUDAT 6000-110 }\end{array}$ \\
\hline Bedford & $\begin{array}{l}\text { AMT TX } \\
\text { AMT RX } \\
\text { VSG } \\
\text { VSA } \\
\text { power meter } \\
\text { programmable } \\
\text { variable attenuator }\end{array}$ & $\begin{array}{l}\text { ABE waveform } \\
\text { diagnostic } \\
\text { diagnostic }\end{array}$ & $\begin{array}{l}\text { Quasonix receiver analyzer } \\
\text { QSX-RXAN-281D-A1-1111 } \\
\text { Serial Number: H3590031 } \\
\text { HW Rev: B } \\
\text { Firmware Rev: RA V1.205 } \\
\text { Quasonix receiver } \\
\text { 3rd Gen RDMS 1U } \\
\text { Model: QSX-RDMS-3R1D-E1-1111-00-EQ-14-CS } \\
\text { Serial Number: 3764 } \\
\text { Firmware: 16.3 } \\
\text { Channel: 1 } \\
\text { National Instruments } \\
\text { Test Set: PXIe-5673E } \\
\text { Serial Number: F644A2 } \\
\text { Agilent Technologies } \\
\text { Model: N9020A } \\
\text { Serial Number: SG49100043 } \\
\text { ANRITSU } \\
\text { Model: ML2496A } \\
\text { Serial Number: 6K00004154 } \\
\text { Minicircuits } \\
\text { Model: RUDAT 6000-110 }\end{array}$ \\
\hline
\end{tabular}

$\dagger$ Certain commercial equipment, instruments, or materials are identified in this report in order to specify the experimental procedures adequately. Such identification is not intended to imply recommendation or endorsement by National Institute of Standards and Technology (NIST)

nor is it intended to imply that the materials or equipment identified are necessarily the best available for the purpose. 
Table 3.2: Receiver IF filter selection for various modulations and bit rates.

\begin{tabular}{|l|l|l|}
\hline Modulation & bit rate & Nominal IF Filter Bandwidth \\
\hline PCM/FM & $1 \mathrm{Mbps}$ & $2 \mathrm{MHz}$ \\
PCM/FM & $5 \mathrm{Mbps}$ & $10 \mathrm{MHz}$ \\
PCM/FM & $10 \mathrm{Mbps}$ & $20 \mathrm{MHz}$ \\
PCM/FM & $20 \mathrm{Mbps}$ & $40 \mathrm{MHz}$ \\
\hline ARTM CPM & $1 \mathrm{Mbps}$ & $1 \mathrm{MHz}$ \\
ARTM CPM & $5 \mathrm{Mbps}$ & $6 \mathrm{MHz}$ \\
ARTM CPM & $10 \mathrm{Mbps}$ & $10 \mathrm{MHz}$ \\
ARTM CPM & $20 \mathrm{Mbps}$ & $20 \mathrm{MHz}$ \\
\hline SOQPSK & $1 \mathrm{Mbps}$ & $1.4 \mathrm{MHz}$ \\
SOQPSK & $5 \mathrm{Mbps}$ & $6 \mathrm{MHz}$ \\
SOQPSK & $10 \mathrm{Mbps}$ & $14 \mathrm{MHz}$ \\
SOQPSK & $20 \mathrm{Mbps}$ & $28 \mathrm{MHz}$ \\
\hline SOQPSK LDPC $(\mathrm{K}=4096 \mathrm{R}=2 / 3)$ & $1 \mathrm{Mbps}$ & $2 \mathrm{MHz}$ \\
SOQPSK LDPC $(\mathrm{K}=4096 \mathrm{R}=2 / 3)$ & $5 \mathrm{Mbps}$ & $10 \mathrm{MHz}$ \\
SOQPSK LDPC $(\mathrm{K}=4096 \mathrm{R}=2 / 3)$ & $10 \mathrm{Mbps}$ & $20 \mathrm{MHz}$ \\
SOQPSK LDPC $(\mathrm{K}=4096 \mathrm{R}=2 / 3)$ & $20 \mathrm{Mbps}$ & $40 \mathrm{MHz}$ \\
\hline STC & $1 \mathrm{Mbps}$ & $1.4 \mathrm{MHz}$ \\
STC & $5 \mathrm{Mbps}$ & $6 \mathrm{MHz}$ \\
STC & $10 \mathrm{Mbps}$ & $14 \mathrm{MHz}$ \\
STC & $20 \mathrm{Mbps}$ & $28 \mathrm{MHz}$ \\
\hline STC LDPC $(\mathrm{K}=4096 \mathrm{R}=2 / 3)$ & $1 \mathrm{Mbps}$ & $2 \mathrm{MHz}$ \\
STC LDPC $(\mathrm{K}=4096 \mathrm{R}=2 / 3)$ & $5 \mathrm{Mbps}$ & $10 \mathrm{MHz}$ \\
STC LDPC $(\mathrm{K}=4096 \mathrm{R}=2 / 3)$ & $10 \mathrm{Mbps}$ & $20 \mathrm{MHz}$ \\
\hline
\end{tabular}


Table 3.3: $99 \%$ occupied bandwidth and corresponding minimum frequency for various AMT modulations and bit rates.

\begin{tabular}{|l|l|l|l|}
\hline Modulation & $\begin{array}{l}\text { bit rate } \\
\text { (Mbps) }\end{array}$ & $\begin{array}{l}\mathbf{9 9 \%} \text { Occupied } \\
\text { Bandwidth } \\
\text { (MHz) }\end{array}$ & $\begin{array}{l}\mathbf{F}_{\text {AMT } \mid \text { MIN }} \\
\text { (MHz) }\end{array}$ \\
\hline PCM/FM & 1 & 1.11 & 1780.56 \\
PCM/FM & 5 & 5.54 & 1782.77 \\
PCM/FM & 10 & 11.10 & 1785.55 \\
PCM/FM & 20 & 22.20 & 1791.10 \\
\hline ARTM CPM & 1 & 0.56 & 1780.28 \\
ARTM CPM & 5 & 2.83 & 1781.41 \\
ARTM CPM & 10 & 5.65 & 1782.83 \\
ARTM CPM & 20 & 11.29 & 1785.65 \\
\hline SOQPSK & 1 & 0.79 & 1780.39 \\
SOQPSK & 5 & 3.94 & 1781.97 \\
SOQPSK & 10 & 7.89 & 1783.94 \\
SOQPSK & 20 & 15.77 & 1787.88 \\
\hline SOQPSK LDPC (K=4096 R=2/3) & 1 & 1.23 & 1780.61 \\
SOQPSK LDPC (K=4096 R=2/3) & 5 & 6.14 & 1783.07 \\
SOQPSK LDPC (K=4096 R=2/3) & 10 & 12.28 & 1786.14 \\
SOQPSK LDPC (K=4096 R=2/3) & 20 & 24.64 & 1792.32 \\
\hline STC & 1 & 0.82 & 1780.41 \\
STC & 5 & 4.09 & 1782.04 \\
STC & 10 & 8.19 & 1784.10 \\
STC & 20 & 16.39 & 1788.19 \\
\hline STC LDPC (K=4096 R=2/3) & 1 & 1.28 & 1780.64 \\
STC LDPC (K=4096 R=2/3) & 5 & 6.37 & 1783.18 \\
STC LDPC (K=4096 R=2/3) & 10 & 12.76 & 1786.38 \\
\hline
\end{tabular}


Table 3.4: AMT and ABE test frequencies used in Bedford and Boulder testbeds.

\begin{tabular}{|c|c|c|c|c|c|}
\hline \multirow[b]{2}{*}{ Modulation } & \multirow[b]{2}{*}{$\begin{array}{l}\text { bit rate } \\
\text { (Mbps) }\end{array}$} & \multicolumn{2}{|c|}{ Bedford } & \multicolumn{2}{|c|}{ Boulder } \\
\hline & & $\begin{array}{l}\text { F }_{\text {AMT }} \\
\text { (MHz) }\end{array}$ & $\begin{array}{l}F_{\text {ABE }} \\
(\mathrm{MHz})\end{array}$ & $\begin{array}{l}\text { F }_{\text {AMT }} \\
(\mathrm{MHz})\end{array}$ & $\begin{array}{l}F_{\text {ABE }} \\
(\mathrm{MHz})\end{array}$ \\
\hline PCM/FM & 1 & 1780.56 & 1770 & 1780.5 & 1769.94 \\
\hline $\mathrm{PCM} / \mathrm{FM}$ & 5 & 1782.77 & 1770 & 1782.75 & 1769.98 \\
\hline $\mathrm{PCM} / \mathrm{FM}$ & 10 & 1785.55 & 1770 & 1785.5 & 1769.95 \\
\hline $\mathrm{PCM} / \mathrm{FM}$ & 20 & 1791.10 & 1770 & 1791 & 1769.9 \\
\hline ARTM CPM & 1 & 1780.28 & 1770 & 1780.25 & 1769.97 \\
\hline ARTM CPM & 5 & 1781.41 & 1770 & 1781.5 & 1770.09 \\
\hline ARTM CPM & 10 & 1782.83 & 1770 & 1782.75 & 1769.92 \\
\hline ARTM CPM & 20 & 1785.65 & 1770 & 1785.75 & 1770.1 \\
\hline SOQPSK & 1 & 1780.39 & 1770 & 1780.5 & 1770.11 \\
\hline SOQPSK & 5 & 1781.97 & 1770 & 1782 & 1770.03 \\
\hline SOQPSK & 10 & 1783.94 & 1770 & 1784 & 1770.06 \\
\hline SOQPSK & 20 & 1787.88 & 1770 & 1788 & 1770.12 \\
\hline SOQPSK LDPC $(\mathrm{K}=4096 \mathrm{R}=2 / 3)$ & 1 & 1780.61 & 1770 & N/A & N/A \\
\hline SOQPSK LDPC $(\mathrm{K}=4096 \mathrm{R}=2 / 3)$ & 5 & 1783.07 & 1770 & N/A & N/A \\
\hline SOQPSK LDPC $(K=4096 \mathrm{R}=2 / 3)$ & 10 & 1786.14 & 1770 & N/A & N/A \\
\hline SOQPSK LDPC $(K=4096 \mathrm{R}=2 / 3)$ & 20 & 1792.32 & 1770 & N/A & N/A \\
\hline STC & 1 & 1780.41 & 1770 & N/A & N/A \\
\hline STC & 5 & 1782.04 & 1770 & N/A & N/A \\
\hline STC & 10 & 1784.10 & 1770 & N/A & N/A \\
\hline STC & 20 & 1788.19 & 1770 & N/A & N/A \\
\hline STC LDPC $(\mathrm{K}=4096 \mathrm{R}=2 / 3)$ & 1 & 1780.64 & 1770 & N/A & N/A \\
\hline STC LDPC $(K=4096 \mathrm{R}=2 / 3)$ & 5 & 1783.18 & 1770 & N/A & N/A \\
\hline STC LDPC $(K=4096 \mathrm{R}=2 / 3)$ & 10 & 1786.38 & 1770 & N/A & N/A \\
\hline
\end{tabular}


Table 3.5: ABE waveforms names, properties, and experiments

\begin{tabular}{|c|c|c|c|c|}
\hline Name & Type & Source & Experiments & Notes \\
\hline AWGN $20 \mathrm{MHz}$ & $20 \mathrm{MHz}$ wide AWGN & DSP generated & $\begin{array}{l}\text { Main, Frequency Offset, } \\
\text { Side A, \& Side B }\end{array}$ & matches $20 \mathrm{MHz}$ LTE allocation \\
\hline AWGN $18 \mathrm{MHz}$ & $18 \mathrm{MHz}$ wide AWGN & DSP generated & Main, Side A, \& Side B & matches bandwidth of $100 \mathrm{RBs}$ \\
\hline AWGN $16.5 \mathrm{MHz}$ & 16.5 MHz wide AWGN & DSP generated & Main, Side A, \& Side B & approx. bandwidth of lower $92 \mathrm{RBs}$ \\
\hline $\begin{array}{l}\text { Single-UE Full } 100 \mathrm{RB} \\
\text { (EAFB, } 20 \mathrm{MHz} \text { Filter) }\end{array}$ & $\begin{array}{l}20 \mathrm{MHz} \text { LTE allocation, full } \\
\text { RB usage }\end{array}$ & $\begin{array}{l}\text { IQ capture at EAFB; filtered for } \\
\text { LTE in-band portion }\end{array}$ & Main, Side A, \& Side B & $\begin{array}{l}34 \text { TTI waveform snippet of UE } \\
\text { streaming at } 10 \mathrm{Mbps}\end{array}$ \\
\hline $\begin{array}{l}\text { Single-UE Lower } 92 \mathrm{RB} \\
\text { (EAFB, } 20 \mathrm{MHz} \text { Filter) }\end{array}$ & $\begin{array}{l}20 \mathrm{MHz} \text { LTE allocation, ac- } \\
\text { tivity in lower } 92 \mathrm{RB}\end{array}$ & $\begin{array}{l}\text { IQ capture at EAFB; filtered for } \\
\text { LTE in-band portion }\end{array}$ & Main, Side A, \& Side B & $\begin{array}{l}30 \mathrm{TTI} \text { waveform of UE streaming } \\
\text { at } 10 \mathrm{Mbps}\end{array}$ \\
\hline $\begin{array}{l}\text { Single-UE Full } 100 \mathrm{RB} \\
\text { (EAFB, } 20 \mathrm{MHz} \text { Filter) }\end{array}$ & $\begin{array}{l}20 \mathrm{MHz} \text { LTE allocation, full } \\
\text { RB usage }\end{array}$ & $\begin{array}{l}\text { IQ capture at EAFB; LTE in- } \\
\text { band \& ext. noise floor }\end{array}$ & Frequency Offset & $\begin{array}{l}\text { Main experiment version with ex- } \\
\text { tended noise floor }\end{array}$ \\
\hline $\begin{array}{r}\text { Single-UE Lower } 92 \mathrm{RB} \\
\text { (EAFB, } 20 \mathrm{MHz} \text { Filter) }\end{array}$ & $\begin{array}{l}20 \mathrm{MHz} \text { LTE allocation, ac- } \\
\text { tivity in lower } 92 \mathrm{RB}\end{array}$ & $\begin{array}{l}\text { IQ capture at EAFB; LTE in- } \\
\text { band \& ext. noise floor }\end{array}$ & Frequency Offset & $\begin{array}{l}\text { Main experiment version with ex- } \\
\text { tended noise floor }\end{array}$ \\
\hline $\begin{array}{l}\text { Single-UE Full } 100 \text { RB } \\
\text { (EAFB, } 37 \text { MHz Filter) }\end{array}$ & $\begin{array}{l}20 \mathrm{MHz} \text { LTE allocation, full } \\
\text { RB usage }\end{array}$ & $\begin{array}{l}\text { IQ capture at EAFB; LTE in- } \\
\text { band, OOBE \& ext. noise floor }\end{array}$ & Frequency Offset & \\
\hline $\begin{array}{r}\text { Single-UE Lower } 92 \mathrm{RB} \\
\text { (EAFB, } 37 \mathrm{MHz} \text { Filter) }\end{array}$ & $\begin{array}{l}20 \mathrm{MHz} \text { LTE allocation, ac- } \\
\text { tivity in lower } 92 \mathrm{RB}\end{array}$ & $\begin{array}{l}\text { IQ capture at EAFB; LTE in- } \\
\text { band, OOBE \& ext. noise floor }\end{array}$ & Frequency Offset & \\
\hline $\begin{array}{r}\text { Multi-UE Az-76 } 100 \mathrm{RB} \\
(\mathrm{EAFB}, 20 \mathrm{MHz} \text { Filter })\end{array}$ & $\begin{array}{l}20 \mathrm{MHz} \text { LTE allocation, full } \\
\text { RB usage }\end{array}$ & $\begin{array}{l}\text { IQ capture at EAFB; filtered for } \\
\text { LTE in-band portion }\end{array}$ & Main, Side A, \& Side B & $\begin{array}{l}\text { AMT asset antenna trained at } \\
76^{\circ} \text { azimuth, } 0^{\circ} \text { horizon }\end{array}$ \\
\hline $\begin{array}{r}\text { Multi-UE Az-198 } 100 \mathrm{RB} \\
(\mathrm{EAFB}, 20 \mathrm{MHz} \text { Filter })\end{array}$ & $\begin{array}{l}20 \mathrm{MHz} \text { LTE allocation, full } \\
\text { RB usage }\end{array}$ & $\begin{array}{l}\text { IQ capture at EAFB; filtered for } \\
\text { LTE in-band portion }\end{array}$ & Main, Side A, \& Side B & $\begin{array}{l}\text { AMT asset antenna trained at } \\
198^{\circ} \text { azimuth, } 0^{\circ} \text { horizon }\end{array}$ \\
\hline $\begin{array}{r}\text { Multi-UE Az-140 } 100 \mathrm{RB} \\
(\text { LARC, } 20 \mathrm{MHz} \text { Filter })\end{array}$ & $\begin{array}{l}20 \mathrm{MHz} \text { LTE allocation, full } \\
\text { RB usage }\end{array}$ & $\begin{array}{l}\text { IQ capture at LARC; filtered for } \\
\text { LTE in-band portion }\end{array}$ & Main, Side A, \& Side B & $\begin{array}{l}\text { AMT asset antenna trained at } \\
140^{\circ} \text { azimuth, } 0^{\circ} \text { horizon }\end{array}$ \\
\hline $\begin{array}{r}\text { Multi-UE Az- } 165^{\circ} 100 \mathrm{RB} \\
\text { (LARC, } 20 \mathrm{MHz} \text { Filter) }\end{array}$ & $\begin{array}{l}20 \mathrm{MHz} \text { LTE allocation, full } \\
\text { RB usage }\end{array}$ & $\begin{array}{l}\text { IQ capture at LARC; filtered for } \\
\text { LTE in-band portion }\end{array}$ & Main, Side A, \& Side B & $\begin{array}{l}\text { AMT asset antenna trained at } \\
165^{\circ} \text { azimuth, } 0^{\circ} \text { horizon }\end{array}$ \\
\hline $\begin{array}{r}\text { Multi-UE Az-76 } 100 \mathrm{RB} \\
(\mathrm{EAFB}, 37 \mathrm{MHz} \text { Filter })\end{array}$ & $\begin{array}{l}20 \mathrm{MHz} \text { LTE allocation, full } \\
\text { RB usage }\end{array}$ & $\begin{array}{l}\text { IQ capture at EAFB; LTE in- } \\
\text { band, OOBE \& ext. noise floor }\end{array}$ & Frequency Offset & \\
\hline $\begin{array}{r}\text { Multi-UE Az-198 } 100 \mathrm{RB} \\
(\mathrm{EAFB}, 20 \mathrm{MHz} \text { Filter })\end{array}$ & $\begin{array}{l}20 \mathrm{MHz} \text { LTE allocation, full } \\
\text { RB usage }\end{array}$ & $\begin{array}{l}\text { IQ capture at EAFB; LTE in- } \\
\text { band, OOBE \& ext. noise floor }\end{array}$ & Frequency Offset & \\
\hline $\begin{array}{r}\text { Single-UE Full 100 RB } \\
\text { (Lab, } 49 \mathrm{MHz} \text { Filter) }\end{array}$ & $\begin{array}{l}20 \mathrm{MHz} \text { LTE allocation, full } \\
\text { RB usage }\end{array}$ & $\begin{array}{l}\text { IQ capture at NBIT; LTE in- } \\
\text { band, OOBE \& ext. noise floor }\end{array}$ & Frequency Offset & UE streaming at $10 \mathrm{Mbps}$ \\
\hline $\begin{array}{r}\text { Single-UE Lower } 90 \mathrm{RB} \\
\text { (Lab, } 49 \mathrm{MHz} \text { Filter) }\end{array}$ & $\begin{array}{l}20 \mathrm{MHz} \text { LTE allocation, } \\
\text { lower } 90 \mathrm{RB} \text { activity }\end{array}$ & $\begin{array}{l}\text { IQ capture at NBIT; LTE in- } \\
\text { band, OOBE \& ext. noise floor }\end{array}$ & Frequency Offset & $\begin{array}{l}\text { RB blanking eNB function; UE } \\
\text { streaming at } 10 \mathrm{Mbps}\end{array}$ \\
\hline $\begin{array}{r}\text { Single-UE Full } 25 \mathrm{RB} \text { (Lab, } \\
49 \mathrm{MHz} \text { Filter) }\end{array}$ & $\begin{array}{l}5 \mathrm{MHz} \text { LTE allocation, full } \\
\text { RB usage }\end{array}$ & $\begin{array}{l}\text { IQ capture at NBIT; LTE in- } \\
\text { band, OOBE \& ext. noise floor }\end{array}$ & Frequency Offset & UE streaming at $1 \mathrm{Mbps}$ \\
\hline
\end{tabular}




\subsection{Test Automation}

The data collected for this study is encompasses 10,222 individual measurements made over a six month period at two locations. The measurements require the coordination of a number of pieces of test equipment with many critical parameters to set. To ensure consistency of the measurement process, minimize opportunities for human error and optimize the use of the available test time, the test process is fully automated. This section describes the testbed software architecture and test process.

\subsubsection{Software Architecture}

The test automation software is built using a MITRE-developed test automation framework (TAF) used for several previous measurement campaigns. The framework provides a standard process flow and extensible instrumentation interface that can be applied to a wide variety of automated measurement applications.

Figure 3.12 shows the TAF system architecture and top level process flow for a test run. Test runs are configured by two input files: the Test Configuration File (TCF) and the Test Parameter File (TPF). The TCF defines the test instruments to be used in the run along with their initial conditions. It also specifies general settings for the test run, such as the location of the output data and the option to enable (or disable) the randomization of the test execution order. During the initialization process the system reads this file and initializes itself accordingly. This includes establishing communication with all instruments and configuring them to the specified initial conditions.

The TPF defines the unique parameters for each individual test. For each iteration of the test execution process, the framework reads one line from this file and configures the instruments as specified. The framework then executes the test and saves the output data to a dedicated subfolder in the output folder structure. When execution of all tests in the TPF are complete, the test run ends.

Interfaces between the framework and test instruments, known as components, enable the framework to configure the instruments. The components also enable the instruments to optionally provide output data to the framework, as well as status information. TAF components are developed for all instruments listed in Table 3.1. In cases where different instruments are used in the Boulder and Bedford testbeds, separate versions of the components are developed. The following is an overview of the components:

\section{- AMT Receiver}

The AMT receiver serves as the reference receiver in the testbed. The TAF AMT receiver component interfaces with the receiver to configure parameters of the AMT signal such as frequency, modulation and bit rate, as well as receiver settings such as AGC time constant and equalizer state. It also configures parameters for the receiver's built-in BER measurement capability, which include the pseudorandom data pattern and measurement length. The component outputs a configurable list of receiver system status parameters (also referred to as KPIs). These parameters are reported as time series with a $200 \mathrm{~ms}$ period. A complete list of KPIs is provided in Table G.1.

\section{- AMT Transmitter}

The AMT transmitter generates the reference AMT signal in the testbed. The TAF AMT transmitter component interfaces with the transmitter to configure parameters of the AMT waveform such as frequency, modulation, bit rate, pseudorandom data pattern and output power level. There are two versions of the component to support the different instruments used in the Bedford and Boulder testbeds, both with a common set of configuration parameters.

\section{- Vector Signal Generator}

The VSG is used in the testbed to generate various ABE waveforms. Inputs to the component include the waveform filename, I/Q sample rate, output frequency and output power level. There are two versions of the TAF VSG component due to the different instruments used in the Bedford and Boulder testbeds. 


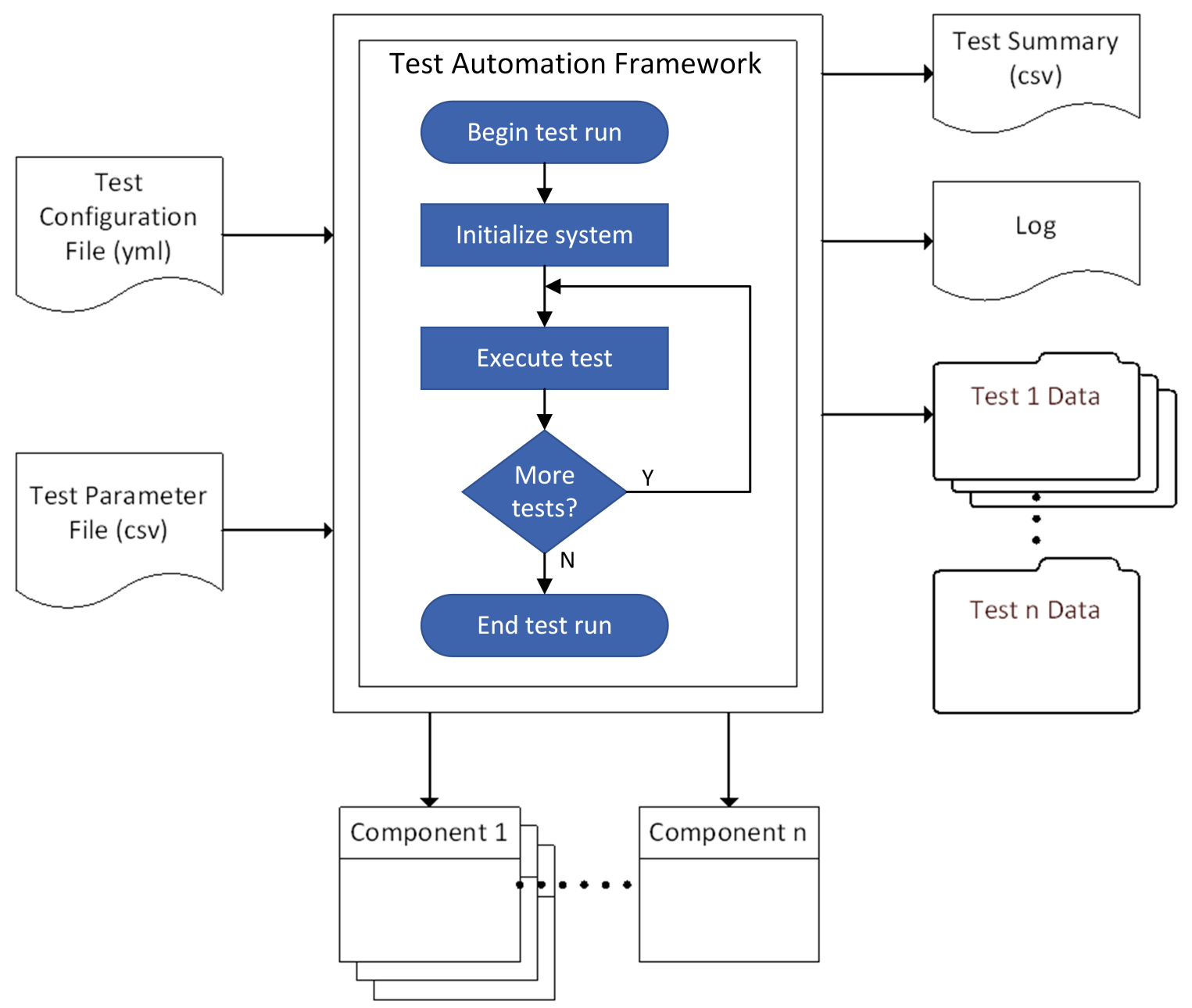

Figure 3.12: Test automation framework architecture and top-level process flow. 


\section{- Vector Signal Analyzer}

The VSA is used to capture the power spectra of the AMT and ABE signals at certain stages of the test process. This data is used to monitor health of the testbed and to help diagnose issues. The component configures the instrument's center frequency, span, resolution bandwidth and reference level, and returns the power spectrum. The power spectrum is provided by the component as both trace data and an instrument screen capture. There are two versions of the TAF VSA component due to the difference in VSAs used in the Bedford and Boulder testbeds.

\section{- Power Meter}

The power meter is used to measure the average power of the AMT and ABE reference signals at certain stages of the test process. These measurements are combined with known losses in the signal paths to determine signal power levels at the plane of the receiver. The components configure the measurement settings such as measurement units, calibration factor frequency and averaging settings, and return the measured average power. There are two versions of the TAF power meter components due to the different instruments used in the Bedford and Boulder testbeds.

\section{- Programmable Attenuator}

Two programmable attenuators are used in the testbed to set the power levels of the AMT and ABE signals at the plane of the receiver. The TAF attenuator component configures the attenuators to the nearest actual values to those requested by the system, using calibration data specific to each device. The component returns the actual attenuation values per the calibration data.

\subsection{Test Process}

The overall test process can be broken down into two levels: a test and a run. A test is a single measurement with a fixed set of test conditions. A run is a series of tests, optionally executed in random order, with unique test conditions for each iteration. The framework provides complete flexibility to vary any number of test parameters on a per-test basis and execute any number of tests in a run. The following sections describe the process for an individual test execution, and how tests are organized into runs.

\subsubsection{Run Organization}

The framework provides complete flexibility to vary any number of test parameters on a per-test basis. However, the time required to configure the various instruments must be considered to optimize overall test time. For this experiment, configuration changes to the receiver and VSG are known to be costly in terms of test execution time, whereas changes to the attenuators are known to be inexpensive. Therefore it is advantageous to minimize configuration changes to the receiver and VSG during the run. The tests are organized into runs separately for the main experiment and frequency offset experiment with this in mind.

\subsubsection{Main Experiment}

Main experiment test runs are organized such that the time-intensive configuration operations, namely the receiver and VSG configuration, are required just once at the beginning of the run. During the iterative test executions only the programmable attenuators require reconfiguration on a per-test basis.

At the start of each run, prior to test execution, the AMT receiver, AMT transmitter and VSG are configured according to the test configuration file. These settings remain fixed for the duration of the test run. The unique parameters for each test execution and the order of test execution are defined in the test parameter file. For each test iteration in the run, the framework reads a single line from the test parameter file and configures the system accordingly. Table 3.6 is an example of test parameters for a main experiment test run.

The tests begin with reference power measurements of the AMT and ABE signals (test_num 1 and 2). For these measurements the attenuator for the signal being measured is set to $0 \mathrm{~dB}$ to maximize the power level and ensure that 
Table 3.6: Example main experiment parameter file.

\begin{tabular}{|l|l|l|l|l|}
\hline test_num & attn_amt & attn_abe & pow_met.disabled & randomize \\
\hline 1 & 0 & 110 & FALSE & FALSE \\
\hline 2 & 110 & 0 & FALSE & FALSE \\
\hline 3 & $\begin{array}{l}\text { Baseline } 1 \\
\text { (no ABE) }\end{array}$ & 110 & TRUE & FALSE \\
\hline 4 & $\begin{array}{l}\text { Baseline } 2 \\
\text { (no ABE) }\end{array}$ & 110 & TRUE & FALSE \\
\hline 5 & AMT Level 1 & Fixed ABE Level & TRUE & TRUE \\
\hline 6 & AMT Level 2 & Fixed ABE Level & TRUE & TRUE \\
\hline$\vdots$ & $\vdots$ & $\vdots$ & $\vdots$ & $\vdots$ \\
\hline $\mathrm{X}$ & AMT Level N & Fixed ABE Level & TRUE & TRUE \\
\hline $\mathrm{Y}$ & 0 & 110 & FALSE & FALSE \\
\hline $\mathrm{Z}$ & 110 & 0 & FALSE & FALSE \\
\hline
\end{tabular}

the signal power within the measurement range of the power meter. The attenuator for the other signal is set to the maximum value of $110 \mathrm{~dB}$ so that it does not impact the measurement. The setting of pow_met.disabled $=$ FALSE in the test parameter file causes the testbed to execute a power measurement. The setting of randomize $=$ FALSE forces the testbed to execute these tests in the order specified in the file. Following the reference power measurements, two baseline measurements are performed with no ABE signal present (test_num 3 and 4). The baseline measurements collect receiver KPIs with a known AMT signal level and no ABE signal, and are used for diagnostic purposes. As with the reference power measurements, these tests execute in a fixed order also. After the four verification measurements the randomized test run begins (test_num 5 through $\mathrm{X}$ ). $N$ tests are executed in random order, with the AMT signal at various power levels and the ABE signal at a fixed level. Finally an additional set of reference power measurements are made at the end of the run (test_num $\mathrm{Y}$ and $\mathrm{Z}$ ) to monitor for any drift in signal power levels.

Prior to running the main experiment, data is manually captured to characterize the onset of a measurable BER, e.g., a BER on the order of $10^{-6}$, as a function of the AMT signal attenuation for the full set of permutations of modulation, bit rate, and $\mathrm{ABE}$ waveform. The attenuation levels where these transition points occur, along with their respective whole plot variables, are then fed to an automatic configuration file generator. The configuration file generator created unique TPFs for each whole plot configuration, with the measurement points expanded around the transition point.

\subsubsection{Frequency Offset Experiment}

The frequency offset experiment differs from the main experiment in that the AMT signal level remains constant while the AMT frequency offset from the band-edge is varied on a per-test basis. At the start of each run, prior to test execution, the AMT receiver, AMT transmitter and VSG are still configured according the test configuration file. However, since the AMT receiver and AMT transmitter frequencies change for each test iteration, the TPF includes fields that specify the unique frequency values per test. Aside from that, the test run organization for the frequency offset experiment is largely the same as for the main experiment. Table 3.7 is an example of test parameters for a frequency experiment test run.

As in the main experiment, a frequency offset experiment test run begins with two power measurement tests (test_num 1 and 2) and two baseline measurements (test_num 3 and 4). For these tests the AMT frequency is set to the minimum value per Table 3.4, while the AMT and ABE attenuators are set to the specified values for each measurement. After the four verification measurements the randomized test run begins (test_num 5 through $\mathrm{X}$ ). $N$ tests are executed in random order, with the AMT and ABE signals at fixed power levels, and the AMT frequency is varied. An additional set of reference power measurements are made at the end of the run (test_num $\mathrm{Y}$ and $\mathrm{Z}$ ). 
Table 3.7: Example frequency offset experiment parameter file.

\begin{tabular}{|l|l|l|l|l|l|l|}
\hline test_num & attn_amt & attn_abe & $\begin{array}{l}\text { amtrx. } \\
\text { frequency }\end{array}$ & $\begin{array}{l}\text { amttx. } \\
\text { frequency }\end{array}$ & $\begin{array}{l}\text { pow_met. } \\
\text { disabled }\end{array}$ & randomize \\
\hline 1 & 0 & 110 & $F_{A M T \mid M I N}$ & $F_{A M T \mid M I N}$ & FALSE & FALSE \\
\hline 2 & 110 & 0 & $F_{A M T \mid M I N}$ & $F_{A M T \mid M I N}$ & FALSE & FALSE \\
\hline 3 & $\begin{array}{l}\text { Baseline 1 } \\
\text { (no ABE) }\end{array}$ & 110 & $F_{A M T \mid M I N}$ & $F_{A M T \mid M I N}$ & FALSE & FALSE \\
\hline 4 & $\begin{array}{l}\text { Baseline 2 } \\
\text { (no ABE) }\end{array}$ & 110 & $F_{A M T \mid M I N}$ & $F_{A M T \mid M I N}$ & FALSE & FALSE \\
\hline 5 & $\begin{array}{l}\text { Fixed AMT } \\
\text { Level }\end{array}$ & $\begin{array}{l}\text { Fixed ABE } \\
\text { Level }\end{array}$ & AMT Freq 1 & AMT Freq 1 & TRUE & TRUE \\
\hline 6 & $\begin{array}{l}\text { Fixed AMT } \\
\text { Level }\end{array}$ & $\begin{array}{l}\text { Fixed ABE } \\
\text { Level }\end{array}$ & AMT Freq 2 & AMT Freq 2 & TRUE & TRUE \\
\hline$\vdots$ & $\vdots$ & $\vdots$ & $\vdots$ & $\vdots$ & $\vdots$ & $\vdots$ \\
\hline $\mathrm{X}$ & $\begin{array}{l}\text { Fixed AMT } \\
\text { Level }\end{array}$ & $\begin{array}{l}\text { Fixed ABE } \\
\text { Level }\end{array}$ & AMT Freq N & AMT Freq N & TRUE & TRUE \\
\hline $\mathrm{Y}$ & 0 & 110 & $F_{A M T \mid M I N}$ & $F_{A M T \mid M I N}$ & FALSE & FALSE \\
\hline $\mathrm{Z}$ & 110 & 0 & $F_{A M T \mid M I N}$ & $F_{A M T \mid M I N}$ & FALSE & FALSE \\
\hline
\end{tabular}

Prior to testing, manual measurements are performed in order to determine the minimum AMT frequency offset at which the ABE signal no longer had an impact on the AMT BER. This frequency is used as the upper frequency limit of the test run, and additional test points covering the range of frequency values are added. The configuration file generator is used to create the TCF and TPFs.

\subsubsection{Test Execution Process}

For each test iteration in a run, the same test execution process is followed. Figure 3.13 shows the process for a main experiment test. The test execution process can be described in 4 phases:

1. Initialize testbed under ideal conditions

2. Check for errors in configuration and assess data streaming capability, reboot if necessary

3. Level set AMT signal to test level and evaluate if it results in a valid test

4. Level set $\mathrm{ABE}$ to test level and record data

Initialization Each iteration of the test execution begins by setting the AMT and ABE attenuators to their test initialization values. These settings are selected such that the initialization sequence exhibits a high signal to noise ratio (SNR) and negligible $\mathrm{ABE}$ level at the plane of the receiver, this ensures that we intialize the testbed under optimal optimal receiver performance conditions. These initial values are configurable in the TCF, but for these experiments the AMT attenuator was always initialized to $30 \mathrm{~dB}$ and the $\mathrm{ABE}$ attenuator to $110 \mathrm{~dB}$.

Error check and resolution Next, on the first test of a test run the receiver is rebooted, and for subsequent tests reboot occurs as needed. This step is necessary to resolve a known issue with the receiver that results in erroneous characters being sent over the Telnet interface, which corrupts the KPI data logged by the testbed. In addition to the reboot the TAF mitigates data corruption. Examples of observed data corruption include a letter appearing where a number is expected, or an incorrect number of field separators (commas). For occasional erroneous characters the TAF inspects the incoming alphanumeric data, discards lines containing invalid characters, and logs the number of invalid lines. However, we've observed the data streaming error to worsen until the TAF obtains little or no valid data. To recover, the receiver is rebooted. As the TAF receiver component logs KPI data during each iteration of the test execution, the number of invalid lines of data is counted. When the number of invalid lines reaches a specified threshold, the receiver will be rebooted on the next iteration. 


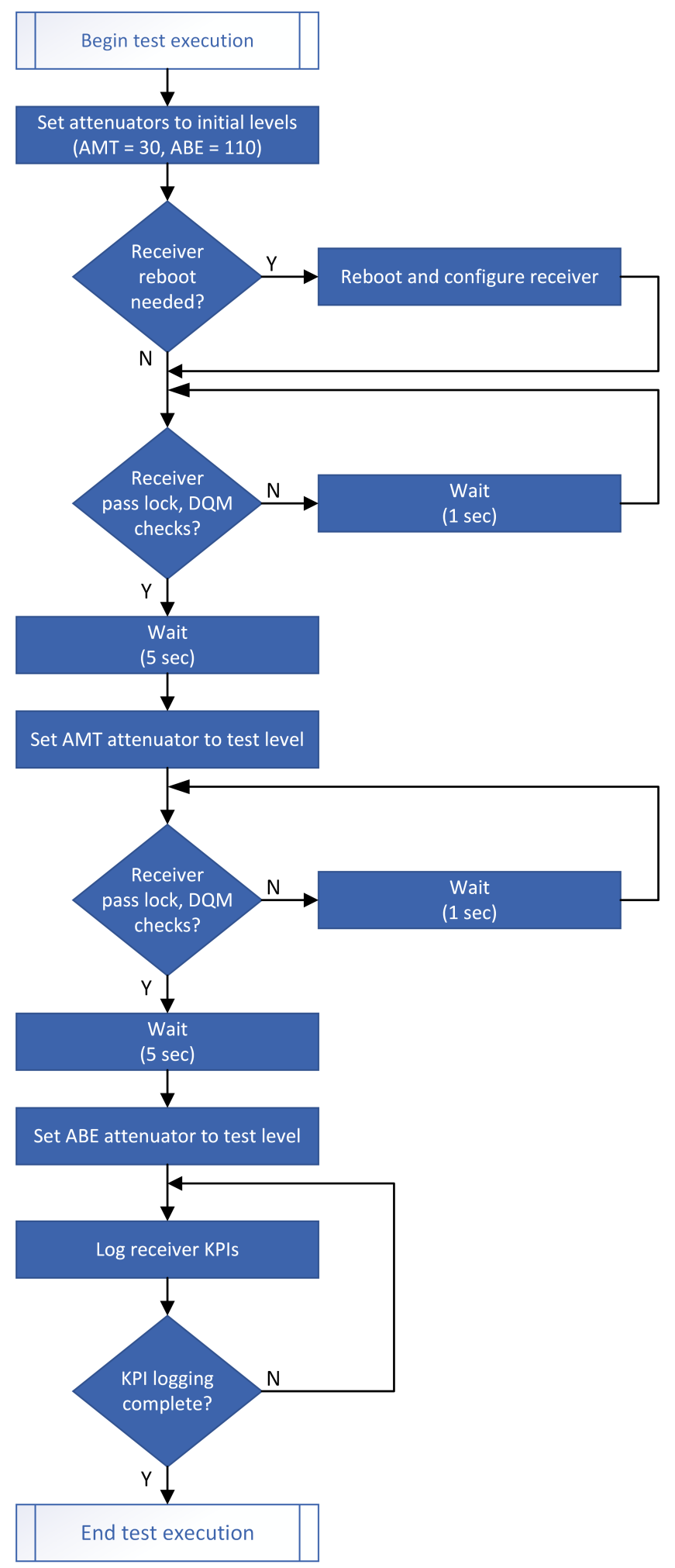

Figure 3.13: Main experiment test execution process flow. 
With the signals set to their initialization levels, the TAF receiver component next confirms that the receiver is reporting positive signal lock and that the data quality metric (DQM) exceeds a specified threshold. DQM is a proprietary method developed by Quasonix that enables the receiver to determine bit error probability (BEP) in real time. Further details can be found in the Quasonix RDMS Installation and Operation Manual [13]. During the initialization phase the receiver should always report positive signal lock and an ideal DQM of 10. While not shown in Figure 3.13, the signal lock and DQM check is set to time out if positive signal lock and DQM of 10 are not met within $5 \mathrm{~s}$. A timeout indicates an abnormal condition, and causes the entire test run to be aborted. Once conditions are met, the TAF component waits an additional $5 \mathrm{~s}$ before proceeding.

Test level evaluation The TAF receiver component then sets the AMT attenuator to the unique value specified for the test, while leaving the ABE attenuator at the initialization level. This results in a lower SNR at the plane of the receiver, but still with a negligible ABE level. The TAF component again establishes a DQM check and waits for the receiver to report positive signal lock and a DQM threshold of 2 or above. Note that measurements involving AMT modulations with FEC required for lowering the DQM to 0 in order to obtain sufficient data to establish test curves.

The TAF component will wait and check several times until these conditions are met, and then wait an additional $5 \mathrm{~s}$ before proceeding. If conditions are not met within the timeout period, the current test iteration is aborted and the framework will proceed to the next test in the run. Unlike the prior signal lock and DQM check (with the AMT signal set to its initialization level), a timeout at this point in the process does not indicate an abnormal receiver condition. It simply means that the SNR for this particular test is too low, and there is no reason to proceed because the data would have little experimental value.

Test execution Following the receiver settling period with only the AMT signal present, the ABE signal is introduced to the receiver. At this point the correct signal to adjacent band emitter power ratio (SABE) test condition exists at the plane of the receiver. The TAF component immediately starts logging KPI data from the receiver, and continues to do so until the test is complete. The overall KPI logging period is driven by the length of the BER measurement. The BER measurement continues until a specified number of bits has been transmitted. The number of bits is configurable, but for this experiment the BER measurement length is always $3 \times 10^{8}$ bits. The resulting KPI logging period ranges from $300 \mathrm{~s}$ for the lower bit rate tested (1 Mbps) to $15 \mathrm{~s}$ for the highest bit rate tested (20 Mbps). When the BER measurement is complete, a flag in the receiver KPI data changes state and the TAF component stops logging data. At this point the test execution is complete, and the framework proceeds to the next test iteration.

For BER tests, it is important to balance the individual test length (total bits) with BER measurement fidelity. The main experiment objective is to characterize conditions where BER transitions from $10^{-4}$ (a poor link) to $10^{-7}$ (a strong link), where a BER of $10^{-7}$ represents a 100 -fold decrease from the $10^{-5}$ BER threshold value. An individual test length of $3 \times 10^{8}$ bits allows for a reasonable confidence interval length in the BER range of interest. However, relative precision decreases considerably at BERs less than $10^{-7}$. A good rule of thumb is to size the test, i.e., select the total number of bits, so that the lowest BER of interest results in at least 30 bit errors on average. For example, 30 bit errors in $3 \times 10^{8}$ total bits results in a BER of $10^{-7}$.

Frequency Offset Experiment The text execution process is largely the same for the frequency offset experiment, except each test requires a unique AMT frequency. Therefore both the AMT receiver and transmitter are reconfigured to the required frequency at the start of each iteration of the test execution.

\subsubsection{Testbed data output}

The following is a summary of data products generated by the testbed. Data products can be either run-level (one data product for the entire test run) or test-level (one data product for each test iteration).

\section{- Receiver KPI Log Files (Test-Level)}

During each test iteration the receiver outputs a configurable list of KPIs. The KPIs are reported every $200 \mathrm{~ms}$ for the duration of test and saved to a plain text file. A complete list of KPIs collected in this experiment is provided in 


\section{Table G.1.}

\section{- Spectrum Analyzer Data (Test-Level)}

The spectrum analyzer in the testbed is used to monitor the signal near the input plane of the receiver. For each test iteration the spectrum analyzer data can optionally be saved. The spectrum analyzer data includes both a capture of the screen image in portable network graphics (PNG) format and the trace data in plain text format. For normal tests the spectrum analyzer measurement is disabled because the AMT and ABE signals levels at the plane of the receiver are below the noise floor of the instrument. However, test runs typically include several tests where the $\mathrm{AMT}$ or $\mathrm{ABE}$ attenuator is set to $0 \mathrm{~dB}$ to produce stronger signals (test_num $1,2, \mathrm{Y}$ and $\mathrm{Z}$ in the example test parameter files given in Tables 3.6 and 3.7). These tests allow spectrum analyzer data to be obtained to support data analysis.

\section{- Receiver Configuration Files (Test-Level)}

At the start of each test iteration the TAF receiver component queries the receiver for the states of its configurable settings. These settings are saved in a plain text file, which is primarily used for diagnostic purposes.

\section{- Test Summary File (Run-Level)}

A test summary file in plain text format is generated for the test run. Each line of the test summary file lists the inputs and outputs for each TAF component for a single test iteration. Component inputs captured in the test summary file include AMT settings such as frequency, modulation and bit rate; VSG settings such as waveform, frequency and sample rate; and settings for both attenuators. Component outputs include receiver KPI data, power meter measurements and actual attenuator values based on calibration data. Since the KPI data is provided by the receiver in the form of a time series for each test iteration, each KPI must be reduced to a single value to report in the test summary. The value reported in the test summary is either the median or final value of the time series, whichever is more relevant for each particular KPI.

The same data reported in the test summary file is also entered into a SQLite database. This provides an alternate means to aggregate data across test runs, facilitating data analysis.

\section{- TAF Test Configuration File (Run-Level)}

The test configuration file is an input to the test automation framework that defines the test instruments to be used in the run along with their initial conditions. It also specifies general settings for the test run, such as the location of the output data and the option to enable (or disable) the randomization of the test execution order. A copy of this file is saved with the test run for archival purposes.

\section{- TAF Test Parameter File (Run-Level)}

The test parameter file is an input to the test automation framework that defines the unique parameters for each individual test. For each iteration of the test execution process, the framework reads one line from this file and configures the instruments as specified. The test parameters for each individual iteration of the test are saved separately in the test-level test parameter file. A copy of this file is saved with the test run for archival purposes.

\section{- TAF Log File (Run-Level)}

Information produced by the framework during test execution which includes execution status, test equipment status, warning messages and error messages is saved to a log file. This information is intended for system diagnostic purposes. 


\section{Chapter 4}

\section{Data Analysis Methods}

\subsection{Bit Error Rate (BER) Analysis}

The primary response variable used to assess impacts to the AMT link was BER. This section describes the methods used to analyze the BER response.

\subsubsection{Point and Interval Estimation}

As explained in Chapter 3, each test transmitted $3 \times 10^{8}$ bits to the AMT receiver, which reported the number of bit errors over the test interval. Assuming that bit transmissions are statistically independent, it follows that the number of bit errors for a given test follows a binomial distribution, with parameters $n$ and $p$, where $n=3 \times 10^{8}$ and the binomial proportion (or success probability), $p$, is equal to the BER. A point estimate of BER was obtained by dividing the number of reported bit errors by the total number of bits sent per test.

Because the number of reported bit errors for a test is random, the point estimate described above for BER is a random variable. One way to quantify uncertainty in the BER estimate is with a confidence interval. Confidence intervals for binomial proportions have been thoroughly studied, e.g., see [14] for a review. Methods for estimating binomial proportion confidence intervals include approximations based on a normal distribution and more exact methods based on the binomial distribution [14].

When $n=3 \times 10^{8}$, rules of thumb for the validity of the normal approximation to the binomial distribution, e.g., [15, p. 222][16], indicate that the normal approximation breaks down for BER values less than $10^{-6}$. Since the range of interest for BER in our testing was between $10^{-8}$ and $10^{-2}$, we avoided approximate methods and instead used the Clopper-Pearson confidence interval [17], which is derived directly from the discrete binomial distribution. ClopperPearson confidence intervals are known to be conservative, i.e., their coverage probability is always at least the desired nominal value [14]. In all cases, we selected the nominal coverage of the BER confidence interval to be $95 \%$.

Since the number of transmitted bits per test was $3 \times 10^{8}$, tests where the estimated BER is less than $10^{-8}$ have a large relative uncertainty (compared to the mean value). For example, if there are zero bit errors, then the $95 \%$ ClopperPearson confidence interval is $\left[0,1.2 \times 10^{-8}\right]$. This large relative uncertainty for small BER values is reflected in the log-scale plots of BER response presented later.

\subsubsection{Nonparametric Regression}

To help characterize the BER response in the main experiment, we fit a regression curve to the estimated BER as a function of the AMT signal power. Note that the purpose of the regression curve is to identify trends and that it is not intended for prediction or extrapolation. To avoid modeling assumptions, we chose to use a flexible nonparametric approach. Specifically, we used locally estimated scatterplot smoothing (LOESS) [18], a popular method for nonparametric regression [19].

Prior to performing the regression fit, the logit transformation, $\log (x /(1-x))$, was applied to the BER estimates. This transformation was used because (i) it helps to stabilize the variance of binomial proportions, i.e., makes the 
variance more uniform, and (ii) it transforms the interval $[0,1]$ to the real line [20]. After applying the LOESS method in the logit-transformed domain, the logistic transformation (the inverse logit function) was applied to transform the regression fit back to the range $[0,1]$.

To perform one-dimensional LOESS regression, we utilized a Python wrapper [21] around the original Fortran code [22]. Model parameters were specified as follows: family $=$ 'gaussian', span $=0.55$, degree $=1$, normalize $=$ False. The family parameter specifies the assumed distribution of errors. The span parameter, taking values between zero and one, controls the amount of smoothing. The degree parameter (either 1 or 2 ) is the degree of the locallyfitted polynomial. The normalize parameter determines whether the independent variables should be normalized to a common scale and is only useful for more than one dimension. For additional details, see the LOESS software user documentation [21].

Note that measurement uncertainties were not used to weight the LOESS fits. Because the BER response and its uncertainty varied across several orders of magnitude, we found that weights inversely proportional to the estimated variance of each data point led to poor fits in many circumstances. For that reason, we opted to use unweighted LOESS.

When zero bit errors were recorded for a test, the estimated BER is zero, and the logit transformation is undefined, since $\operatorname{logit}(0)=-\infty$. Moreover, as mentioned above, when the estimated BER is zero, the upper bound on the $95 \%$ Clopper-Pearson confidence interval is approximately $10^{-8}$. For these reasons, prior to fitting the regression curve, BER estimates less than $10^{-8}$ were set equal to $10^{-8}$. Empirical testing indicated that this choice had a limited impact on the regression curve, as opposed to setting zero BER estimates to a smaller value, e.g., $10^{-10}$.

\subsubsection{Plot Examples}

Here, we review plot types that are used later to present experimental results for BER. An example plot for a single whole-plot test configuration in the main experiment is shown in Figure 4.1. In this figure, BER is plotted versus AMT signal power at the receiver input, where the test results were collected on the Bedford testbed with PCM/FM at a $5 \mathrm{Mbps}$ bit rate and no $\mathrm{ABE}$. Because the test was repeated, there are separate markers for each repetition at each power level. Observe that the repeated test results agree, but that there is a missing result for Repeat 1 near $-97 \mathrm{dBm}$, due to an undetermined testbed issue.

In Figure 4.1, test cases with zero observed bit errors are denoted on the y-axis as $\nu$. Although $\nu$ appears to be located at the y-axis position corresponding to $10^{-9}$, these points actually had an estimated BER of zero (a broken y-axis was not used to simplify the plot appearance). As mentioned above, if the estimated BER is zero, the upper bound on the 95\% Clopper-Pearson confidence interval is approximately $10^{-8}$. In other words, the true BER is between zero and $10^{-8}$ with $95 \%$ confidence.

The gray boxes in Figure 4.1 indicate 90\% confidence regions for each measurement. Specifically, the vertical span of the confidence region is the 95\% Clopper-Pearson confidence interval for BER; see discussion and references in Section 4.1.1. The horizontal span of the confidence region is the $95 \%$ confidence interval for AMT signal power, modeled as a normal distribution with mean equal to the calibrated AMT signal power at the receiver input and standard deviation equal to $0.49 \mathrm{~dB}$, as specified in Appendix A, Table A.3, for the Bedford testbed. ${ }^{1}$ By the Bonferroni inequality [23, p. 13], it follows that the confidence region covers the true BER with at least $90 \%$ probability.

The black curve is the nonparametric LOESS regression fit; see Section 4.1.2. As stated above, estimated BER values of zero were set to $10^{-8}$ prior to performing the fit. This choice is reflected in the regression curve, which passes near the upper boundary of the confidence region for zero BER estimates. More generally, because the regression curve is a best fit, and not an interpolation, the curve may not perfectly follow the upper bound of the zero BER confidence

\footnotetext{
${ }^{1}$ Note that uncertainties for the Bedford and Boulder testbed are different; see Appendix A.
} 
region since it depends on other nearby measurements.

An example plot comparing the BER response from multiple ABE types in the main experiment is shown in Figure 4.2. This plot presents results collected on the Bedford testbed with PCM/FM at a $5 \mathrm{Mbps}$ bit rate. Note that the results for the no-ABE condition correspond to those given in Figure 4.1. To help make the plot easier to read, only eight of the ten ABE types are shown, with the multi-UE in-situ captures from NASA-Langley omitted. The regression fits for each $\mathrm{ABE}$ type are shown, along with markers for individual measurement results. For readability, uncertainty regions for individual measurements are not shown in this plot.

Note that the region corresponding to BER $<10^{-7}$ is shaded in Figure 4.2. This shading was added to de-emphasize BER estimates less than $10^{-7}$, because (i) they have a higher relative uncertainty, and (ii) due to their lower practical interest. However, the full range of data and their corresponding fits are shown to (i) illustrate the full extent of testing, and (ii) show all data points in cases where the observed BER values change abruptly and the fit depends strongly on BER estimates below $10^{-7}$.

As specified in the IRIG standard [1], an important performance threshold occurs at BER $=10^{-5}$. Therefore, it is useful to compare the impact of different ABE types in the main experiment with a scatterplot of the AMT signal level corresponding to $\mathrm{BER}=10^{-5}$. Figure 4.3 contains an example of such a scatterplot for the PCM/FM test results. To make this plot, the AMT RX power thresholds at $\mathrm{BER}=10^{-5}$ were estimated by inverting the respective regression fits. Note that the 5 Mbps results in Figure 4.3 correspond to the BER $=10^{-5}$ thresholds observed in Figure 4.2.

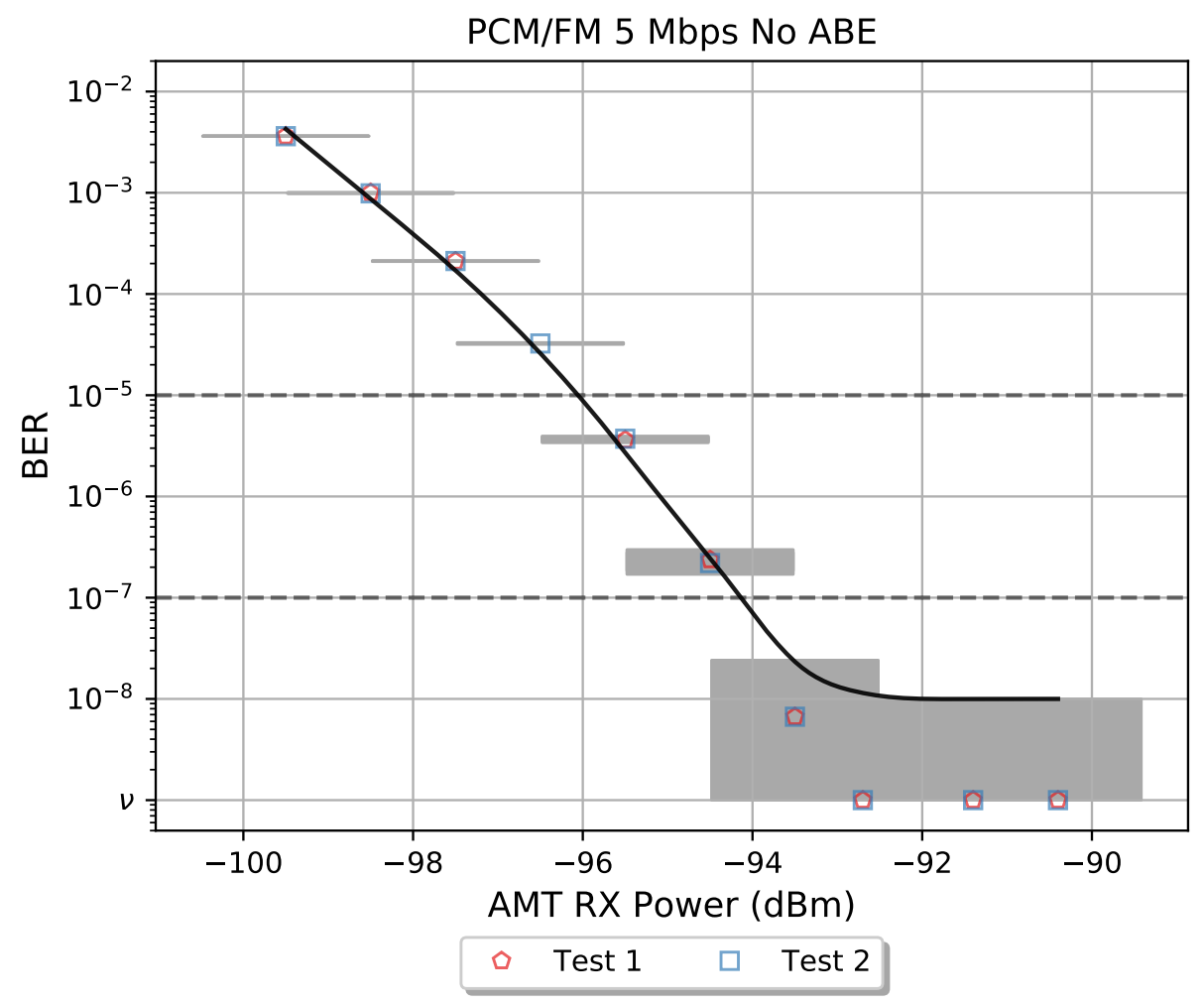

Figure 4.1: Example plot of BER vs AMT RX Power for a single whole-plot test configuration measured in Bedford. The gray boxes are $90 \%$ confidence regions for each measurement. Note that $\nu$ on the y-axis denotes test cases with zero observed bit errors. 


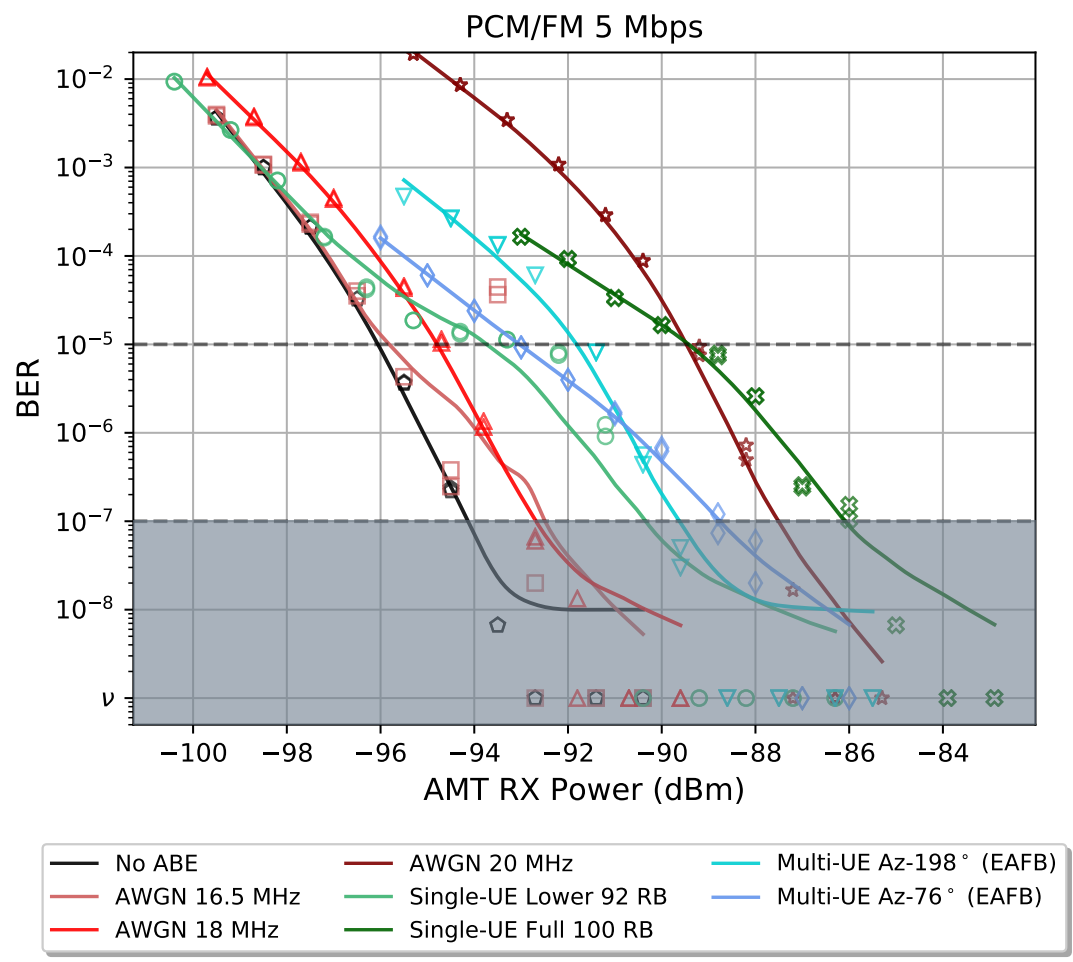

Figure 4.2: Example ABE comparison plot: BER vs RX Power. The region BER $<10^{-7}$ is shaded to indicate high relative uncertainty. Note that $\nu$ on the $\mathrm{y}$-axis denotes test cases with zero observed bit errors.

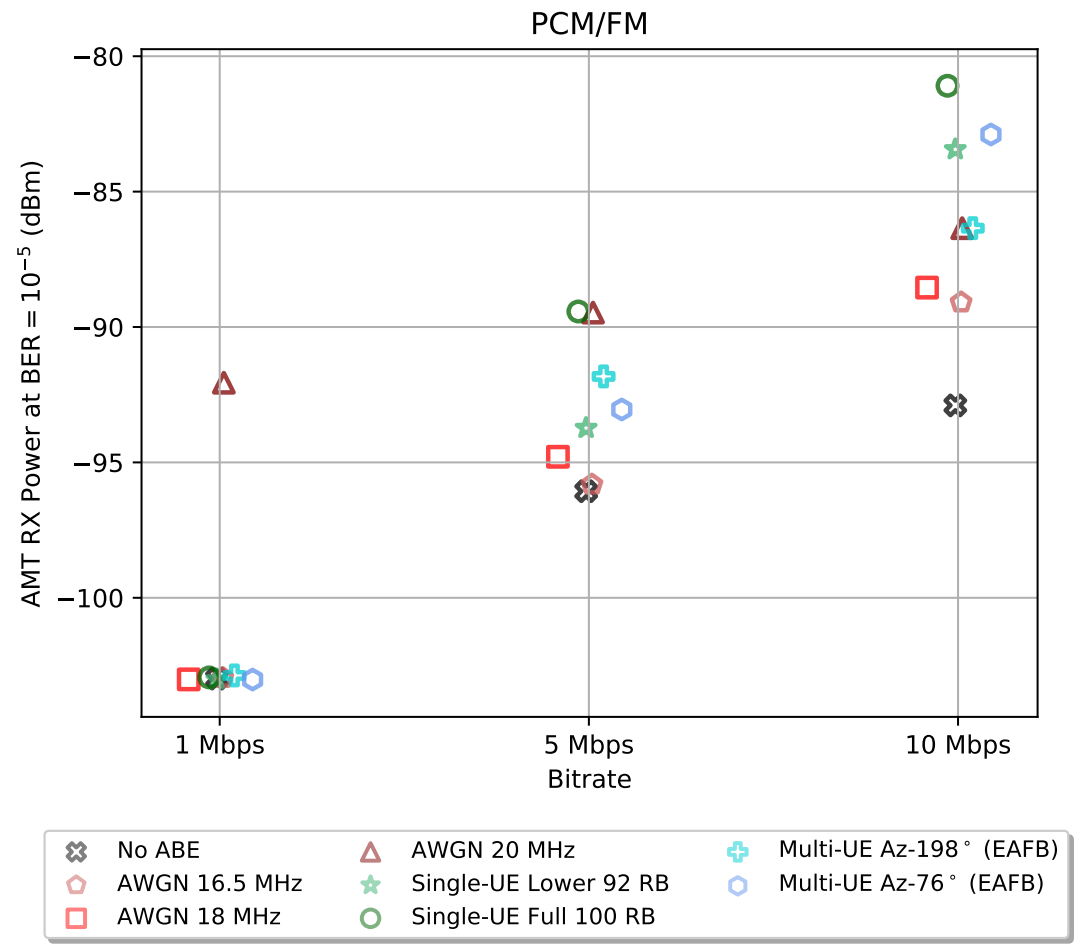

Figure 4.3: Example AMT RX power threshold plot 


\subsection{Equivalent Impact from White Ambient Noise}

\subsubsection{Definition of Effective Noise Level}

Since the impact of ambient white noise on AMT receiver performance is well-understood, it is natural to ask if it is possible to characterize the impact of adjacent band emissions on AMT performance in terms of an equivalent level of ambient white noise, i.e., a rise in the noise floor. Next, we describe an analysis method that addresses this aim.

From digital communication theory [24, 25], for an ideal receiver in an AWGN channel, BER is a monotonic function of $E_{b} / N_{0}$, the ratio of energy per bit, $E_{b}$, to noise power spectral density referred to the receiver input, $N_{0}$. Thus, at each BER value there is a corresponding $E_{b} / N_{0}$ value. We use this one-to-one relationship to define an equivalent impact from ABE.

The noise density $N_{0}$ includes contributions from the incident wave that carries ambient noise from the outside, $N_{a}$, as well as noise added inside the receiver itself. This intrinsic receiver noise is typically characterized by the noise factor, $F$ [26]. In terrestrial laboratory environments, the two are related closely with ${ }^{2}$

$$
N_{0} \approx N_{a} F
$$

Because $F$ is an unknown characteristic of the receiver under test, the characterization that follows is intended to depend only on the incident noise power density, $N_{a}$. Experimental results concerning the noise characteristics of the receiver in the Boulder testbed are given in Appendices $\mathrm{C}$ and $\mathrm{F}$, but these results are provided for completeness, and are not used to asses impact from ABE.

At a given BER level, denoted BER*, let $E_{b}^{*}$ be the corresponding energy per bit. Because this value is different without and with $\mathrm{ABE}$, we will denote it as $E_{b}^{*}$ when there are no $\mathrm{ABE}$ and $E_{b, \mathrm{ABE}}^{*}$ when $\mathrm{ABE}$ are present. For an $\mathrm{ABE}$ test case, we define the effective ambient noise power density, $N_{a}^{\text {eff }}$, such that the $E_{b}^{*} / N_{0}$ ratio is the same with and without $\mathrm{ABE}$, i.e.,

$$
\left.\frac{E_{b}^{*}}{F N_{a}}\right|_{\mathrm{BER}^{*}}=\left.\frac{E_{b, \mathrm{ABE}}^{*}}{F N_{a}^{\mathrm{eff}}}\right|_{\mathrm{BER}^{*}},
$$

taking the denominator terms from (4.1). In other words, $N_{a}^{\text {eff }}$ is defined to be the power density that yields the same BER as thermal noise at the ambient physical temperature.

We use the above definition to obtain an explicit expression for $N_{a}^{\text {eff }}$. As depicted in Figure 4.4, let $S_{n}^{*}$ and $S_{a}^{*}$ denote the signal power corresponding to $\mathrm{BER}=\mathrm{BER}^{*}$ for no- $\mathrm{ABE}$ and $\mathrm{ABE}$ cases, respectively. Also, recall that the energy per bit is equal to the signal power, $S$, divided by the bit rate, $R$, i.e., $E_{b}=S / R$. Using this fact, substituting $S_{n}^{*}$ and $S_{a}^{*}$ into equation (4.2), and simplifying, we obtain

$$
N_{a}^{\mathrm{eff}}\left(\mathrm{BER}^{*}\right)=\left.N_{a} \frac{S_{a}^{*}}{S_{n}^{*}}\right|_{\mathrm{BER}^{*}} .
$$

Thus, $N_{a}^{\text {eff }}$ is equal to the in-band noise spectral density, $N_{a}$, scaled by the ratio of signal powers at BER* in the ABE and no-ABE cases, respectively. Note that if there are no $\mathrm{ABE}$, then $S_{a}^{*}=S_{n}^{*}$ and $N_{a}^{\text {eff }}=N_{a}$. In this report, all $N_{a}^{\text {eff }}$ plots are calculated with the ambient noise level $N_{a}=-174.0 \mathrm{dBm} / \mathrm{Hz}$, corresponding to thermal noise at the standardized reference temperature, $290 \mathrm{~K} .^{3}$

\footnotetext{
${ }^{2}$ This notation may be confusing to some readers familiar with microwave noise measurement. We encapsulate all incident (and input-referred noise added inside the receiver) in $N_{0}$, following communications convention so that $E_{b} / N_{0}$ represents actual receiver SNR. This $N_{0}$ is not meant to indicate idealized blackbody noise at $290 \mathrm{~K}$.

${ }^{3}$ Analysis at other temperatures near room temperature shift $E_{b} / N_{0}$ by no more than $0.2 \mathrm{~dB}$, and only when $N_{a}^{\text {eff }}$ is within $2 \mathrm{~dB}$ of $N_{a}$
} 


\subsubsection{Examples}

\subsubsection{Excess Noise Tests}

First, using results from the excess noise experiment (side experiment C), we illustrate the consistency of the $N_{a}^{\text {eff }}$ concept for in-band noise. Recall from Section 2.5.3 that side experiment $C$ evaluated the impact of in-band excess white noise on AMT performance. In particular, tests were executed with zero, low, mid, and high excess noise power levels, $N_{R x}$.

To apply the definition of $N_{a}^{\text {eff }}$ from the previous subsection, we add excess thermal noise conditions for ABE. Figure 4.5 shows results from tests with PCM/FM modulation at a $5 \mathrm{Mbps}$ bit rate. The left plot shows the BER response for the four noise levels, and the right plot presents $N_{a}^{\text {eff }}$ for a range of BER* values. In this plot, BER* is shown on the y-axis, $N_{a}^{\text {eff }}$ is given on the top x-axis, and the equivalent $E_{b} / N_{0}$ loss relative to the baseline no excess noise condition is provided on the bottom $\mathrm{x}$-axis.

The fact that the $N_{a}^{\text {eff }}$ curves are nearly vertical indicates that the BER response curves for the excess noise conditions have roughly the same shape as the BER response curve in the baseline condition with no excess noise.

In this case, we can check the results in Figure 4.5 by predicting $N_{a}^{\text {eff }}$ from $N_{R x}$ and the receiver noise figure. Since the excess thermal noise is additive, the total effective noise density of the input-referred noise density in (4.1) is

$$
\left.N_{0}\right|_{\text {Excess noise }}=F N_{a}+N_{R x} \approx F N_{a}^{\mathrm{eff}},
$$

as long as the receiver responds to varied thermal noise as a function of $E_{b} / N_{0}$. Thus, solving for $N_{a}^{\text {eff }}$,

$$
N_{a}^{\mathrm{eff}} \approx N_{a}+\frac{N_{R x}}{F} \text { (additive thermal noise case only). }
$$

Taking $F=2.2(3.4 \mathrm{~dB})$ from Appendix F, the predicted values for $N_{a}^{\text {eff }}$ are $-168.3 \mathrm{dBm} / \mathrm{Hz}$ for $N_{R x}=-166.2 \mathrm{dBm} / \mathrm{Hz}$, $-162.6 \mathrm{dBm} / \mathrm{Hz}$ for $N_{R x}=-159.5 \mathrm{dBm} / \mathrm{Hz}$, and $-158.2 \mathrm{dBm} / \mathrm{Hz}$ for $N_{R x}=-154.9 \mathrm{dBm} / \mathrm{Hz}$. Each of these values agrees closely with the plots in Figure 4.5.

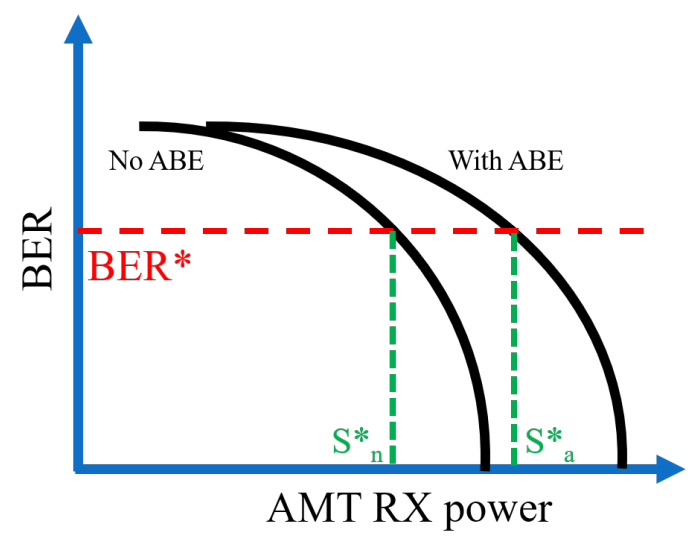

Figure 4.4: Notional plot illustrating quantities used to calculate $N_{a}^{\text {eff }}$. 

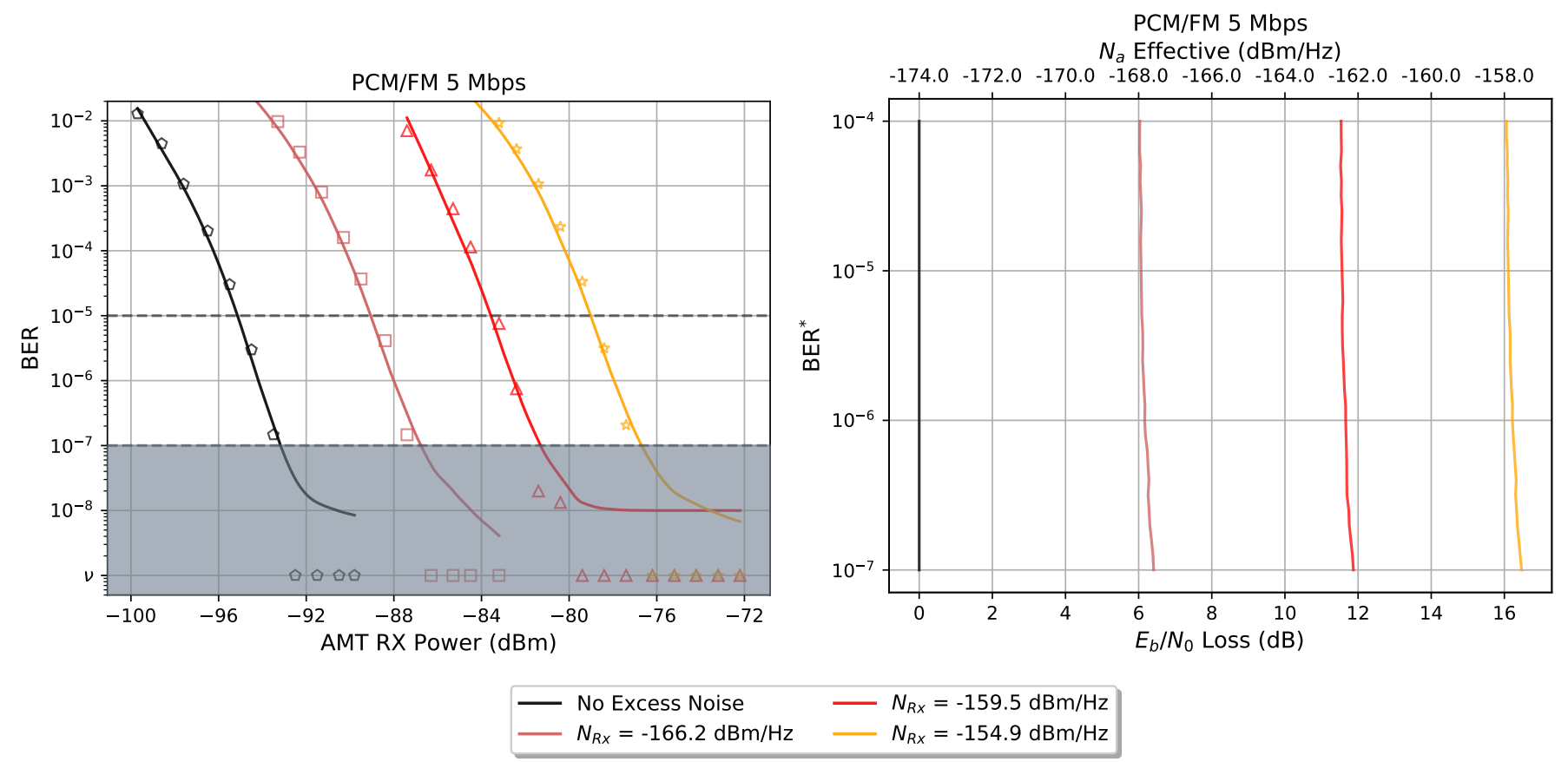

Figure 4.5: Example $N_{a}$ effective plot for an excess noise test (side experiment C). 


\subsubsection{ABE Tests}

Next, we illustrate the utility of $N_{a}^{\text {eff }}$ for ABE test conditions. Figure 4.6 shows results from ABE tests with PCM/FM modulation at a $5 \mathrm{Mbps}$ bit rate. The left plot shows the BER response for select ABE types, and the right plot presents $N_{a}^{\text {eff }}$ for a range of $\mathrm{BER}^{*}$ values. Here, $\mathrm{BER}^{*}$ is shown on the y-axis, $N_{a}^{\text {eff }}$ is given on the top x-axis, and the equivalent $E_{b} / N_{0}$ loss relative to the baseline no-ABE condition is presented on the bottom x-axis.

In the $N_{a}^{\text {eff }}$ plot, we see that for all of the LTE ABE types, the equivalent $E_{b} / N_{0}$ loss varies with BER*. For example, for the Multi-UE LTE Az- $76^{\circ} \mathrm{ABE}$ type, the equivalent $E_{b} / N_{0}$ loss ranges from $5.5 \mathrm{~dB}$ to less than $2 \mathrm{~dB}$ for BER* between $10^{-7}$ and $10^{-4}$, respectively. Hence, the equivalent impact of this ABE type changes non-uniformly with BER relative to the baseline no-ABE condition. On the other hand, two of the AWGN ABE conditions, AWGN $18 \mathrm{MHz}$ and AWGN $20 \mathrm{MHz}$, have a fairly uniform impact across different BER ${ }^{*}$ values, as evidenced by their nearly vertical $N_{a}^{\text {eff }}$ curves.
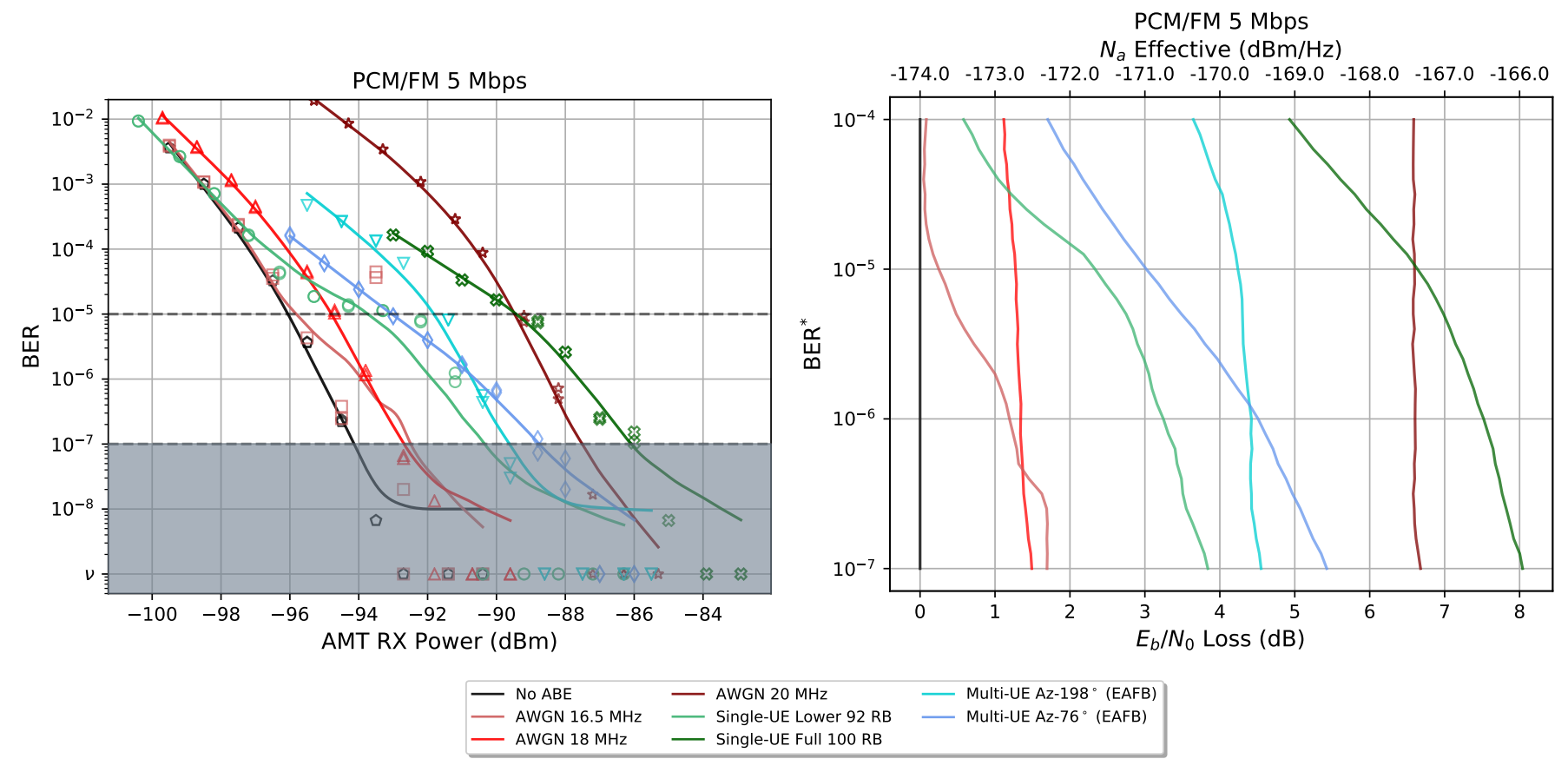

Figure 4.6: Example $N_{a}$ effective plot for ABE test conditions in the main experiment. 


\section{Chapter 5}

\section{Primary Data Analysis Results}

\subsection{Main Experiment}

This chapter presents results for the highest-priority modulation and bit rate configurations collected in Bedford (see Table 2.4). BER plots of the individual configurations from the main experiment are provided in Appendix B. Anomalous and notable results are discussed at the end of the chapter in Section 5.3. Please note that the complete set of test data for all experiments is publicly available at https:// doi.org/10.18434/M32279.

\subsubsection{ABE Power level table}

The power relationship between the ABE waveforms and the AMT signal may be described with an SABE ratio. Typically this definition assumes a ratio of root mean square (RMS) power of the two non co-located but adjacent bands. However, the comparison of SNR type of quantities in adjacent band susceptibility studies are incomplete without considering both peak and RMS power values. Thus we identify, where applicable, the peak, or RMS value. Note that some power representations, such as the peak power of AWGN waveforms, are not meaningful.

Table 5.1 lists the peak and average power of the ABE waveforms used in the testbeds to generate the results in Section 5.1.2. The AMT average power levels are swept across a range of values as depicted in the $x$-axis in the BER versus AMT RX power. For example, see the left-side plot in Figure 5.1.

The calculated values refer to the ABE and AMT power levels at the plane of the AMT RX. The process for calculating these values are outlined in Section A.2 and Section A.1.

\subsubsection{ABE Comparison Plots}

The ABE comparison plots, Figures 5.1 through 5.7, show results of BER versus AMT RX power (left-side plots) and the BER* versus $N_{a}^{\text {eff }}$ and $E_{b} / N_{0}$ Loss (right-side plots). The primary region of interest lies between BER values of $10^{-7}$ and $10^{-4}$, which is also the range of the $y$-axis on the BER* plots. The black curve represents the baseline conditions where no ABE is present. For the plots in this section, the multi-UE cases of "Multi-UE-LTE-az-198" and

Table 5.1: ABE Power level table, Main Experiment campaign conducted at the MITRE Bedford, MA testbed.

\begin{tabular}{|l|l|l|}
\hline Waveform Name & Average Power & Peak Power \\
\hline AWGN 20 MHz & $-75.8 \mathrm{dBm} \pm 0.9 \mathrm{~dB}$ & N/A \\
AWGN 18 MHz & $-76.4 \mathrm{dBm} \pm 0.9 \mathrm{~dB}$ & N/A \\
AWGN 16.5 MHz & $-76.8 \mathrm{dBm} \pm 0.9 \mathrm{~dB}$ & N/A \\
Single-UE Full 100 RB (EAFB, 20 MHz Filter) & $-69.9 \mathrm{dBm} \pm 1.1 \mathrm{~dB}$ & $-61.8 \mathrm{dBm} \pm 1.1 \mathrm{~dB}$ \\
Single-UE Lower 92 RB (EAFB, 20 MHz Filter) & $-72.8 \mathrm{dBm} \pm 1.1 \mathrm{~dB}$ & $-62.3 \mathrm{dBm} \pm 1.1 \mathrm{~dB}$ \\
Multi-UE Az-76 $100 \mathrm{RB}$ (EAFB, 20 MHz Filter) & $-84.6 \mathrm{dBm} \pm 1.1 \mathrm{~dB}$ & $-62.1 \mathrm{dBm} \pm 1.1 \mathrm{~dB}$ \\
Multi-UE Az-198 $100 \mathrm{RB}$ (EAFB, 20 MHz Filter) & $-86.5 \mathrm{dBm} \pm 1.1 \mathrm{~dB}$ & $-62.1 \mathrm{dBm} \pm 1.1 \mathrm{~dB}$ \\
Multi-UE Az-140 $100 \mathrm{RB}$ (LARC, 20 MHz Filter) & $-80.3 \mathrm{dBm} \pm 1.1 \mathrm{~dB}$ & $-62.1 \mathrm{dBm} \pm 1.1 \mathrm{~dB}$ \\
Mult-UE Az-165 $100 \mathrm{RB}$ (LARC, 20 MHz Filter) & $-78.9 \mathrm{dBm} \pm 1.1 \mathrm{~dB}$ & $-62.1 \mathrm{dBm} \pm 1.1 \mathrm{~dB}$ \\
\hline
\end{tabular}


"Multi-UE-LTE-az-76" are shown as those waveforms are in-situ captures from the EAFB telemetry site.

Figures 5.1 through 5.7 show that the AWGN ABE waveforms produce a response in the expected manner, with the 16.5 MHz AWGN waveform producing the least impact and the $20 \mathrm{MHz}$ AWGN yielding the most impact. This is evident by the amount of AMT RX power required to maintain the same BER. Observe that the $N_{a}^{\text {eff }}$ plots are generally vertical for the AWGN ABE types, indicating a uniform impact relative to the baseline no-ABE condition.

The responses due to the other ABE waveforms varies. Figure 5.1 shows very little impact for the case of PCM/FM modulation at $1 \mathrm{Mbps}$. However, at $5 \mathrm{Mbps}$, differences start occurring. One general trend is that the "Single-UE-LTE Full" waveform causes the largest impact. This could be attributed to the higher average power, but this would not completely explain the differences since the two multi-UE cases both cause more impact than the "Single-UE-LTE 92RB" yet have average powers that are at least $10 \mathrm{~dB}$ below the "Single-UE-LTE 92RB" waveform.

Another behavior of note are the rapid transitions from $10^{-7}$ to $10^{-4}$ shown in the FEC cases, Figure 5.5 and Figure 5.6, for the AWGN waveforms. In contrast, except for the for the SOQPSK $5 \mathrm{Mbps}$ result, the single and multi-UE show a slower, non-linear transition. In addition, there is a noticeable separation on the AMT RX axis between the AWGN and the single and multi-UE curves. These factors are an indication that the FEC algorithm does not work as well to correct errors caused by non-AWGN noise or interference.
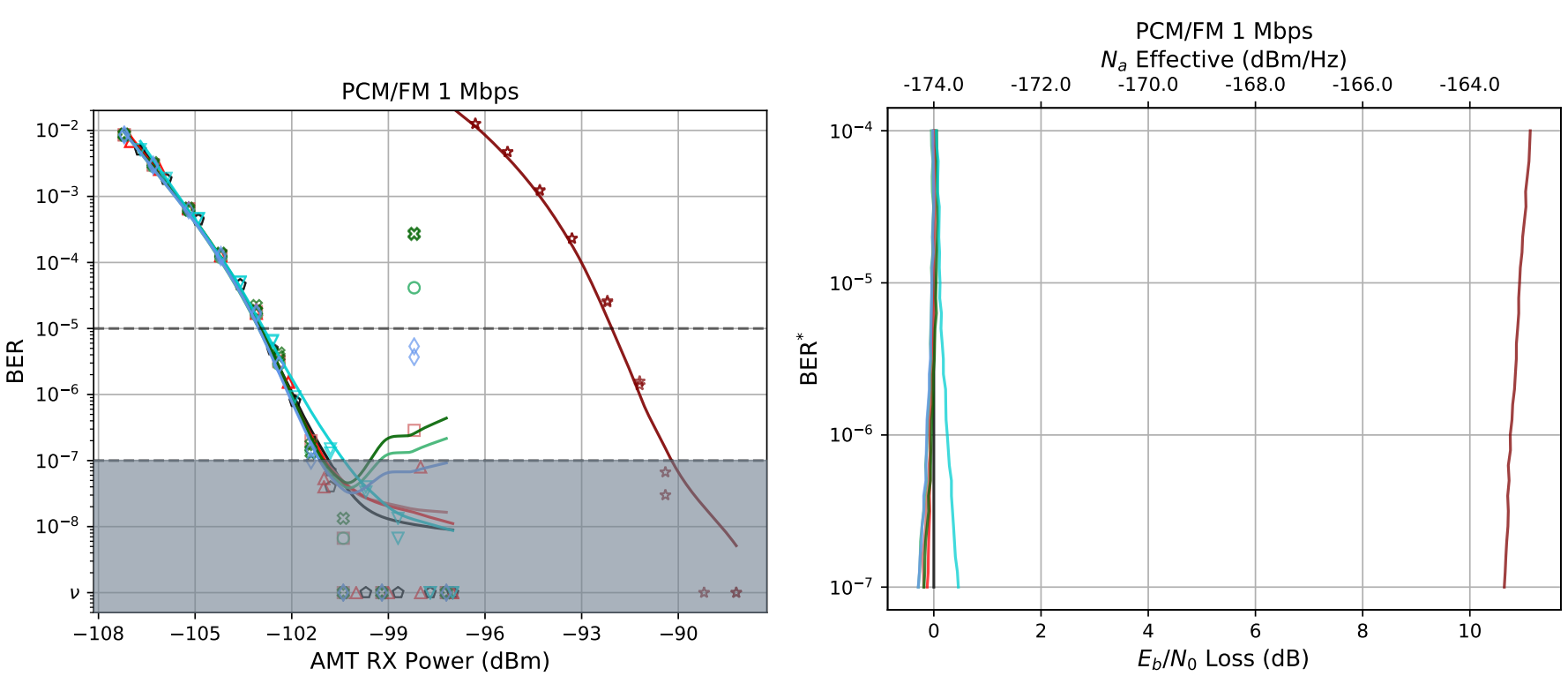

\begin{tabular}{lll} 
- No ABE & AWGN $20 \mathrm{MHz}$ & - Multi-UE Az-198 \\
\hline AWGN $16.5 \mathrm{MHz}$ & - Single-UE Lower 92 RB \\
AWGN 18 MHz & - Single-UE Full 100 RB & \\
\hline
\end{tabular}

Figure 5.1: BER vs AMT RX power and BER* vs $N_{a}$ Effective for selected ABE types: PCM/FM 1 Mbps. The region $\mathrm{BER}<10^{-7}$ is shaded in the left plot to indicate high relative uncertainty. The anomalous responses at $-98 \mathrm{dBm}$ in the left plot are discussed in Section 5.3. 

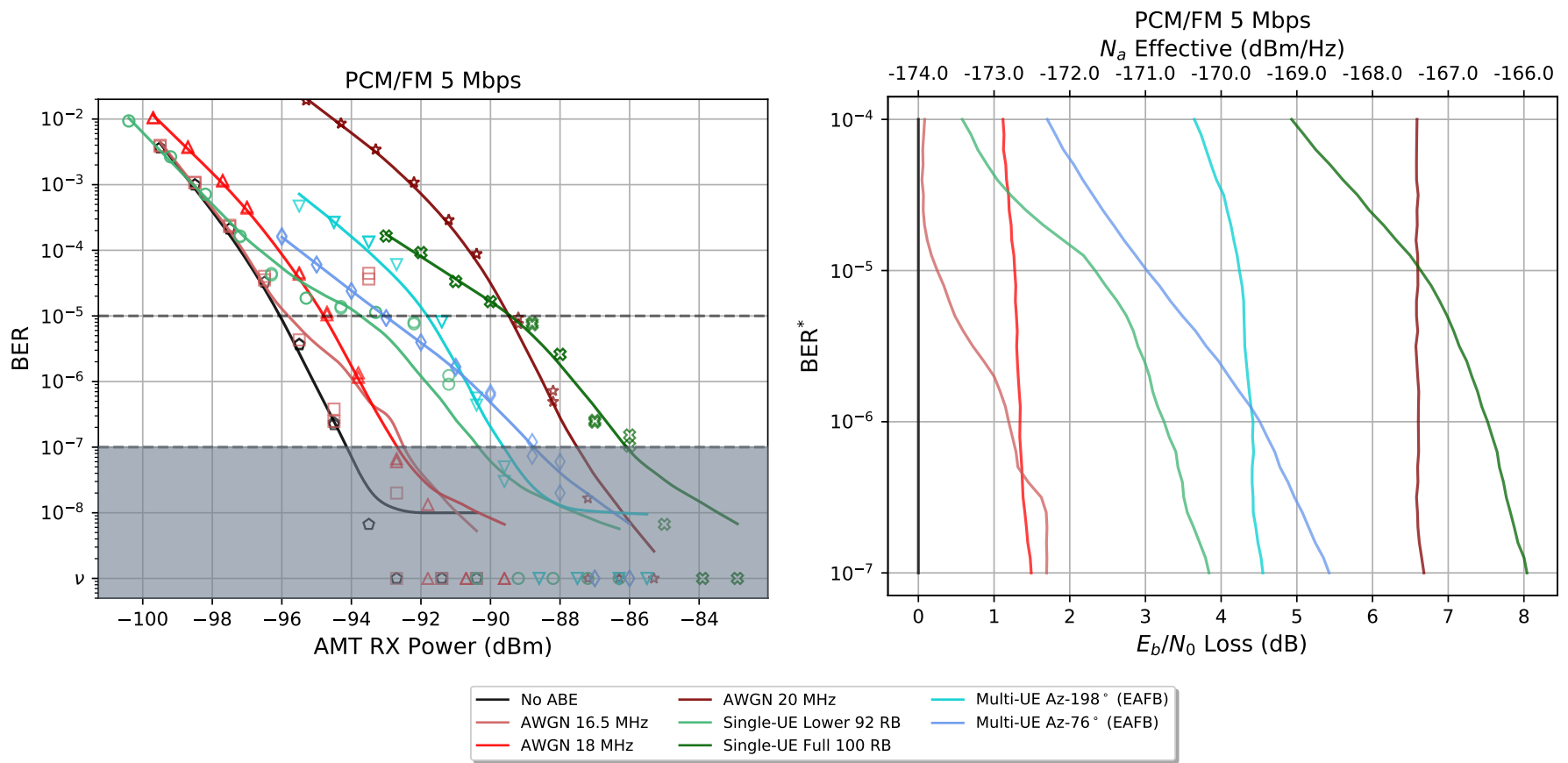

Figure 5.2: BER vs AMT RX power and BER* vs $N_{a}$ Effective for selected ABE types: PCM/FM 5 Mbps. The region $\mathrm{BER}<10^{-7}$ is shaded in the left plot to indicate high relative uncertainty.

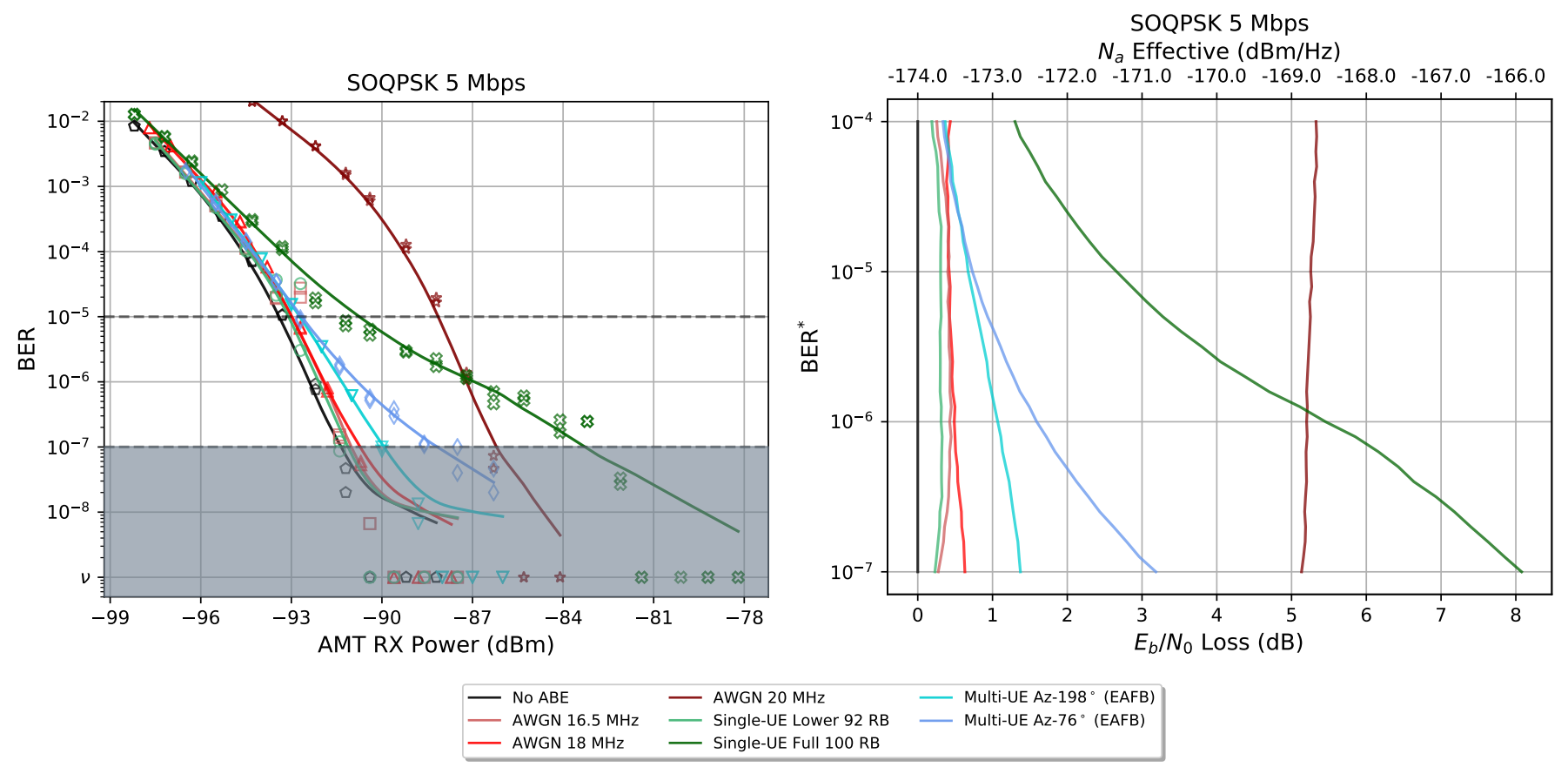

Figure 5.3: BER vs AMT RX power and BER* vs $N_{a}$ Effective for selected ABE types: SOQPSK 5 Mbps. The region BER $<10^{-7}$ is shaded in the left plot to indicate high relative uncertainty. 

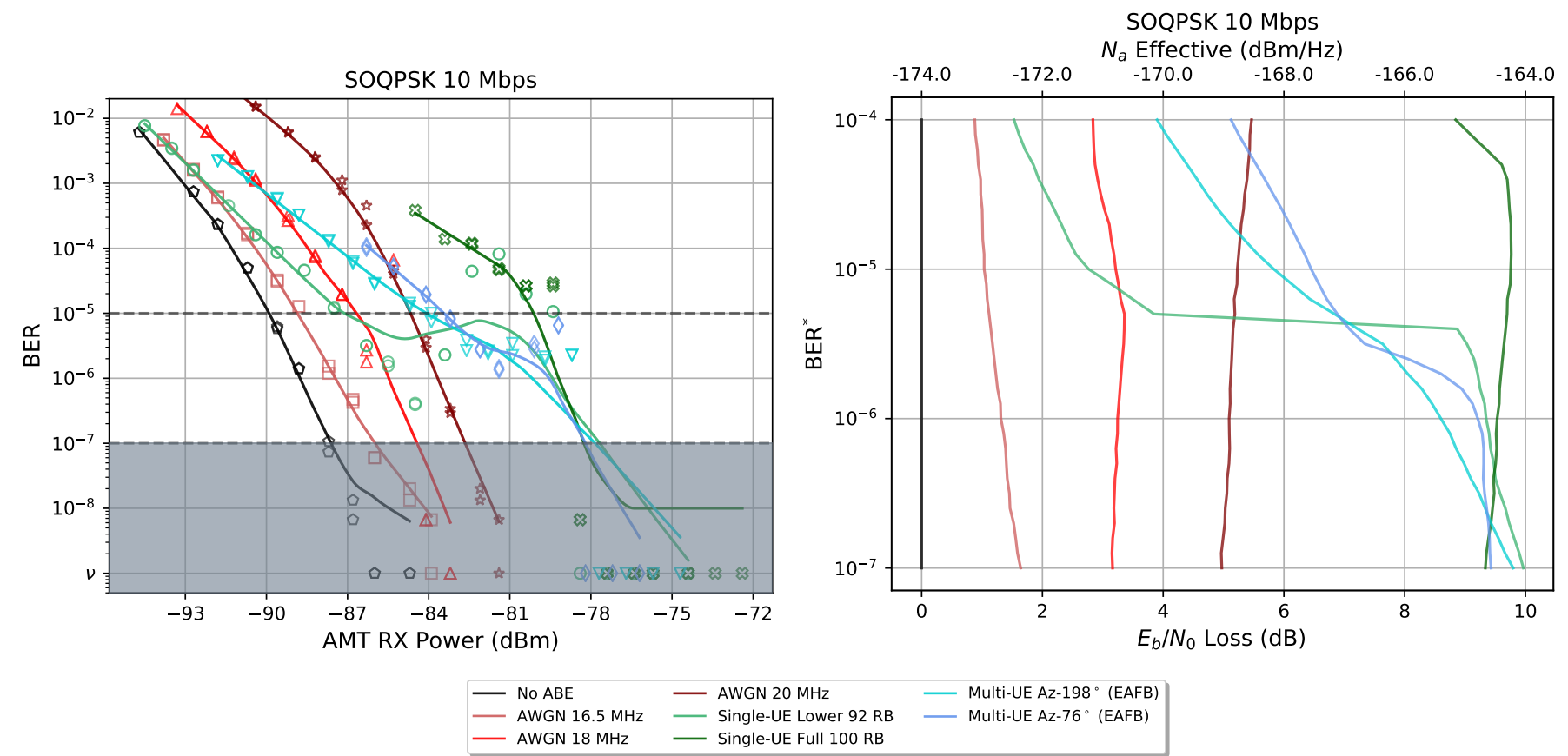

Figure 5.4: BER vs AMT RX power and BER* vs $N_{a}$ Effective for selected ABE types: SOQPSK 10 Mbps. The region $\mathrm{BER}<10^{-7}$ is shaded in the left plot to indicate high relative uncertainty.

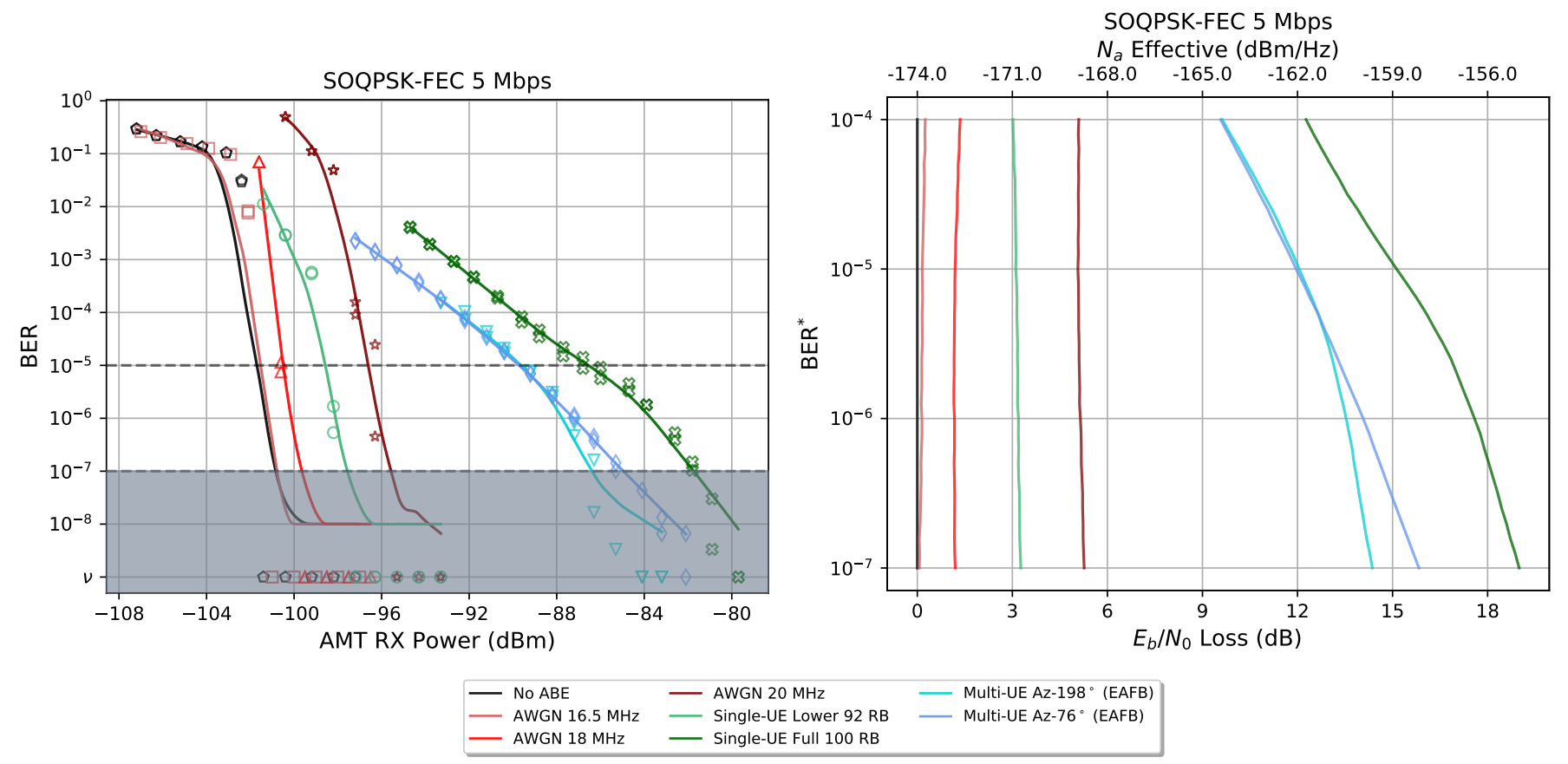

Figure 5.5: BER vs AMT RX power and BER* vs $N_{a}$ Effective for selected ABE types: SOQPSK-FEC 5 Mbps. The region BER $<10^{-7}$ is shaded in the left plot to indicate high relative uncertainty. 


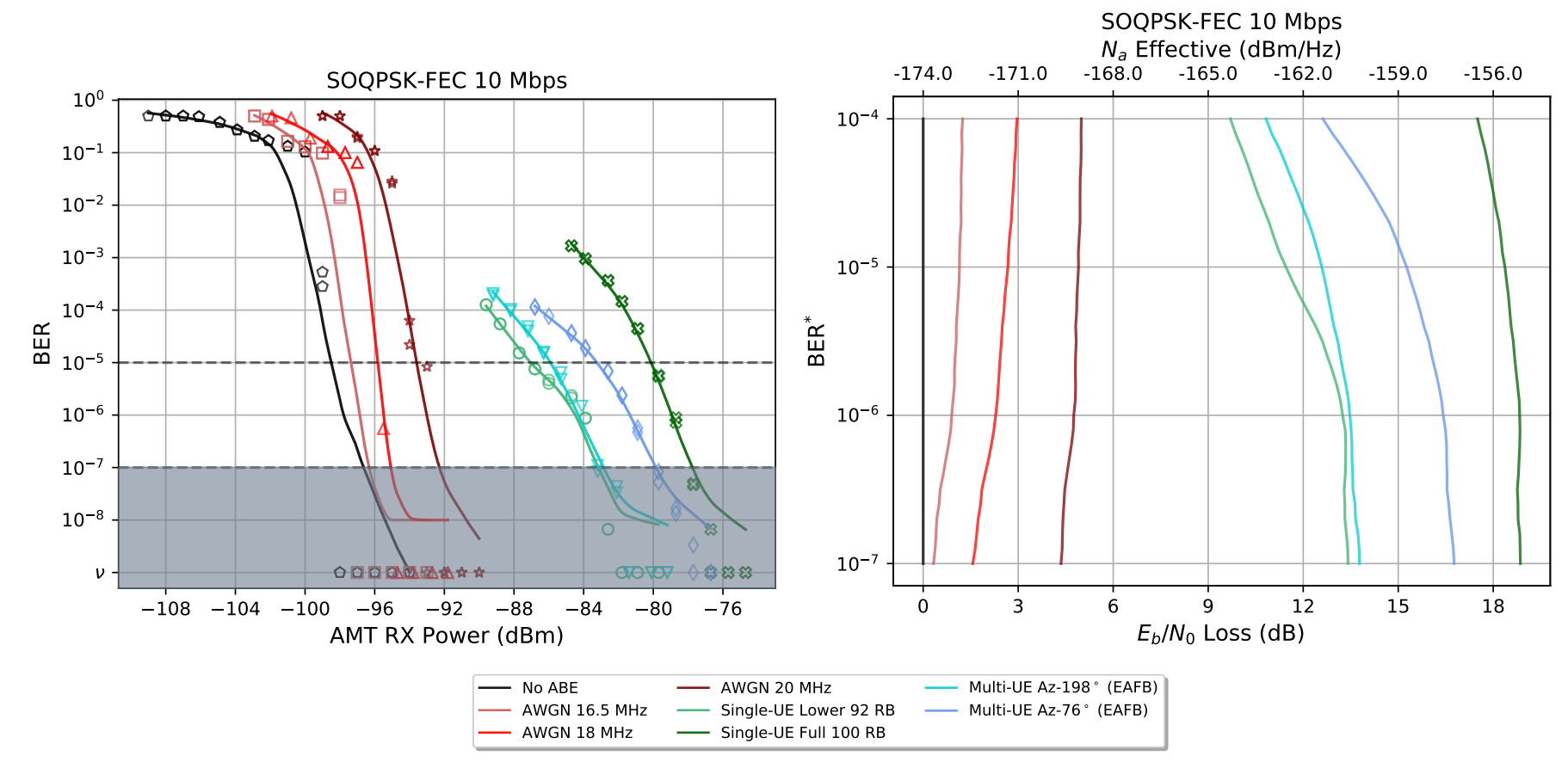

Figure 5.6: BER vs AMT RX power and BER* vs $N_{a}$ Effective for selected ABE types: SOQPSK-FEC 10 Mbps. The region $\mathrm{BER}<10^{-7}$ is shaded in the left plot to indicate high relative uncertainty.

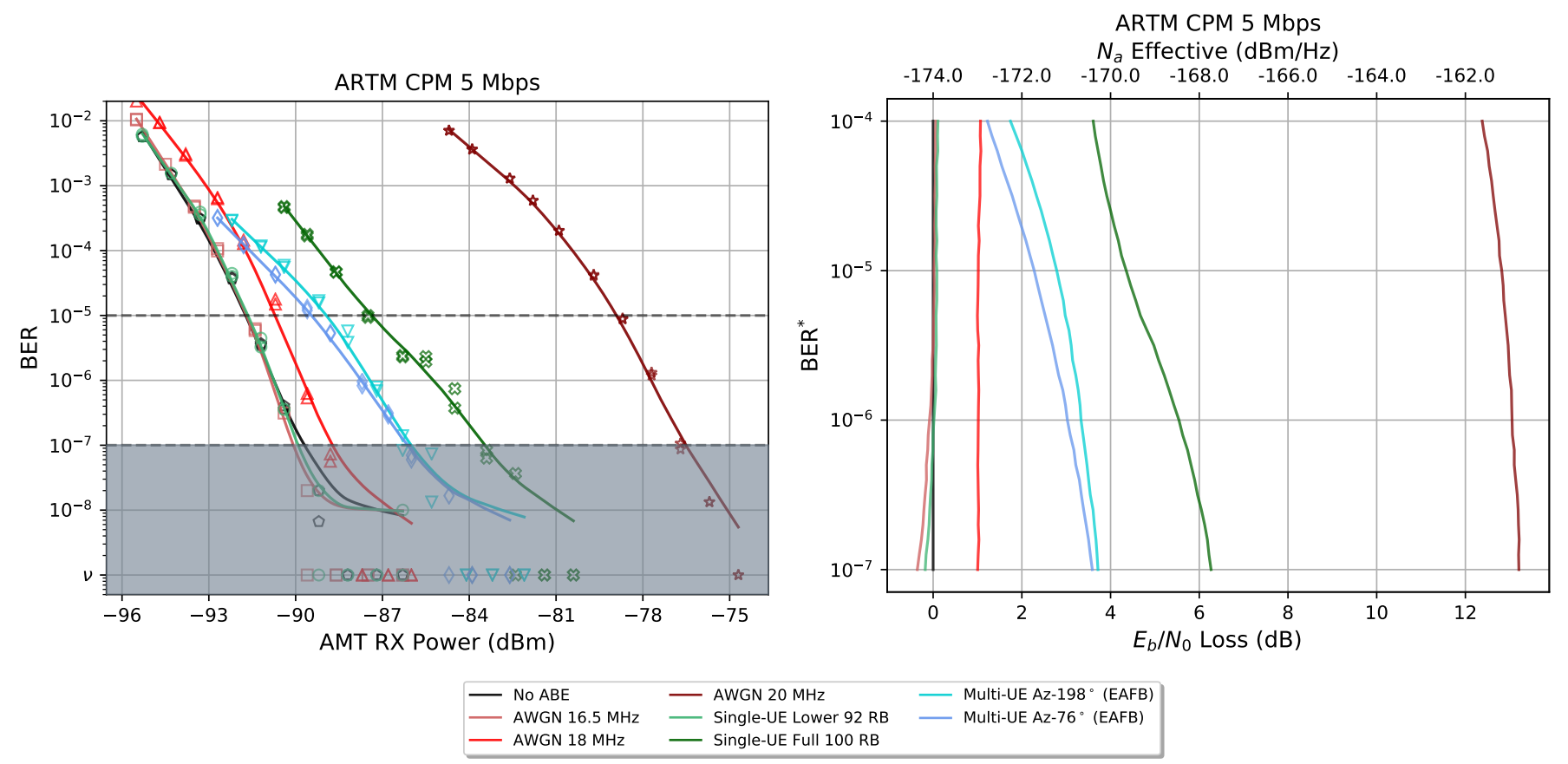

Figure 5.7: BER vs AMT RX power and BER* vs $N_{a}$ Effective for selected ABE types: ARTM CPM 5 Mbps. The region $\mathrm{BER}<10^{-7}$ is shaded in the left plot to indicate high relative uncertainty. 


\subsubsection{RX Power Threshold Plots}

An AMT link with a BER less than $10^{-5}$ is considered an acceptable link. Figure 5.8 shows the AMT RX power that result in a BER $=10^{-5}$ based on the results in 5.1.2, with some additional bit rates, e.g., 20 Mbps, results included. As expected, the general trend is that increasing bit rates require increasing AMT RX power to maintain a BER $=10^{-5}$. Though not pursued here, additional analysis on the slope of the increases might provide additional insight of the impact differences due to $\mathrm{ABE}$ type.
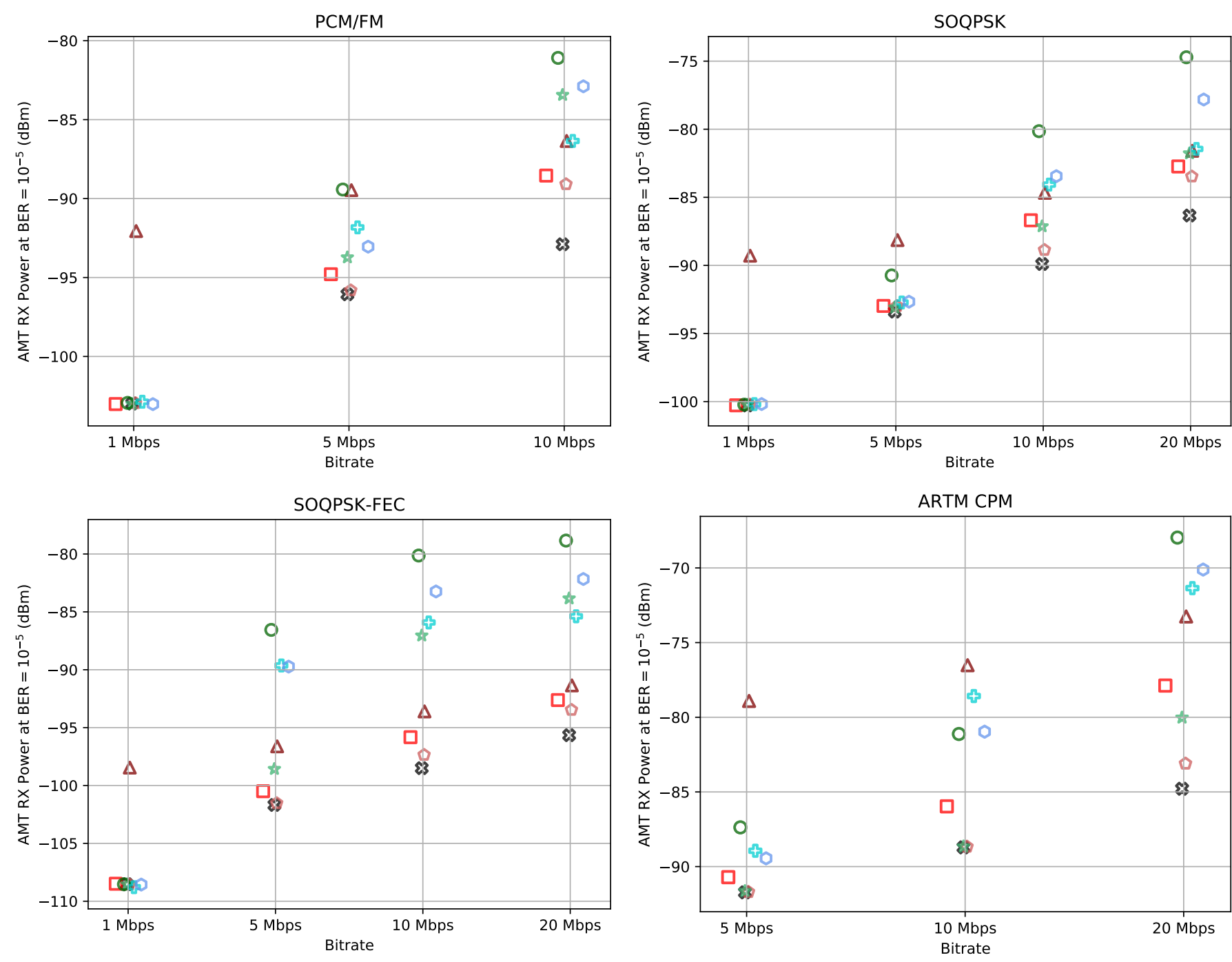

\begin{tabular}{|c|c|c|c|c|c|}
\hline 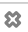 & No $A B E$ & $\Delta$ & AWGN $20 \mathrm{MHz}$ & के & Multi-UE Az-198 ${ }^{\circ}$ (EAFB) \\
\hline 0 & AWGN $16.5 \mathrm{MHz}$ & * & Single-UE Lower 92 RB & 0 & Multi-UE Az-76 (EAFB) \\
\hline$\square$ & AWGN $18 \mathrm{MHz}$ & 0 & Single-UE Full 100 RB & & \\
\hline
\end{tabular}

Figure 5.8: Scatter plots of AMT RX Power at corresponding to a BER of $10^{-5}$ vs AMT modulation and bitrate 


\subsubsection{No-ABE Comparison plots}
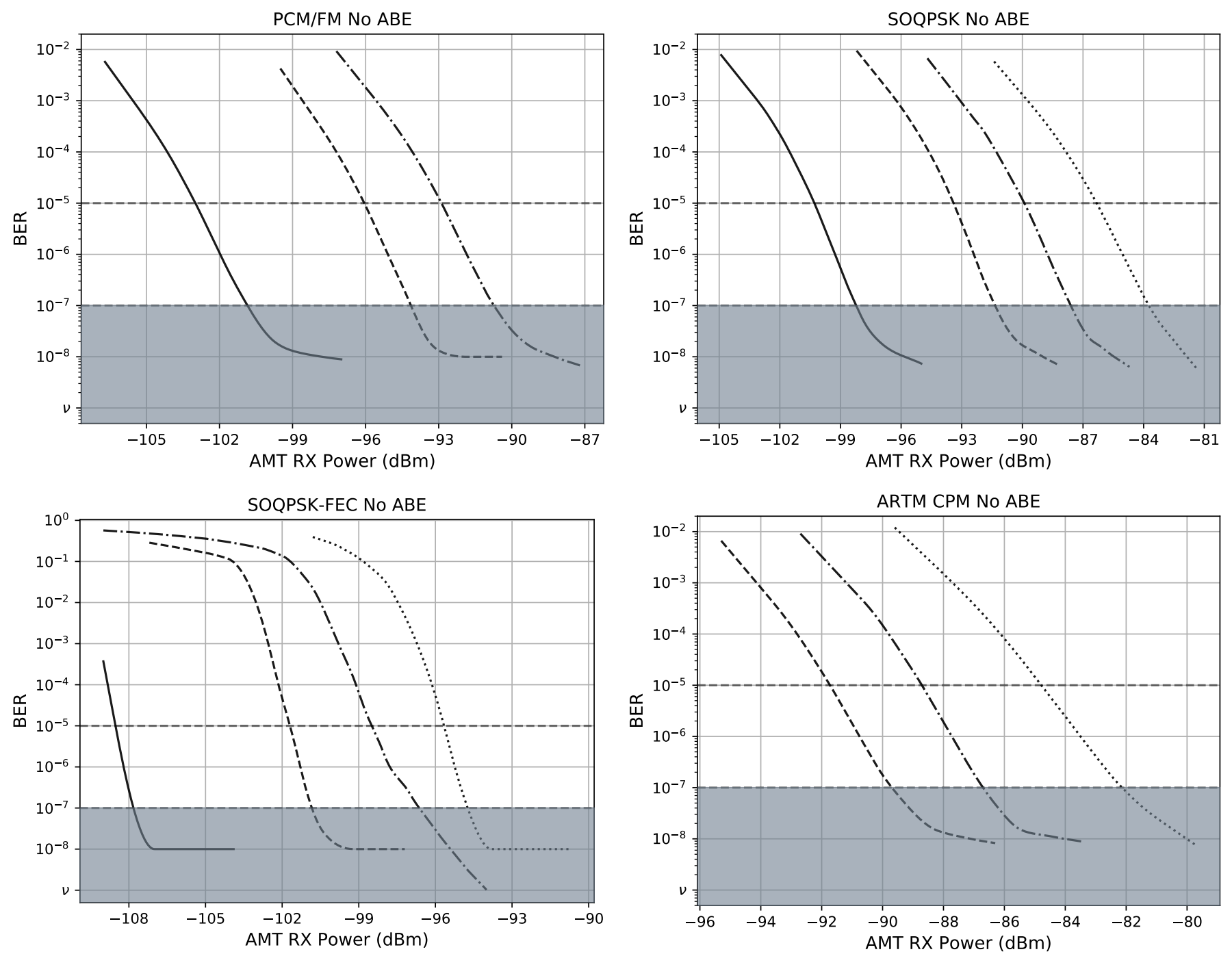

\begin{tabular}{rrrr}
\hline & $1 \mathrm{Mbps}$ & $-\cdot-$ & $10 \mathrm{Mbps}$ \\
--- & $5 \mathrm{Mbps}$ & $\ldots$. & $20 \mathrm{Mbps}$ \\
\hline
\end{tabular}

Figure 5.9: BER vs AMT RX power for No-ABE tests. The region BER $<10^{-7}$ is shaded to indicate high relative uncertainty. 
Table 5.2: ABE Power level table, for the Frequency Offset Experiment as conducted at MITRE Bedford, MA

\begin{tabular}{|l|l|l|}
\hline Waveform Name & Average Power & Peak Power \\
\hline AWGN 20 MHz & $-59.6 \mathrm{dBm} \pm 0.9 \mathrm{~dB}$ & N/A \\
Single-UE Full 100 RB (EAFB, 20 MHz Filter) & $-60.5 \mathrm{dBm} \pm 1.1 \mathrm{~dB}$ & $-52.2 \mathrm{dBm} \pm 1.1 \mathrm{~dB}$ \\
Single-UE Lower 92 RB (EAFB, 20 MHz Filter) & $-63.5 \mathrm{dBm} \pm 1.1 \mathrm{~dB}$ & $-52.7 \mathrm{dBm} \pm 1.1 \mathrm{~dB}$ \\
Single-UE Full 100 RB (EAFB, 37 MHz Filter) & $-60.5 \mathrm{dBm} \pm 1.1 \mathrm{~dB}$ & $-52.4 \mathrm{dBm} \pm 1.1 \mathrm{~dB}$ \\
Single-UE Lower 92 RB (EAFB, 37 MHz Filter) & $-63.4 \mathrm{dBm} \pm 1.1 \mathrm{~dB}$ & $-52.8 \mathrm{dBm} \pm 1.1 \mathrm{~dB}$ \\
Multi-UE Az-76 $100 \mathrm{RB}$ (EAFB, 37 MHz Filter) & $-70.6 \mathrm{dBm} \pm 1.1 \mathrm{~dB}$ & $-48.0 \mathrm{dBm} \pm 1.1 \mathrm{~dB}$ \\
Multi-UE Az-198 $100 \mathrm{RB}$ (EAFB, 37 MHz Filter) & $-82.5 \mathrm{dBm} \pm 1.1 \mathrm{~dB}$ & $-53.9 \mathrm{dBm} \pm 1.1 \mathrm{~dB}$ \\
Single-UE Full 100 RB (Lab, 49 MHz Filter) & $-66.0 \mathrm{dBm} \pm 1.1 \mathrm{~dB}$ & $-52.9 \mathrm{dBm} \pm 1.1 \mathrm{~dB}$ \\
Single-UE Lower 90 RB (Lab, 49 MHz Filter) & $-65.4 \mathrm{dBm} \pm 1.1 \mathrm{~dB}$ & $-52.9 \mathrm{dBm} \pm 1.1 \mathrm{~dB}$ \\
Single-UE Full 25 RB (Lab, 49 MHz Filter) & $-70.7 \mathrm{dBm} \pm 1.1 \mathrm{~dB}$ & $-52.9 \mathrm{dBm} \pm 1.1 \mathrm{~dB}$ \\
\hline
\end{tabular}

\subsection{Frequency Offset Experiment}

The frequency offset experiments are designed to investigate if the band edge back-off frequency calculation specified in [1] provides sufficient placement of the AMT signal with respect to the ABE. The goal is to provide assurance that the ABE will not impact the AMT link, balanced with utilizing as much of the allocated AMT band as possible.

\subsubsection{ABE Power level table}

Table 5.2 contains the RMS average and peak powers for the ABE waveforms tested here. Note this subset of waveforms focuses on EAFB conditions and several in-situ and laboratory LTE RB blanking conditions.

\subsubsection{Frequency Offset Results}

Figures 5.10 through 5.16 show results based on Table 5.2 ABE power levels and filtering. These power levels are set to match the peak power of the in-situ captures. The black, dashed vertical line represents the center frequency of the AMT signal when $1780 \mathrm{MHz}$ is the lowest frequency of the $99 \%$ occupied bandwidth. The red, dashed vertical line represents the AMT center frequency as calculated to meet the IRIG recommendations for a $10 \mathrm{~W}$ transmitter operating and a $1780 \mathrm{MHz}$ band edge.

In order to meet the IRIG recommendations, the curve due to the presence of ABE should be below a BER value of $10^{-5}$ when crossing the red, dashed vertical line. Alternatively, the distance between where the curves cross the $\mathrm{BER}=10^{-5}$ point and the red, dashed vertical line implies the frequency margin of the IRIG recommendations when ignoring the AMT transmitter's emission profile. Note that the IRIG recommended lowest frequency is based on AMT transmitter performance.

The observed results show that there is a distinct change in receiver behavior when subjected to ABE waveforms that are comprised of a single UE versus the multi-UE, and again when the waveform was derived from laboratory or in-situ recordings, Figures 5.10-5.16 highlight the laboratory waveform results on the left, and the in-situ waveform results on the right.

In-situ multi-UE waveforms exhibit a more frequency persistent response (i.e., slow convergence to the $\mathrm{BER}=10^{-8}$ level) as compared to the single-UE recordings. With the single-UE waveforms, the in-situ recordings elicit a more persistent response as compared to the laboratory captures.

In terms of AMT modulation formats, the receiver response is traced to the bit rate and its implicit relation with receiver IF bandwidth. Most notably however, FEC has a significant impact in altering the receiver behavior as compared to the non-FEC counterparts. Furthermore, ARTM CPM exhibits a similar response as the SOQPSK modulation, however, the IRIG recommended center frequency is shifted compared to the SOQPSK case. 
Finally, the convergence below a BER value of $10^{-5}$ is slower than anticipated for several combinations of multiUE waveforms with different AMT modulation formats, e.g., mutli-UE-LTE az- $198^{\circ}$ and SOQPSK 5 Mbps. This non-intuitive behavior is discussed in Section 5.3.
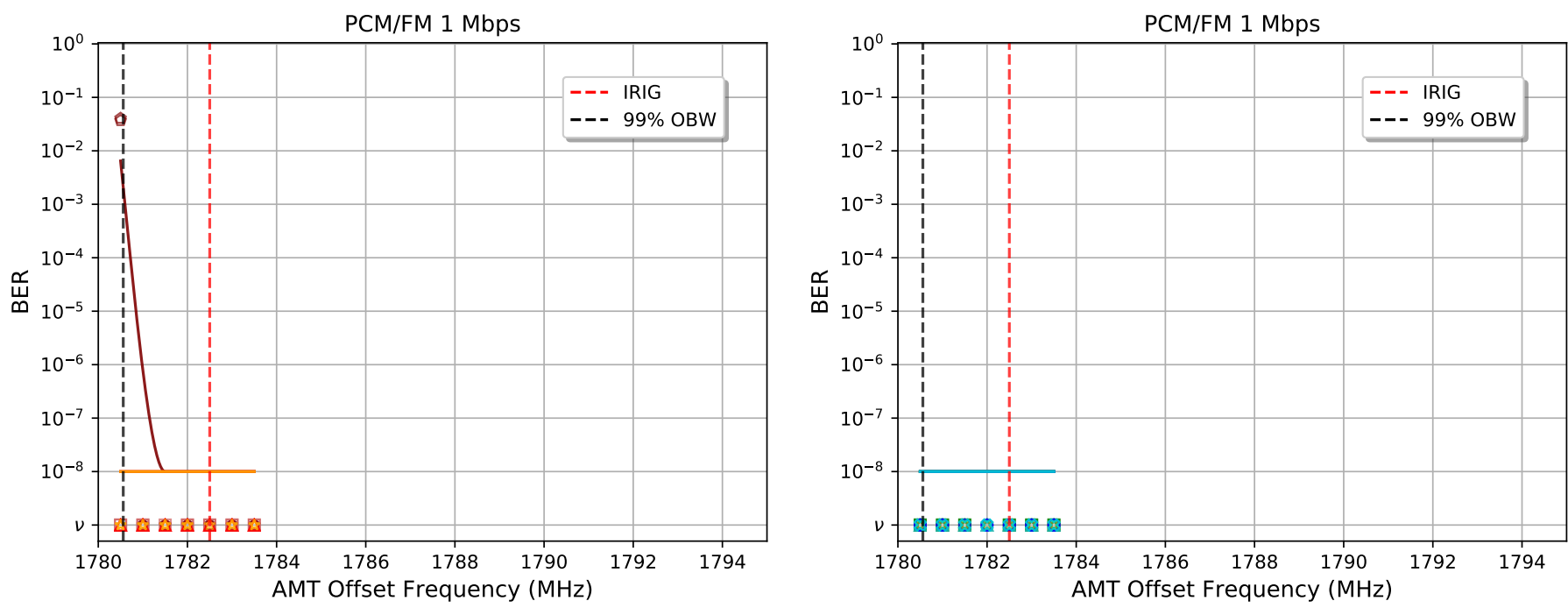

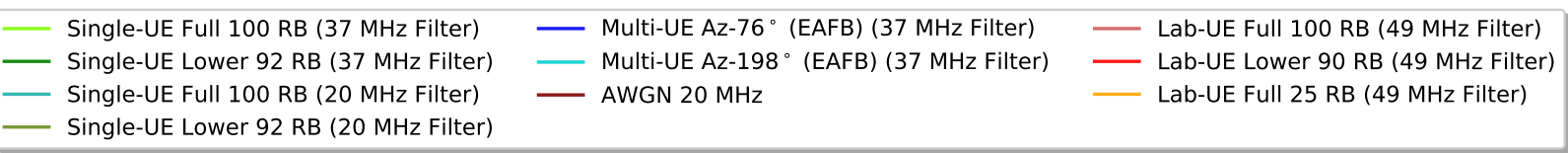

Figure 5.10: BER vs AMT Offset Frequency for all ABE types: PCM/FM 1 Mbps, (left) response due to laboratory ABE types, (right) due to in-situ ABE types. Note that points and curves for different ABE types may be identical or nearly identical, which makes the results on the plots difficult to distinguish. 

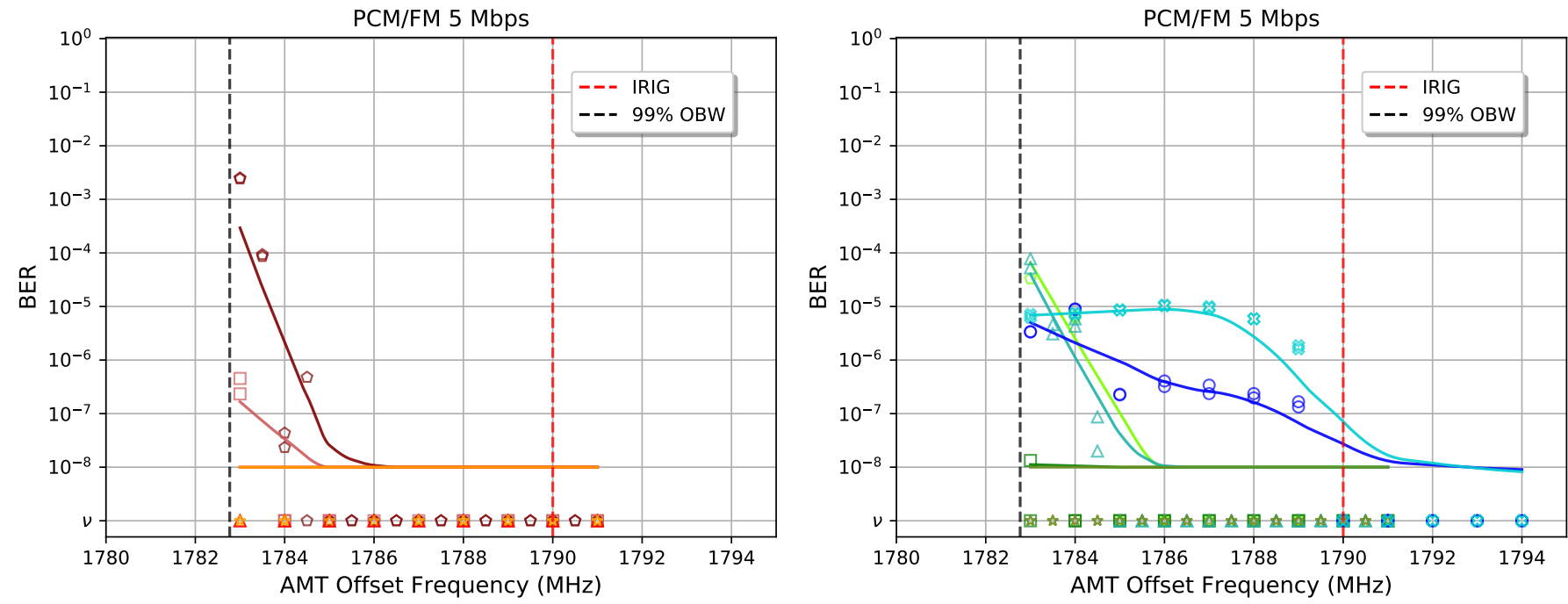

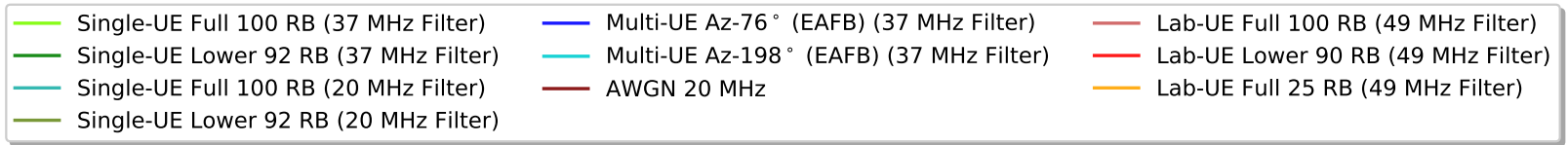

Figure 5.11: BER vs AMT Offset Frequency for all ABE types: PCM/FM 5 Mbps, (left) response due to laboratory ABE types, (right) due to in-situ ABE types.
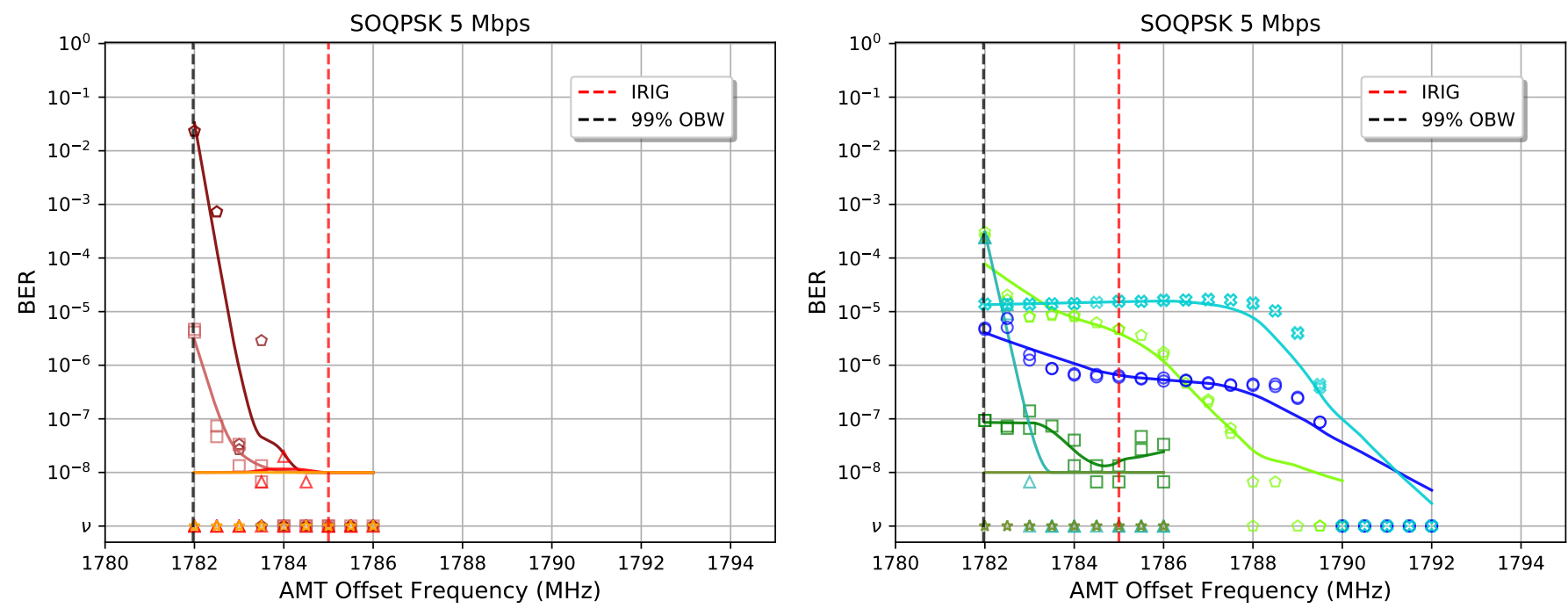

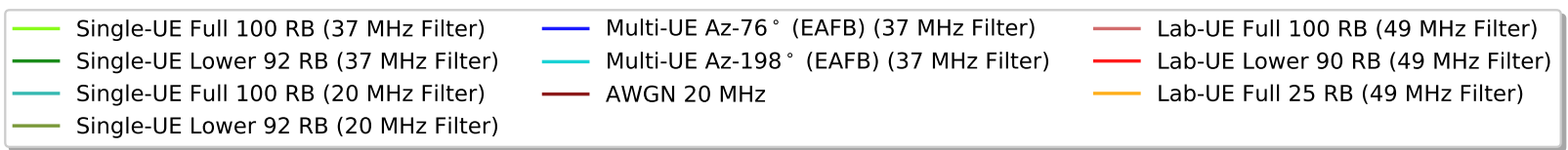

Figure 5.12: BER vs AMT Offset Frequency for all ABE types: SOQPSK 5 Mbps, (left) response due to laboratory $\mathrm{ABE}$ types, (right) due to in-situ $\mathrm{ABE}$ types. 

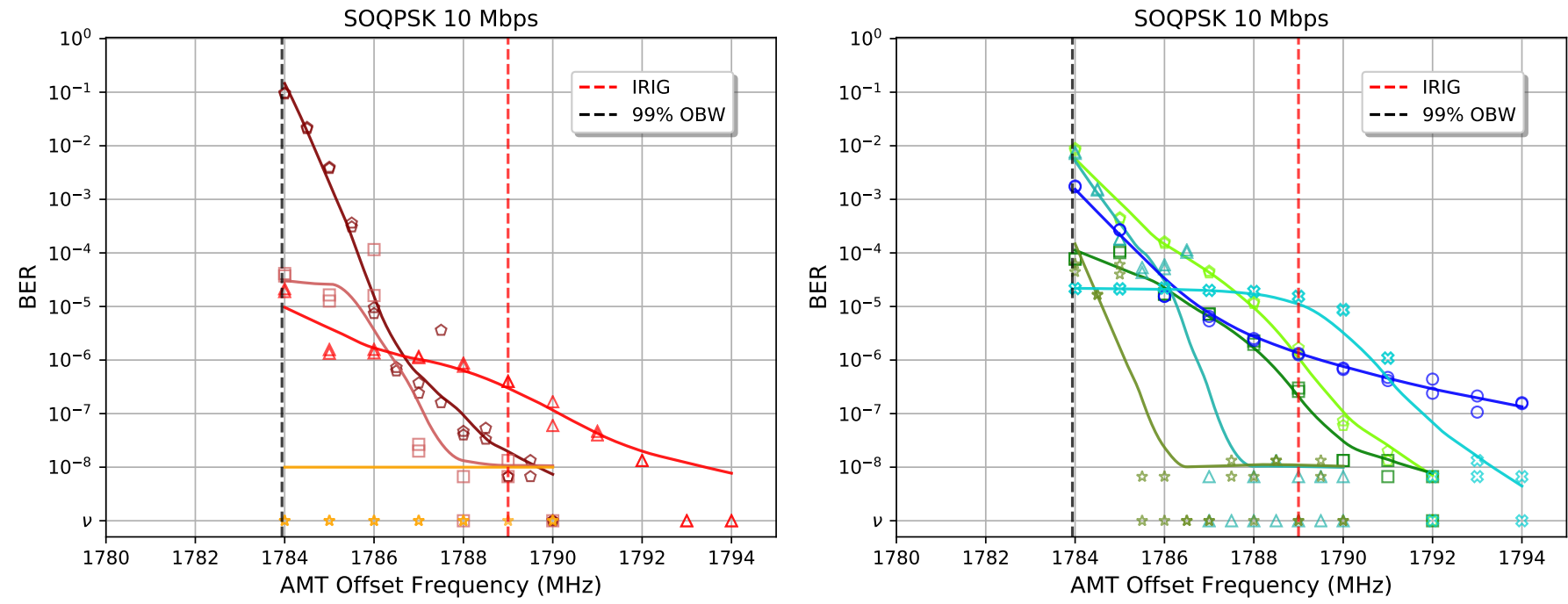

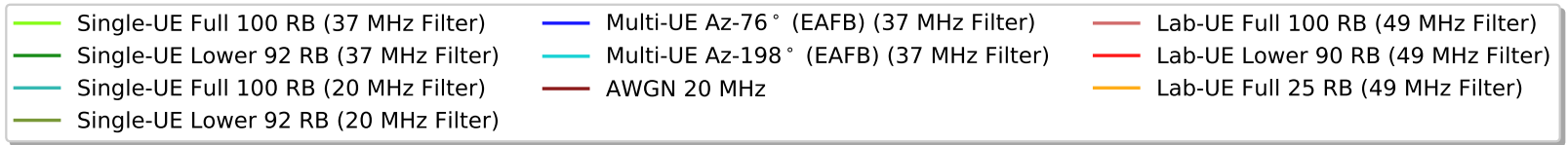

Figure 5.13: BER vs AMT Offset Frequency for all ABE types: SOQPSK $10 \mathrm{Mbps}$, (left) response due to laboratory ABE types, (right) due to in-situ ABE types.
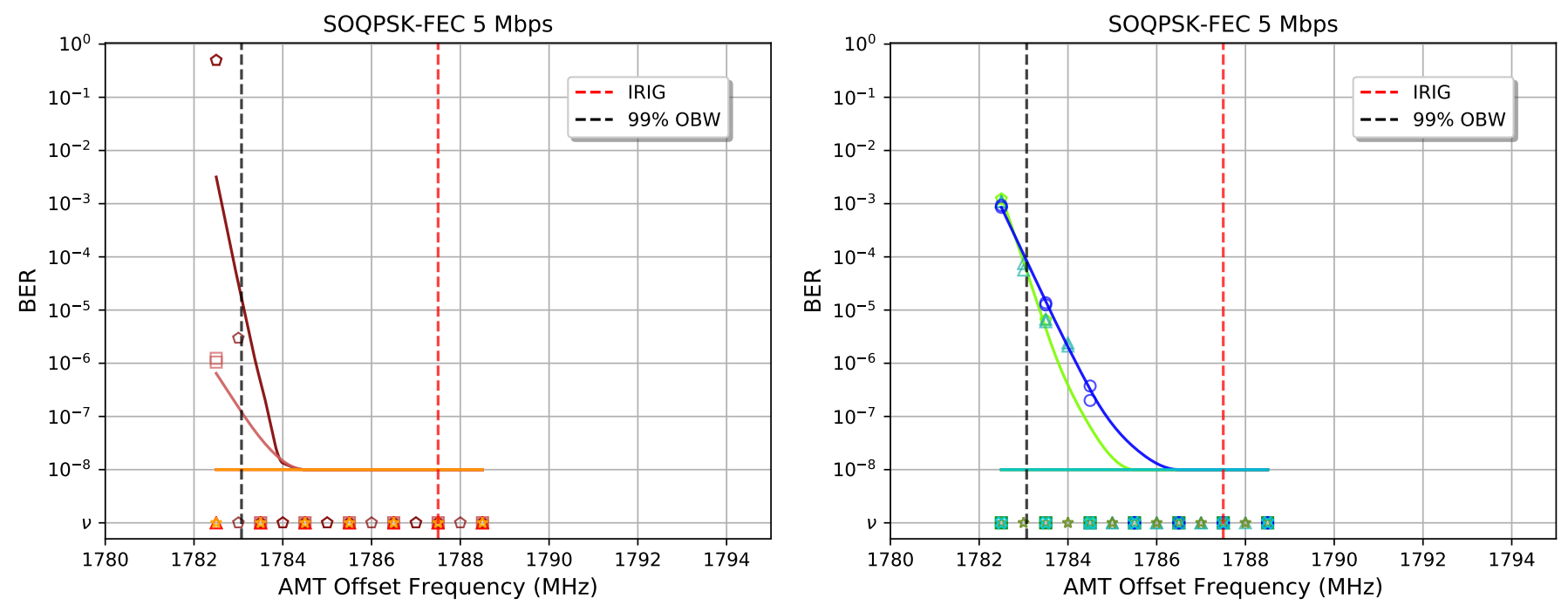

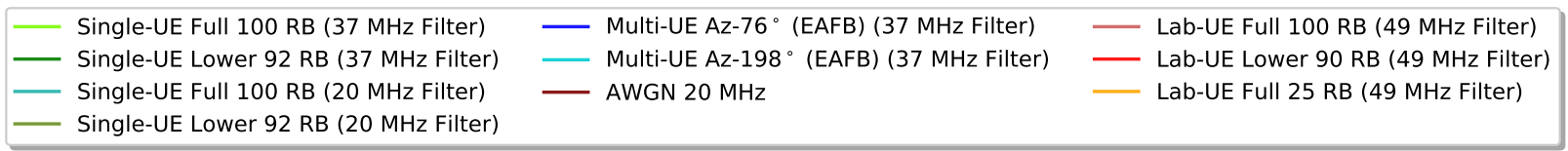

Figure 5.14: BER vs AMT Offset Frequency for all ABE types: SOQPSK-FEC 5 Mbps, (left) response due to laboratory $\mathrm{ABE}$ types, (right) due to in-situ ABE types. 

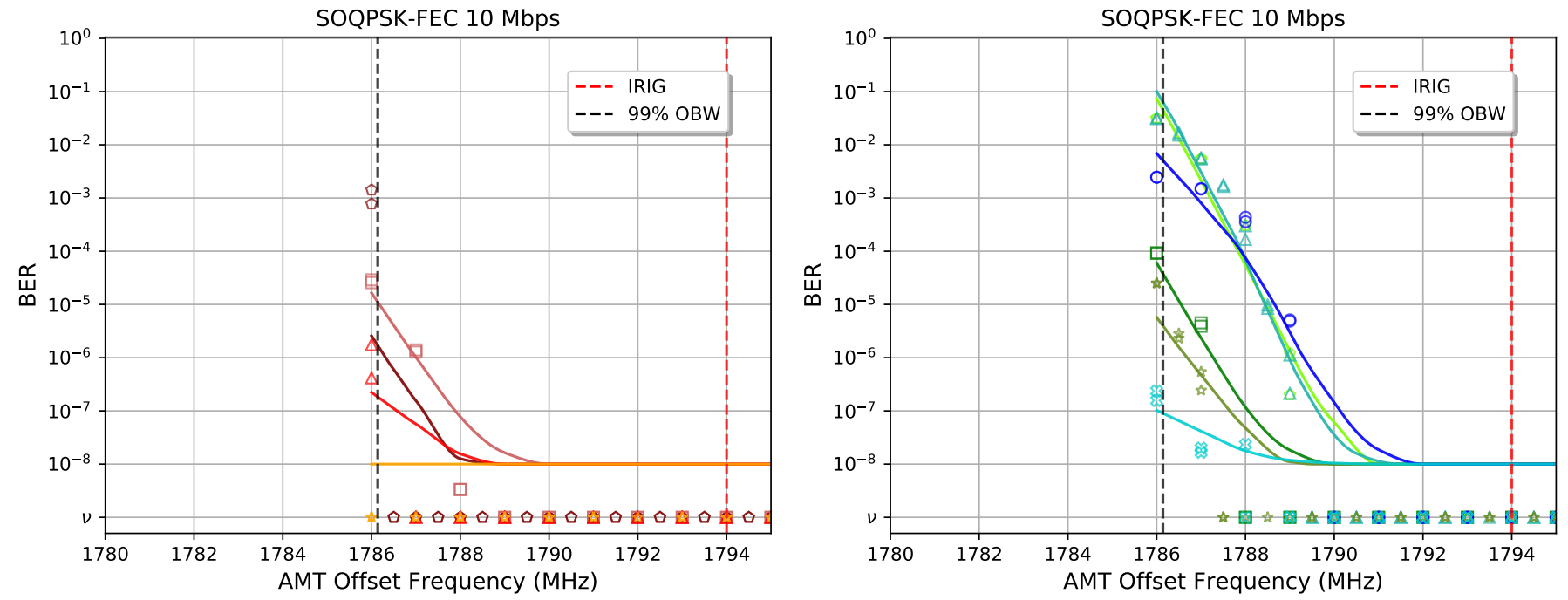

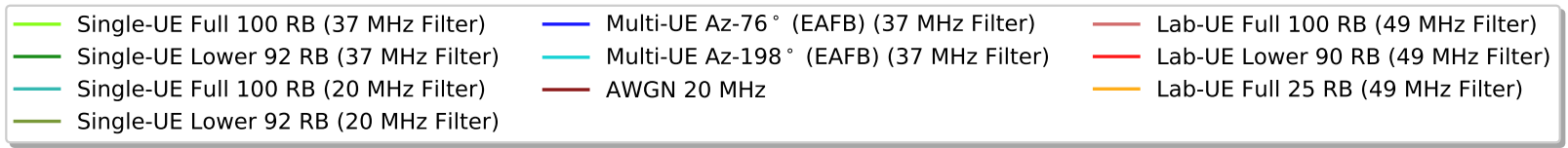

Figure 5.15: BER vs AMT Offset Frequency for all ABE types: SOQPSK-FEC 10 Mbps, (left) response due to laboratory ABE types, (right) due to in-situ ABE types.
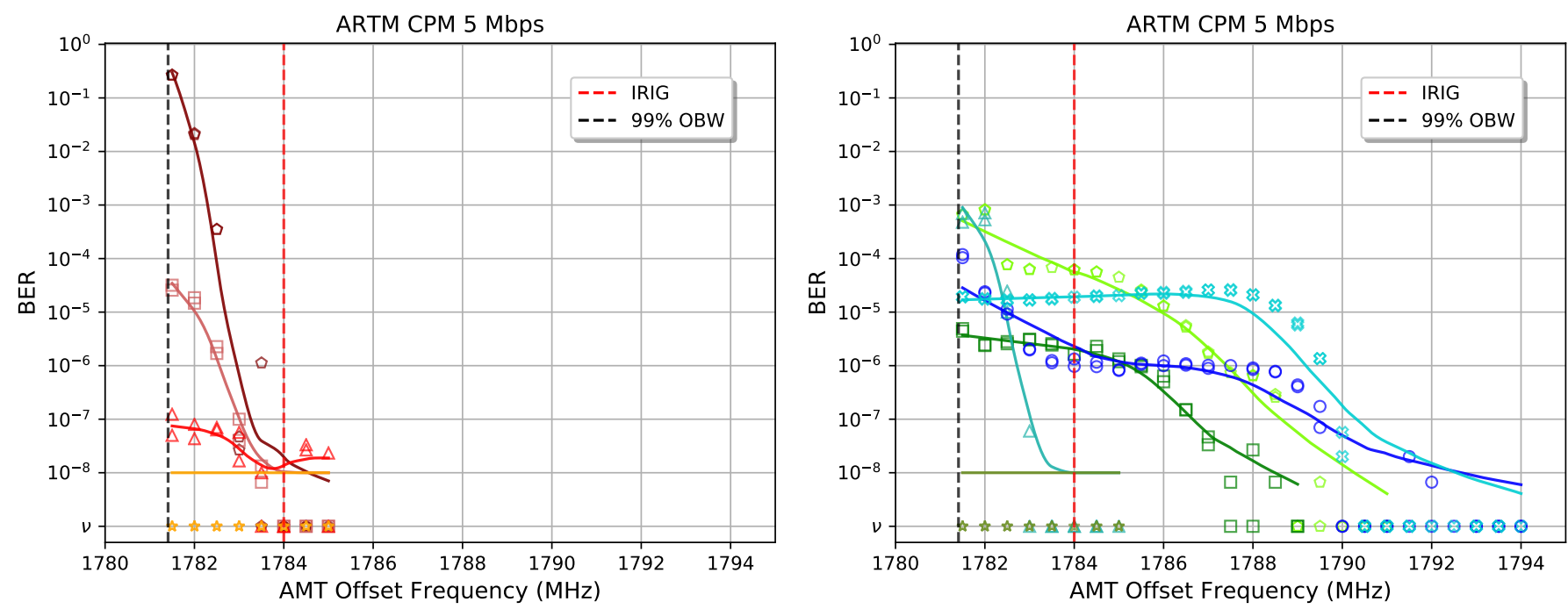

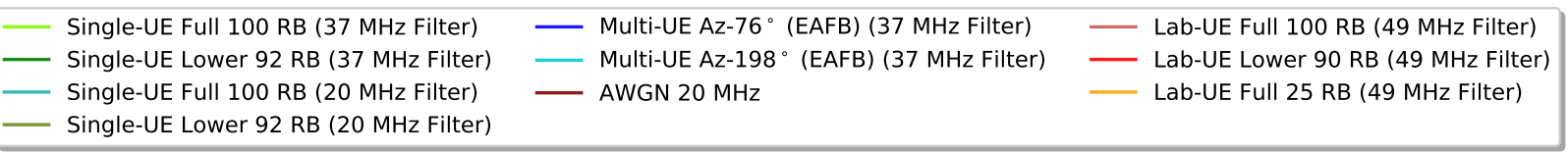

Figure 5.16: BER vs AMT Offset Frequency for all ABE types: ARTM CPM 5 Mbps, (left) response due to laboratory $\mathrm{ABE}$ types, (right) due to in-situ $\mathrm{ABE}$ types. 


\subsection{Discussion of Notable and Anomalous Results}

The measurement data reveal several results warranting additional discussion. Notable and anomalous results include unexpected receiver-under-test behavior, impacts due to the presence of spurious OOBEs, and differences due to ABE waveform processing.

\subsubsection{Unexpected Receiver Response}

Figure 5.1 shows an anomalous response near $-98 \mathrm{dBm}$ AMT RX power. This phenomena can be observed in greater detail by examining Figures B.1,B.2,B.7, and B.8. The unexpected response is exhibited by AMT data rates of 1 Mbps and is observed for PCM/FM and the SOQPSK AMT signals. The impact of this effect is most pronounced in descending order for $\mathrm{ABE}$ waveforms and correlates well with the ABE RMS power tabulated in Table 5.1:

- Single-UE Full 100 RB (EAFB, 20 MHz Filter),

- Single-UE Lower 92 RB (EAFB, 20 MHz Filter),

- AWGN $18 \mathrm{MHz}$,

- Multi-UE Az-76 $100 \mathrm{RB}$ (EAFB, $20 \mathrm{MHz}$ Filter),

- AWGN 16.5 MHz,

- Multi-UE Az-140 $100 \mathrm{RB}$ (LARC, $20 \mathrm{MHz}$ Filter), and

- Multi-UE Az-165 $100 \mathrm{RB}$ (LARC, $20 \mathrm{MHz}$ Filter).

Figure 5.17 shows results from additional test runs on the Bedford testbed for the AWGN $18 \mathrm{MHz}$ ABE waveform, with with a smaller step-size for the AMT RX power sweep. These additional tests include results for both AMT RX input channels. The effect is present in both channels but is shifted by $2 \mathrm{~dB}$ in Channel 2 .

Note that the NASCTN Boulder testbed does not demonstrate the behavior as evidenced by Figure 5.18. The measurements occur over several different rounds, ruling out non-systematic issues within testbed automation. The AMT RX implementation is possibly the cause of the anomalous behavior.

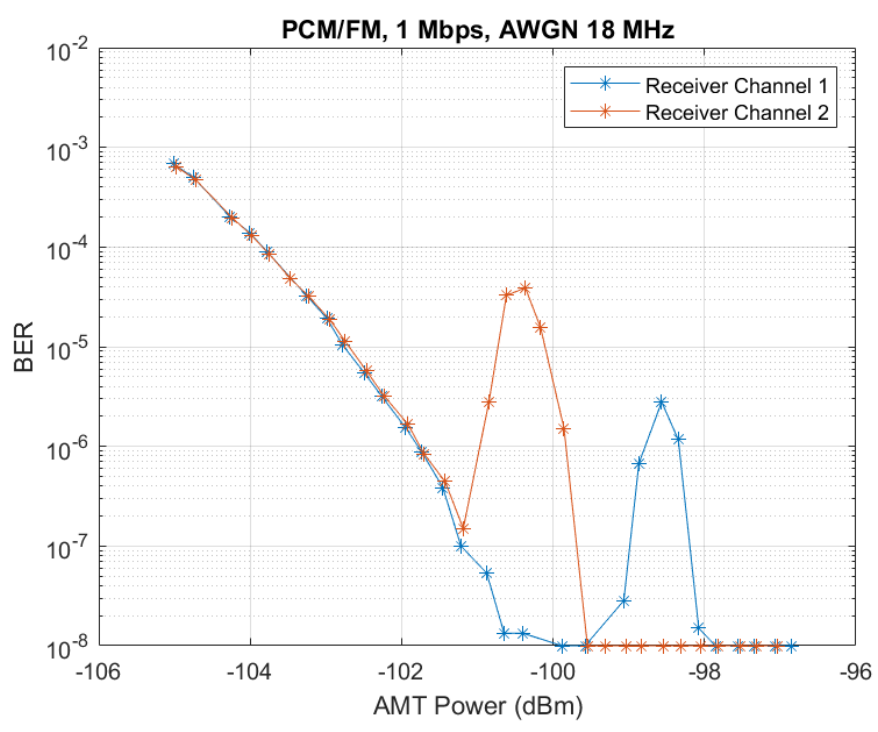

Figure 5.17: In depth investigation of the anomalous results observed at the Bedford testbed for $1 \mathrm{Mbps}$ data rate PCM/FM waveform. The median of two consecutive test runs on AMT RX channel 1 and on AMT RX channel 2 shows a clear effect on the receiver performance. 

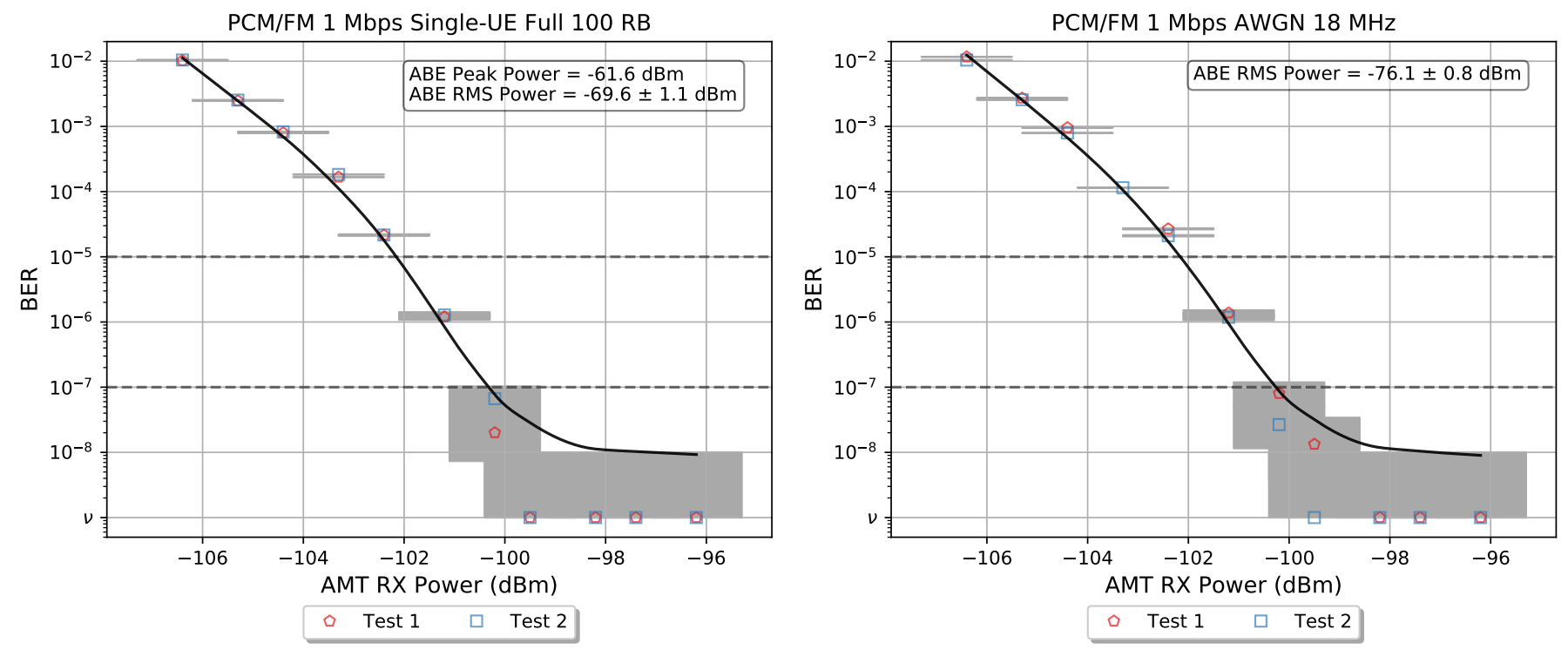

Figure 5.18: Anomalous Results Investigation: Boulder Testbed. BER vs AMT RX Power for ABE types: Single-UE Full $100 \mathrm{RB}$ (left) and AWGN $18 \mathrm{MHz}$ (right). Note that the Boulder testbed does not exhibit the anomalous behavior near $-98 \mathrm{dBm}$.

\subsubsection{Spurious OOBE Activity}

Another observed anomaly is the slow convergence to a $B E R=10^{-8}$ in several of the frequency offset results for the Multi-UE Az-198 (EAFB, $37 \mathrm{MHz}$ Filter). For example, see Figures 5.11 and 5.14, where the curves for the the Multi-UE Az-198 ${ }^{\circ}$ (EAFB, $37 \mathrm{MHz}$ Filter) case do not converge as rapidly to a BER $=10^{-8}$ as compared to the MultiUE Az-76 ${ }^{\circ}$ (EAFB, $37 \mathrm{MHz}$ Filter) cases. This is due to additional OOBEs that do not correlate with the fundamental LTE emissions.

Figure 5.19 and Figure 5.20 show the persistence spectra and spectrograms with the LTE resource grid overlaid for these $\mathrm{ABE}$ waveforms. A close examination of the persistence spectra reveals the presence of spurious OOBEs in the AMT band for the Multi-UE Az- $198^{\circ}$ waveform, whereas spurious OOBEs for the Multi-UE Az- $76^{\circ}$ waveform are barely discernable. However, in both figures, the time segments do not show correlation between the OOBE in the AMT band and the LTE transmissions as delineated by the LTE grid overlays. Since these are in situ waveforms, these spurious OOBEs could be due to an unidentified, non-LTE transmitter. (These waveforms are translated from the in situ baseband captures to a center frequency of $1770 \mathrm{MHz}$ for playback in the testbed.)

\subsubsection{ABE Waveform Processing}

Finally, besides the choice of which $\mathrm{ABE}$ waveforms to test against, processing decisions are necessary to prepare the $\mathrm{ABE}$ waveforms for playback in the testbed. The differences between a $20 \mathrm{MHz}$ and a $37 \mathrm{MHz}$ filtering of the in-situ captures are significant in some of the results due to the OOBE associated with the captures. 


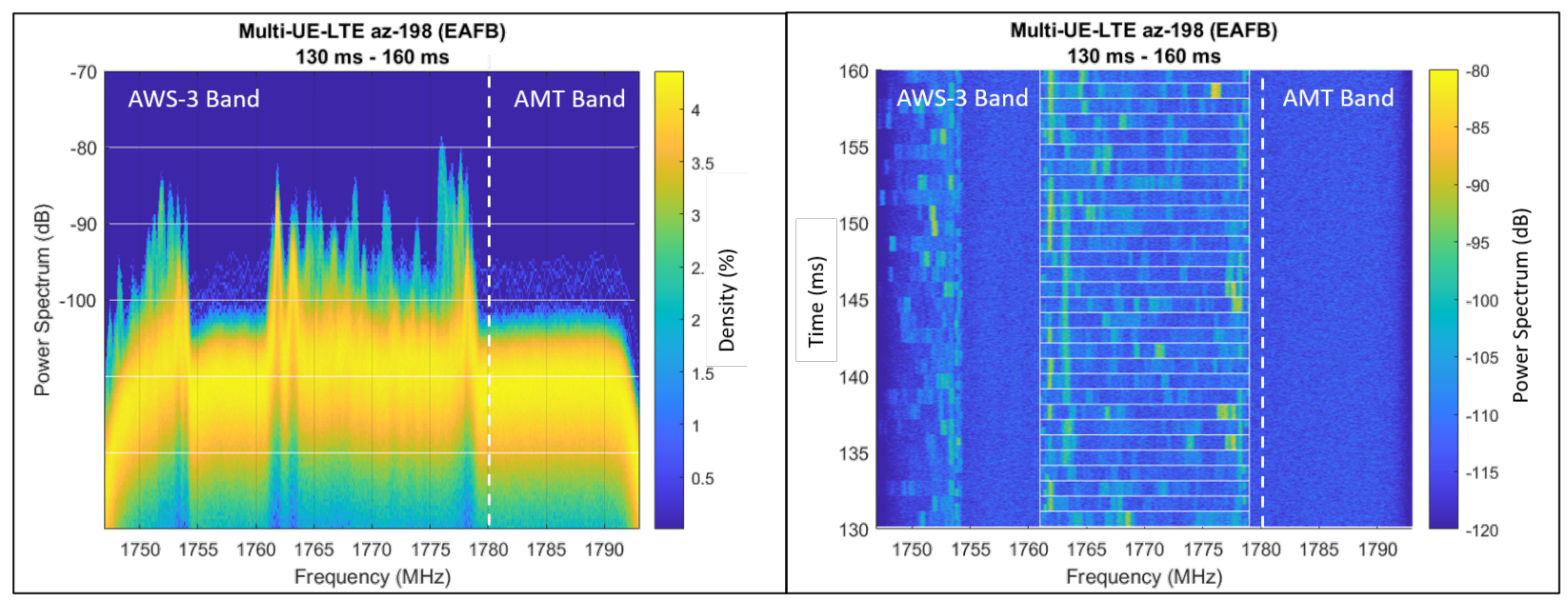

Figure 5.19: Persistence spectrum and LTE timing grid overlay for the in-situ Multi-UE Az- $198^{\circ}$ (EAFB, $37 \mathrm{MHz}$ Filter). (Different color scales are used for the persistent spectrum on the left and the time segment on the right to improve visualization of the OOBEs)
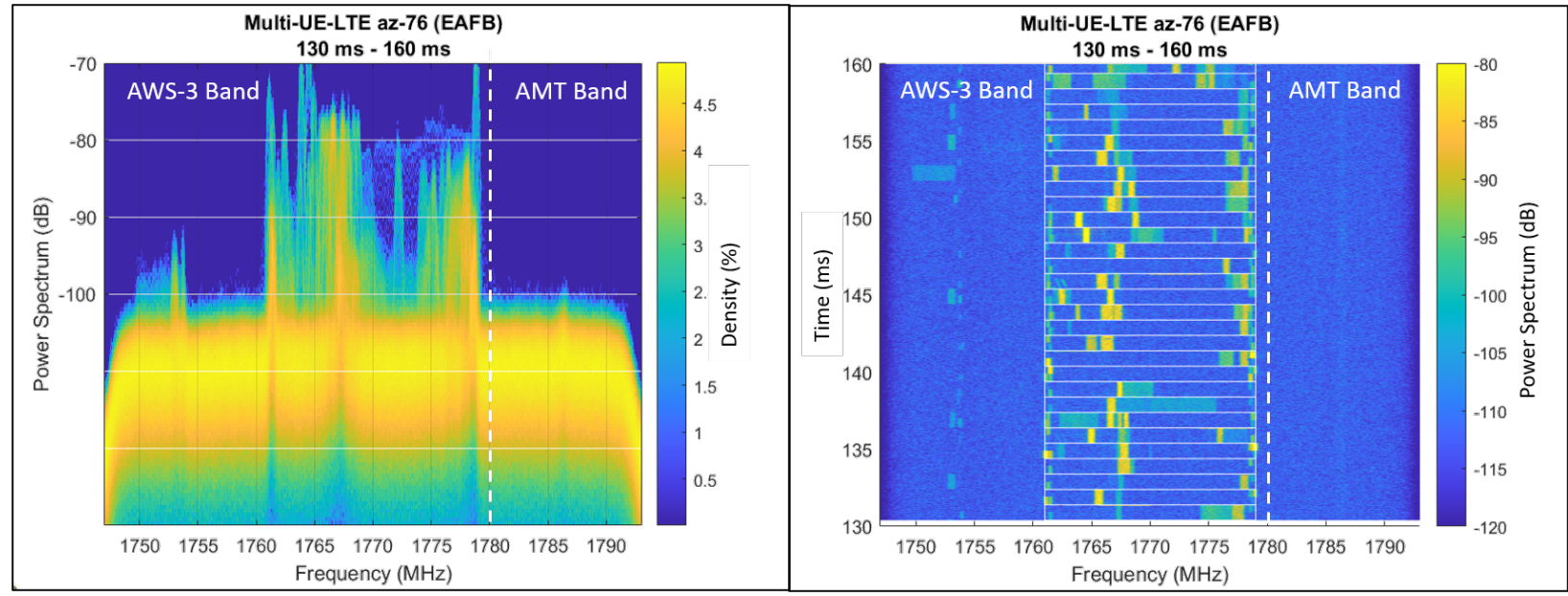

Figure 5.20: Persistence spectrum and LTE timing grid overlay for the in-situ Multi-UE Az-76 ${ }^{\circ}$ EAFB, $37 \mathrm{MHz}$ Filter). (Different color scales are used for the persistent spectrum on the left and the time segment on the right to improve visualization of the OOBEs) 


\section{Chapter 6}

\section{Secondary Data Analysis Results}

This chapter presents additional results beyond the primary results in Chapter 5, including additional bit rates and side experiment investigations on the impacts due to equalization and space-time coding.

Finally, Section 6.4 compares results from the Bedford and Boulder testbeds for several select cases. Most of the results between the testbeds are consistent. However, some example cases of inconsistency between the testbeds are shown as well.

\subsection{Main Experiment: Additional Configurations}

This section presents results for the configurations collected in Bedford that were not presented in the prior chapter. Namely, results for the non-highlighted configurations in Table 2.4 are given, including additional bit rates of 10 Mbps for PCM/FM, 1 and 20 Mbps for SOQPSK, 1 and 20 Mbps for SOQPSK-FEC, and 10 and 20 Mbps for ARTM CPM.

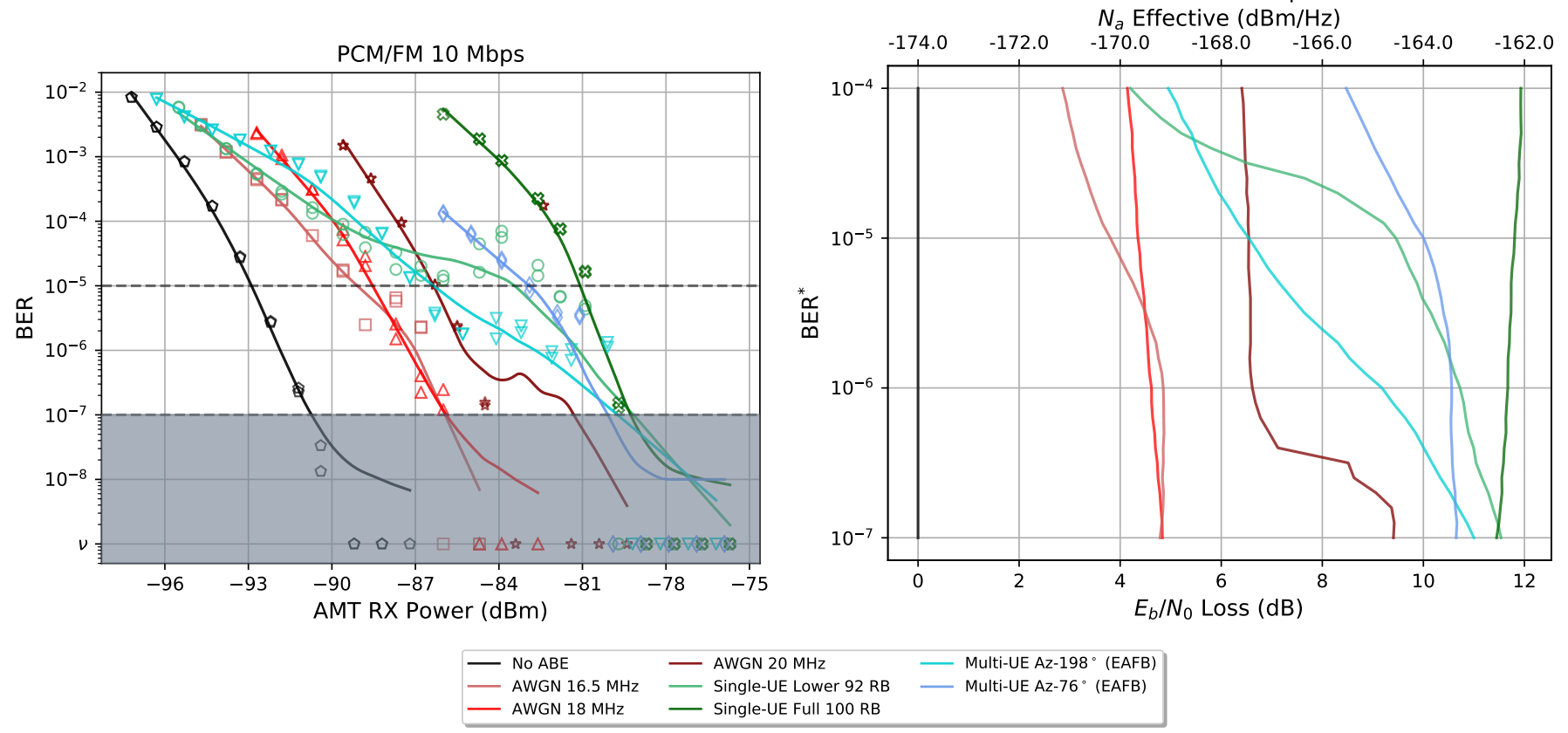

Figure 6.1: BER vs AMT RX power and BER* vs $N_{a}$ Effective for selected ABE types: PCM/FM 10 Mbps. The region $\mathrm{BER}<10^{-7}$ is shaded in the left plot to indicate high relative uncertainty. 


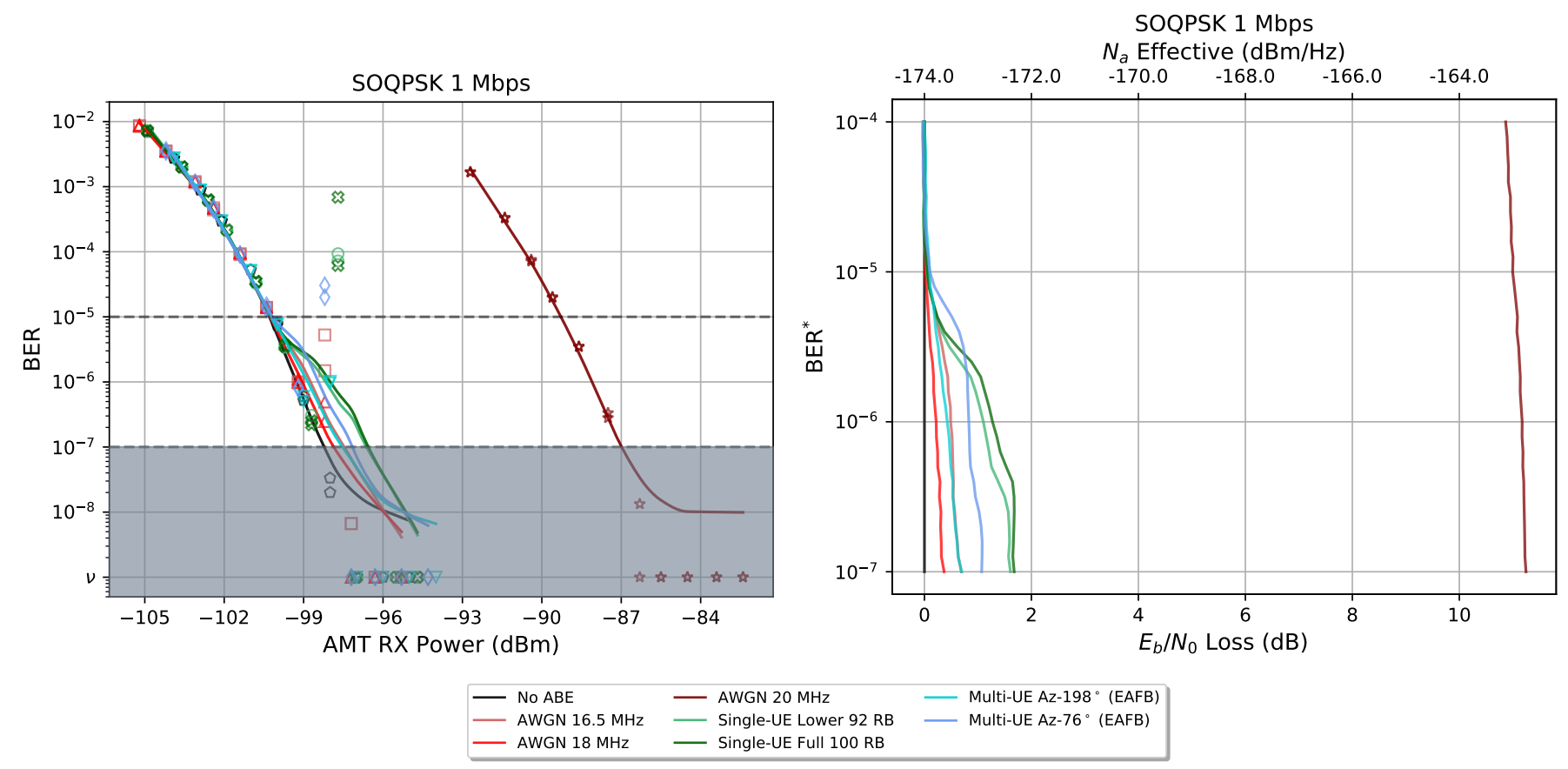

Figure 6.2: BER vs AMT RX power and BER* vs $N_{a}$ Effective for selected ABE types: SOQPSK 1 Mbps. The region $\mathrm{BER}<10^{-7}$ is shaded in the left plot to indicate high relative uncertainty.

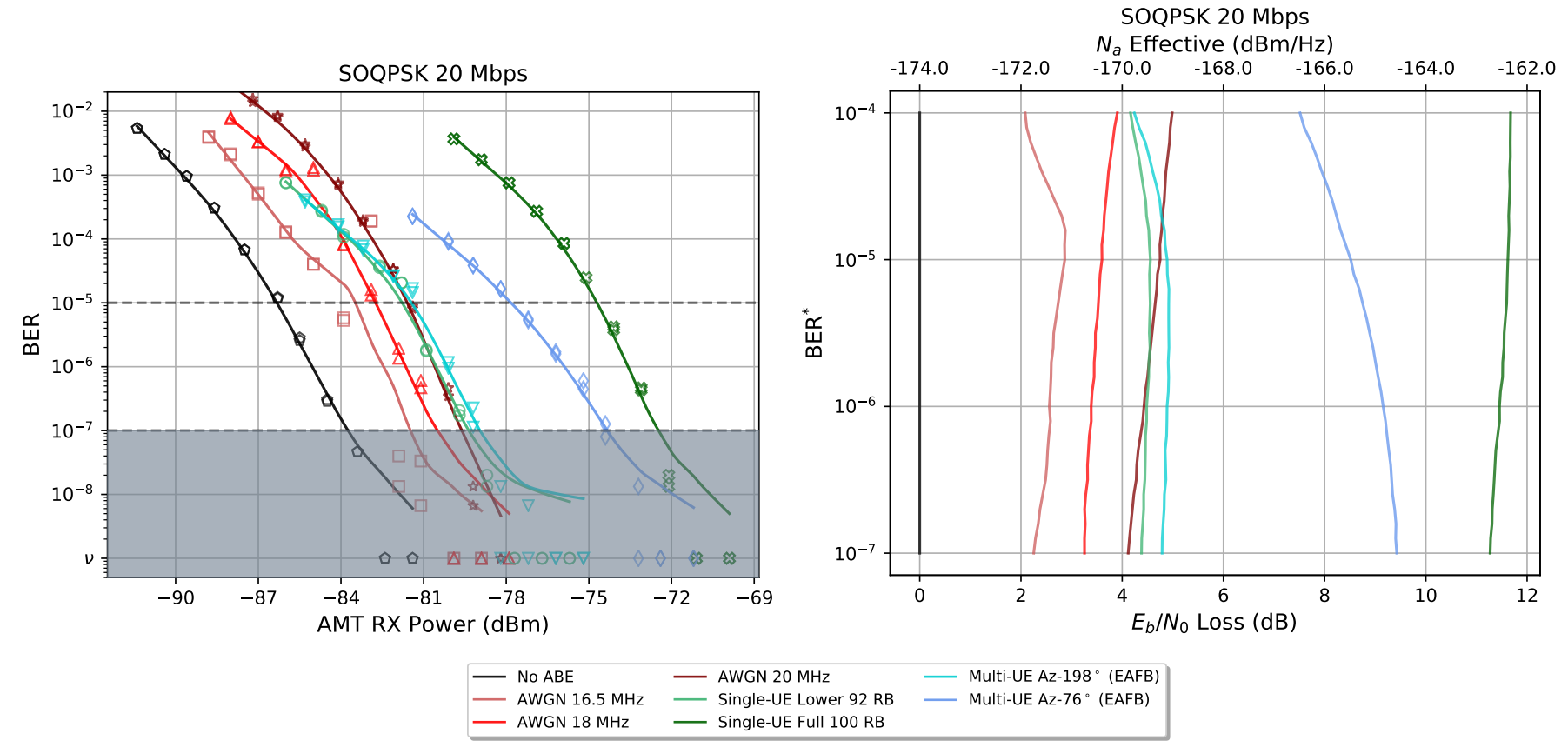

Figure 6.3: BER vs AMT RX power and BER* vs $N_{a}$ Effective for selected ABE types: SOQPSK 20 Mbps. The region $\mathrm{BER}<10^{-7}$ is shaded in the left plot to indicate high relative uncertainty. 


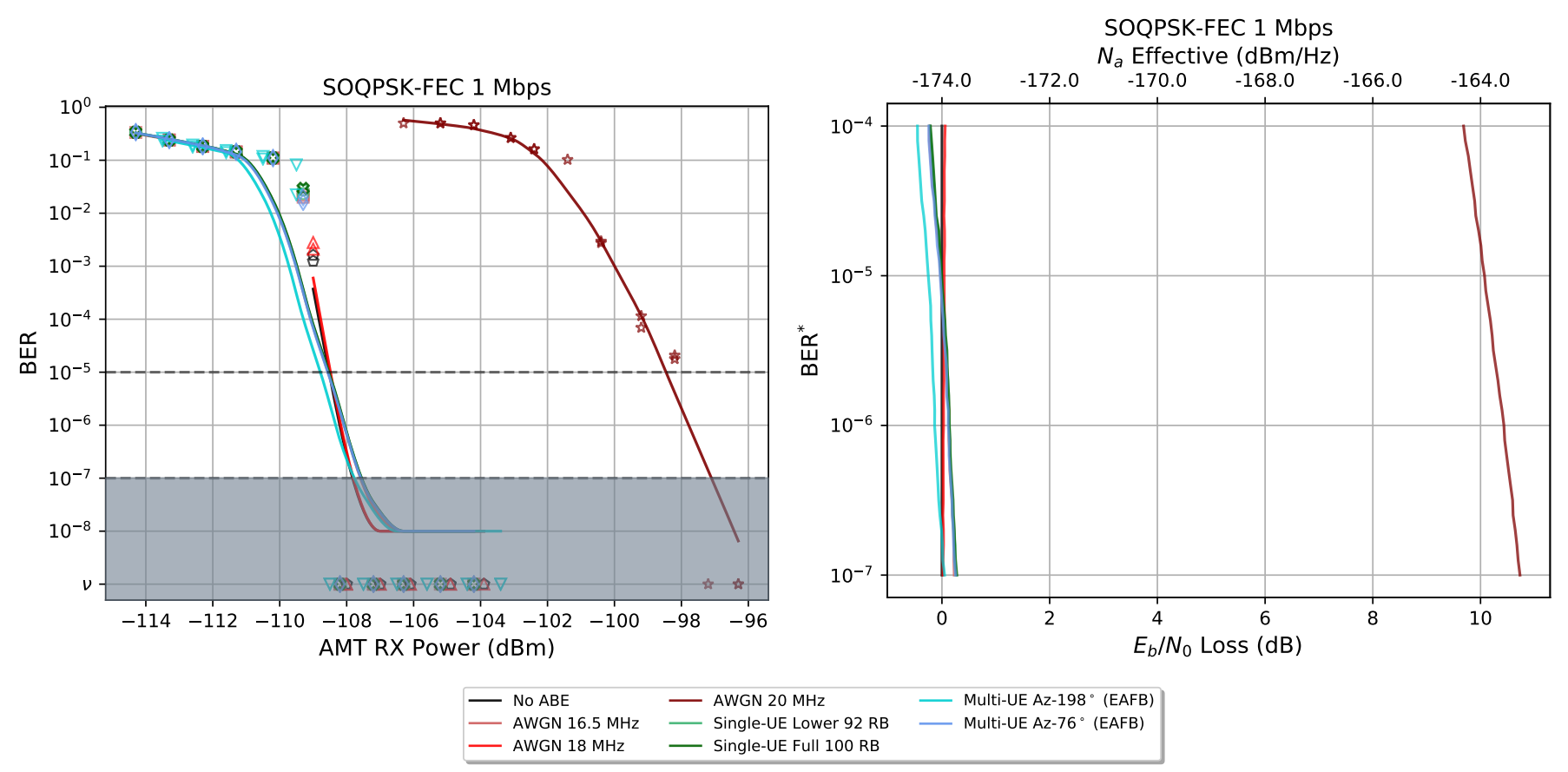

Figure 6.4: BER vs AMT RX power and BER* vs $N_{a}$ Effective for selected ABE types: SOQPSK-FEC 1 Mbps. The region $\mathrm{BER}<10^{-7}$ is shaded in the left plot to indicate high relative uncertainty.
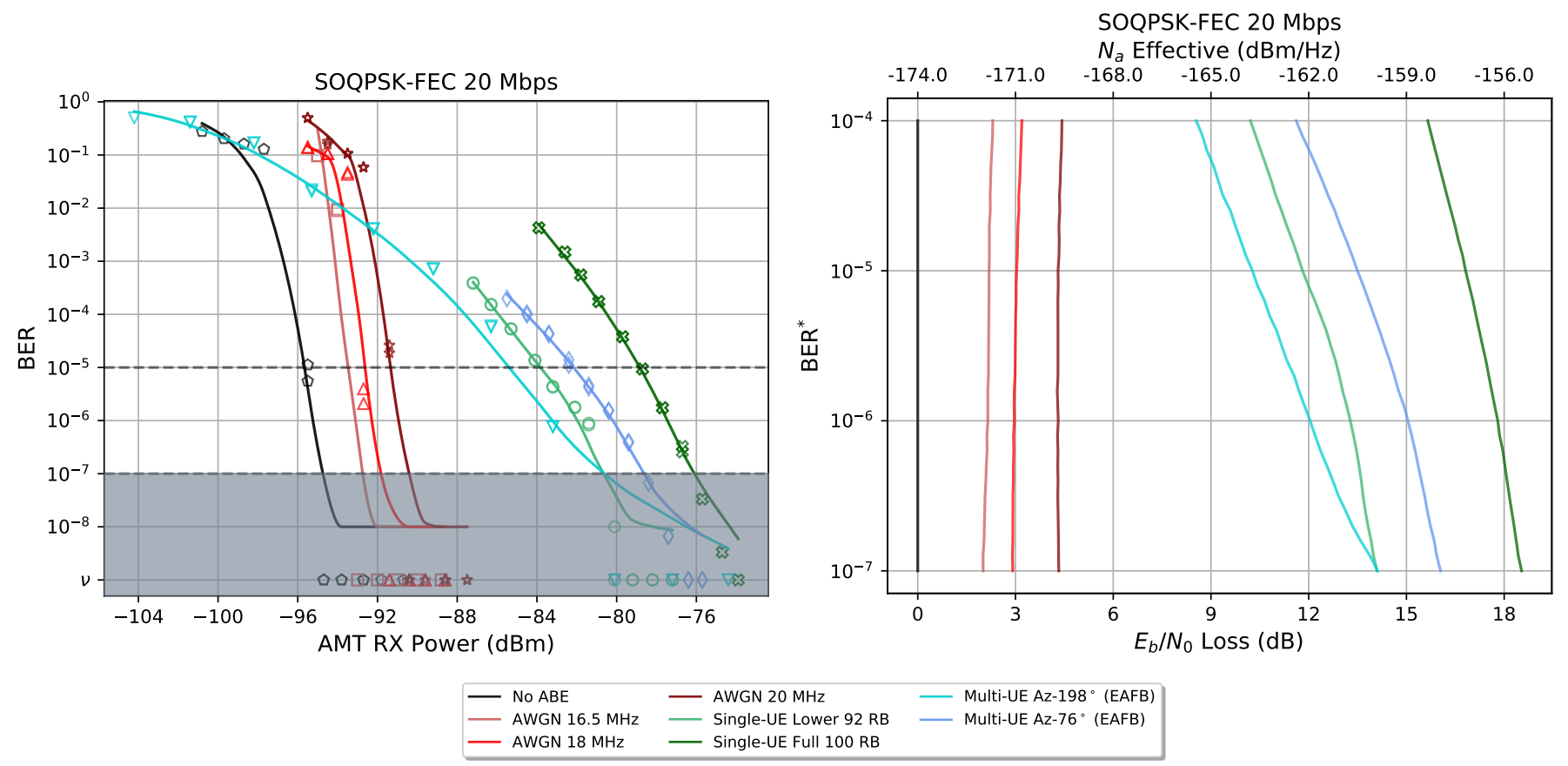

Figure 6.5: BER vs AMT RX power and BER* vs $N_{a}$ Effective for selected ABE types: SOQPSK-FEC 20 Mbps. The region $\mathrm{BER}<10^{-7}$ is shaded in the left plot to indicate high relative uncertainty. 

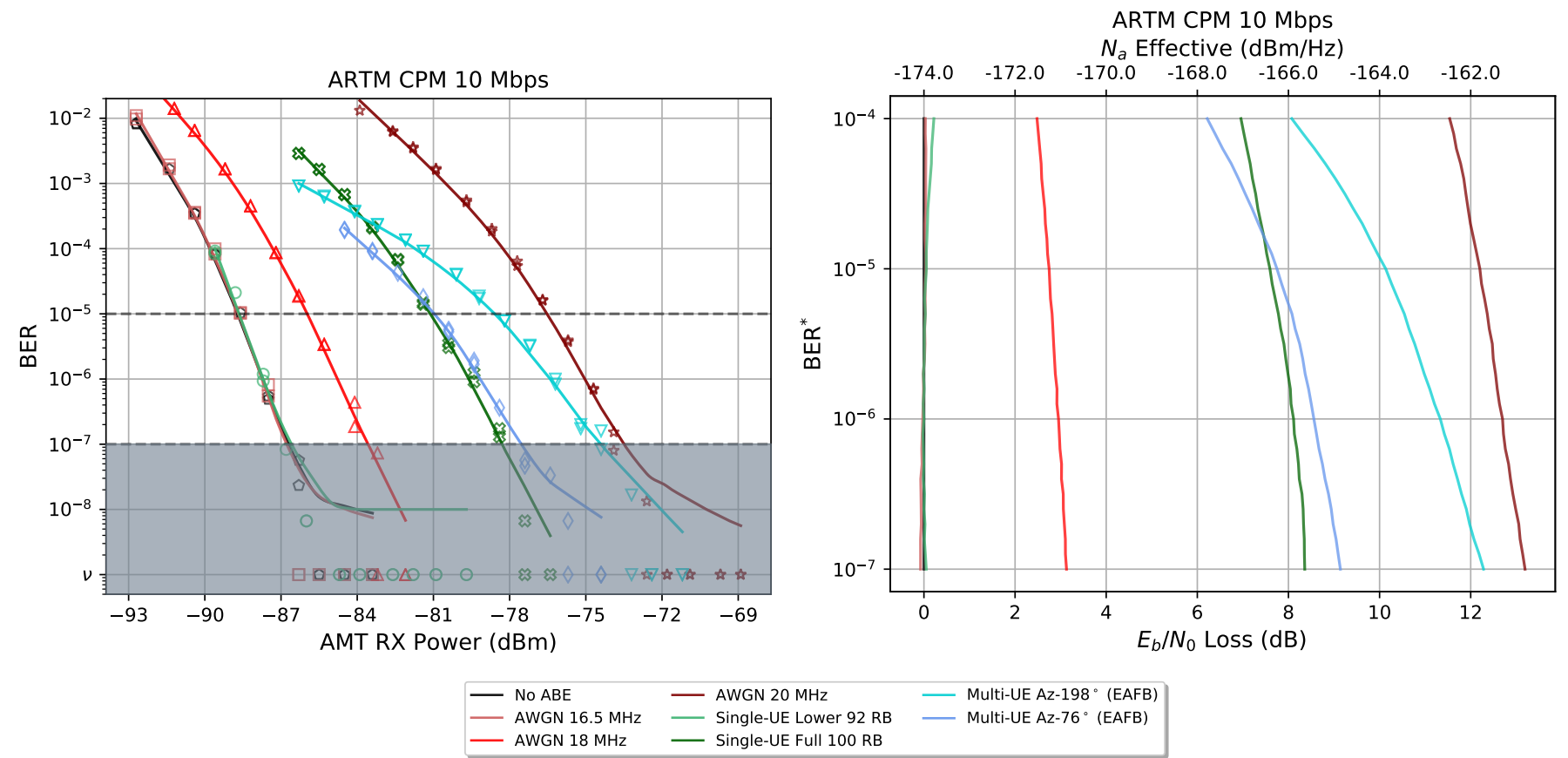

Figure 6.6: BER vs AMT RX power and BER* vs $N_{a}$ Effective for selected ABE types: ARTM CPM 10 Mbps. The region $\mathrm{BER}<10^{-7}$ is shaded in the left plot to indicate high relative uncertainty.
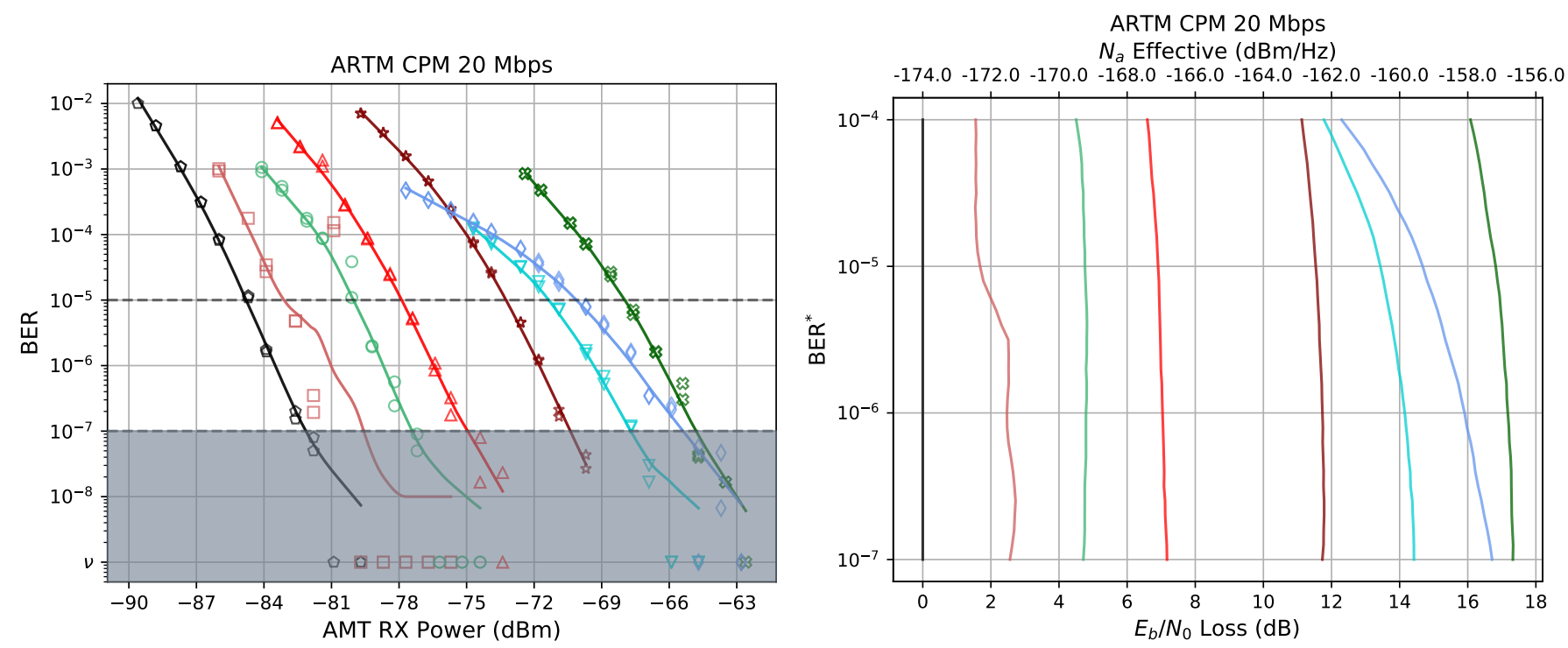

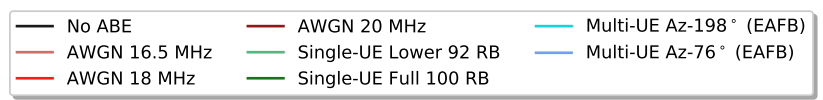

Figure 6.7: BER vs AMT RX power and BER* vs $N_{a}$ Effective for selected ABE types: ARTM CPM 20 Mbps. The region $\mathrm{BER}<10^{-7}$ is shaded in the left plot to indicate high relative uncertainty. 


\subsection{Side-Experiment A: Equalizer Test}

This section presents results for Side Experiment A, described in Section 2.5.1, which investigates the impacts of turning on equalization in the AMT receiver. Only the $5 \mathrm{Mbps}$ case is covered. The differences between between when the equalizer is on or off is most noticeable in the Mutli-UE and Single-UE Full 100 RB ABE cases.
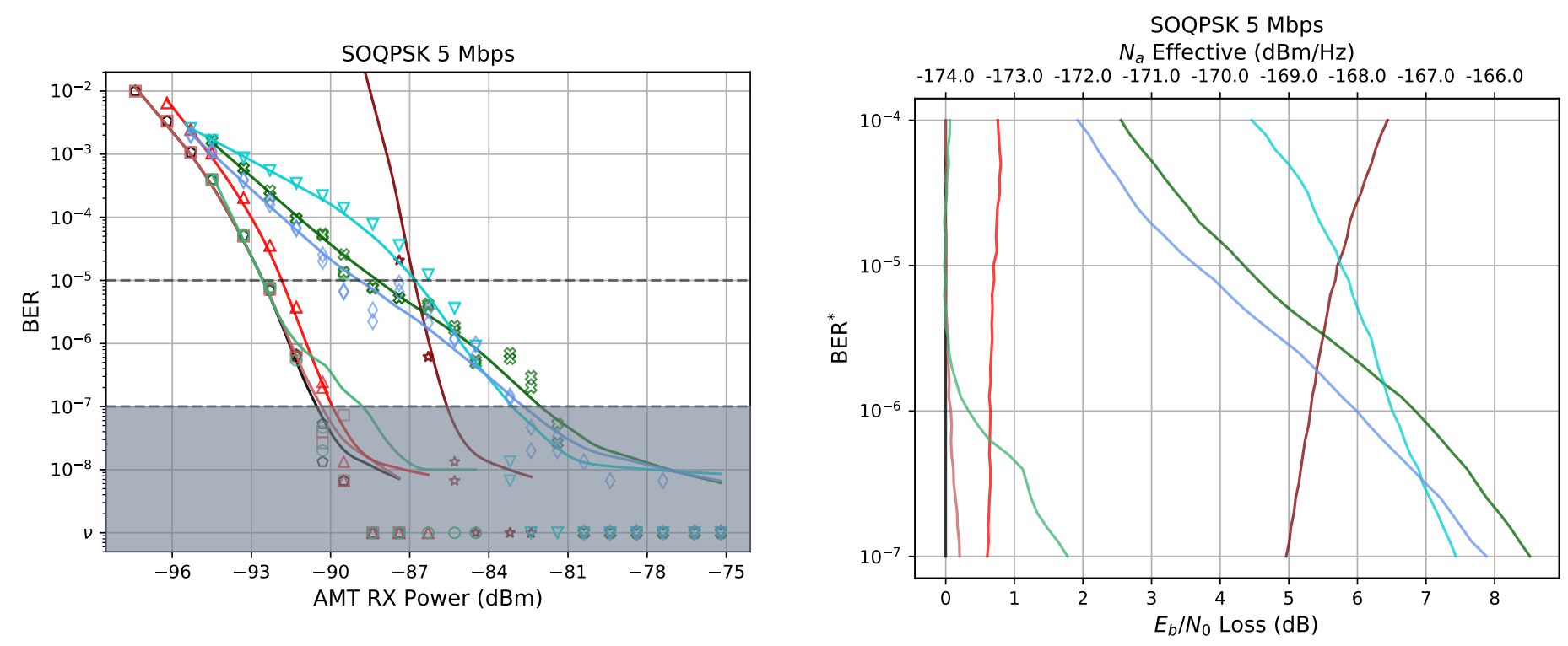

\begin{tabular}{lll}
- No ABE & - AWGN 20 MHz & - Multi-UE Az-198 ${ }^{\circ}$ (EAFB) \\
AWGN 16.5 MHz & Single-UE Lower 92 RB & Multi-UE Az-76 ${ }^{\circ}$ (EAFB) \\
\hline AWGN 18 MHz & Single-UE Full 100 RB & \\
\hline
\end{tabular}

Figure 6.8: Equalizer On. BER vs AMT RX power for selected ABE types: SOQPSK 5 Mbps. The region BER < $10^{-7}$ is shaded in the left plots to indicate high relative uncertainty. 

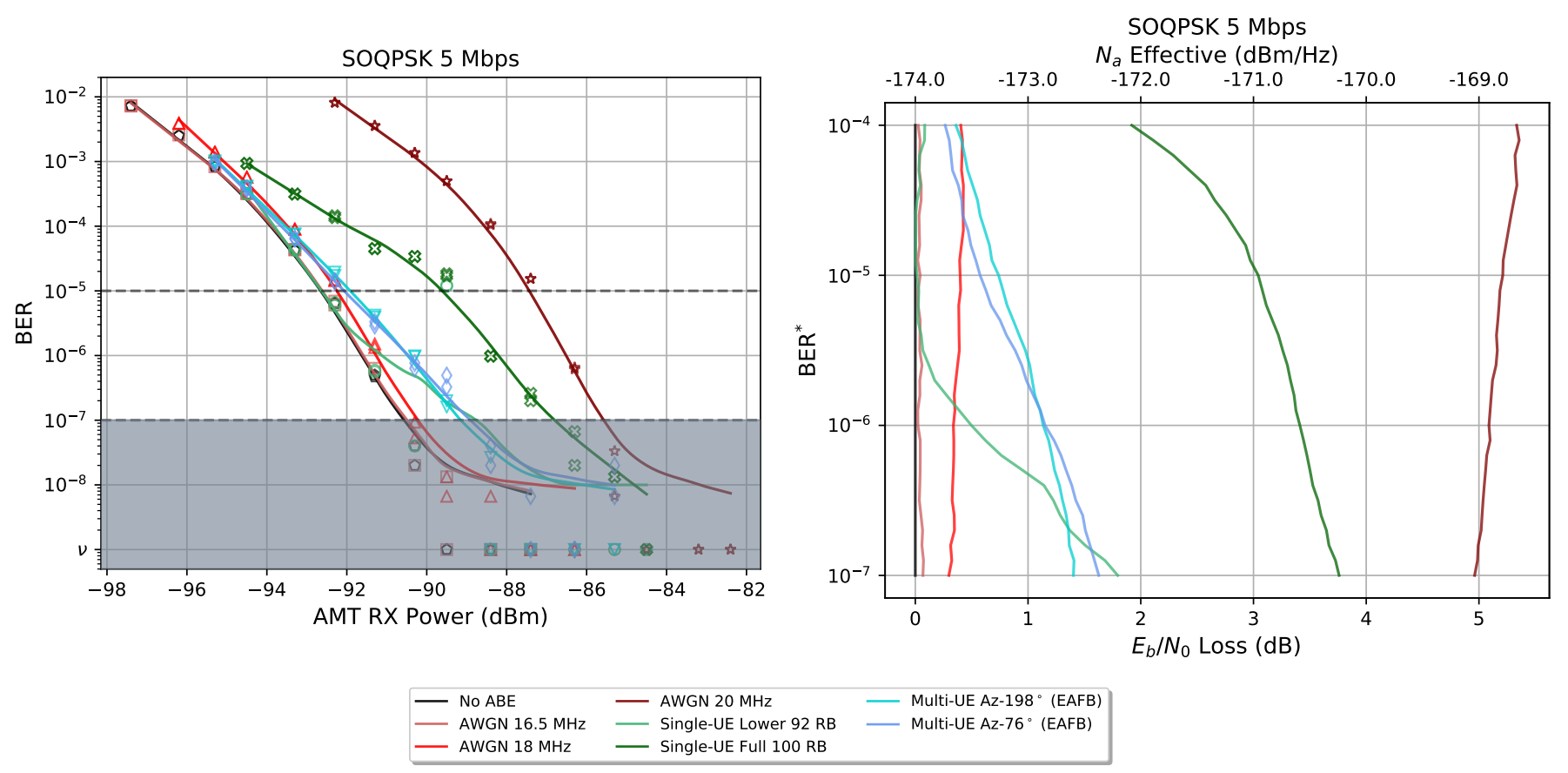

Figure 6.9: Equalizer Off. BER vs AMT RX power for selected ABE types: SOQPSK 5 Mbps. The region BER < $10^{-7}$ is shaded in the left plots to indicate high relative uncertainty. 


\subsection{Side-Experiment B: Space-Time Coding}

The results presented here are from Side Experiment B, described in Section 2.5.2, which investigates the impacts when using STC for the AMT modulation. These results can be compared to the same set of ABE impacts for PCM/FM, SOQPSK, and ARTM CPM at bit rates of 5, 10 and 20 Mbps.

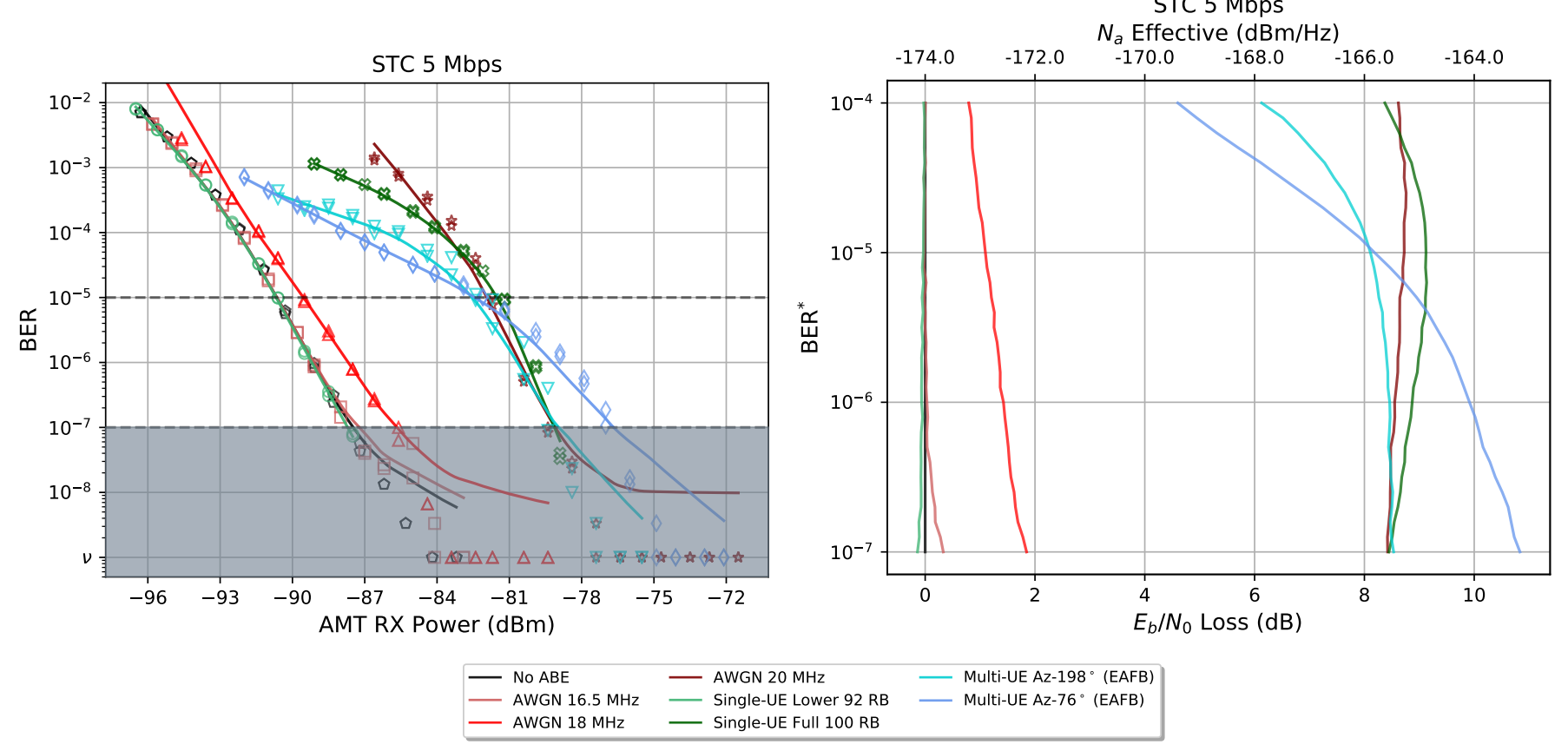

Figure 6.10: BER vs AMT RX power and BER* vs $N_{a}$ Effective for selected ABE types: STC 5 Mbps. The region BER $<10^{-7}$ is shaded in the left plot to indicate high relative uncertainty. 
STC $10 \mathrm{Mbps}$

$N_{a}$ Effective $(\mathrm{dBm} / \mathrm{Hz})$
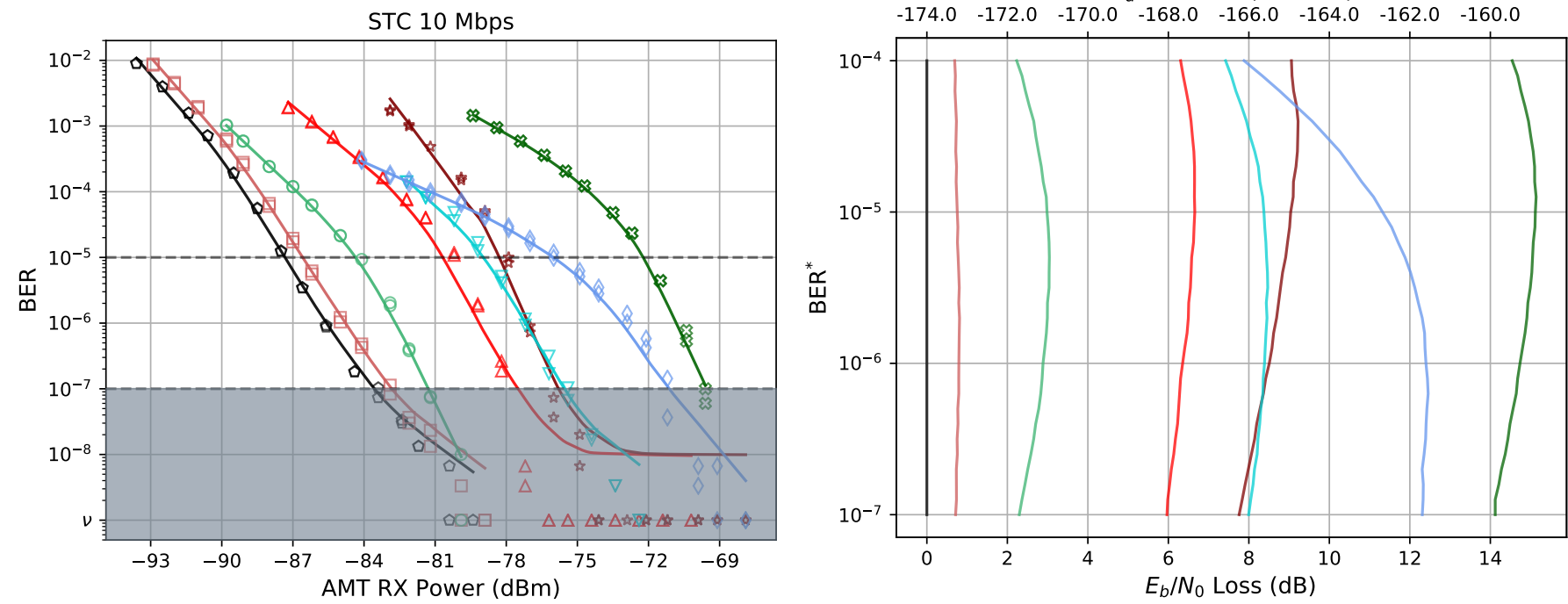

\begin{tabular}{|c|c|c|}
\hline $\begin{array}{l}\text { - No ABE } \\
\text { - AWGN } 16.5 \mathrm{MHz} \\
\text { AWGN } 18 \mathrm{MHz}\end{array}$ & $\begin{array}{l}\text { - AWGN } 20 \mathrm{MHz} \\
\text { - Single-UE Lower } 92 \mathrm{RB} \\
\text { - Single-UE Full } 100 \mathrm{RB}\end{array}$ & $\begin{array}{l}\left.\text { - Multi-UE Az-198 }{ }^{\circ} \text { (EAFB) }\right) \\
\text { - Multi-UE Az-76 }{ }^{\circ} \text { (EAFB) }\end{array}$ \\
\hline
\end{tabular}

Figure 6.11: BER vs AMT RX power and BER* vs $N_{a}$ Effective for selected ABE types: STC 10 Mbps. The region BER $<10^{-7}$ is shaded in the left plot to indicate high relative uncertainty.

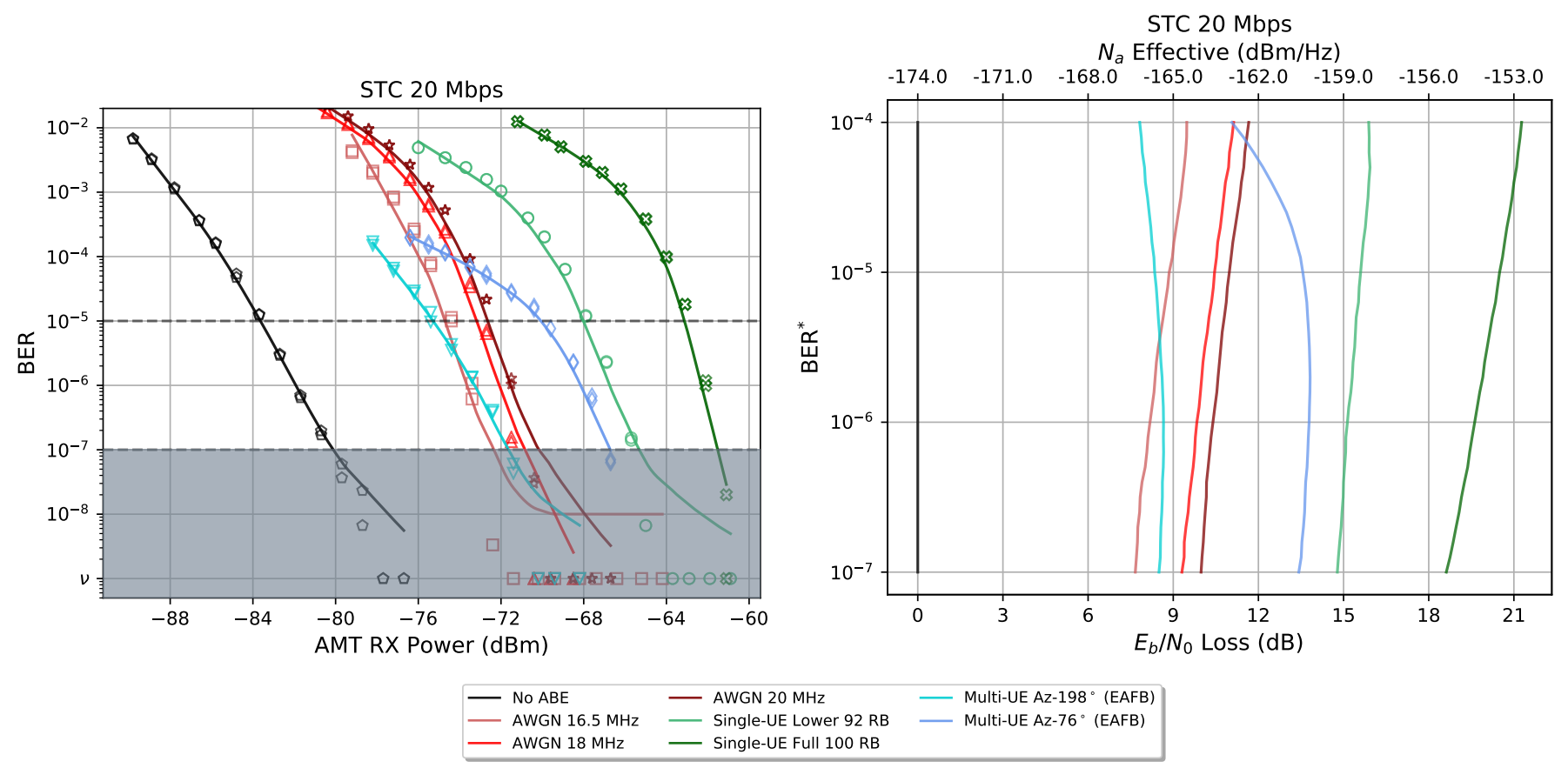

Figure 6.12: BER vs AMT RX power and BER* vs $N_{a}$ Effective for selected ABE types: STC 20 Mbps. The region $\mathrm{BER}<10^{-7}$ is shaded in the left plot to indicate high relative uncertainty. 
STC-FEC $5 \mathrm{Mbps}$
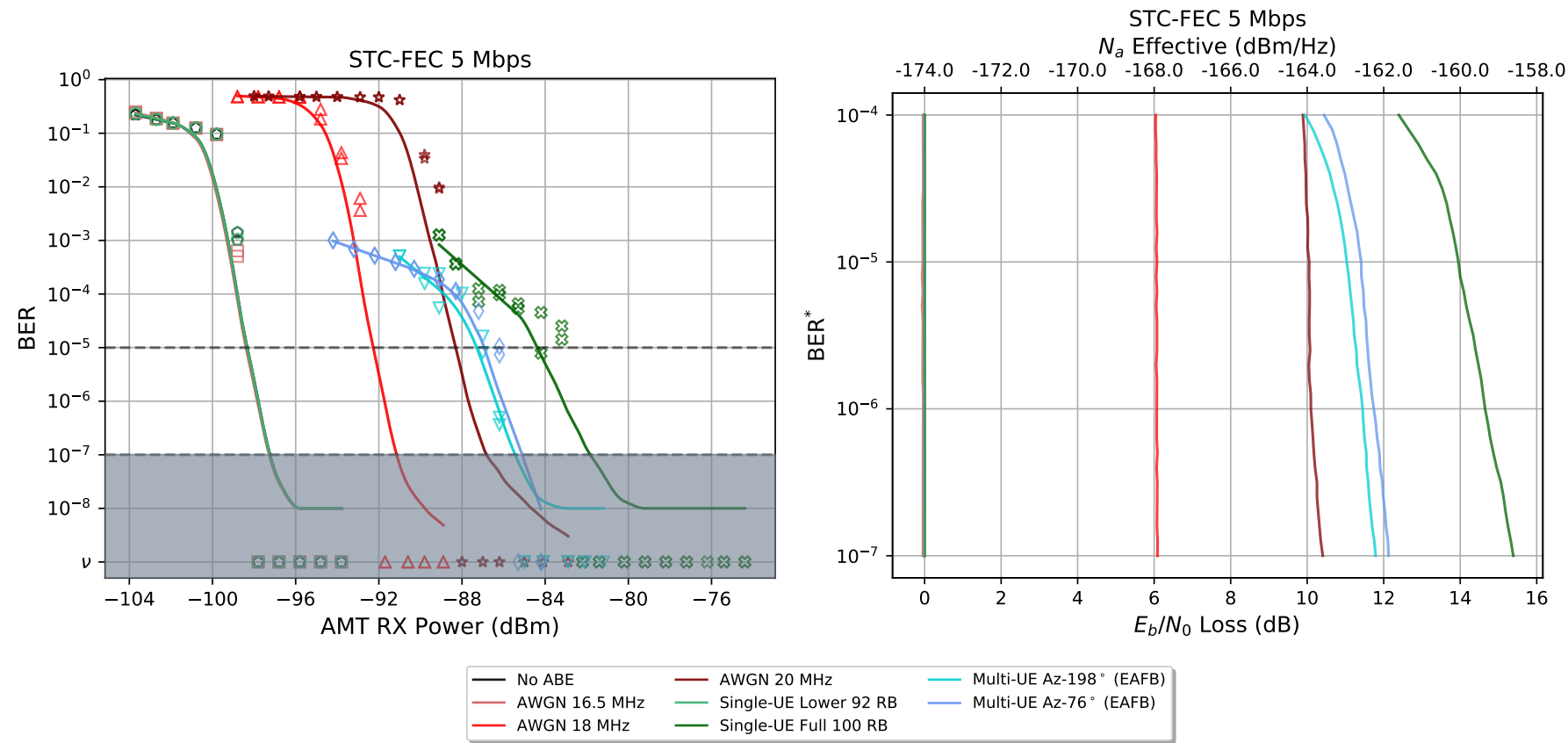

Figure 6.13: BER vs AMT RX power and BER* vs $N_{a}$ Effective for selected ABE types: STC-FEC 5 Mbps. The region $\mathrm{BER}<10^{-7}$ is shaded in the left plot to indicate high relative uncertainty.
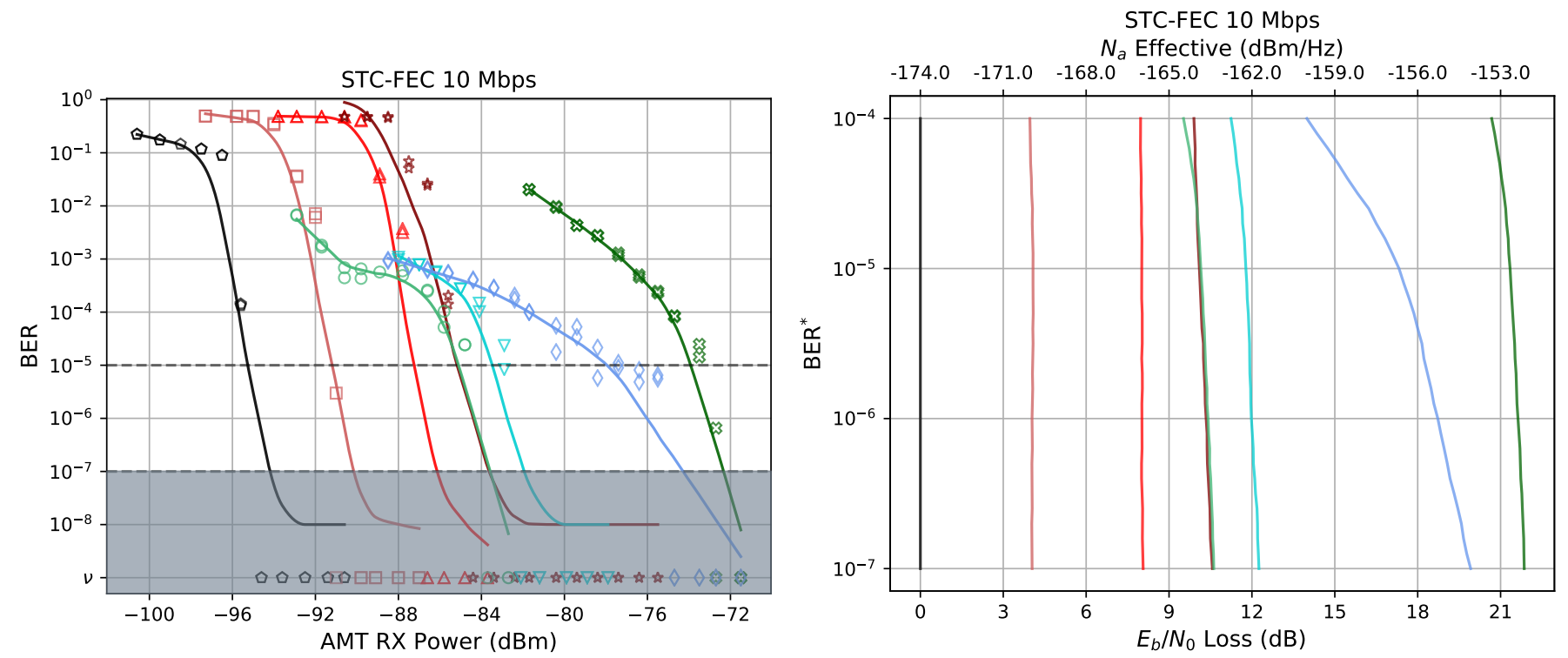

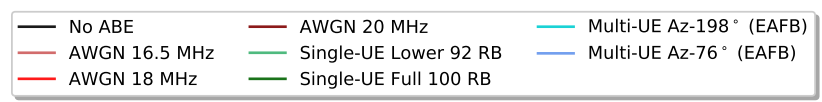

Figure 6.14: BER vs AMT RX power and BER* vs $N_{a}$ Effective for selected ABE types: STC-FEC $10 \mathrm{Mbps}$. The region $\mathrm{BER}<10^{-7}$ is shaded in the left plot to indicate high relative uncertainty. 


\subsection{Testbed Comparison}

The two testbeds were designed to share as many parts as feasible in their implementation. Notable differences are in the AMT TX and the VSG for ABE playback. Here we show the data products of the two testbeds side by side. The majority of results show a high degree of consistency in AMT RX reported susceptibility to the ABE. Though there were a few inconsistent results, these have been investigated and are shown to be repeatable. These outliers in response are likely attributable to inherent variances in the AMT RX implementation.

\subsubsection{Examples of Consistent Test Results}
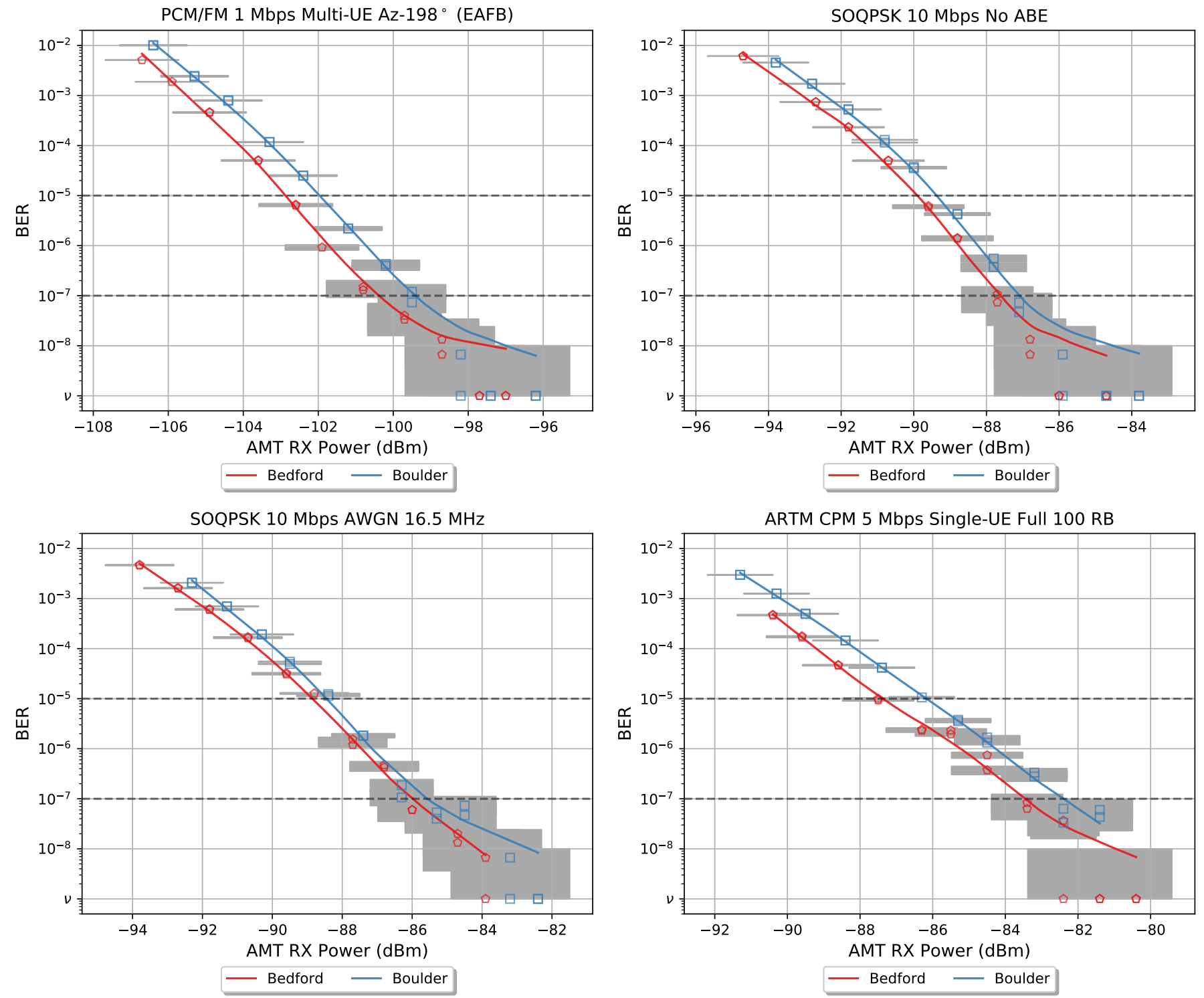

Figure 6.15: Examples showing consistent results between Bedford and Boulder testbeds. 


\subsubsection{Examples of Inconsistent Test Results}
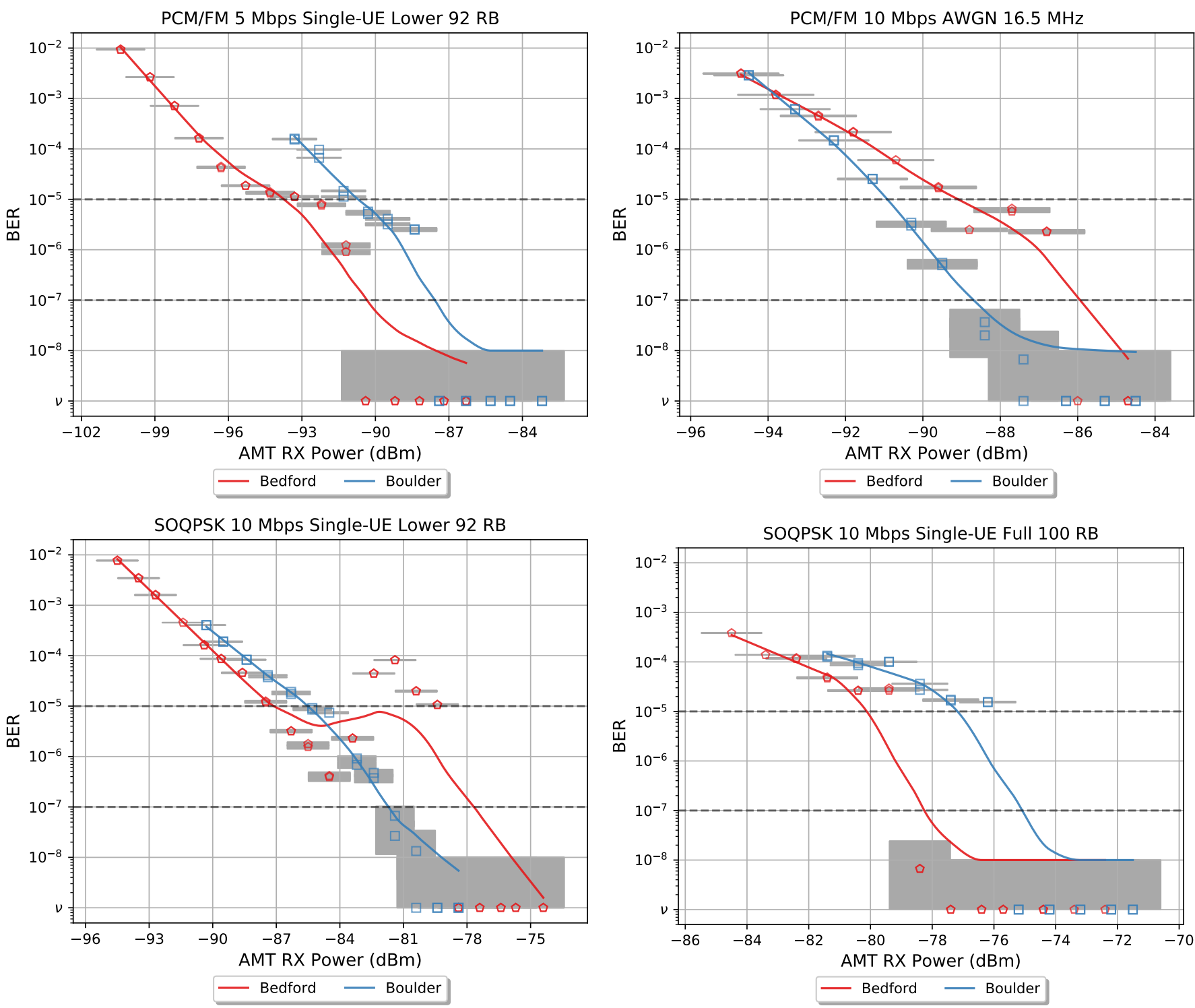

Figure 6.16: Examples showing inconsistent results between Bedford and Boulder testbeds. 


\section{Chapter 7}

\section{Conclusions}

The goal of NASCTN is to develop rigorous test methodologies and generate trusted data. To that end, some important observations from the test development and execution include the following:

- Proper consideration of OOBEs is important to understanding the impacts due to adjacent band signals. Currentlyavailable tools to simulate or emulate ABEs are not generally representative of real-world OOBEs, and those differences can have a significant impact on the adjacent band receiver.

- Automated testing allows for detailed and highly-controlled investigations of the impacts due to ABEs. In this case, the automation allows for the comparison of impacts from a wide variety of waveforms. Note that running a similar testing campaign in the field will, in general, result in higher measurement uncertainties than using a laboratory settings such as in this investigation.

- Two ABE waveforms with nearly equivalent average powers but with seemingly inconsequential differences in OOBE behavior can cause very different impacts on the AMT system. This is clearly evident in the frequency back-off results.

- The main experiment frequency back-off results suggest that the IRIG back-off calculations work for most of the test ABE types. In the main experiment results, the only ABE type and AMT modulation combinations that exceed a BER $=10^{-5}$ (i.e., exceed the desired threshold), are the multi-UE at azimuth of $198^{\circ}$ ( $37 \mathrm{MHz}$ filter) for SOQPSK bit rates of $5 \mathrm{Mbps}$ and $10 \mathrm{Mbps}$, and the multi-UE at azimuth of $198^{\circ}$ (37 MHz filter) and a single-UE with $100 \mathrm{RB}$ (37 MHz filter) for an ARTM CPM bit rate of 5 Mbps.

- For the single UE cases tested, the ABE OOBE profile correlates to the LTE RB allocations.

- The impacts of the ABEs are dominated by the frequency overlap of the IF filter bandwidth and the adjacent band LTE and LTE OOBE activity.

- The use of the ABE RMS power value in the SABE calculations can lead to misrepresentation in predicting impact of a particular waveform, when compared to the no ABE condition.

- As demonstrated in Appendix D scatter plots, receiver-reported $E_{b} / N_{0}$ is not a reliable indicator of BER performance in the presence of $\mathrm{ABE}$. Since $E_{b} / N_{0}$ is a ratio of in-band quantities, this observation is expected.

Beyond the specific test implementation and results discussed there are some identified topics that warrant further investigation that are listed here.

- The concept of an equivalent white ambient noise is proposed as a way to interpret the impacts on $E_{b} / N_{0}$. This may provided a convenient way to relate $\mathrm{ABE}$ impacts to theoretically based models. 
- The test results demonstrate non-linear effects across a range of BER values that fall in the desired operating region of better than BER $=10^{-5}$ for several of the tested ABE and AMT signal combinations. This suggests the need for further investigation on the approach of extrapolating performance behaviors based on higher BERs to lower BERs when the OOBE is non-Gaussian.

- The in-field measurements serve as surrogates and may not replicate the exact adjacent band deployment parameters (e.g., $20 \mathrm{MHz}$ versus $10 \mathrm{MHz}$ allocations, LTE versus $5 \mathrm{G}$ technology). In general, changes that allow increased RB activity or power at the band edge closest to AMT may require an increase in the band edge frequency offset. 


\section{Appendix A}

\section{Measurement Uncertainty}

This measurement campaign measures adjacent band emissions impact on an AMT link between an AMT TX and AMT RX. The AMT componentry used in this campaign is recognized as non-metrology grade equipment. Hence, conservatism in the assessment of uncertainties is warranted. To this end the authors make the following decisions in the uncertainty estimation:

- The signal level data presented in this document is referenced to the plane of the device under test (DUT) input connector.

- In the absence of carefully characterized transmitting elements and receiving DUT, we reference the signal and $\mathrm{ABE}$ power uncertainties at the testbed reference planes to a matched $50 \Omega$ load.

- We present expanded uncertainties in the $\mathrm{dB}$ scale by a factor of 2 multiplication of standard uncertainties, which is an overestimate of uncertainty due to non-linearity in log-space.

- We assume uncorrelated uncertainties and perform the conservative uncertainty budget calculations.

\section{A.1 AMT transmitter signal power at the AMT receiver input

$$
S_{R X}=S_{T X}-S_{P L}-A T_{1},
$$

Where $S_{R X}$ denotes the AMT signal power at the plane of the receiver input, $S_{T X}$ is the measured output power of the AMT transmitter at the face of the transmitter assembly, $S_{P L}$ the calibrated path loss of the testbed along the signal path at zero attenuation of the AMT signal-path attenuator, and $A T_{1}$ which is the calibrated value of the AMT signal path attenuator reported value. 
Table A.1: Power meter measurements at the face of the transmitter assembly. Note: LDPC waveforms were at code rate of $2 / 3$ and 4096 bits.

\begin{tabular}{|c|c|c|c|}
\hline Testbed & Modulation & Data Rate & Signal (dBm) \\
\hline Boulder & $\begin{array}{l}\mathrm{PCM} / \mathrm{FM} \\
\mathrm{PCM} / \mathrm{FM} \\
\mathrm{PCM} / \mathrm{FM} \\
\mathrm{PCM} / \mathrm{FM}\end{array}$ & $\begin{array}{l}1 \mathrm{Mbps} \\
5 \mathrm{Mbps} \\
10 \mathrm{Mbps} \\
20 \mathrm{Mbps}\end{array}$ & $\begin{array}{l}-1.6 \pm 0.3 \\
-1.6 \pm 0.3 \\
-1.5 \pm 0.3 \\
-1.5 \pm 0.3 \\
\end{array}$ \\
\hline Boulder & $\begin{array}{l}\text { SOQPSK } \\
\text { SOQPSK } \\
\text { SOQPSK } \\
\text { SOQPSK }\end{array}$ & $\begin{array}{l}1 \mathrm{Mbps} \\
5 \mathrm{Mbps} \\
10 \mathrm{Mbps} \\
20 \mathrm{Mbps}\end{array}$ & $\begin{array}{l}-1.6 \pm 0.3 \\
-1.6 \pm 0.3 \\
-1.6 \pm 0.3 \\
-1.5 \pm 0.3\end{array}$ \\
\hline Boulder & $\begin{array}{l}\text { ARTM CPM } \\
\text { ARTM CPM } \\
\text { ARTM CPM } \\
\end{array}$ & $\begin{array}{l}5 \mathrm{Mbps} \\
10 \mathrm{Mbps} \\
20 \mathrm{Mbps} \\
\end{array}$ & $\begin{array}{l}-1.6 \pm 0.3 \\
-1.5 \pm 0.3 \\
-1.5 \pm 0.3 \\
\end{array}$ \\
\hline Bedford & $\begin{array}{l}\mathrm{PCM} / \mathrm{FM} \\
\mathrm{PCM} / \mathrm{FM} \\
\mathrm{PCM} / \mathrm{FM} \\
\mathrm{PCM} / \mathrm{FM} \\
\end{array}$ & $\begin{array}{l}1 \mathrm{Mbps} \\
5 \mathrm{Mbps} \\
10 \mathrm{Mbps} \\
20 \mathrm{Mbps} \\
\end{array}$ & $\begin{array}{l}-4.6 \pm 0.3 \\
-4.6 \pm 0.3 \\
-4.6 \pm 0.3 \\
-4.6 \pm 0.3 \\
\end{array}$ \\
\hline Bedford & $\begin{array}{l}\text { SOQPSK } \\
\text { SOQPSK } \\
\text { SOQPSK } \\
\text { SOQPSK } \\
\end{array}$ & $\begin{array}{l}1 \mathrm{Mbps} \\
5 \mathrm{Mbps} \\
10 \mathrm{Mbps} \\
20 \mathrm{Mbps}\end{array}$ & $\begin{array}{l}-4.6 \pm 0.3 \\
-4.6 \pm 0.3 \\
-4.6 \pm 0.3 \\
-4.6 \pm 0.3\end{array}$ \\
\hline Bedford & $\begin{array}{l}\text { ARTM CPM } \\
\text { ARTM CPM } \\
\text { ARTM CPM } \\
\text { ARTM CPM }\end{array}$ & $\begin{array}{l}1 \mathrm{Mbps} \\
5 \mathrm{Mbps} \\
10 \mathrm{Mbps} \\
20 \mathrm{Mbps}\end{array}$ & $\begin{array}{l}-4.6 \pm 0.3 \\
-4.6 \pm 0.3 \\
-4.6 \pm 0.3 \\
-4.6 \pm 0.3 \\
\end{array}$ \\
\hline Bedford & $\begin{array}{l}\text { SOQPSK LDPC } \\
\text { SOQPSK LDPC } \\
\text { SOQPSK LDPC } \\
\text { SOQPSK LDPC }\end{array}$ & $\begin{array}{l}1 \mathrm{Mbps} \\
5 \mathrm{Mbps} \\
10 \mathrm{Mbps} \\
20 \mathrm{Mbps}\end{array}$ & $\begin{array}{l}-4.6 \pm 0.3 \\
-4.6 \pm 0.3 \\
-4.6 \pm 0.3 \\
-4.6 \pm 0.3\end{array}$ \\
\hline Bedford & $\begin{array}{l}\text { STC } \\
\text { STC } \\
\text { STC } \\
\text { STC }\end{array}$ & $\begin{array}{l}1 \mathrm{Mbps} \\
5 \mathrm{Mbps} \\
10 \mathrm{Mbps} \\
20 \mathrm{Mbps}\end{array}$ & $\begin{array}{l}-4.9 \pm 0.3 \\
-4.9 \pm 0.3 \\
-4.9 \pm 0.3 \\
-4.9 \pm 0.3 \\
\end{array}$ \\
\hline Bedford & $\begin{array}{l}\text { STC LDPC } \\
\text { STC LDPC } \\
\text { STC LDPC } \\
\end{array}$ & $\begin{array}{l}1 \mathrm{Mbps} \\
5 \mathrm{Mbps} \\
10 \mathrm{Mbps}\end{array}$ & $\begin{array}{l}-4.9 \pm 0.3 \\
-4.9 \pm 0.3 \\
-4.9 \pm 0.3 \\
\end{array}$ \\
\hline
\end{tabular}


Table A.2: Sources of uncertainty and uncertainty budget for the AMT signal power at the plane of the receiver at the Boulder Test Site.

\begin{tabular}{|c|c|c|c|c|c|c|c|}
\hline Classification & Factor & $\begin{array}{l}\text { Probability } \\
\text { Distribution }\end{array}$ & $\begin{array}{l}\text { Evaluation } \\
\text { Type }\end{array}$ & Designator & $\begin{array}{l}\text { Uncertainty } \\
\text { (dB) }\end{array}$ & $\begin{array}{l}\text { Correction for } \\
\text { Distribution }\end{array}$ & $\begin{array}{l}\text { Standard } \\
\text { Uncertainty (dB) }\end{array}$ \\
\hline \multirow{2}{*}{ Instrumentation } & Power Meter & Normal & $\mathrm{B}$ & $\mathrm{P} 1$ & 0.11 & 1 & 0.11 \\
\hline & Measurement standard deviation & Normal & A & PM1 & 0.10 & 1 & 0.10 \\
\hline \multirow{4}{*}{ Testbed } & Network Analyzer Calibration & Normal & $\mathrm{B}$ & $\mathrm{T} 1$ & 0.14 & 1 & 0.14 \\
\hline & Attenuator Calibration & Normal & $\mathrm{B}$ & $\mathrm{T} 2$ & 0.14 & 1 & 0.14 \\
\hline & Frequency Flatness & Normal & A & $\mathrm{T} 3$ & 0.07 & 1 & 0.07 \\
\hline & Mismatch \& Connector Repeat & Normal & $\mathrm{B}$ & $\mathrm{T} 4$ & 0.05 & 1 & 0.05 \\
\hline \multirow{2}{*}{ Total } & Combined standard uncertainty & & & & & & $0.45 \mathrm{~dB}$ \\
\hline & Expanded uncertainty $(k=2)$ & & & & & & $0.90 \mathrm{~dB}$ \\
\hline
\end{tabular}

Table A.3: Sources of uncertainty and uncertainty budget for the AMT signal power at the plane of the receiver at the Bedford Test Site.

\begin{tabular}{|c|c|c|c|c|c|c|c|}
\hline \multirow[t]{3}{*}{ Classification } & Factor & $\begin{array}{l}\text { Probability } \\
\text { Distribution }\end{array}$ & $\begin{array}{l}\text { Evaluation } \\
\text { Type }\end{array}$ & Designator & $\begin{array}{l}\text { Uncertainty } \\
\text { (dB) }\end{array}$ & $\begin{array}{l}\text { Correction for } \\
\text { Distribution }\end{array}$ & $\begin{array}{l}\text { Standard } \\
\text { Uncertainty (dB) }\end{array}$ \\
\hline & Power Meter & Normal & $\mathrm{B}$ & $\mathrm{P} 1$ & 0.15 & 1 & 0.15 \\
\hline & Measurement standard deviation & Normal & A & PM1 & 0.07 & 1 & 0.07 \\
\hline \multirow{4}{*}{ Testbed } & Network Analyzer Calibration & Normal & B & $\mathrm{T} 1$ & 0.14 & 1 & 0.14 \\
\hline & Attenuator Calibration & Normal & $\mathrm{B}$ & $\mathrm{T} 2$ & 0.14 & 1 & 0.14 \\
\hline & Frequency flatness & Normal & $\mathrm{A}$ & $\mathrm{T} 3$ & 0.11 & 1 & 0.11 \\
\hline & Mismatch \& Connector Repeat & Normal & $\mathrm{B}$ & $\mathrm{T} 4$ & 0.05 & 1 & 0.05 \\
\hline \multirow{2}{*}{ Total } & Combined standard uncertainty & & & & & & $0.49 \mathrm{~dB}$ \\
\hline & Expanded uncertainty $(k=2)$ & & & & & & $0.98 \mathrm{~dB}$ \\
\hline
\end{tabular}

Note, where the Expanded Uncertainty $(\mathrm{k}=2)$ for AMT signal power at the receiver is:

$$
2 * \sqrt{(P 1+P M 1)^{2}+(T 1+T 2+T 3+T 4)^{2}}
$$




\section{A.2 ABE power at the AMT receiver input}

$\mathrm{ABE}$ power at the plane of the receiver input is defined as the in-band power of the ABE signal. We make use of two descriptions of power, the peak power and the RMS power. Typical adjacent band power levels are described in terms of the RMS power internal to the ABE allocation. To make the assessment of the ABE power at the plane of the AMT receiver input, we characterize the power output of the VSG for the AWGN waveforms with a power meter $\left(A B E_{T X}\right)$, and subsequently take into account calibrated gain of the $\mathrm{ABE}$ path in the testbed $\left(A B E_{P L}\right)$, and the corresponding variable attenuator calibrated value $\left(A T_{2}\right)$.

$$
\left.A B E_{A W G N}\right|_{R X}=A B E_{T X}-A B E_{P L}-A T_{2}+C,
$$

where $C$ is a correction factor that takes into account the offset between calculated $A W G N$ waveform RMS dB relative to full scale (dBFS) and power meter measured output power.

Recorded structured waveforms such as the in-situ or lab captured LTE UL waveforms used for playback in this test were referenced to the calculated dBFS power from scaled versions of their IQ recordings.

$$
A B E_{R X}=A B E_{d B F S}-A B E_{P L}-A T_{2}+C
$$

The final, calibrated ABE power values and uncertainties for the two testbeds are given in Table A.6 and Table A.7. 
Table A.4: Sources of uncertainty and uncertainty budget for the ABE power at the plane of the receiver at the Boulder Test Site.

\begin{tabular}{|c|c|c|c|c|c|c|c|}
\hline Classification & Factor & $\begin{array}{l}\text { Probability } \\
\text { Distribution }\end{array}$ & $\begin{array}{l}\text { Evaluation } \\
\text { Type }\end{array}$ & Designator & $\begin{array}{l}\text { Uncertainty } \\
\text { (dB) }\end{array}$ & $\begin{array}{l}\text { Correction for } \\
\text { Distribution }\end{array}$ & $\begin{array}{l}\text { Standard } \\
\text { Uncertainty (dB) }\end{array}$ \\
\hline \multirow{3}{*}{\multicolumn{8}{|c|}{$\begin{array}{l}\text { VSG Level Error } \\
\text { VSG IQ Level Error } \\
\text { Instrumentation or }\end{array}$}} \\
\hline & & & & & & & \\
\hline & & & & & & & \\
\hline & Power Meter & Normal & $\mathrm{B}$ & $\mathrm{P} 1$ & 0.11 & 1 & 0.11 \\
\hline & Measurements standard deviation & Normal & A & PM2 & 0.08 & 1 & 0.08 \\
\hline \multirow{4}{*}{ Testbed } & Network Analyzer Calibration & Normal & $\mathrm{B}$ & $\mathrm{T} 1$ & 0.14 & 1 & 0.14 \\
\hline & Attenuator Calibration & Normal & $\mathrm{B}$ & $\mathrm{T} 2$ & 0.14 & 1 & 0.14 \\
\hline & Frequency flatness & Normal & A & T3 & 0.07 & 1 & 0.07 \\
\hline & Mismatch \& Connector Repeat & Normal & $\mathrm{B}$ & $\mathrm{T} 4$ & 0.05 & 1 & 0.05 \\
\hline \multirow{2}{*}{ Totall $_{\text {AWGN }}$} & Combined standard uncertainty & & & & & & $0.44 \mathrm{~dB}$ \\
\hline & Expanded uncertainty $(k=2)$ & & & & & & $0.89 \mathrm{~dB}$ \\
\hline \multirow{2}{*}{ Totall $_{\text {LTE }}$} & Combined standard uncertainty & & & & & & $0.57 \mathrm{~dB}$ \\
\hline & Expanded uncertainty $(k=2)$ & & & & & & $1.13 \mathrm{~dB}$ \\
\hline
\end{tabular}

Note, where the Expanded Uncertainty $(\mathrm{k}=2)$ for ABE power at the plane of the receiver of AWGN waveforms is,

$$
2 * \sqrt{(P 1+P M 2)^{2}+(T 1+T 2+T 3+T 4)^{2}}
$$

and for the structured IQ waveforms it is:

$$
2 * \sqrt{(V 1+V 2)^{2}+(T 1+T 2+T 3+T 4)^{2}}
$$


Table A.5: Sources of uncertainty and uncertainty budget for the ABE power at the plane of the receiver at the Bedford Test Site.

\begin{tabular}{|c|c|c|c|c|c|c|c|}
\hline Classification & Factor & $\begin{array}{l}\text { Probability } \\
\text { Distribution }\end{array}$ & $\begin{array}{l}\text { Evaluation } \\
\text { Type }\end{array}$ & Designator & $\begin{array}{l}\text { Uncertainty } \\
\text { (dB) }\end{array}$ & $\begin{array}{l}\text { Correction for } \\
\text { Distribution }\end{array}$ & $\begin{array}{l}\text { Standard } \\
\text { Uncertainty }\end{array}$ \\
\hline \multirow[t]{3}{*}{ Instrumentatio } & $\begin{array}{l}\text { VSG Level Error } \\
\text { or }\end{array}$ & Normal & $\mathrm{B}$ & V1 & 0.40 & 1 & 0.40 \\
\hline & Power Meter & Normal & $\mathrm{B}$ & $\mathrm{P} 1$ & 0.15 & 1 & 0.15 \\
\hline & Measurements standard deviation & Normal & A & PM2 & 0.11 & 1 & 0.11 \\
\hline \multirow{4}{*}{ Testbed } & Network Analyzer Calibration & Normal & $\mathrm{B}$ & $\mathrm{T} 1$ & 0.14 & 1 & 0.14 \\
\hline & Attenuator Calibration & Normal & $\mathrm{B}$ & $\mathrm{T} 2$ & 0.14 & 1 & 0.14 \\
\hline & Frequency flatness & Normal & A & $\mathrm{T} 3$ & 0.11 & 1 & 0.07 \\
\hline & Mismatch \& Connector Repeat & Normal & $\mathrm{B}$ & $\mathrm{T} 4$ & 0.05 & 1 & 0.05 \\
\hline Totall $_{\mathrm{AWGN}}$ & $\begin{array}{l}\text { Combined standard uncertainty } \\
\text { Expanded uncertainty }(\mathbf{k = 2})\end{array}$ & & & & & & $\begin{array}{l}0.51 \\
1.02 \mathrm{~dB}\end{array}$ \\
\hline Totall $_{\text {LTE }}$ & $\begin{array}{l}\text { Combined standard uncertainty } \\
\text { Expanded uncertainty }(\mathbf{k}=\mathbf{2})\end{array}$ & & & & & & $\begin{array}{l}0.59 \mathrm{~dB} \\
\mathbf{1 . 1 9} \mathrm{dB}\end{array}$ \\
\hline
\end{tabular}

Table A.6: ABE Power level table, Main Experiment campaign conducted at the MITRE Bedford, MA testbed and NASCTN Boulder, CO testbed.

\begin{tabular}{|l|c|c|c|c|}
\hline & \multicolumn{2}{|c|}{ MITRE Bedford, MA } & \multicolumn{2}{c|}{ NASCTN Boulder, CO } \\
Waveform Name & Average Power & Peak Power & Average Power & Peak Power \\
\hline AWGN 20 MHz & $-75.8 \mathrm{dBm} \pm 1.0 \mathrm{~dB}$ & N/A & $-75.6 \mathrm{dBm} \pm 0.9 \mathrm{~dB}$ & N/A \\
AWGN 18 MHz & $-76.4 \mathrm{dBm} \pm 1.0 \mathrm{~dB}$ & N/A & $-76.1 \mathrm{dBm} \pm 0.9 \mathrm{~dB}$ & N/A \\
AWGN 16.5 MHz & $-76.8 \mathrm{dBm} \pm 1.0 \mathrm{~dB}$ & N/A & $-76.5 \mathrm{dBm} \pm 0.9 \mathrm{~dB}$ & N/A \\
\hline Single-UE Full 100 RB (EAFB, 20 MHz Filter) & $-69.9 \mathrm{dBm} \pm 1.2 \mathrm{~dB}$ & $-61.8 \mathrm{dBm} \pm 1.2 \mathrm{~dB}$ & $-69.6 \mathrm{dBm} \pm 1.1 \mathrm{~dB}$ & $-61.6 \mathrm{dBm} \pm 1.1 \mathrm{~dB}$ \\
Single-UE Lower 92 RB (EAFB, 20 MHz Filter) & $-72.8 \mathrm{dBm} \pm 1.2 \mathrm{~dB}$ & $-62.3 \mathrm{dBm} \pm 1.2 \mathrm{~dB}$ & $-72.6 \mathrm{dBm} \pm 1.1 \mathrm{~dB}$ & $-62.0 \mathrm{dBm} \pm 1.1 \mathrm{~dB}$ \\
\hline Multi-UE Az-76 100 RB (EAFB, 20 MHz Filter) & $-84.6 \mathrm{dBm} \pm 1.2 \mathrm{~dB}$ & $-62.1 \mathrm{dBm} \pm 1.2 \mathrm{~dB}$ & $-84.4 \mathrm{dBm} \pm 1.1 \mathrm{~dB}$ & $-61.8 \mathrm{dBm} \pm 1.1 \mathrm{~dB}$ \\
Multi-UE Az-198 $100 \mathrm{RB}$ (EAFB, 20 MHz Filter) & $-86.5 \mathrm{dBm} \pm 1.2 \mathrm{~dB}$ & $-62.1 \mathrm{dBm} \pm 1.2 \mathrm{~dB}$ & $-86.3 \mathrm{dBm} \pm 1.1 \mathrm{~dB}$ & $-61.8 \mathrm{dBm} \pm 1.1 \mathrm{~dB}$ \\
Multi-UE Az-140 $100 \mathrm{RB}$ (LARC, 20 MHz Filter) & $-80.3 \mathrm{dBm} \pm 1.2 \mathrm{~dB}$ & $-62.1 \mathrm{dBm} \pm 1.2 \mathrm{~dB}$ & $-80.0 \mathrm{dBm} \pm 1.1 \mathrm{~dB}$ & $-61.8 \mathrm{dBm} \pm 1.1 \mathrm{~dB}$ \\
Mult-UE Az-165 $100 \mathrm{RB}$ (LARC, 20 MHz Filter) & $-78.9 \mathrm{dBm} \pm 1.2 \mathrm{~dB}$ & $-62.1 \mathrm{dBm} \pm 1.2 \mathrm{~dB}$ & $-78.6 \mathrm{dBm} \pm 1.1 \mathrm{~dB}$ & $-61.8 \mathrm{dBm} \pm 1.1 \mathrm{~dB}$ \\
\hline
\end{tabular}


Table A.7: ABE Power level table, for the Frequency Offset Experiment conducted at the MITRE Bedford, MA testbed and NASCTN Boulder, CO testbed.

\begin{tabular}{|l|c|c|c|c|}
\hline & \multicolumn{2}{|c|}{ MITRE Bedford, MA } & \multicolumn{2}{c|}{ NASCTN Boulder, CO } \\
Waveform Name & Average Power & Peak Power & Average Power & Peak Power \\
\hline AWGN 20 MHz & $-59.6 \mathrm{dBm} \pm 1.0 \mathrm{~dB}$ & N/A & $-60.0 \mathrm{dBm} \pm 0.8 \mathrm{~dB}$ & N/A \\
\hline Single-UE Full 100 RB (EAFB, 20 MHz Filter) & $-60.5 \mathrm{dBm} \pm 1.2 \mathrm{~dB}$ & $-52.2 \mathrm{dBm} \pm 1.2 \mathrm{~dB}$ & $-60.5 \mathrm{dBm} \pm 1.1 \mathrm{~dB}$ & $-52.2 \mathrm{dBm} \pm 1.1 \mathrm{~dB}$ \\
Single-UE Lower 92 RB (EAFB, 20 MHz Filter) & $-63.5 \mathrm{dBm} \pm 1.2 \mathrm{~dB}$ & $-52.7 \mathrm{dBm} \pm 1.2 \mathrm{~dB}$ & $-63.5 \mathrm{dBm} \pm 1.1 \mathrm{~dB}$ & $-52.7 \mathrm{dBm} \pm 1.1 \mathrm{~dB}$ \\
Single-UE Full 100 RB (EAFB, 37 MHz Filter) & $-60.5 \mathrm{dBm} \pm 1.2 \mathrm{~dB}$ & $-52.4 \mathrm{dBm} \pm 1.2 \mathrm{~dB}$ & $-60.5 \mathrm{dBm} \pm 1.1 \mathrm{~dB}$ & $-52.4 \mathrm{dBm} \pm 1.1 \mathrm{~dB}$ \\
Single-UE Lower 92 RB (EAFB, 37 MHz Filter) & $-63.4 \mathrm{dBm} \pm 1.2 \mathrm{~dB}$ & $-52.8 \mathrm{dBm} \pm 1.2 \mathrm{~dB}$ & $-63.5 \mathrm{dBm} \pm 1.1 \mathrm{~dB}$ & $-52.8 \mathrm{dBm} \pm 1.1 \mathrm{~dB}$ \\
\hline Multi-UE Az-76 ${ }^{\circ}$ 100 RB (EAFB, 37 MHz Filter) & $-70.6 \mathrm{dBm} \pm 1.2 \mathrm{~dB}$ & $-48.0 \mathrm{dBm} \pm 1.2 \mathrm{~dB}$ & $-70.5 \mathrm{dBm} \pm 1.1 \mathrm{~dB}$ & $-47.9 \mathrm{dBm} \pm 1.1 \mathrm{~dB}$ \\
Multi-UE Az-198 100 RB (EAFB, 37 MHz Filter) & $-82.5 \mathrm{dBm} \pm 1.2 \mathrm{~dB}$ & $-53.9 \mathrm{dBm} \pm 1.2 \mathrm{~dB}$ & $-82.3 \mathrm{dBm} \pm 1.1 \mathrm{~dB}$ & $-53.8 \mathrm{dBm} \pm 1.1 \mathrm{~dB}$ \\
\hline Single-UE Full 100 RB (Lab, 49 MHz Filter) & $-66.0 \mathrm{dBm} \pm 1.2 \mathrm{~dB}$ & $-52.9 \mathrm{dBm} \pm 1.2 \mathrm{~dB}$ & $-65.4 \mathrm{dBm} \pm 1.1 \mathrm{~dB}$ & $-52.3 \mathrm{dBm} \pm 1.1 \mathrm{~dB}$ \\
Single-UE Lower 90 RB (Lab, 49 MHz Filter) & $-65.4 \mathrm{dBm} \pm 1.2 \mathrm{~dB}$ & $-52.9 \mathrm{dBm} \pm 1.2 \mathrm{~dB}$ & $-64.8 \mathrm{dBm} \pm 1.1 \mathrm{~dB}$ & $-52.3 \mathrm{dBm} \pm 1.1 \mathrm{~dB}$ \\
Single-UE Full 25 RB (Lab, 49 MHz Filter) & $-70.7 \mathrm{dBm} \pm 1.2 \mathrm{~dB}$ & $-52.9 \mathrm{dBm} \pm 1.2 \mathrm{~dB}$ & $-70.0 \mathrm{dBm} \pm 1.1 \mathrm{~dB}$ & $-52.2 \mathrm{dBm} \pm 1.1 \mathrm{~dB}$ \\
\hline
\end{tabular}




\section{A.3 Noise at the AMT receiver input}

We define here the derivation for the noise due to the test noise diode at the input connector of the RX. Lowercase symbols denote power-scaled quantities in linear units, whereas upper case symbols denote units in decibel.

The noise calibration procedure is referenced against a calibrated noise diode. In the first step, this reference noise diode is attached to the input connector of the VSA. This test configuration makes the noise power available to the instrument equal to

$$
\left.n_{\mathrm{VSA}}\right|_{1}=k_{\mathrm{B}} t_{D} b+k_{\mathrm{B}} t_{a} b
$$

where $t_{D}$ is the excess noise temperature of the reference noise diode, $t_{a}$ is the ambient physical temperature of the measurement apparatus, $k_{\mathrm{B}} \approx 1.38 \times 10^{-35} \mathrm{~mW} / \mathrm{Hz} / \mathrm{K}$ is Boltzmann's constant, and $b$ is the bandwidth of interest.

The excess noise temperature of the reference diode can be determined from the excess noise ratio (ENR) specified by its manufacturer. This is specified in decibels relative to a reference temperature, which is conventionally $t_{0}=290 \mathrm{~K}$ [27],

$$
\mathrm{ENR}=10 \log _{10}\left(\frac{t_{D}}{t_{0}}\right) .
$$

This, together with effective diode noise temperature defined as,

$$
t_{D e}=t_{0}\left(\frac{t_{a}}{t_{0}}+10^{\mathrm{ENR} / 10}\right)=t_{0}\left(\frac{t_{a}}{t_{0}}+\mathrm{enr}\right)
$$

simplifies (A.7) to

$$
\left.n_{\mathrm{VSA}}\right|_{1}=k_{\mathrm{B}} t_{D e} b .
$$

The second measurement of the calibration procedure adjusts the attenuation of the testbed noise path to match the VSA power reading to that of the reference diode. These readings include different external noise conditions, but the same gain and added noise characteristics of the VSA. At this balance point, the power gain between the testbed and the VSA is defined as $g_{c}$.

The noise output by the testbed is a lossy microwave network driven by a noise source. The fraction of incident power lost to dissipative heat in the lossy network is $l$. The output noise power $n_{\text {out }}$ is then

$$
n_{\text {out }}=(1-l) k_{\mathrm{B}} t_{S} b+l k_{\mathrm{B}} t_{a} b,
$$

where $t_{S}$ is the temperature of the noise source inside the testbed.

In this second calibration measurement, $g_{c}=1-l_{c}$, and $t_{S} \rightarrow t_{T}$ is the noise temperature of the source inside the testbed. Hence, we find that

$$
\left.n_{\mathrm{VSA}}\right|_{2}=g_{c} k_{\mathrm{B}} t_{T} b+\left(1-g_{c}\right) k_{\mathrm{B}} t_{a} b
$$

The noise calibration measurement procedure balances the VSA input noise levels as

$$
\left.n_{\mathrm{VSA}}\right|_{2}=\left.n_{\mathrm{VSA}}\right|_{1} .
$$


The output noise temperature of the testbed is therefore

$$
\begin{aligned}
g_{c} k_{\mathrm{B}} t_{T} b+\left(1-g_{c}\right) k_{\mathrm{B}} t_{a} b & =k_{\mathrm{B}} t_{D e} b, \\
g_{c} t_{T}+\left(1-g_{c}\right) t_{a} & =t_{D e}, \\
t_{T} & =\frac{1}{g_{c}}\left(t_{D e}-\left(1-g_{c}\right) t_{a}\right) .
\end{aligned}
$$

Substituting A.9 into A.14 yields

$$
\begin{aligned}
t_{T} & =\frac{1}{g_{c}}\left(\left(\frac{t_{a}}{t_{0}}+e n r\right) t_{0}-\left(1-g_{c}\right) t_{a}\right), \\
t_{T} & =t_{a}+t_{0} \frac{e n r}{g_{c}}
\end{aligned}
$$

To estimate the noise at the input connector of the receiver under test at an arbitrary testbed insertion loss $l_{T}$, we combine A.15, A.11, and $g_{T}=\left(1-l_{T}\right)$ to arrive at:

$$
\begin{aligned}
& N_{R x}=\left(1-l_{T}\right) k_{\mathrm{B}} t_{T} b+l_{T} k_{\mathrm{B}} t_{a} b \\
& N_{R x}=g_{T} k_{\mathrm{B}}\left(t_{a}+t_{0} \frac{e n r}{g_{c}}\right) b+\left(1-g_{T}\right) k_{\mathrm{B}} t_{a} b \\
& N_{R x}=k_{B} t_{0} b\left(\frac{t_{a}}{t_{0}}+\frac{g_{T}}{g_{c}} e n r\right) .
\end{aligned}
$$

Performing the measurement in a controlled indoor laboratory sets $t_{a} / t_{0} \approx 1$. Since $\frac{g_{T}}{g_{c}}$ enr $\gg 1$, then at standardized $t_{0}=290 \mathrm{~K}$, there is a negligibly small error in estimating $N_{R x}$ in decibel units as:

$$
N_{R x} \approx-174.0 \mathrm{dBm} / \mathrm{Hz}+\mathrm{ENR}+G_{T}-G_{c} .
$$

We decompose the gain through the testbed path, $G_{T}$, into a fixed minimum path loss (PL) at $0 \mathrm{~dB}$ variable attenuation and added variable attenuation, with calibrated value $A_{T}$, so that $G_{T}=-\left(\mathrm{PL}+A_{T}\right)$. At the calibrated variable attenuation $A_{T} \rightarrow A_{c}$ that balances the testbed output noise and the reference diode, the total testbed gain is $G_{c}=$ $-\left(\mathrm{PL}+A_{c}\right)$. In these terms, A.17 reduces further to

$$
\begin{aligned}
& N_{R x} \approx-174.0 \mathrm{dBm} / \mathrm{Hz}+\mathrm{ENR}+G_{T}-G_{c} \\
& N_{R x} \approx-174.0 \mathrm{dBm} / \mathrm{Hz}+\mathrm{ENR}-\mathrm{PL}-A_{T}-G_{c} .
\end{aligned}
$$

Specifically for the Boulder testbed we can fill in the values in A.18 with

- the ENR of the reference diode is $14.7 \mathrm{~dB}$,

- unattenuated path loss was measured as PL $=29.0 \mathrm{~dB}$,

- the gain at the calibration point measured $G_{c}=-68.7 \mathrm{~dB}$. 
The itemized standard uncertainty components are tabulated in A.8.

$$
\begin{aligned}
& \left.N_{R x}\right|_{\text {Boulder }}=-174 \mathrm{dBm} / \mathrm{Hz}+B-A_{T}+14.7 \mathrm{~dB}-29.0 \mathrm{~dB}+68.7 \mathrm{~dB} \\
& \left.N_{R x}\right|_{\text {Boulder }}=-119.6 \mathrm{dBm} / \mathrm{Hz}+B-A_{T}
\end{aligned}
$$


Table A.8: Sources of uncertainty and uncertainty budget for applied incident noise power at the plane of the receiver at the Boulder Test Site.

\begin{tabular}{|c|c|c|c|c|c|c|c|}
\hline Classification & Factor & $\begin{array}{l}\text { Probability } \\
\text { Distribution }\end{array}$ & $\begin{array}{l}\text { Evaluation } \\
\text { Type }\end{array}$ & Designator & $\begin{array}{l}\text { Uncertainty } \\
\text { (dB) }\end{array}$ & $\begin{array}{l}\text { Correction for } \\
\text { Distribution }\end{array}$ & $\begin{array}{l}\text { Standard } \\
\text { Uncertainty (dB) }\end{array}$ \\
\hline \multirow{2}{*}{ Instrumentation } & VSA measurement standard deviation (STD) & Normal & A & VSA1 & 0.14 & 1 & 0.14 \\
\hline & Reference noise diode ENR & Normal & $\mathrm{B}$ & ENR1 & 0.15 & 1 & 0.15 \\
\hline \multirow{4}{*}{ Testbed } & Network analyzer measurement & Normal & $\mathrm{B}$ & $\mathrm{T} 1$ & 0.14 & 1 & 0.14 \\
\hline & Attenuator calibration & Normal & $\mathrm{B}$ & $\mathrm{T} 2$ & 0.14 & 1 & 0.14 \\
\hline & Frequency flatness & Normal & A & T3 & 0.07 & 1 & 0.07 \\
\hline & Mismatch \& Connector Repeat & Normal & $\mathrm{B}$ & $\mathrm{T} 4$ & 0.05 & 1 & 0.05 \\
\hline \multirow{2}{*}{$\begin{array}{l}\text { Noise diode } \\
\text { calibration }\end{array}$} & Level offset $\left(A_{c}\right)$ & Normal & A & T5 & 0.35 & 1 & 0.35 \\
\hline & Network analyzer measurement & Normal & $\mathrm{B}$ & $\mathrm{T} 1$ & 0.14 & 1 & 0.14 \\
\hline Environmental & Temperature & Normal & $\mathrm{B}$ & E1 & 0.08 & 1 & 0.08 \\
\hline \multirow{2}{*}{ Total } & Combined standard uncertainty & & & & & & $0.92 \mathrm{~dB}$ \\
\hline & Expanded uncertainty $(k=2)$ & & & & & & $1.83 \mathrm{~dB}$ \\
\hline
\end{tabular}

N The expanded uncertainty is calculated as follows:

$$
2 * \sqrt{V S A 1^{2}+E N R 1^{2}+(2 * T 1+T 2+T 3+T 4+T 5)^{2}+E 1^{2}}
$$




\section{A.4 Uncertainty for ratio quantities}

\section{A.4.1 Eb/No}

The expanded uncertainty for $E_{b} / N_{0}$ calculations in the case of applied excess noise in the measurement system, where the excess noise is an experimental factor:

$$
2 * \sqrt{(P 1+P M 1)^{2}+V S A 1^{2}+E N R 1^{2}+(3 * T 1+2 * T 2+2 * T 3+2 * T 4+T 5)^{2}+E 1^{2}}
$$

In the case that $E_{b} / N_{0}$ calculations are performed without added excess noise, the expanded uncertainty reduces to

$$
2 * \sqrt{(P 1+P M 1)^{2}+(T 1+T 2+T 3+T 4)^{2}+E 1^{2}}
$$

\section{A.4.2 S/ABE}

The signal power here is defined as the AMT Power at the plane of the receiver $\left(S_{R M S}\right)$ and the ABE is defined as either $A B E_{A W G N}$ or $A B E_{L T E}$, where the various uncertainty factors are respective to the testbeds and waveform types.

The expanded uncertainty for the SABE is derived below.

$$
\begin{aligned}
S /\left.A B E\right|_{L T E} & =2 * \sqrt{(P 1+P M 1)^{2}+(V S G 1+V S G 2)^{2}+(2 * T 1+2 * T 2+2 * T 3+2 * T 4)^{2}} \\
S /\left.A B E\right|_{A W G N} & =2 * \sqrt{(2 * P 1+P M 1+P M 2)^{2}+(2 * T 1+2 * T 2+2 * T 3+2 * T 4)^{2}}
\end{aligned}
$$

\section{A.5 Summary of Uncertainty Values}

Table A.9: Summary of expanded uncertainty values for the various test conditions referenced to the N-type RF input connector of the AMT receiver

\begin{tabular}{l|l|ll|l}
$\begin{array}{l}\text { Testbed } \\
\text { Location }\end{array}$ & AMT Signal Power at RX & \multicolumn{2}{|c|}{ ABE Signal Power at Rx } & SABE at RX \\
Uncert. (dB) & ABE Type & Uncert. (dB) & Uncert. (dB) \\
\hline \multirow{2}{*}{ Bedford } & \multirow{2}{*}{ \pm 0.98} & AWGN & \pm 1.02 & \pm 2.00 \\
& & LTE & \pm 1.19 & \pm 1.98 \\
\hline \multirow{2}{*}{ Boulder } & \multirow{2}{*}{ \pm 0.90} & AWGN & \pm 0.89 & \pm 1.79 \\
& & LTE & \pm 1.13 & \pm 1.84 \\
\hline \hline
\end{tabular}

Table A.10: Summary of expanded uncertainty values for the various test conditions referenced to the N-type RF input connector of the AMT receiver

\begin{tabular}{c|ll} 
Testbed & \multicolumn{2}{|c}{ EbN0 at RX } \\
Location & Applied Noise & Uncert. (dB) \\
\hline Bedford & No & \pm 0.91 \\
\hline \multirow{2}{*}{ Boulder } & No & \pm 0.83 \\
& Yes & \pm 2.65 \\
\hline \hline
\end{tabular}




\section{Appendix B}

\section{Individual Test Plots for Main Experiment}
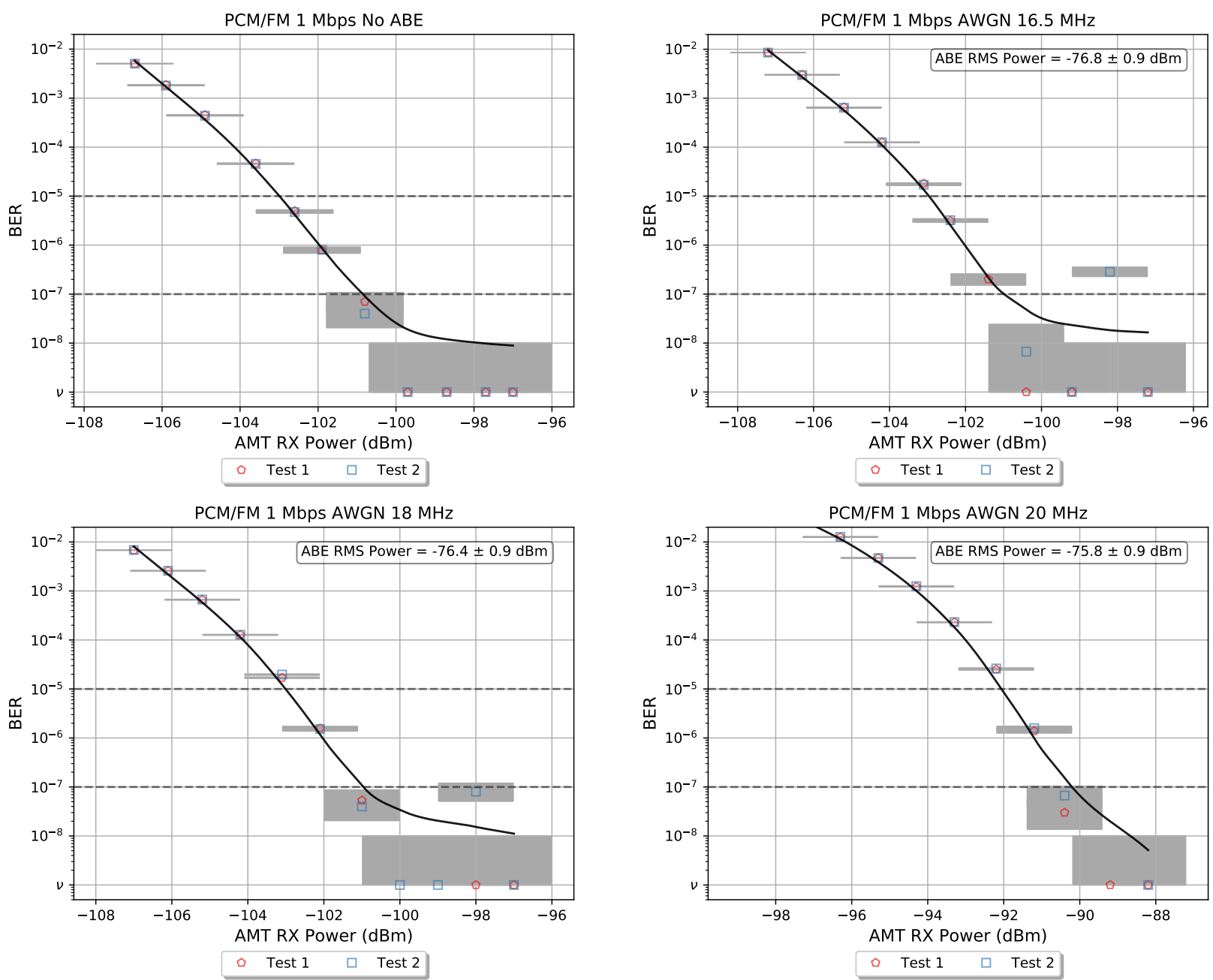

Figure B.1: Main Experiment configurations of PCM/FM 1 Mbps with No ABE and AWGN ABE. 

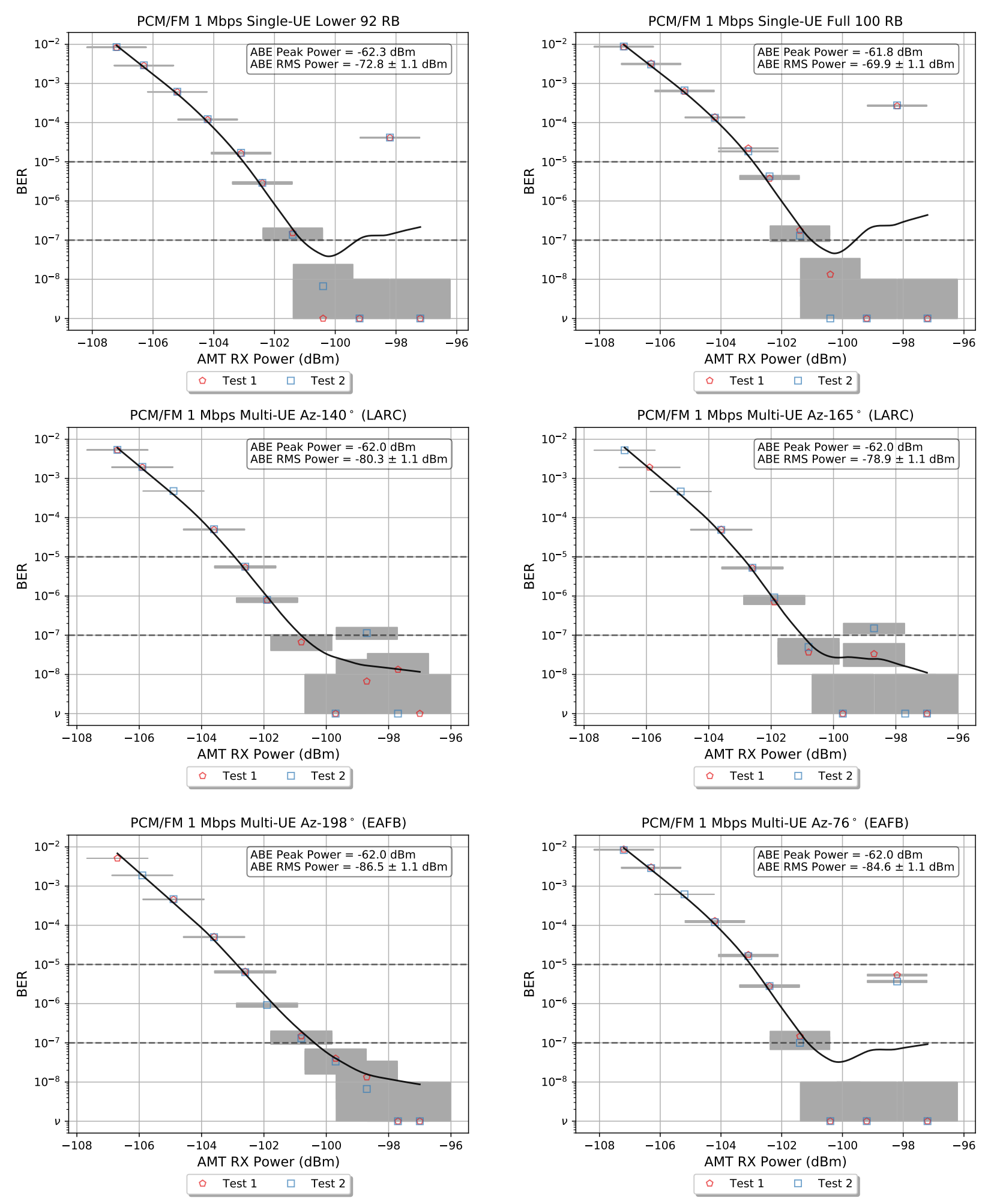

Figure B.2: Main Experiment configurations of PCM/FM 1 Mbps with single-UE and multi-UE LTE ABE. 

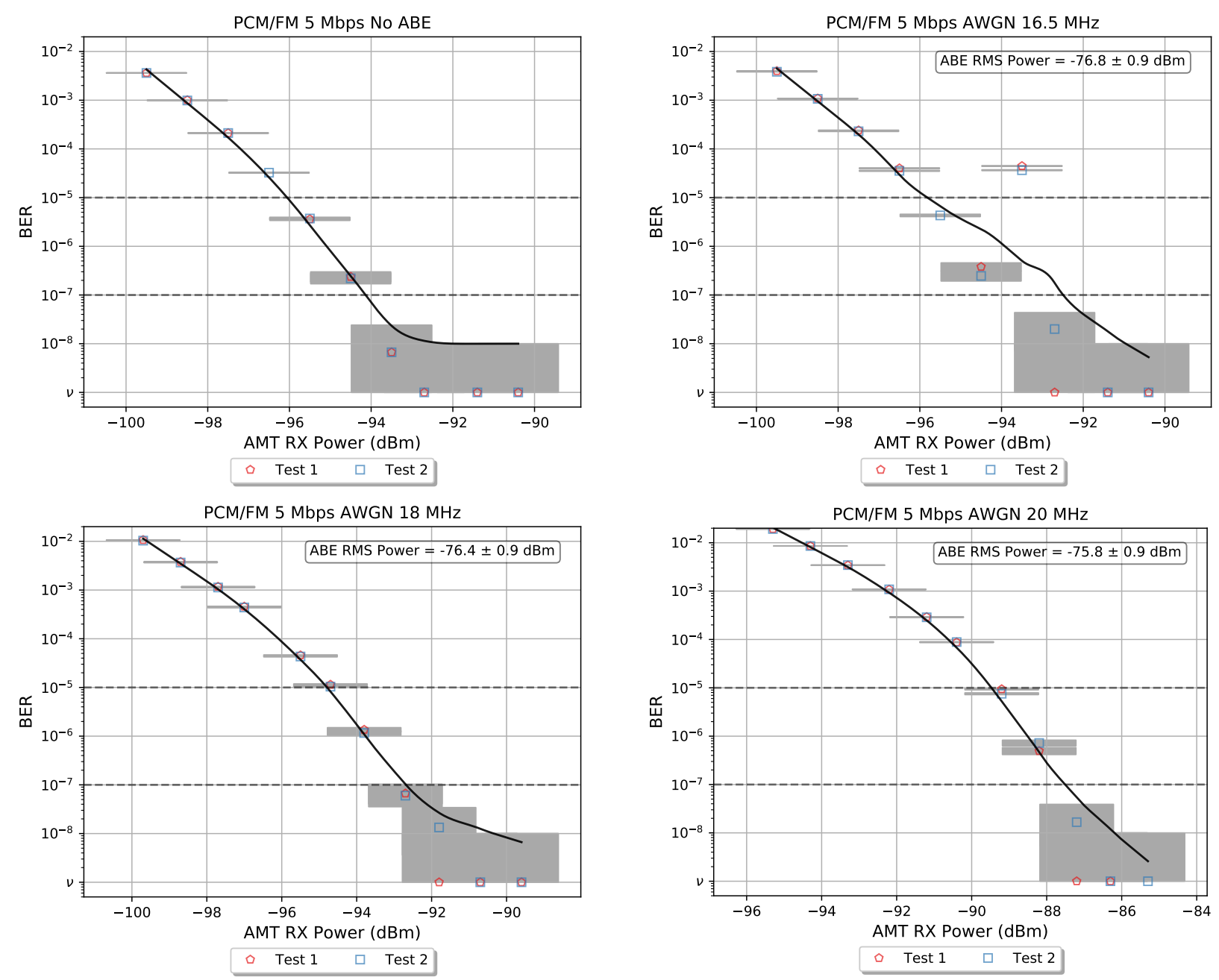

Figure B.3: Main Experiment configurations of PCM/FM 5 Mbps with No ABE and AWGN ABE. 

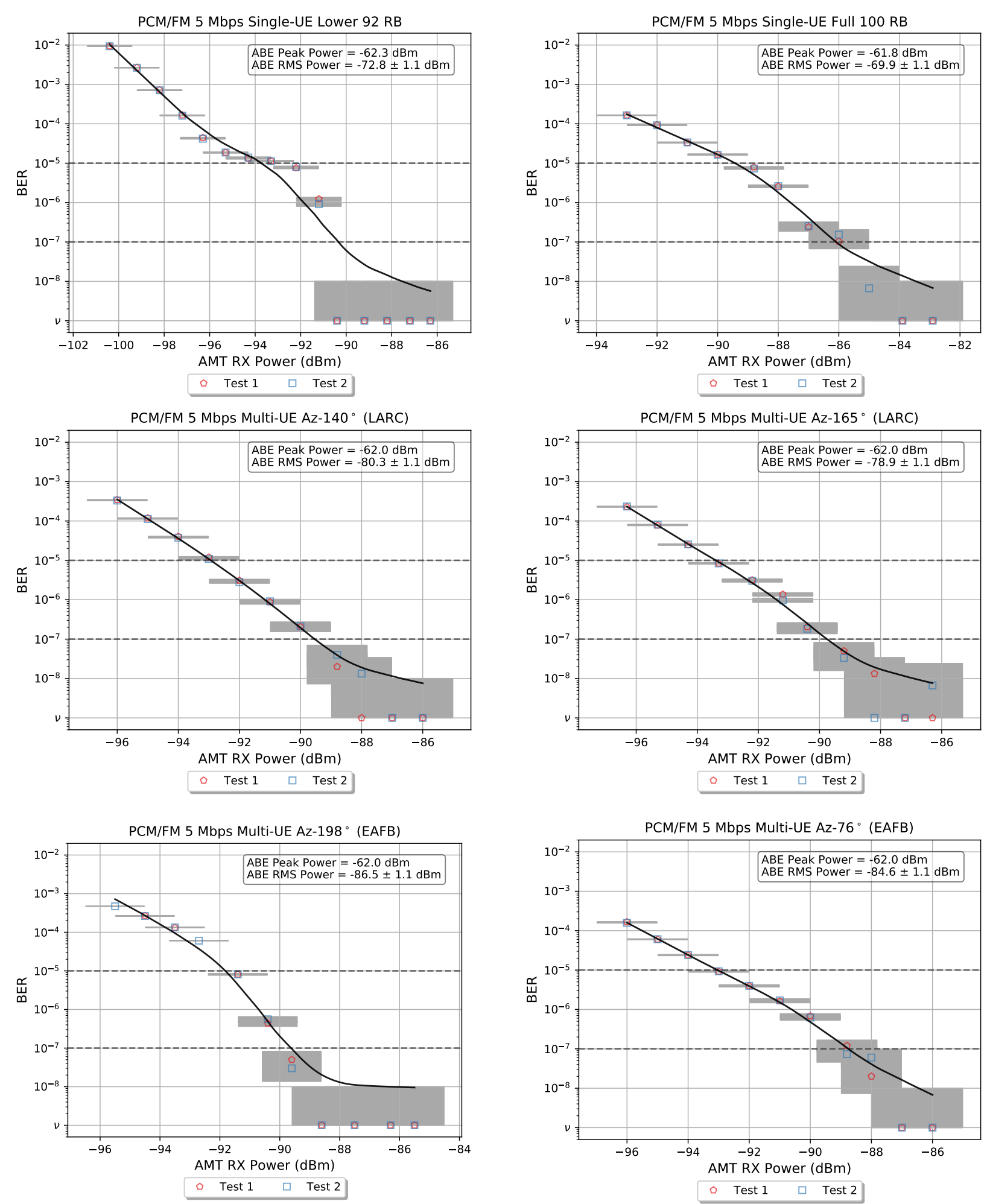

Figure B.4: Main Experiment configurations of PCM/FM 5 Mbps with single-UE and multi-UE LTE ABE. 

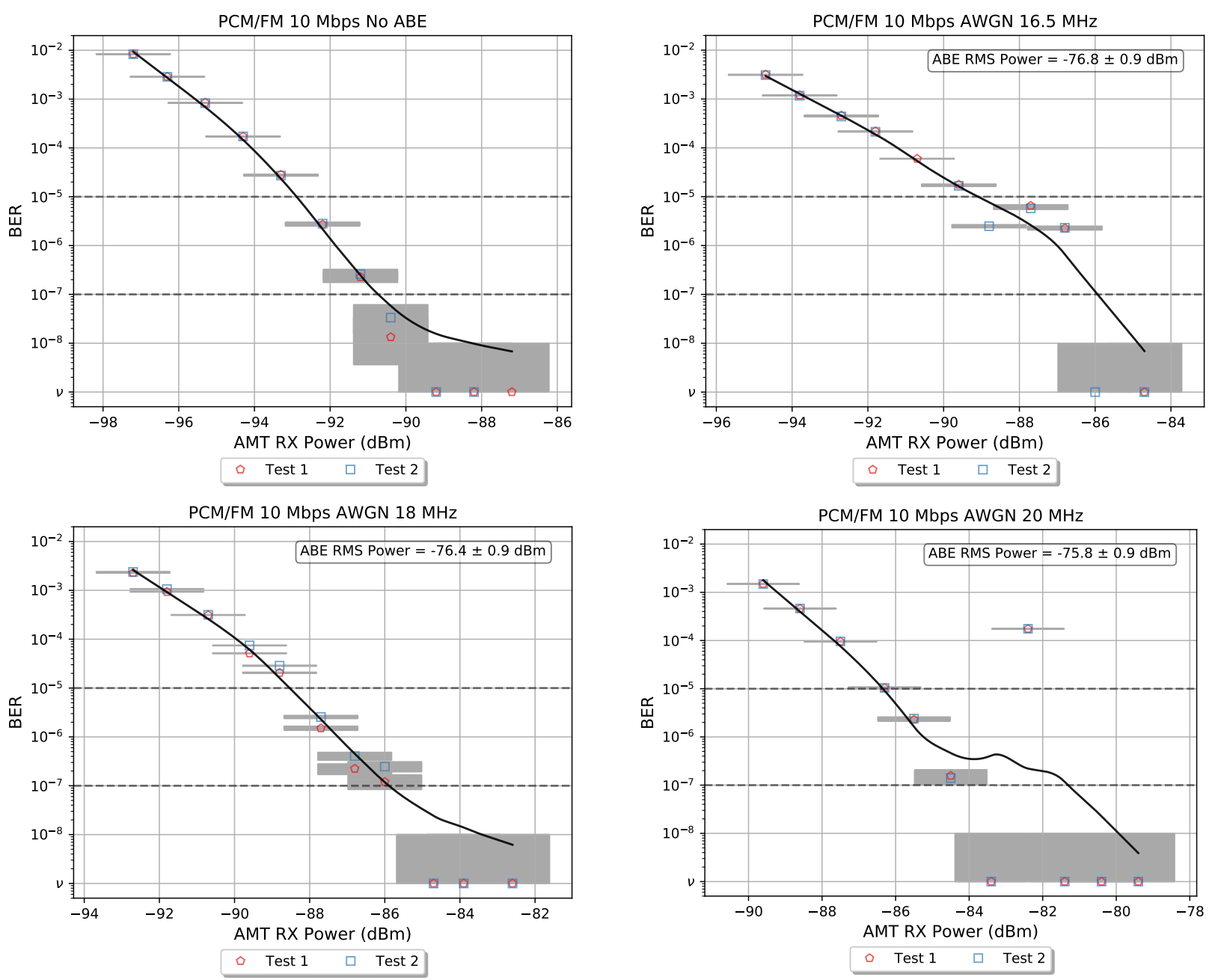

Figure B.5: Main Experiment configurations of PCM/FM 10 Mbps with No ABE and AWGN ABE. 

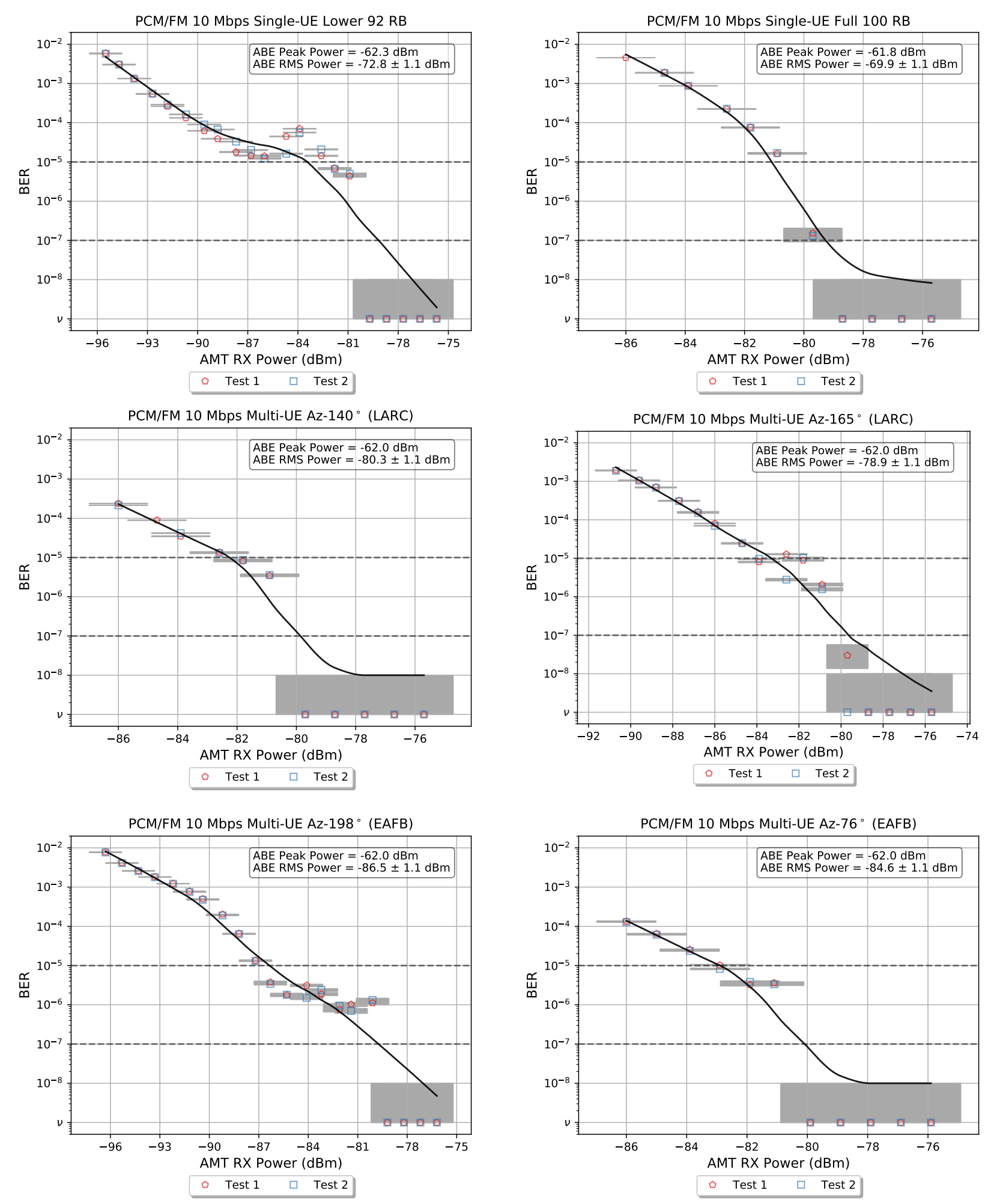

Figure B.6: Main Experiment configurations of PCM/FM 10 Mbps with single-UE and multi-UE LTE ABE. 

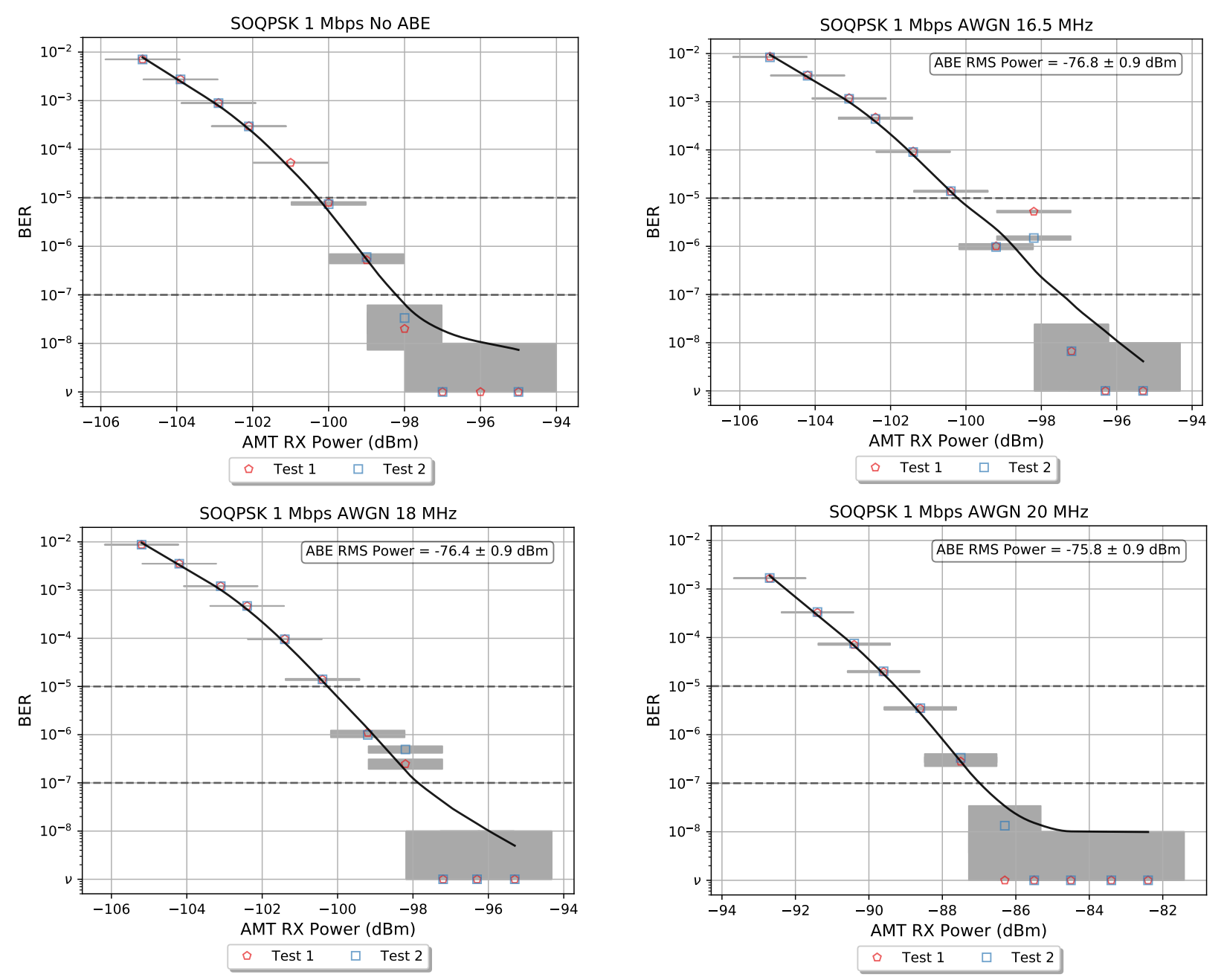

Figure B.7: Main Experiment configurations of SOQPSK 1 Mbps with No ABE and AWGN ABE. 

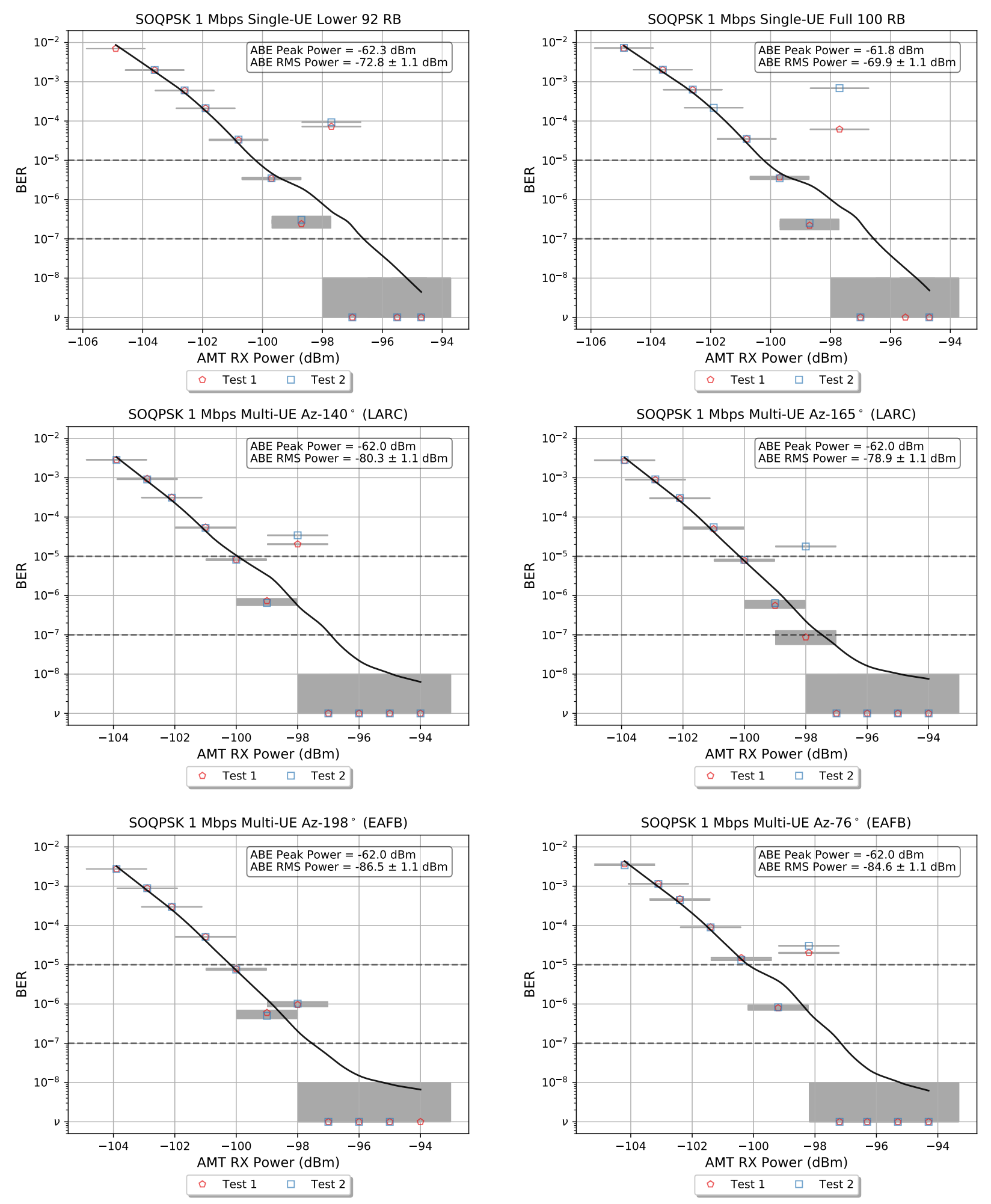

Figure B.8: Main Experiment configurations of SOQPSK 1 Mbps with single-UE and multi-UE LTE ABE. 

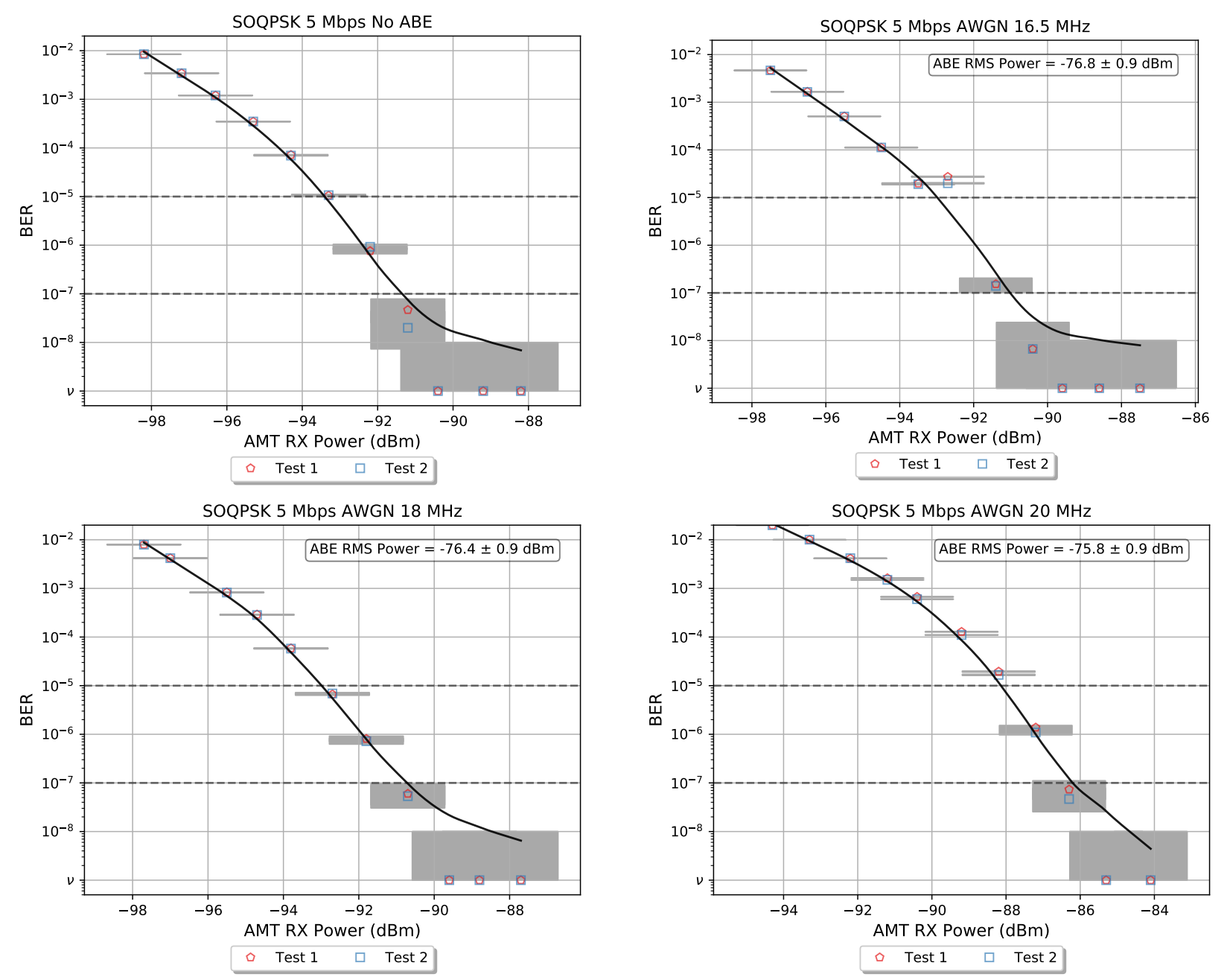

Figure B.9: Main Experiment configurations of SOQPSK 5 Mbps with No ABE and AWGN ABE. 

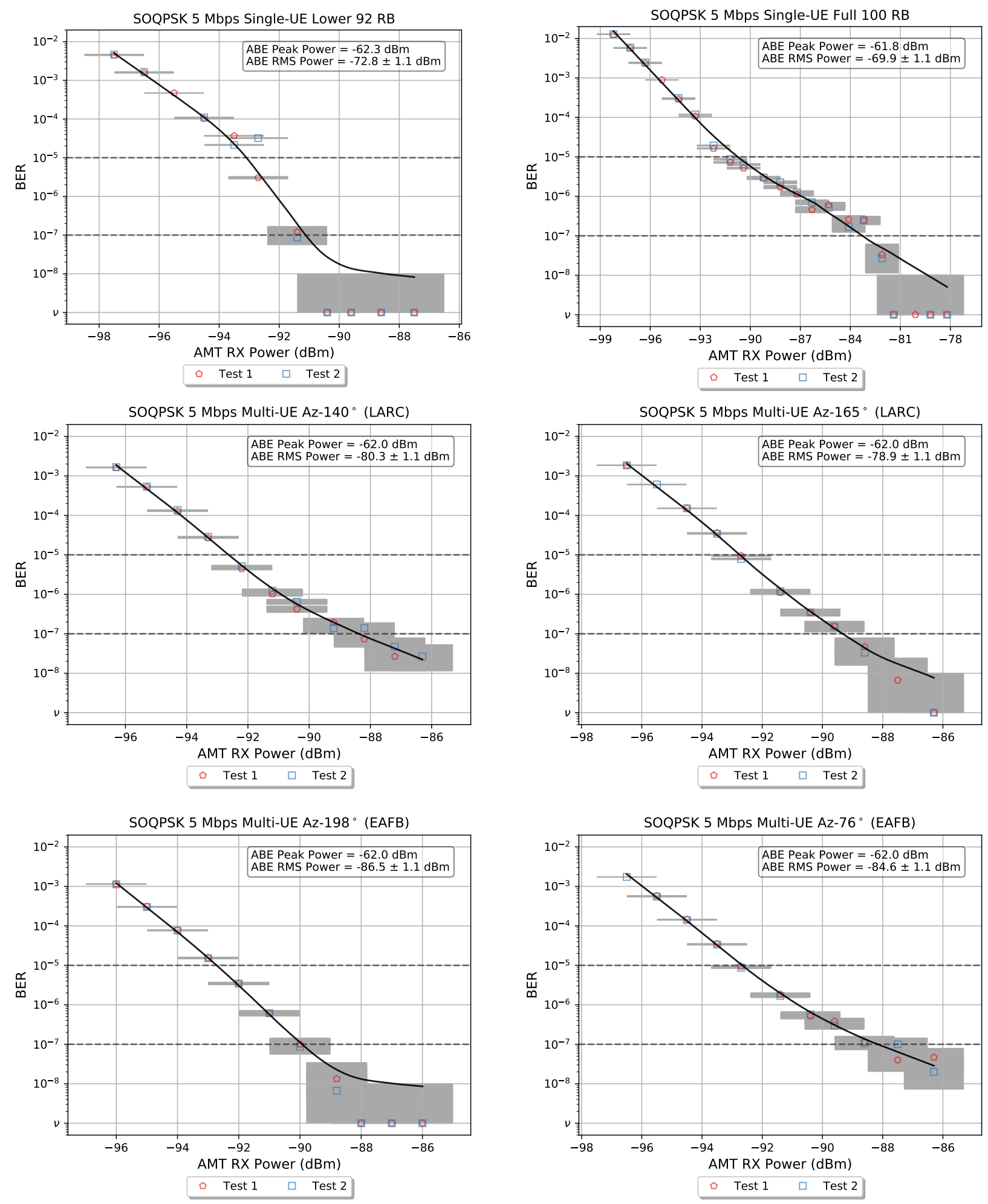

Figure B.10: Main Experiment configurations of SOQPSK 5 Mbps with single-UE and multi-UE LTE ABE. 

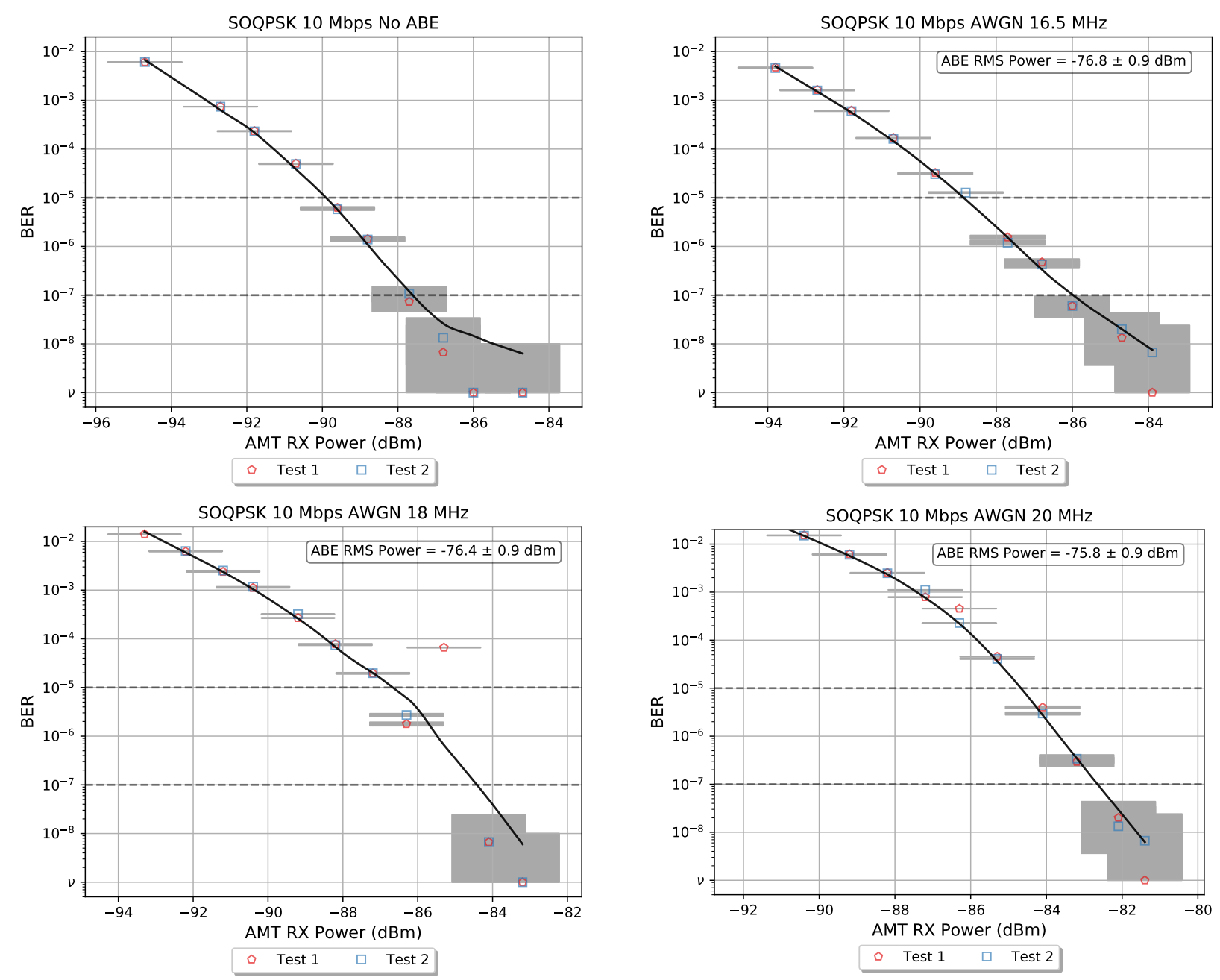

Figure B.11: Main Experiment configurations of SOQPSK 10 Mbps with No ABE and AWGN ABE. 

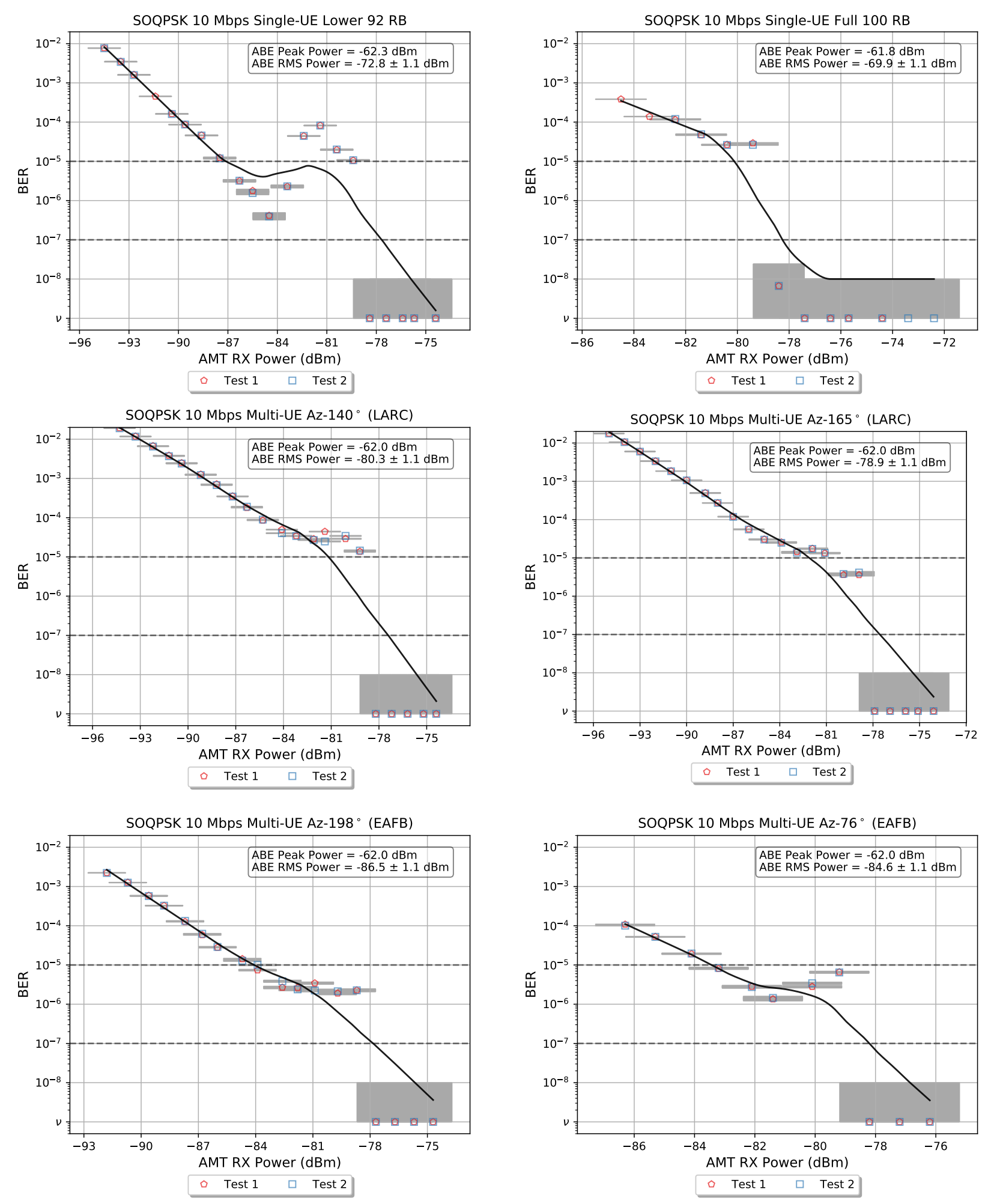

Figure B.12: Main Experiment configurations of SOQPSK 10 Mbps with single-UE and multi-UE LTE ABE. 

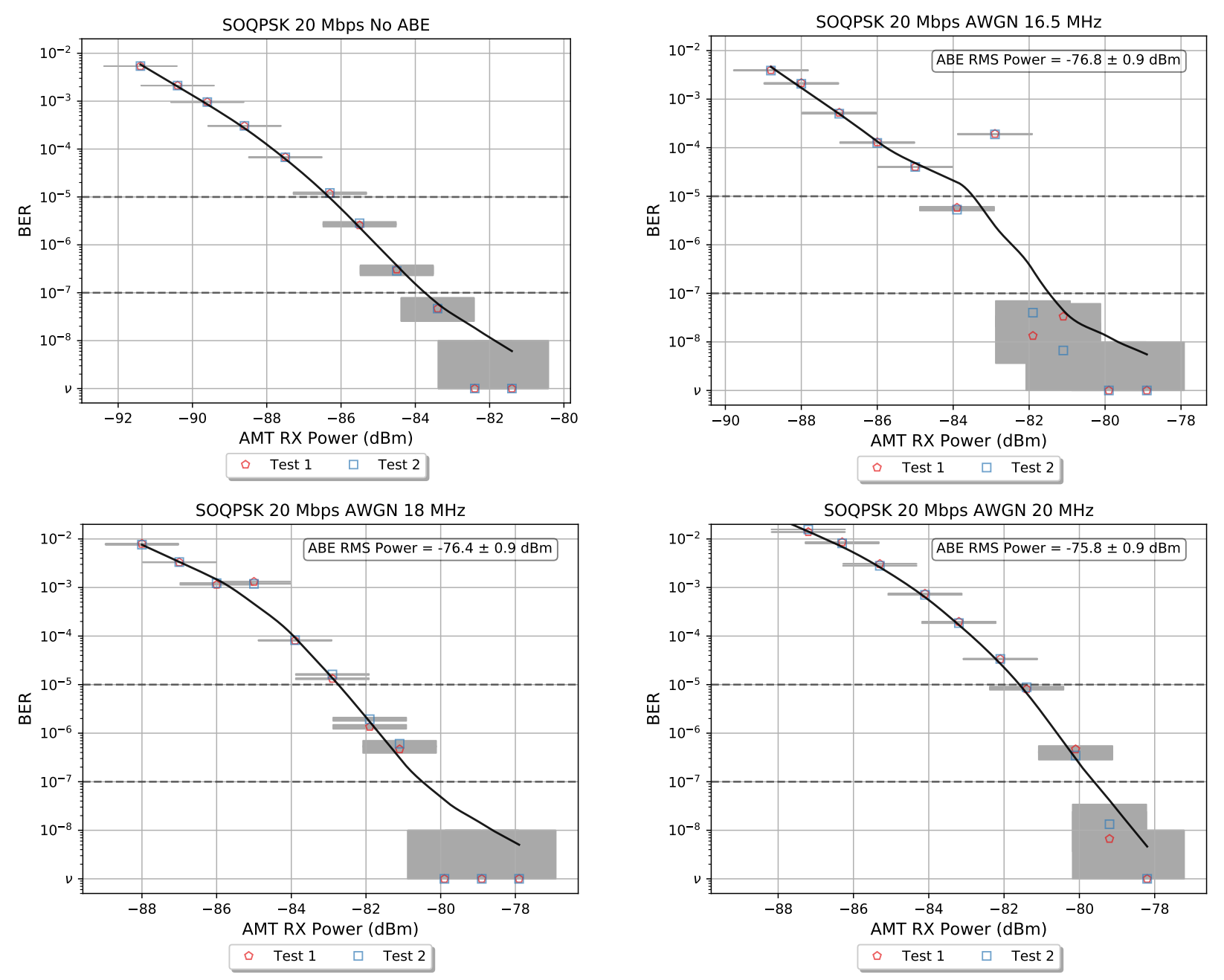

Figure B.13: Main Experiment configurations of SOQPSK 20 Mbps with No ABE and AWGN ABE. 

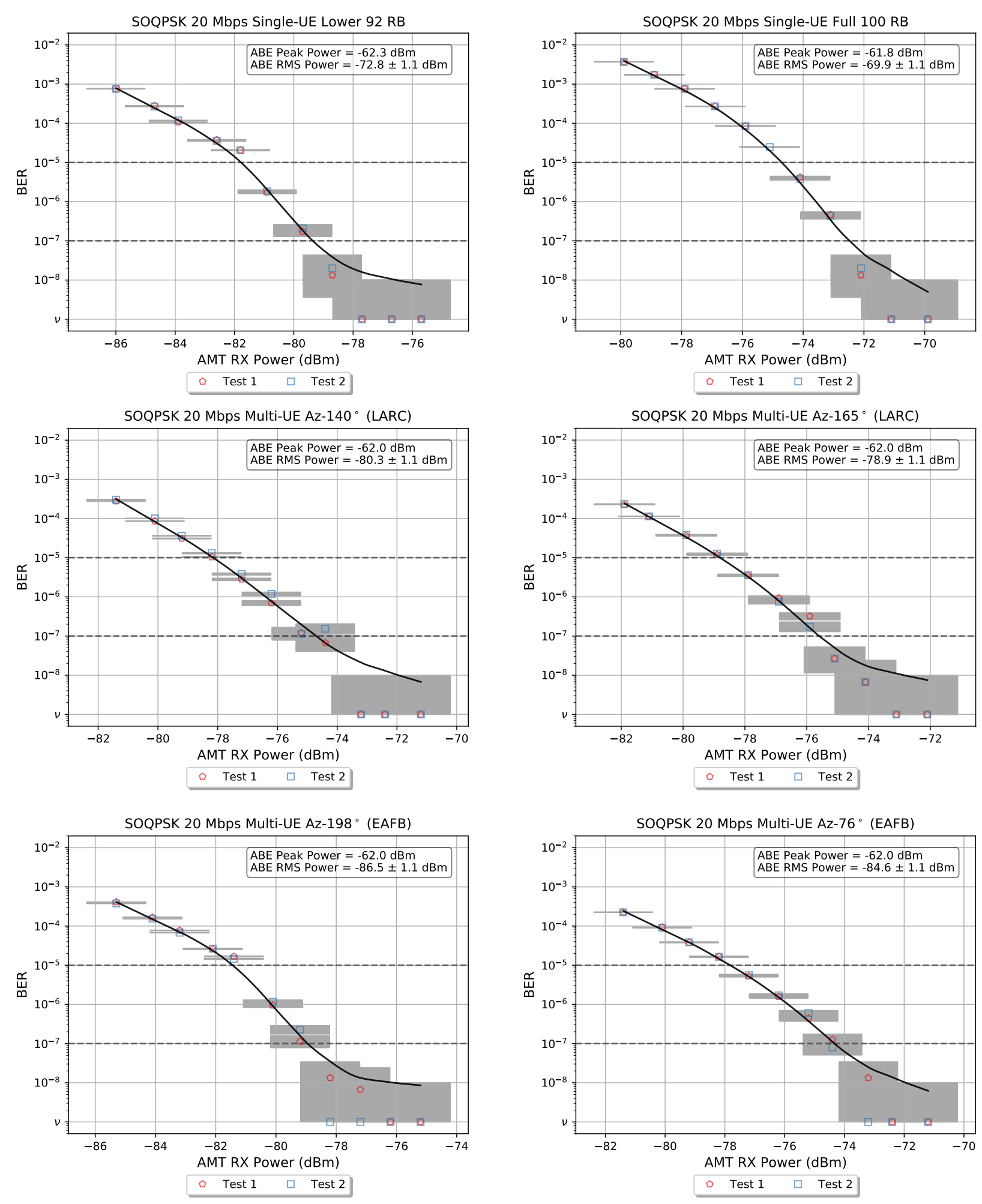

Figure B.14: Main Experiment configurations of SOQPSK 20 Mbps with single-UE and multi-UE LTE ABE. 

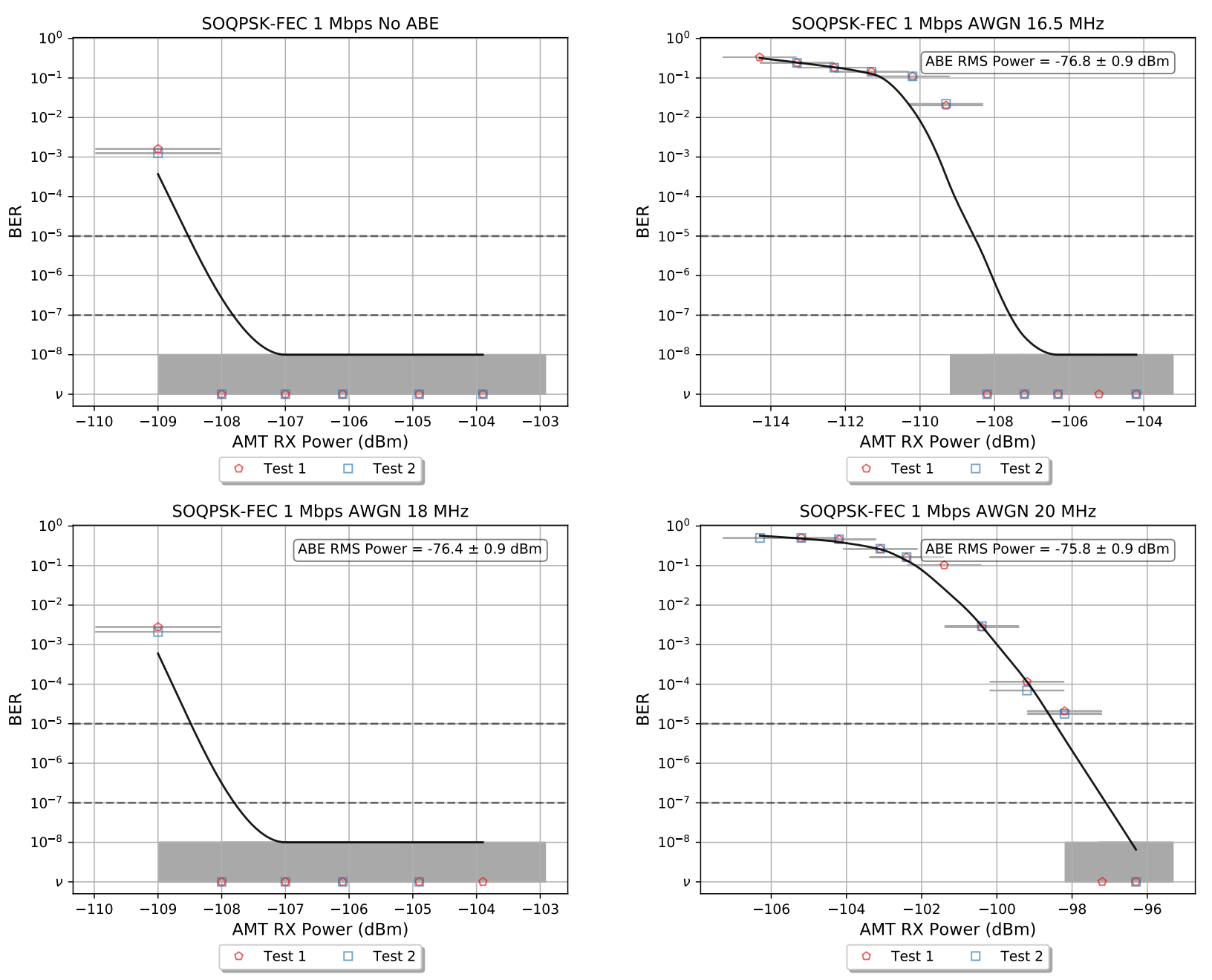

Figure B.15: Main Experiment configurations of SOQPSK-forward error correction (SOQPSK-FEC) 1 Mbps with No $\mathrm{ABE}$ and $\mathrm{AWGN} \mathrm{ABE}$. 

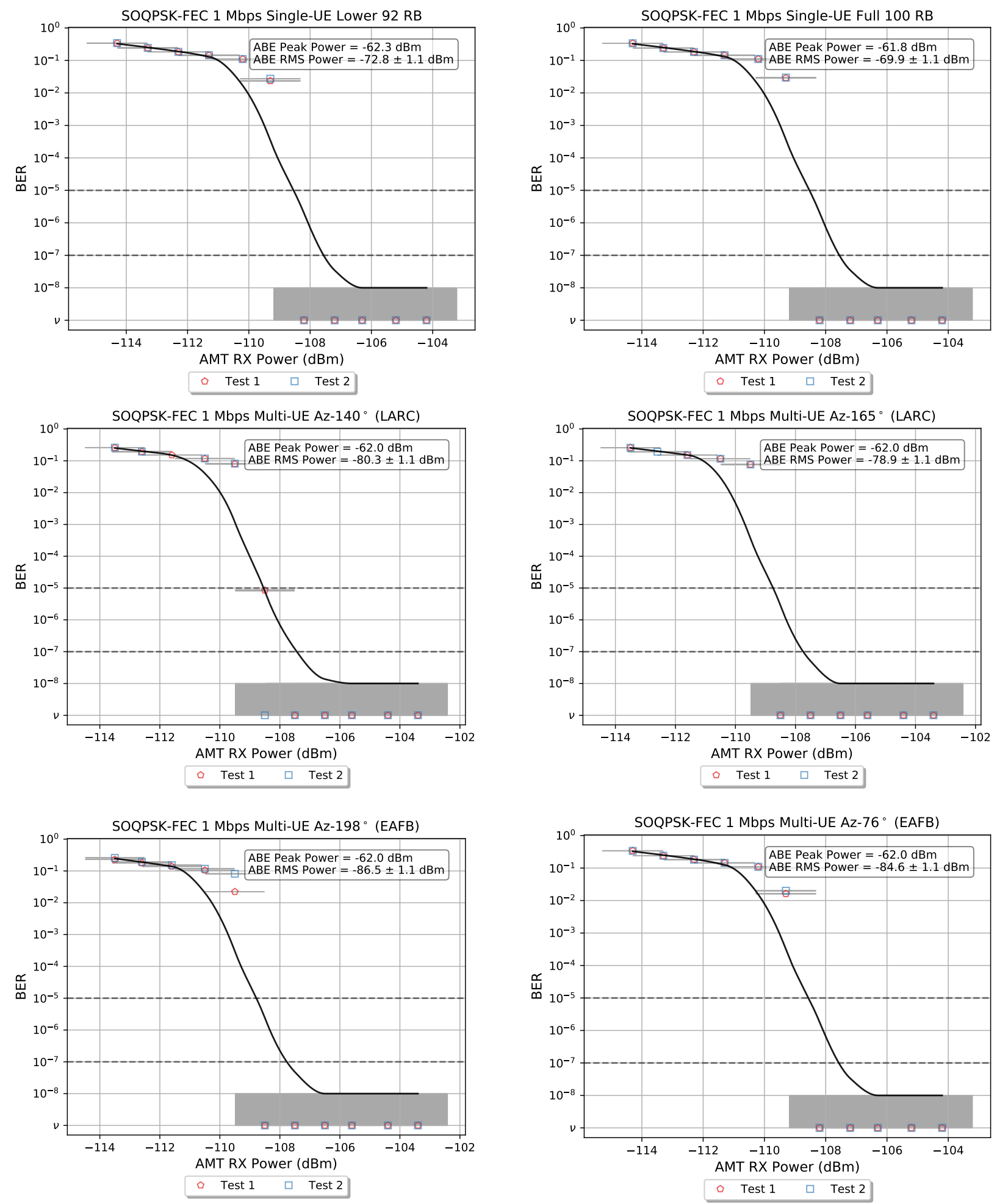

Figure B.16: Main Experiment configurations of SOQPSK-FEC 1 Mbps with single-UE and multi-UE LTE ABE. 

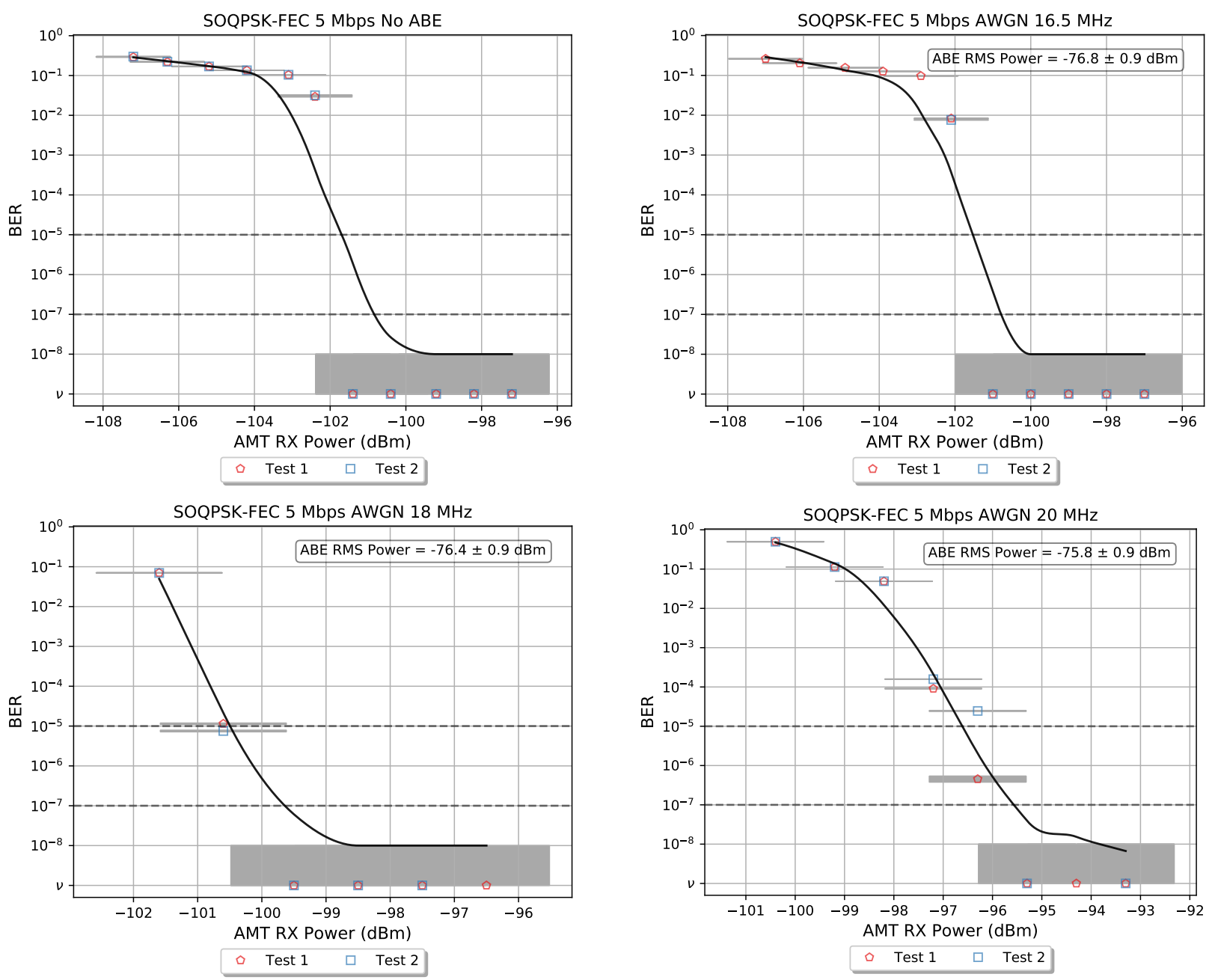

Figure B.17: Main Experiment configurations of SOQPSK-FEC 5 Mbps with No ABE and AWGN ABE. 

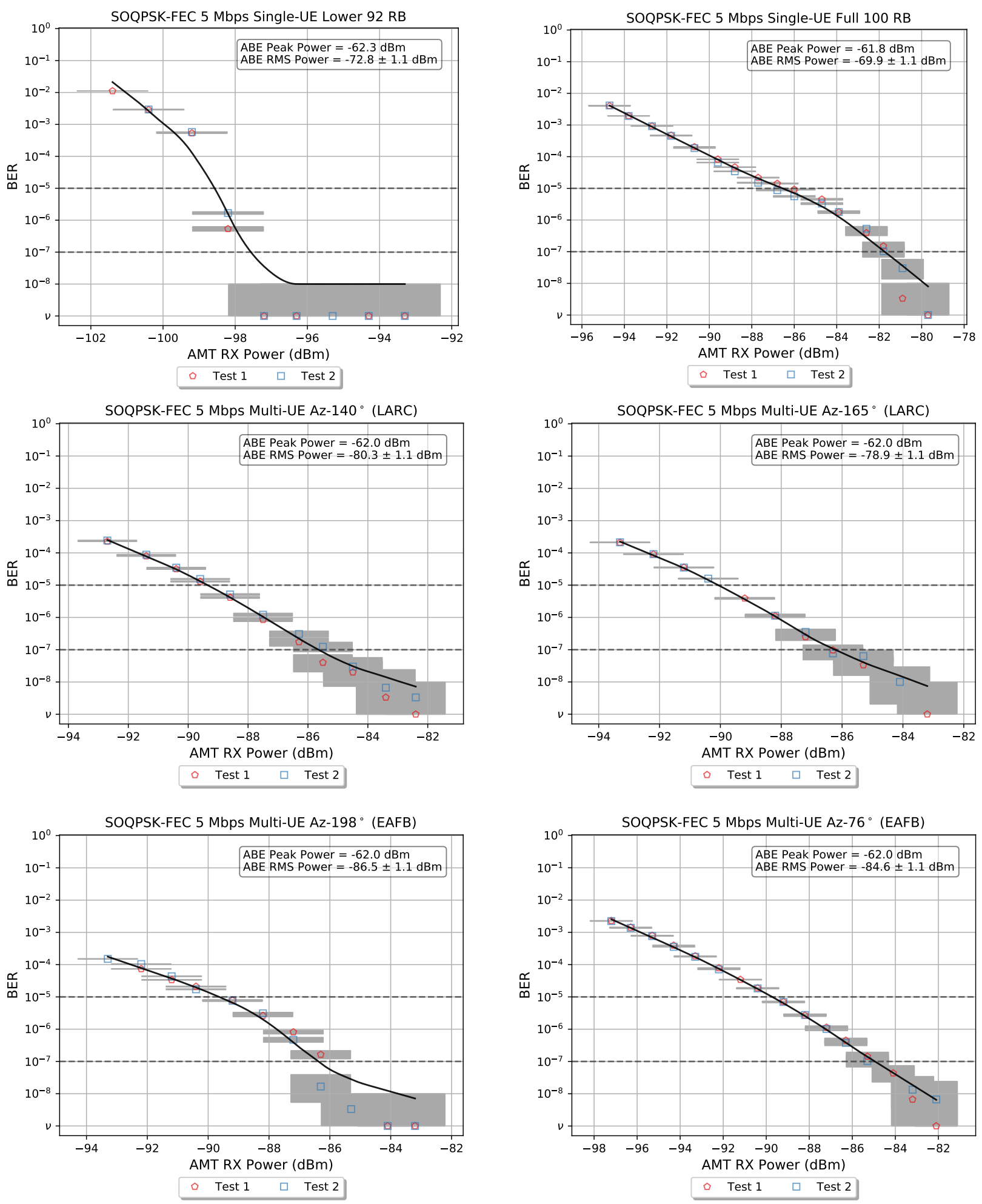

Figure B.18: Main Experiment configurations of SOQPSK-FEC 5 Mbps with single-UE and multi-UE LTE ABE. 

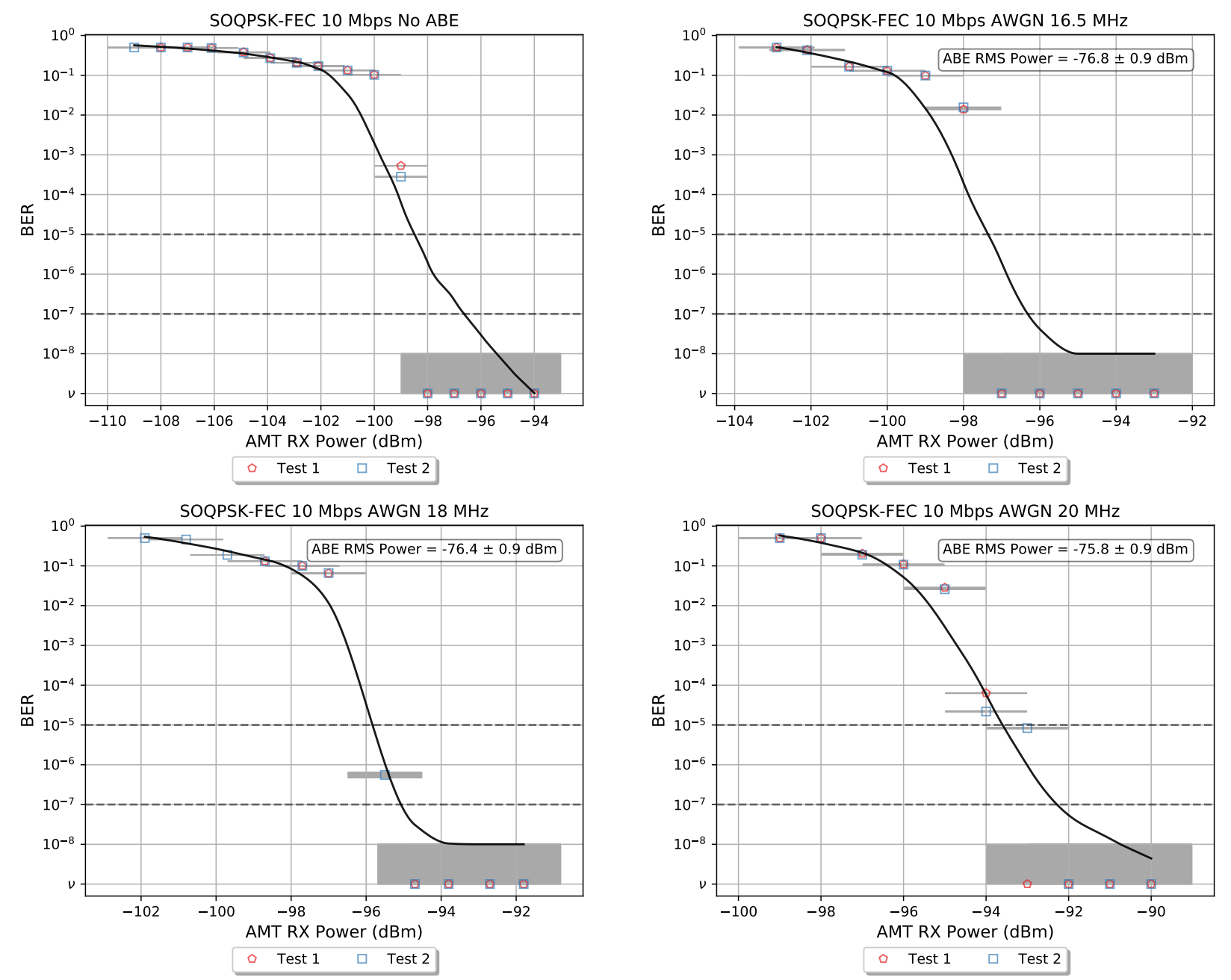

Figure B.19: Main Experiment configurations of SOQPSK-FEC 10 Mbps with No ABE and AWGN ABE. 

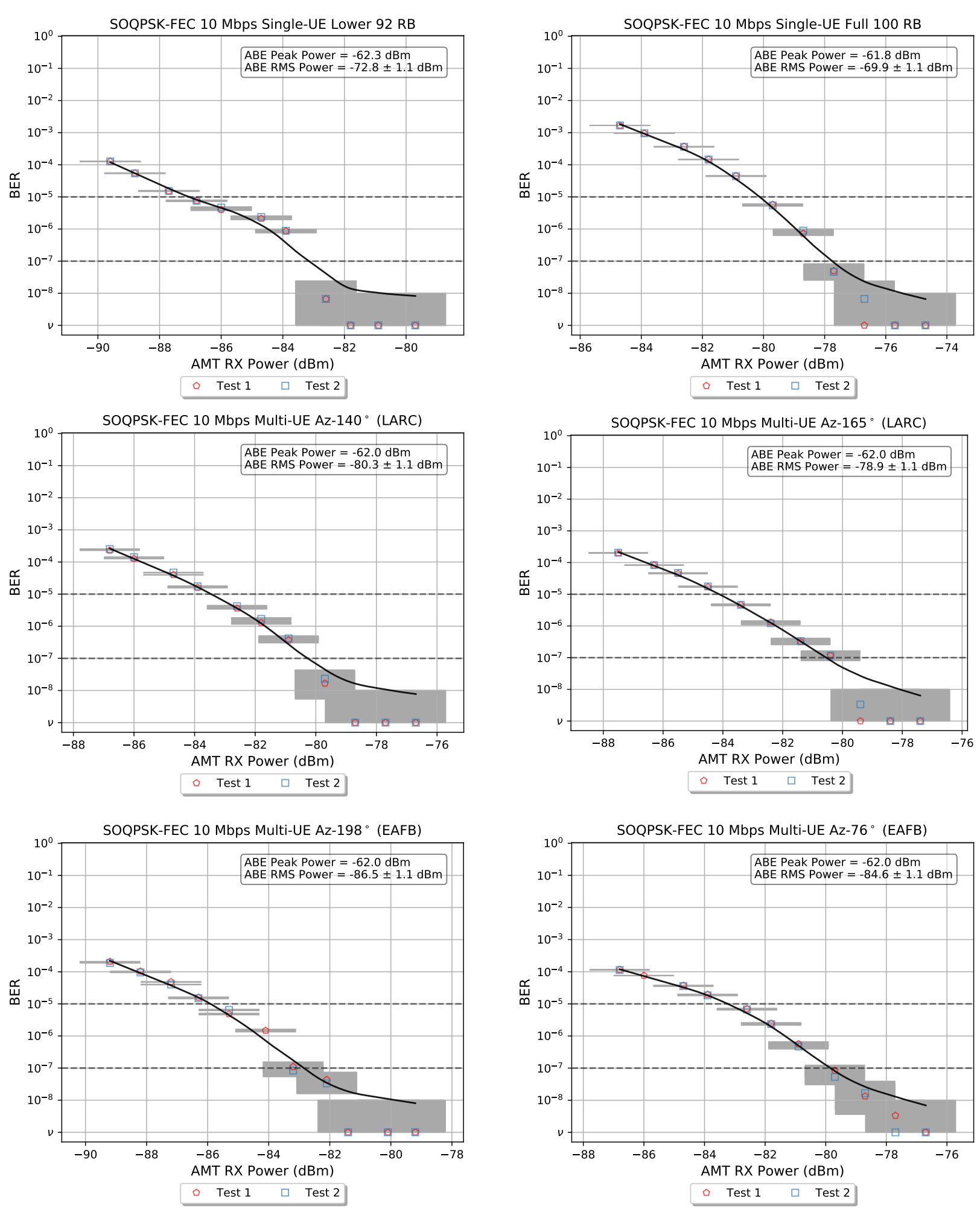

Figure B.20: Main Experiment configurations of SOQPSK-FEC 10 Mbps with single-UE and multi-UE LTE ABE. 

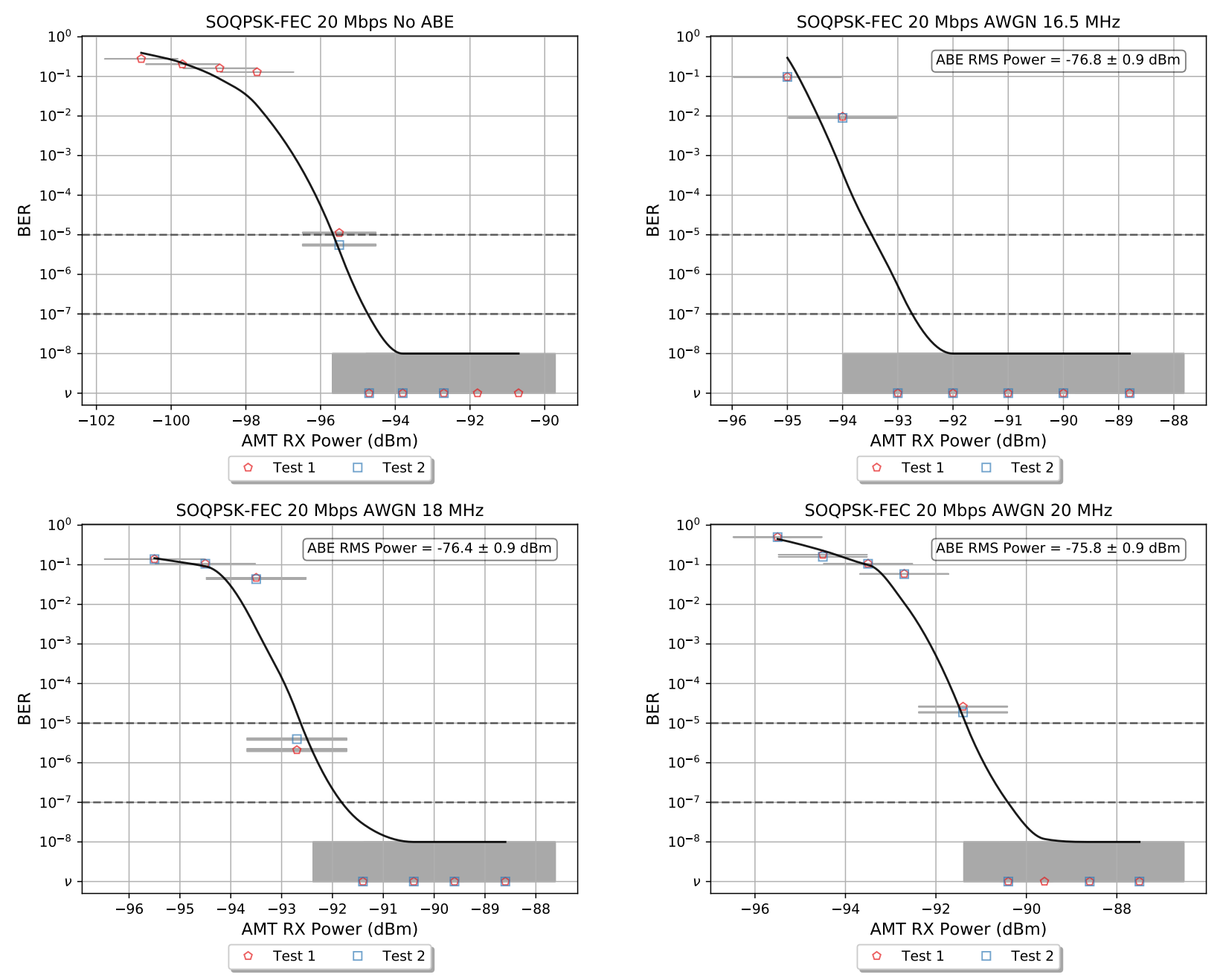

Figure B.21: Main Experiment configurations of SOQPSK-FEC 20 Mbps with No ABE and AWGN ABE. 

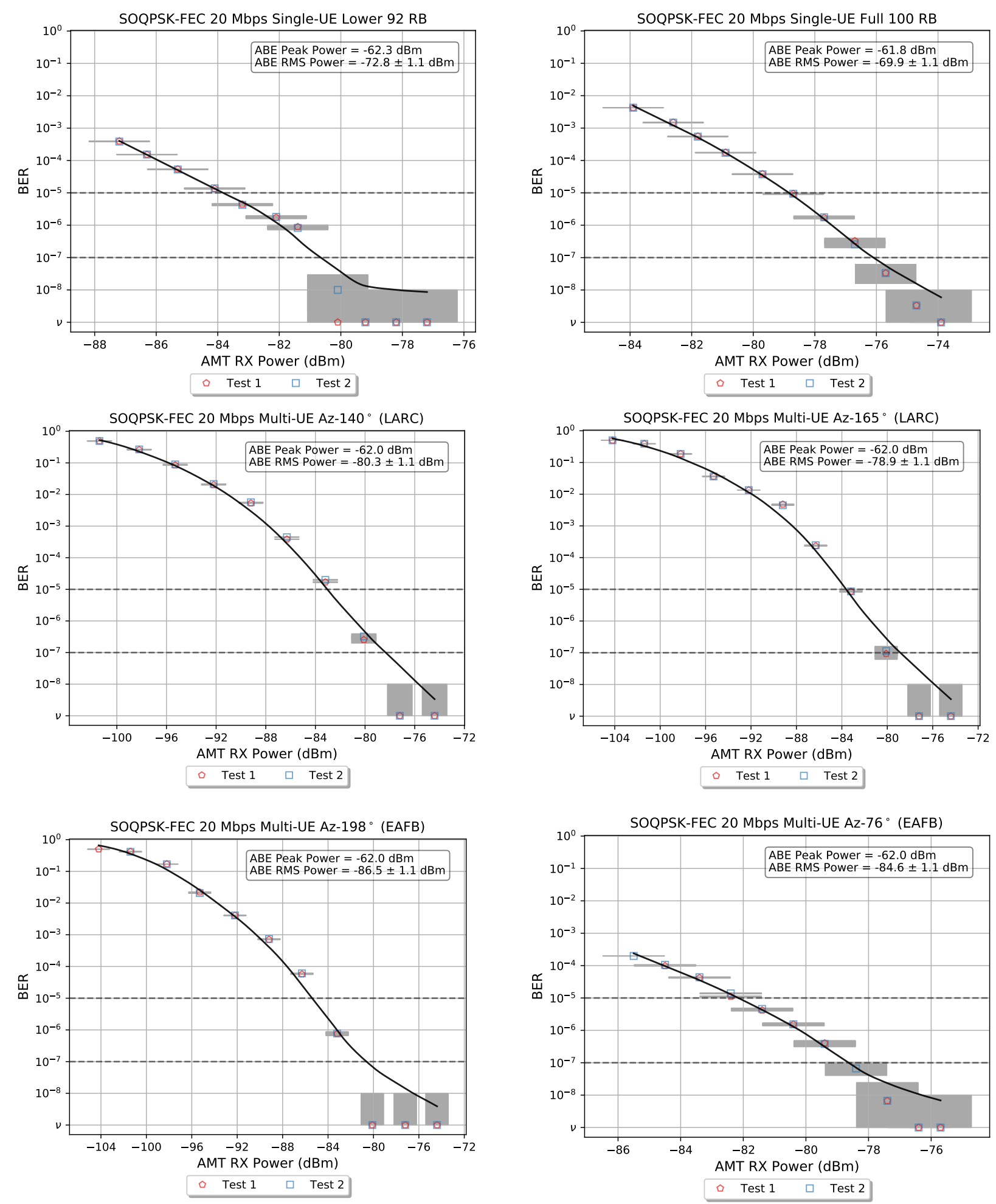

Figure B.22: Main Experiment configurations of SOQPSK-FEC 20 Mbps with single-UE and multi-UE LTE ABE. 

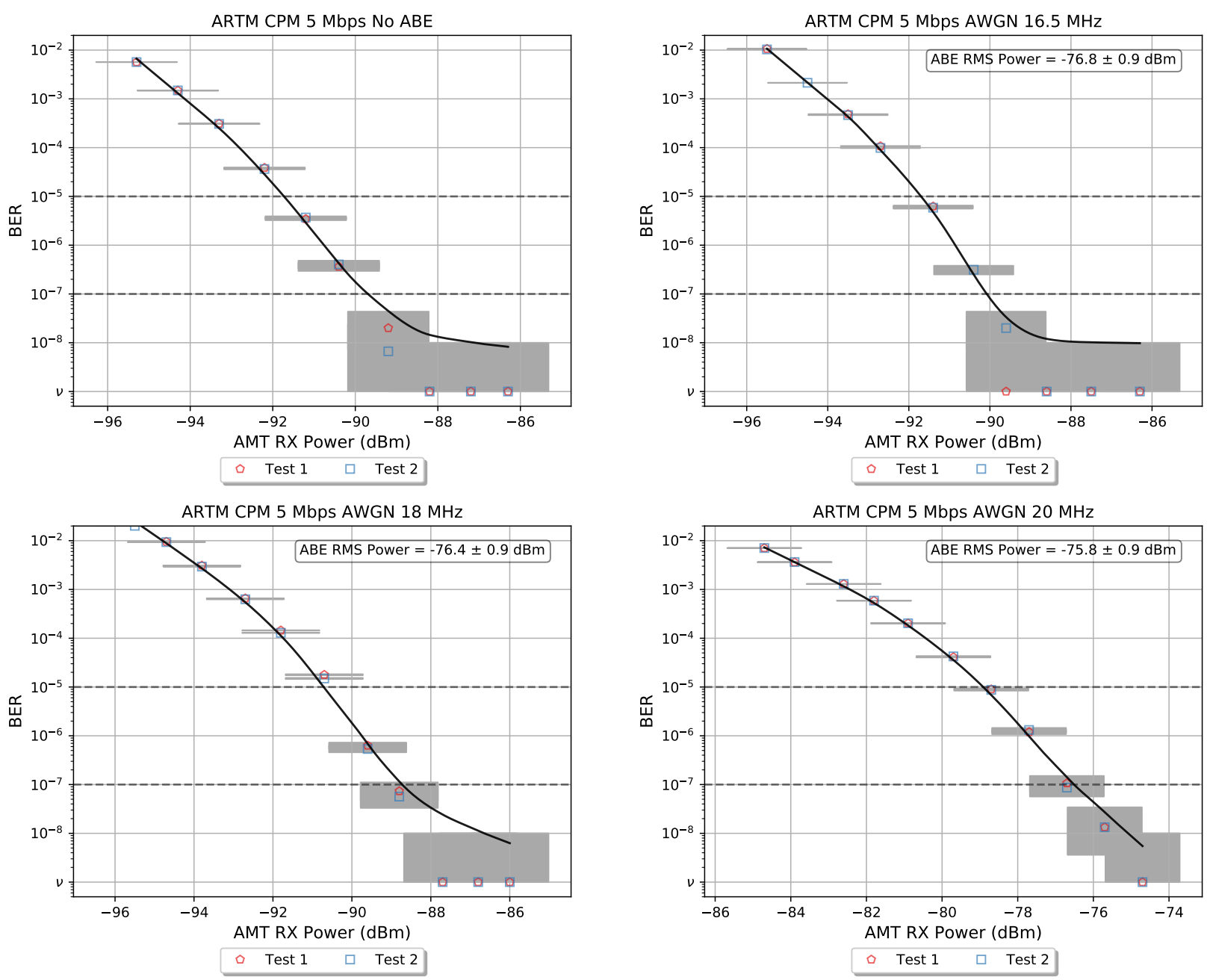

Figure B.23: Main Experiment configurations of ARTM CPM 5 Mbps with No ABE and AWGN ABE. 

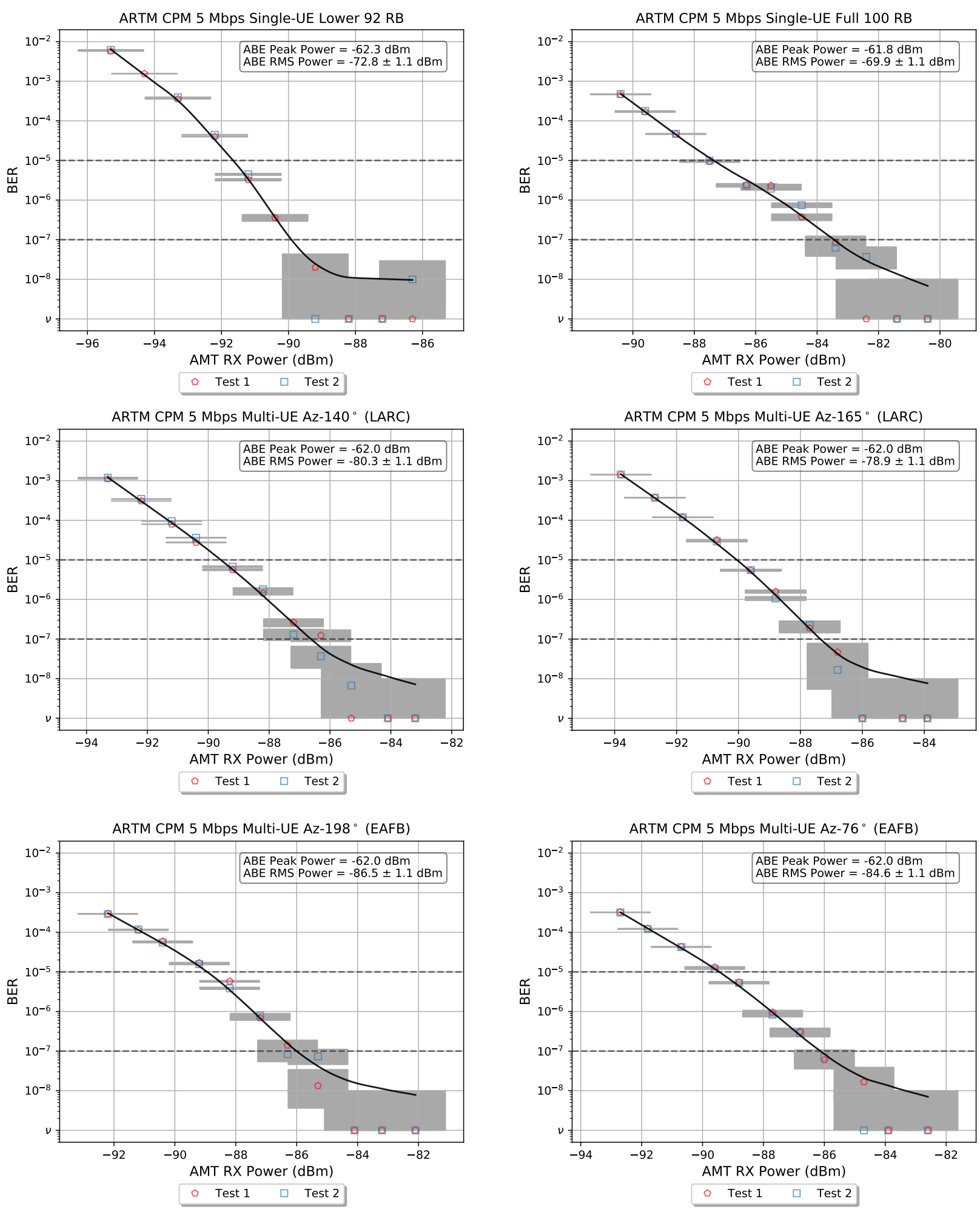

Figure B.24: Main Experiment configurations of ARTM CPM 5 Mbps with single-UE and multi-UE LTE ABE. 

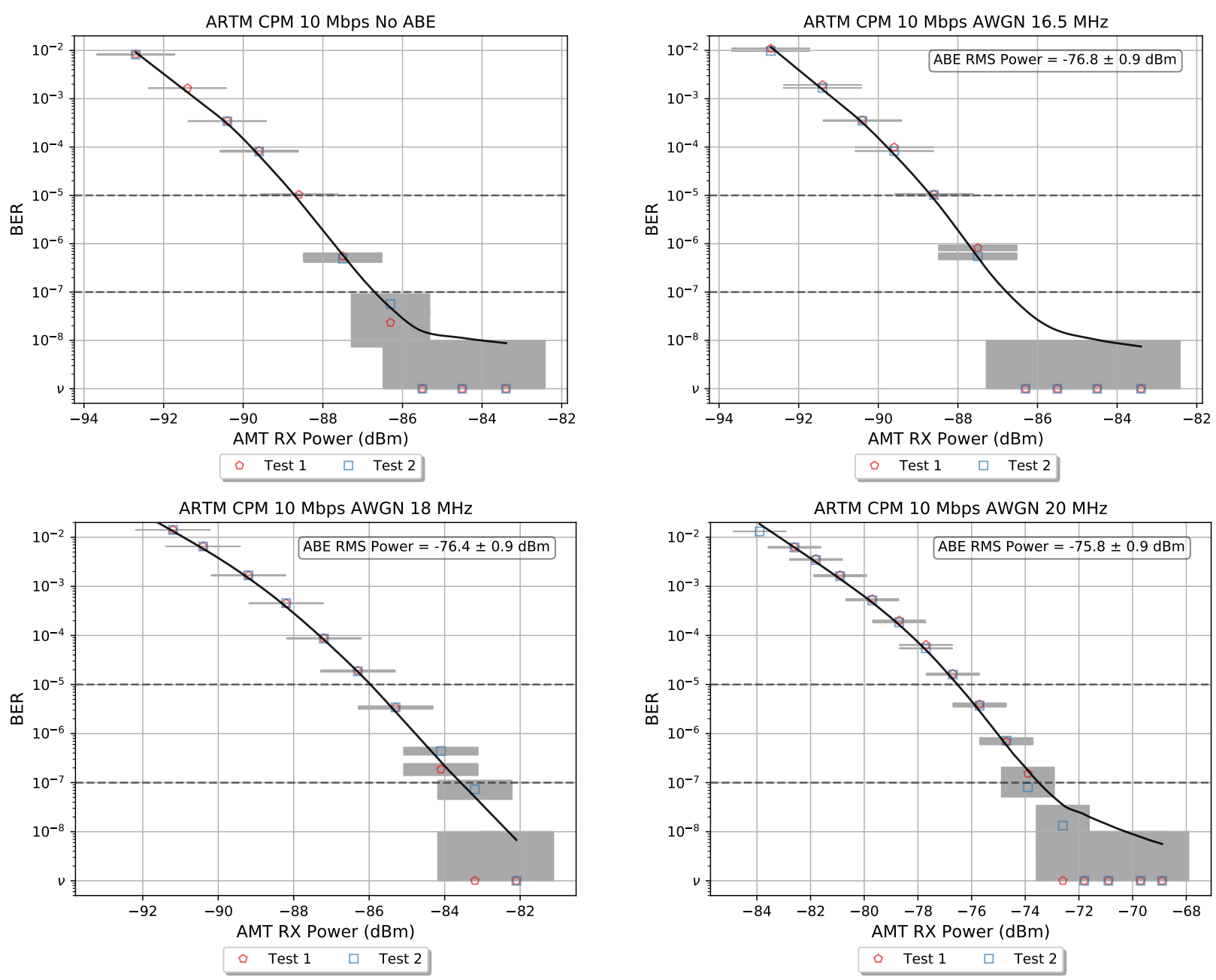

Figure B.25: Main Experiment configurations of ARTM CPM 10 Mbps with No ABE and AWGN ABE. 

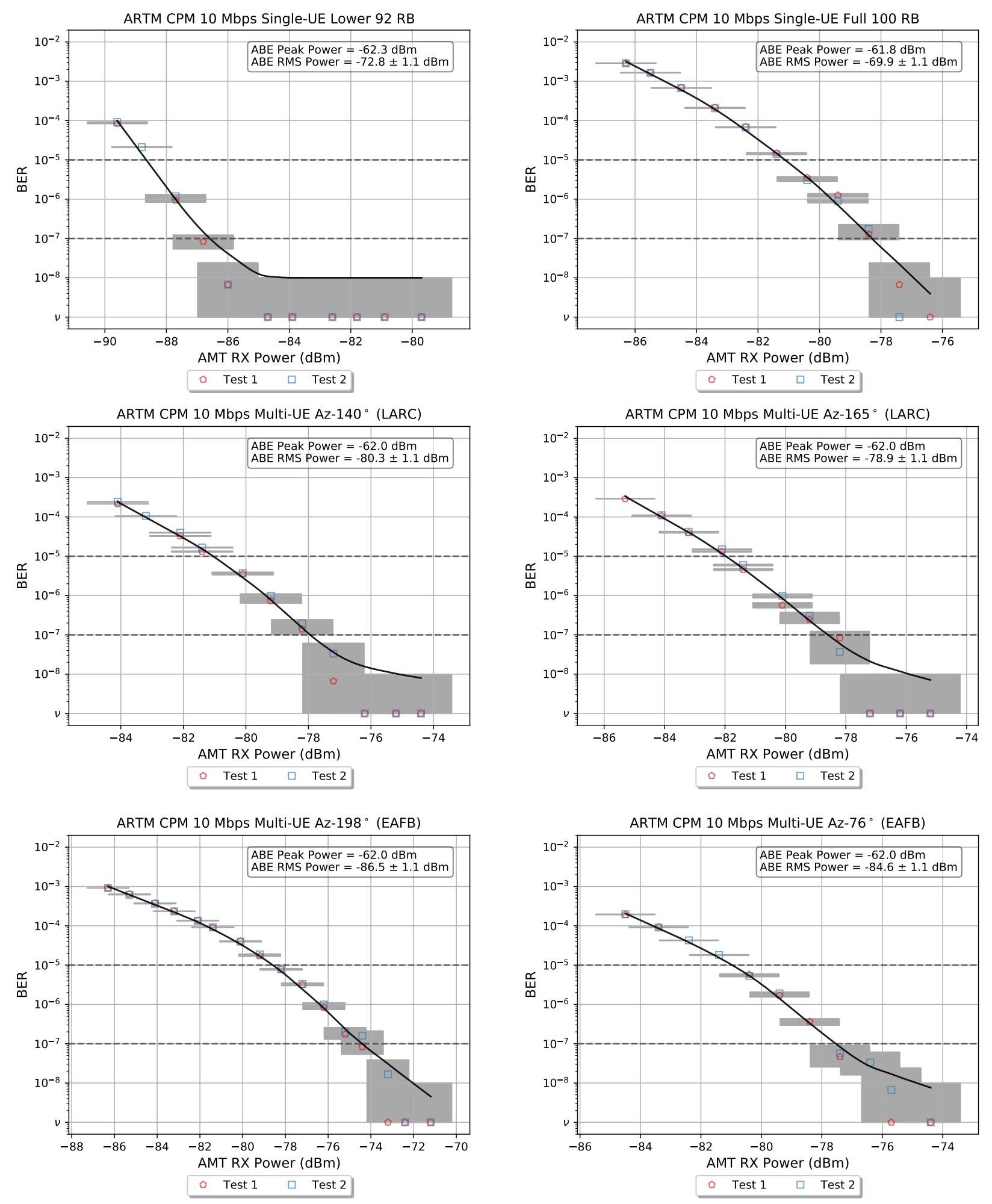

Figure B.26: Main Experiment configurations of ARTM CPM 10 Mbps with single-UE and multi-UE LTE ABE. 

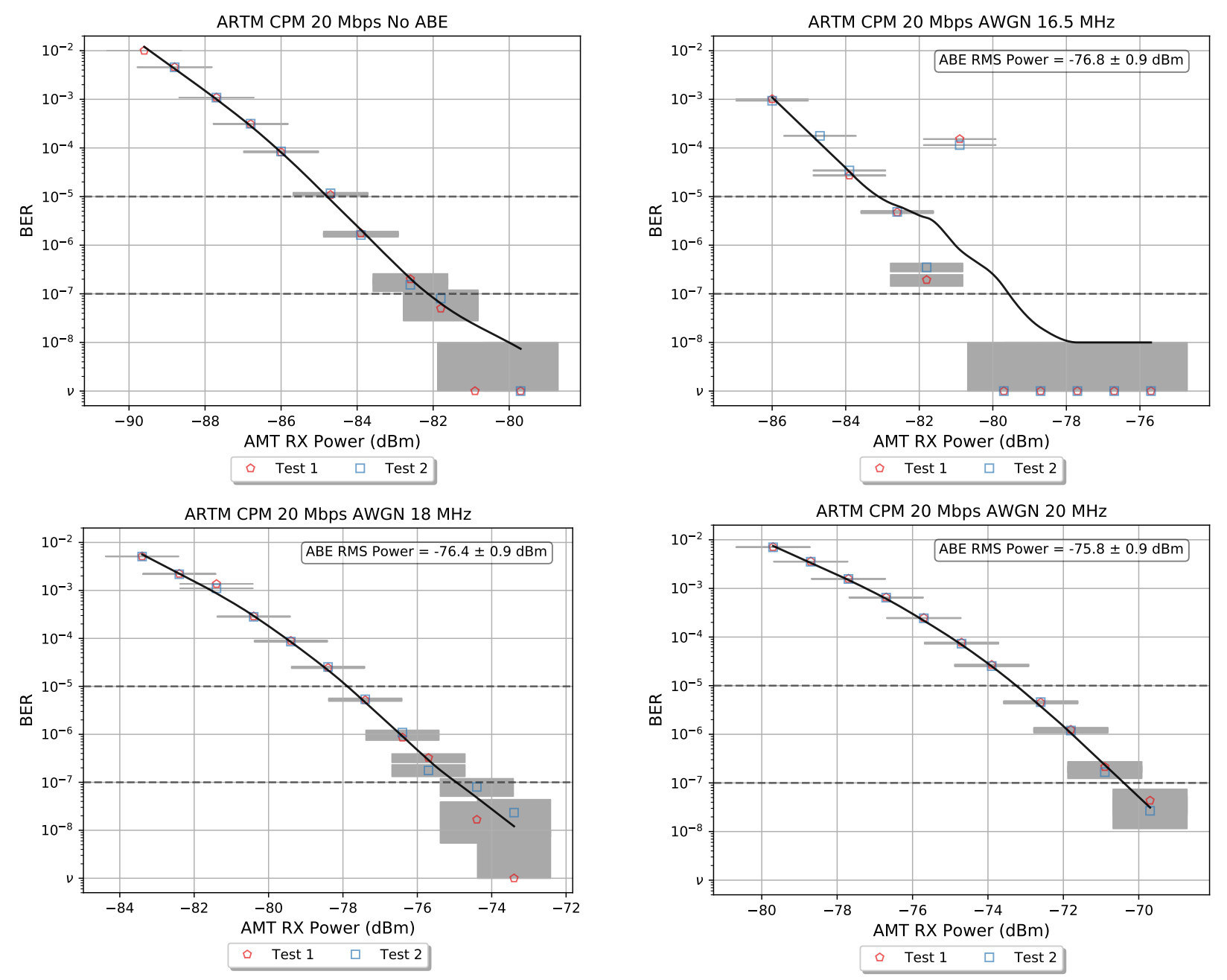

Figure B.27: Main Experiment configurations of ARTM CPM 20 Mbps with No ABE and AWGN ABE. 

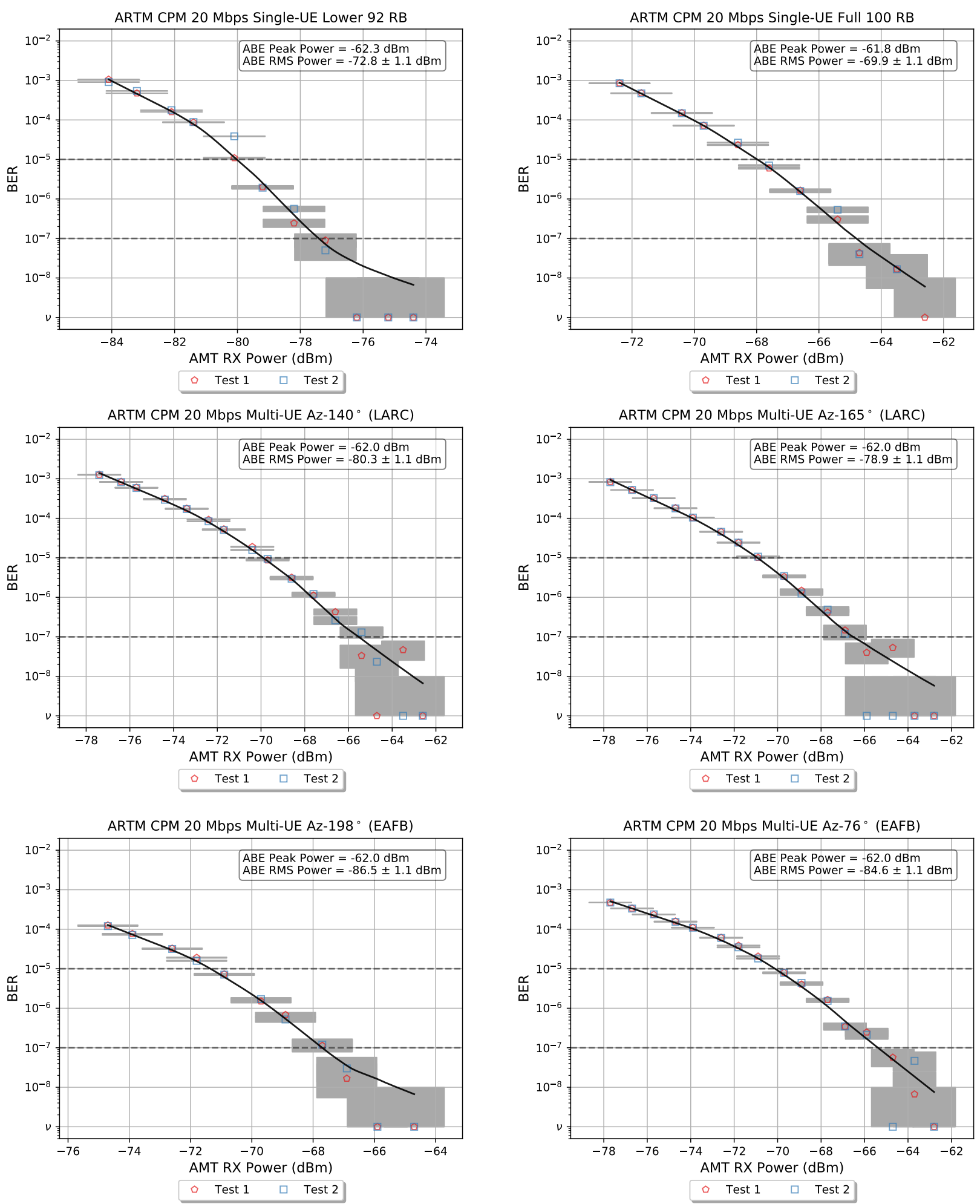

Figure B.28: Main Experiment configurations of ARTM CPM 20 Mbps with single-UE and multi-UE LTE ABE. 


\section{Appendix C}

\section{Results for Side Experiment C: Excess Noise Study}

This appendix presents results for side experiment $\mathrm{C}$, the excess noise study. Only results for the five priority configurations are given here; see Table 2.9.

PCM/FM 1 Mbps

$N_{a}$ Effective $(\mathrm{dBm} / \mathrm{Hz})$
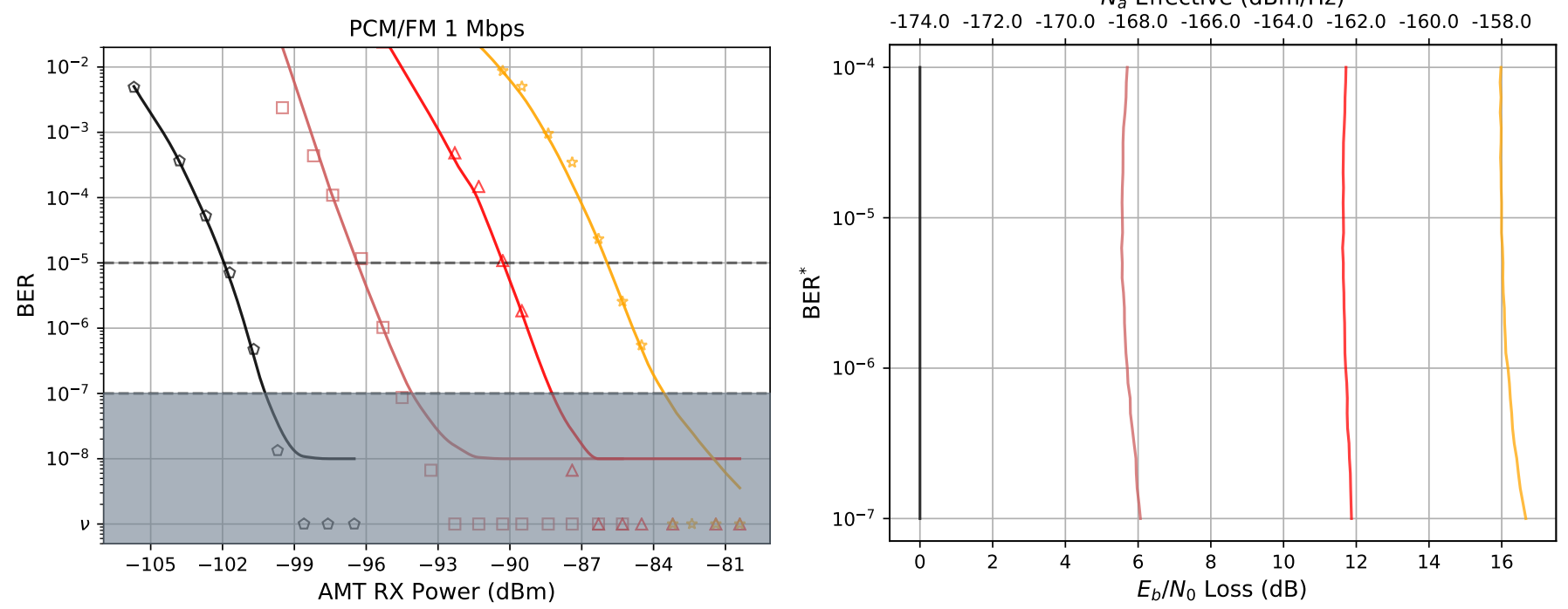

- No Excess Noise

$-N_{R x}=-159.5 \mathrm{dBm} / \mathrm{Hz}$

$N_{R x}=-166.2 \mathrm{dBm} / \mathrm{Hz}$

$-N_{R x}=-154.9 \mathrm{dBm} / \mathrm{Hz}$

Figure C.1: BER vs AMT RX power and BER* vs $\mathrm{N}_{a}$ Effective for all Noise types: PCM/FM 1 Mbps. The region BER $<10^{-7}$ is shaded in the left plot to indicate high relative uncertainty. 
PCM/FM 5 Mbps
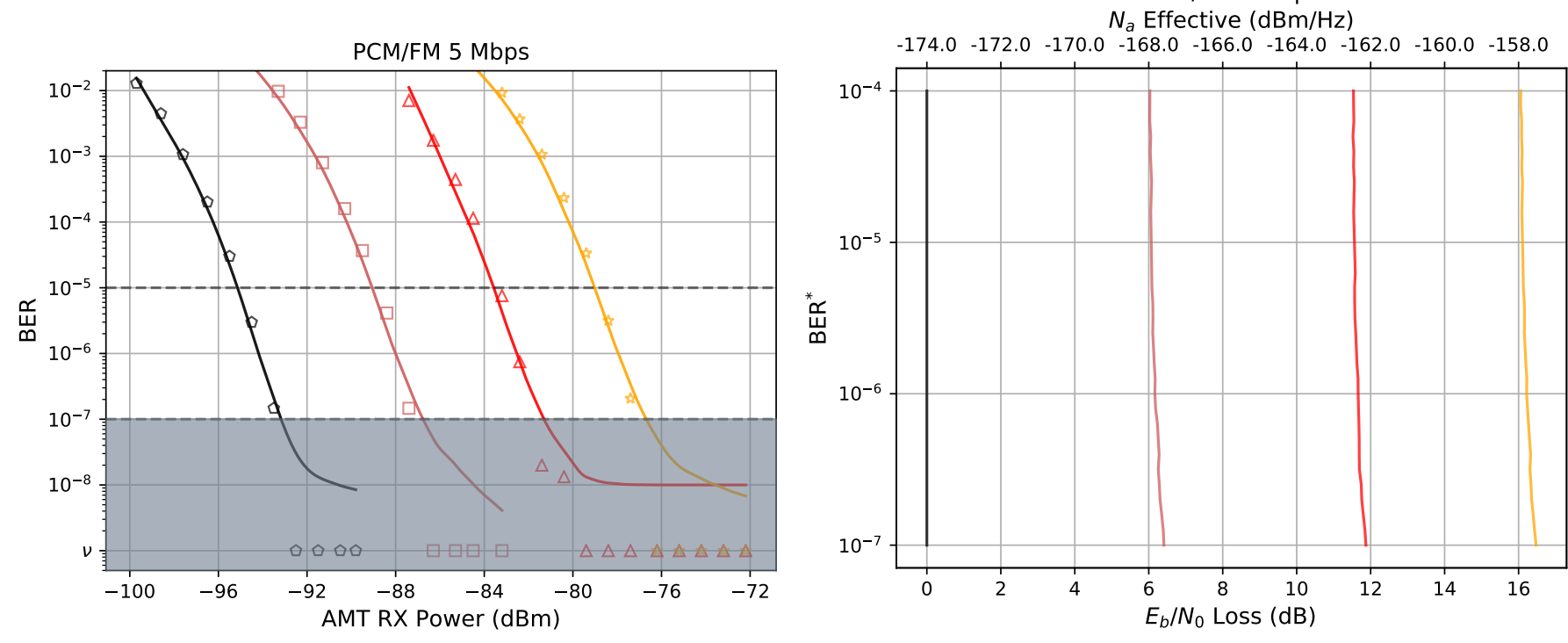

$\begin{array}{ll}- \text { No Excess Noise } & N_{R x}=-159.5 \mathrm{dBm} / \mathrm{Hz} \\ N_{R x}=-166.2 \mathrm{dBm} / \mathrm{Hz} & \quad N_{R x}=-154.9 \mathrm{dBm} / \mathrm{Hz}\end{array}$

Figure C.2: BER vs AMT RX power and BER* vs $\mathrm{N}_{a}$ Effective for all Noise types: PCM/FM 5 Mbps. The region BER $<10^{-7}$ is shaded in the left plot to indicate high relative uncertainty.

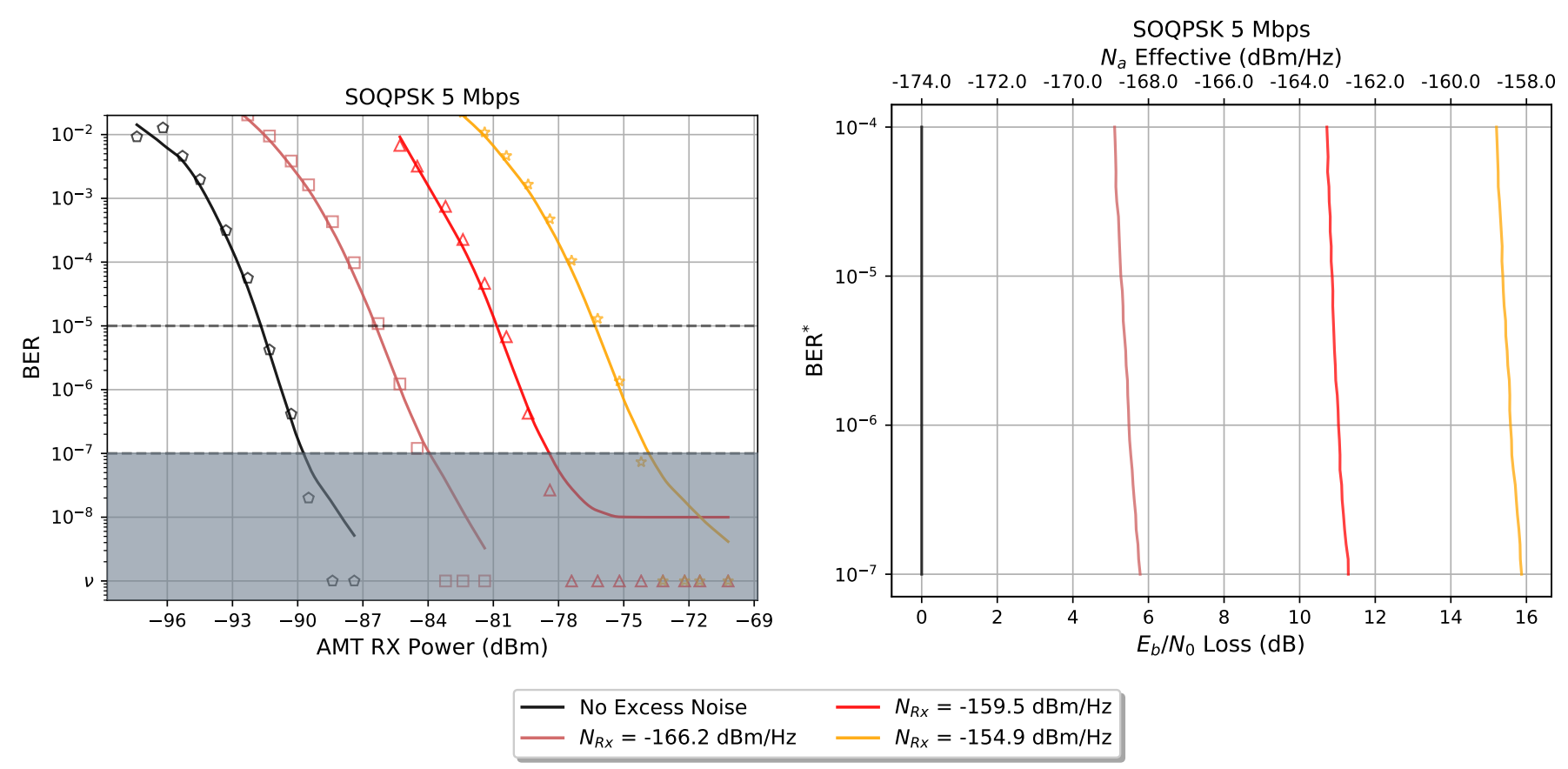

Figure C.3: BER vs AMT RX power and BER* vs $\mathrm{N}_{a}$ Effective for all Noise types: SOQPSK 5 Mbps. The region BER $<10^{-7}$ is shaded in the left plot to indicate high relative uncertainty. 

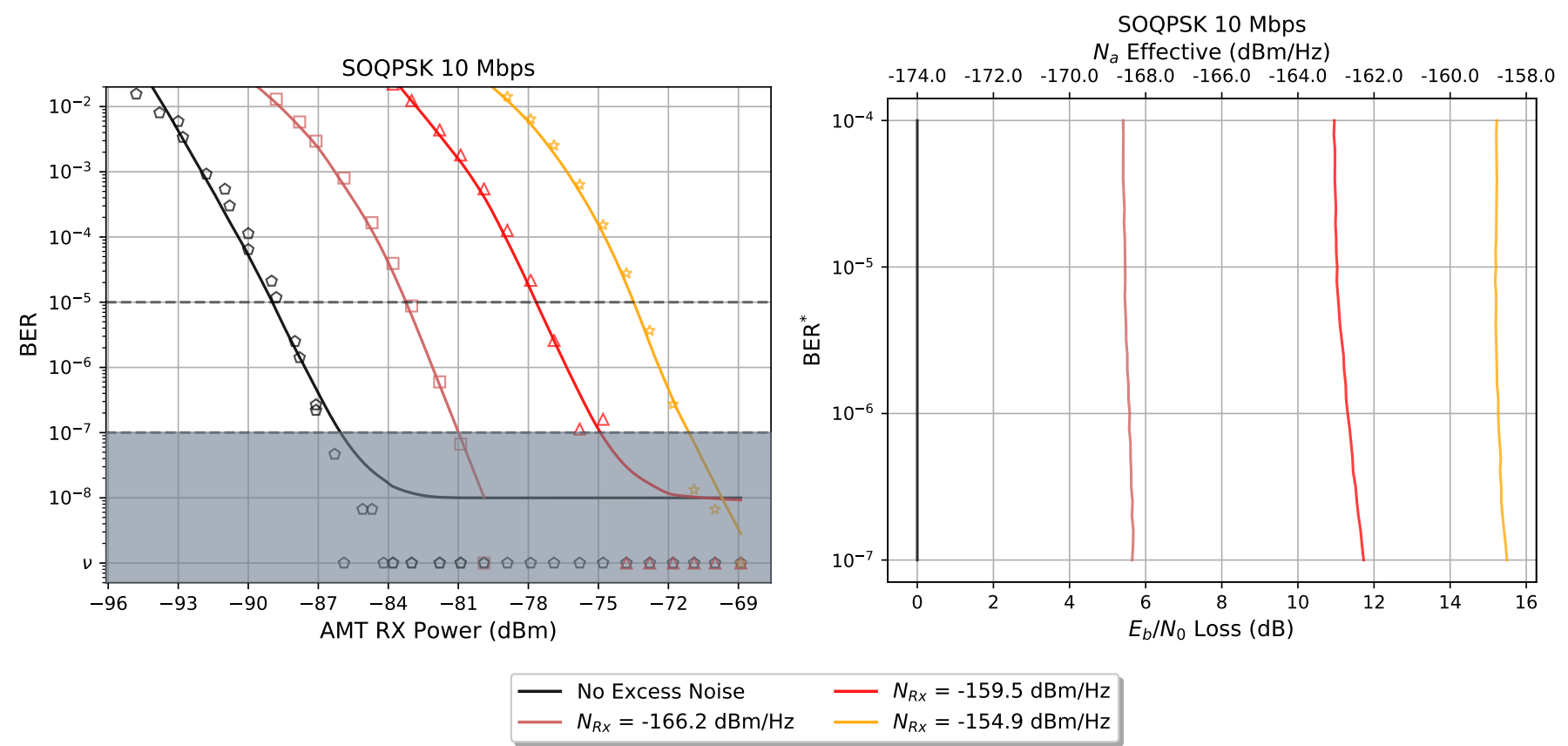

Figure C.4: BER vs AMT RX power and BER* vs $\mathrm{N}_{a}$ Effective for all Noise types: SOQPSK 10 Mbps. The region BER $<10^{-7}$ is shaded in the left plot to indicate high relative uncertainty.

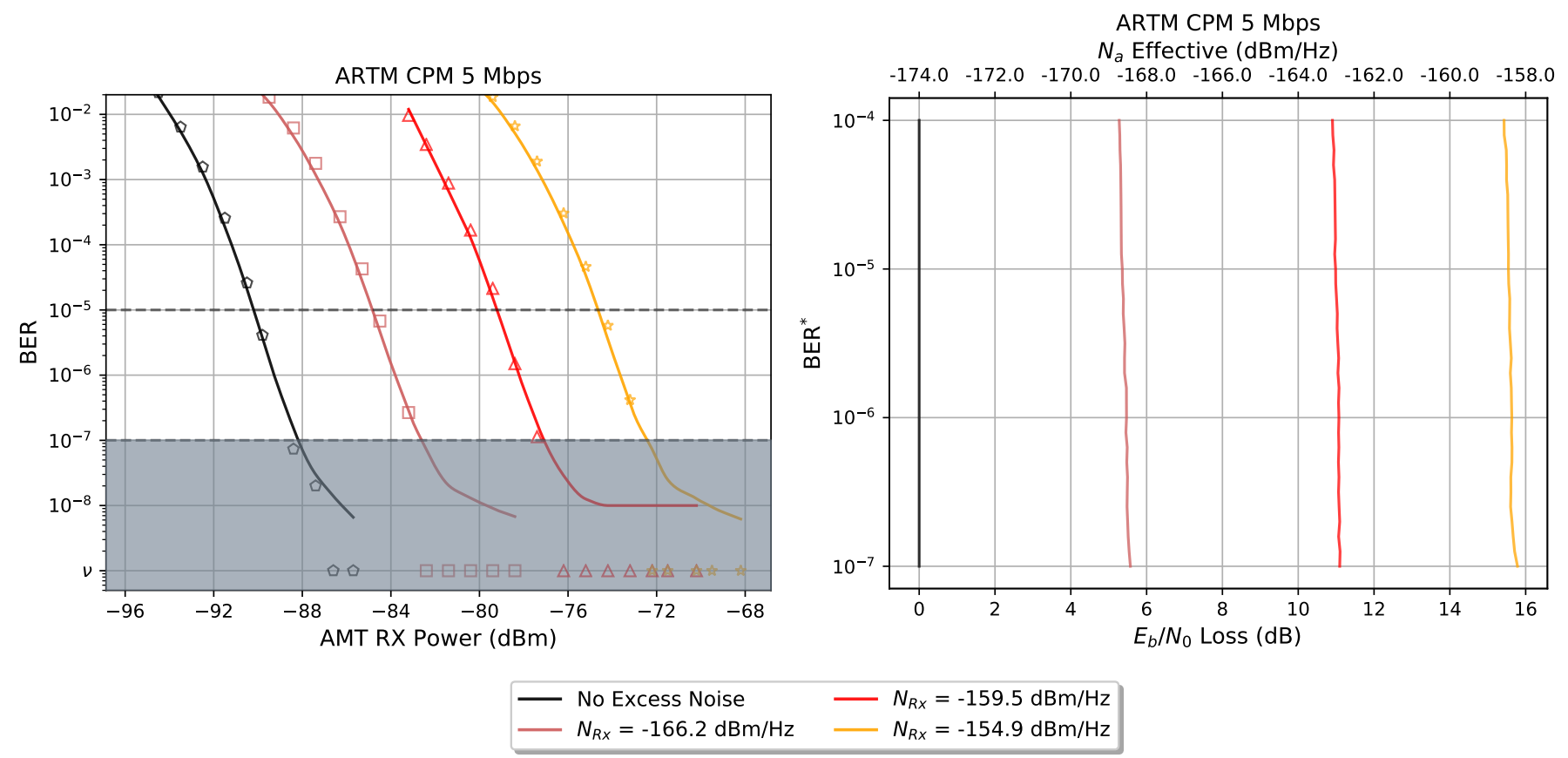

Figure C.5: BER vs AMT RX power and BER* vs $\mathrm{N}_{a}$ Effective for all Noise types: ARTM CPM 5 Mbps. The region BER $<10^{-7}$ is shaded in the left plot to indicate high relative uncertainty. 


\section{Appendix D}

\section{Receiver-Reported Eb/No Scatterplots}

In this appendix, we present scatterplots of receiver-reported $E_{b} / N_{0}$ for the priority main experiment configurations collected in Bedford; see Table 2.4. We include these plots to demonstrate that receiver-reported $E_{b} / N_{0}$ is not a reliable indicator of BER performance in the presence of ABE. Since $E_{b} / N_{0}$ is a ratio of in-band quantities, this observation is expected.

In each figure, the points in the time-series for reported $E_{b} / N_{0}$ are plotted versus AMT signal power. To convey the uncertainty in the AMT signal power at the input to the receiver, the x-axis coordinate is drawn from a normal distribution with mean equal to the calibrated value and standard deviation equal to $0.43 \mathrm{~dB}$, the testbed uncertainty given in Table A.3. The median RX-reported $E_{b} / N_{0}$ for each test condition is denoted with a black circle, and the medians are connected with lines that are the same color as the point clouds for each ABE type.

From these plots, it can be seen that many $\mathrm{ABE}$ types yield RX-reported $E_{b} / N_{0}$ responses that are inconsistent with BER impact relative to the no-ABE case. For example, in Figure D. 3 for SOQPSK 5 Mbps, the AWGN $18 \mathrm{MHz}$ case indicates a large impact, with negative reported $E_{b} / N_{0}$ following a nonlinear response versus AMT power. By contrast, Figure 5.3, which presents the BER response for this condition, shows that AWGN $18 \mathrm{MHz}$ has only a small relative impact that is uniform with AMT power. 


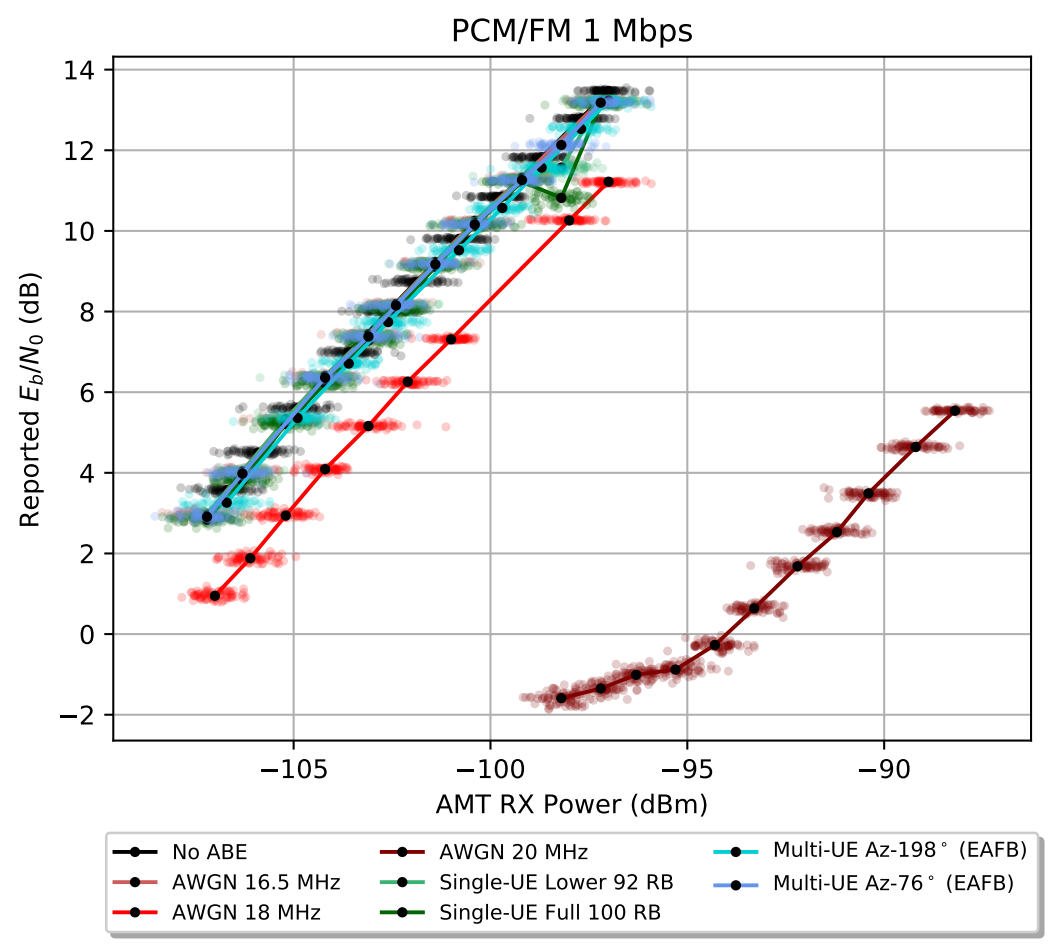

Figure D.1: Reported $E_{b} / N_{0}$ vs AMT RX power for select ABE types: PCM/FM 1 Mbps

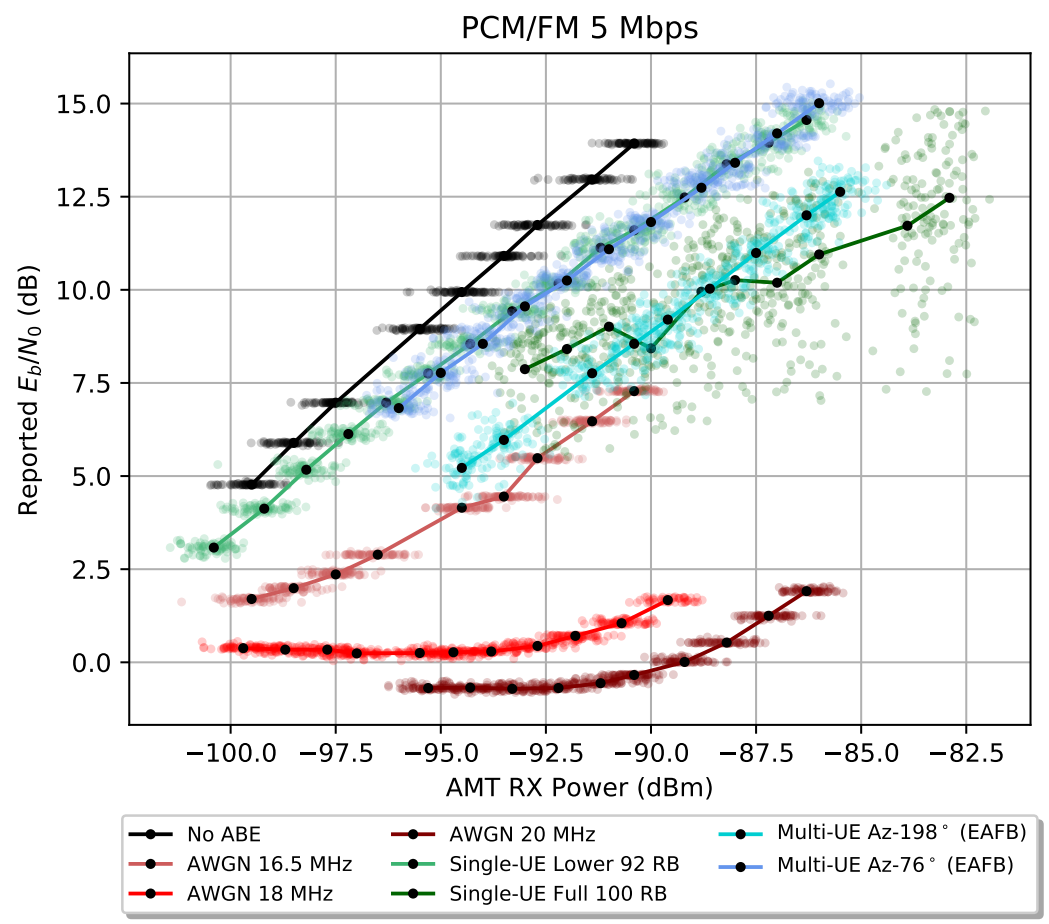

Figure D.2: Reported $E_{b} / N_{0}$ vs AMT RX power for select ABE types: PCM/FM 5 Mbps 


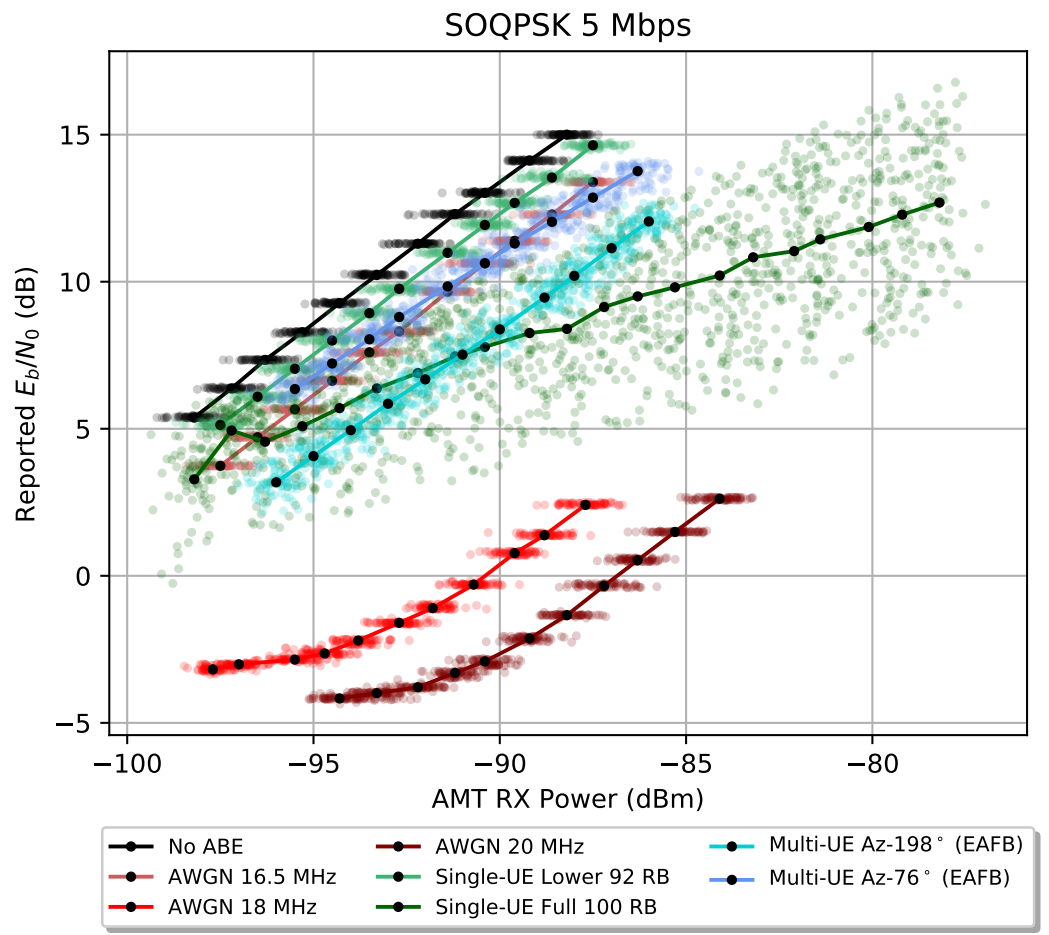

Figure D.3: Reported $E_{b} / N_{0}$ vs AMT RX power for select ABE types: SOQPSK 5 Mbps

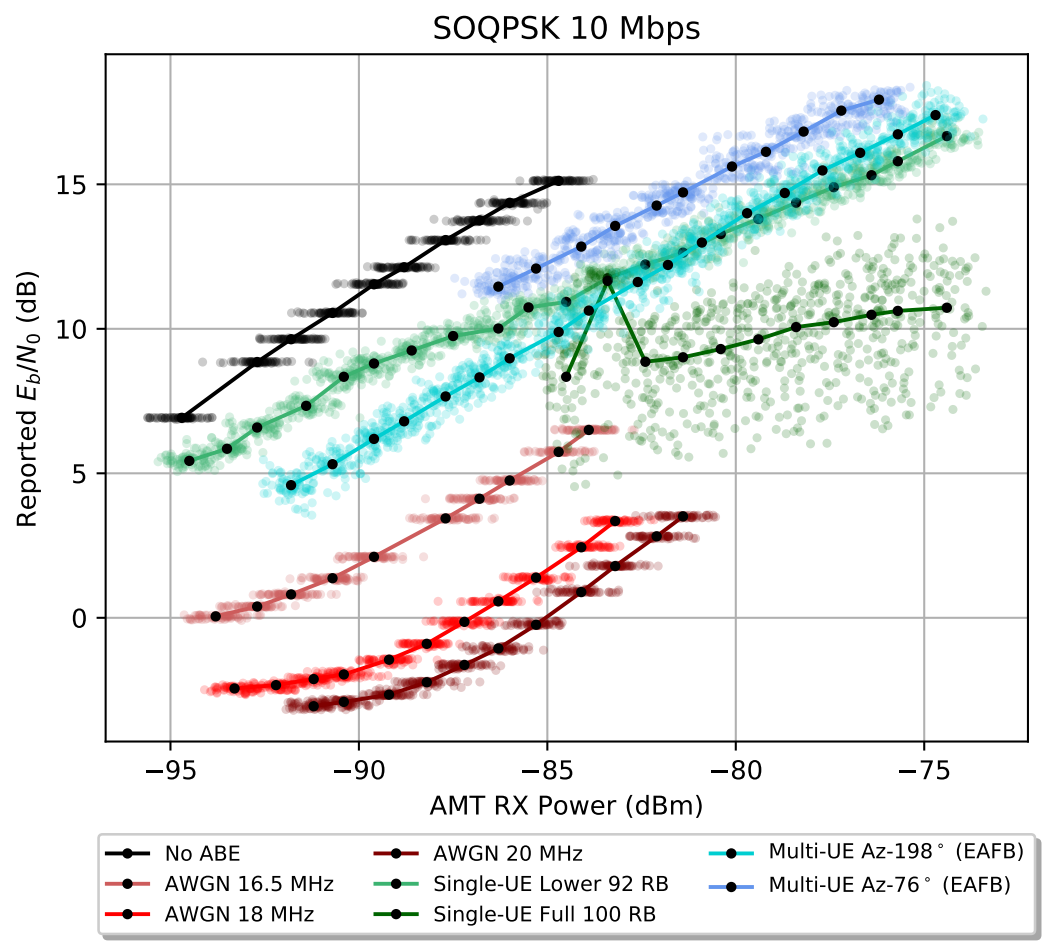

Figure D.4: Reported $E_{b} / N_{0}$ vs AMT RX power for select ABE types: SOQPSK 10 Mbps 


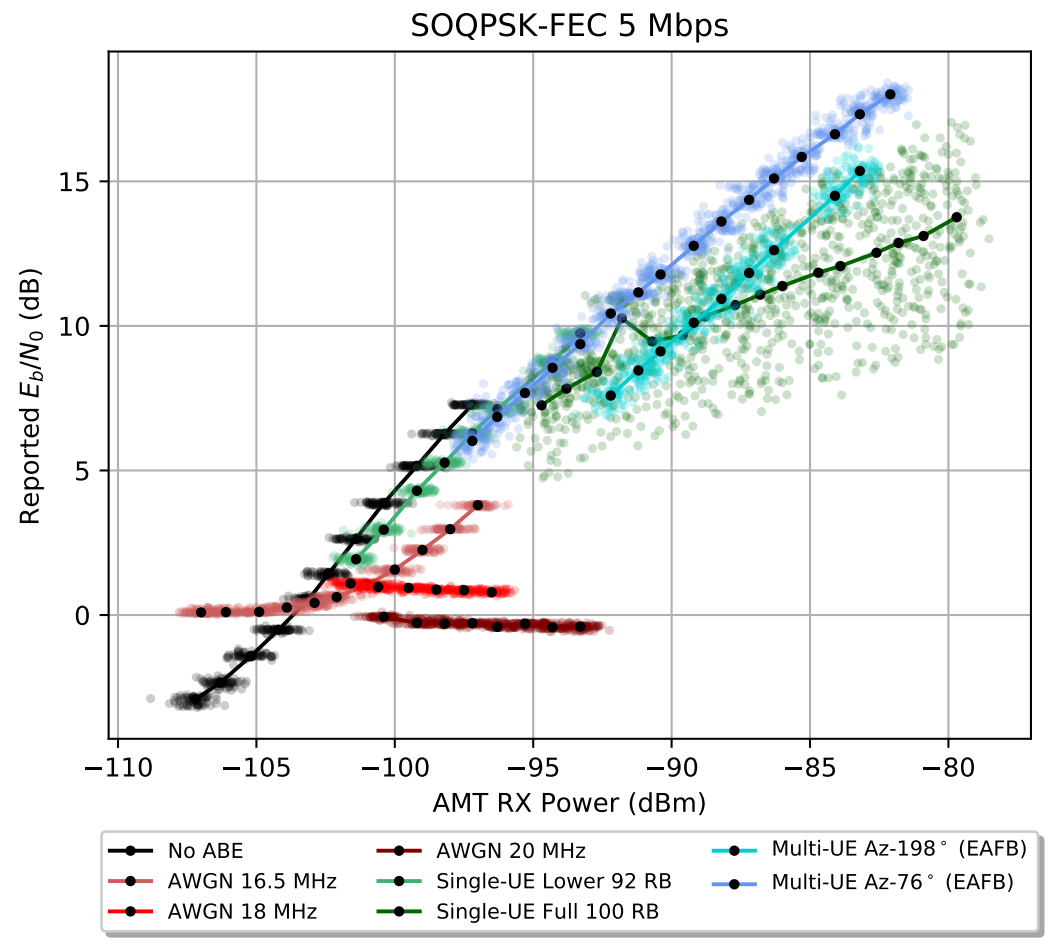

Figure D.5: Reported $E_{b} / N_{0}$ vs AMT RX power for select ABE types: SOQPSK-FEC 5 Mbps

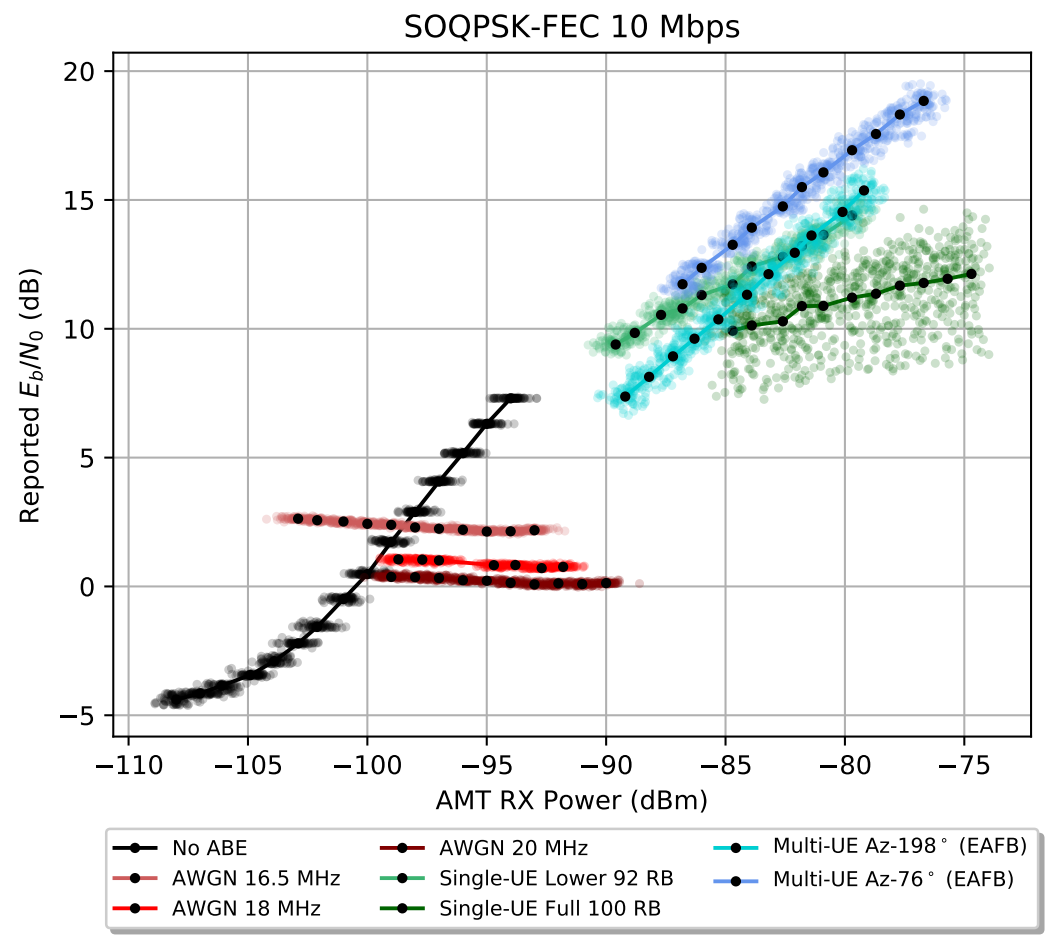

Figure D.6: Reported $E_{b} / N_{0}$ vs AMT RX power for select ABE types: SOQPSK-FEC 10 Mbps 


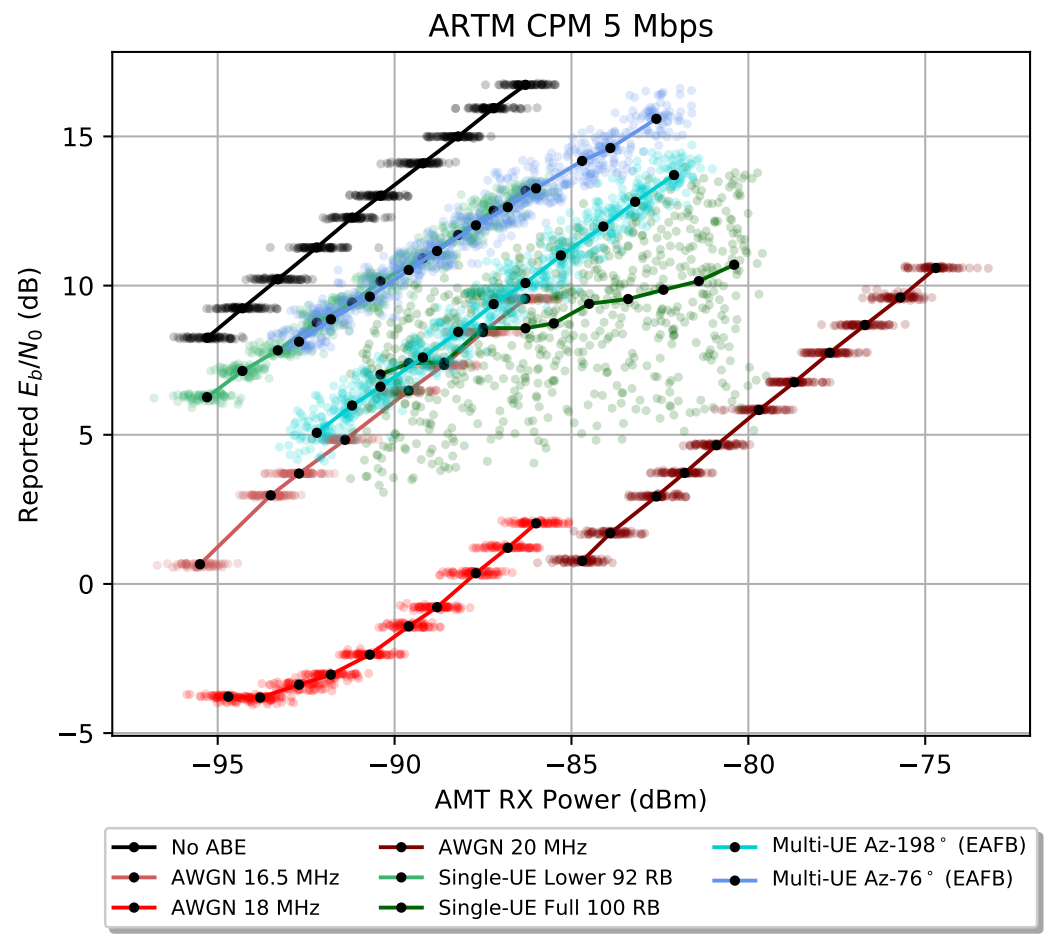

Figure D.7: Reported $E_{b} / N_{0}$ vs AMT RX power for select ABE types: ARTM CPM 5 Mbps 


\section{Appendix E}

\section{BER vs Eb/No in the Baseline no-ABE Case}

Plots of BER versus $E_{b} / N_{0}$ are a traditional way to assess receiver performance. Here, we present such plots for the baseline no-ABE condition in the main experiment as a check on system performance.

For an ideal receiver in an AWGN channel, there is a well-understood theoretical relationship between BER and $E_{b} / N_{0}$ for many commonly-used modulation schemes. Namely, for quadrature-phase shift keying (QPSK) [24, 25],

$$
\mathrm{BER}=Q\left(\sqrt{2 \frac{E_{b}}{N_{0}}}\right)=\frac{1}{2} \operatorname{erfc}\left(\sqrt{\frac{E_{b}}{N_{0}}}\right),
$$

where $Q(\cdot)$ is the complementary cumulative distribution function (CDF) of the standard normal distribution, and $\operatorname{erfc}(\cdot)$ is the complementary error function. As noted by Simon [28], the above relationship also holds for offset quadrature-phase shift keying (OQPSK), a close relative of SOQPSK. For PCM/FM, a similar optimal result given by Punnoose [29] is

$$
\mathrm{BER}=Q\left(\sqrt{\frac{4.86}{2} \frac{E_{b}}{N_{0}}}\right)=\frac{1}{2} \operatorname{erfc}\left(\sqrt{1.22 \frac{E_{b}}{N_{0}}}\right) .
$$

Below, for the priority configurations in the main experiment, BER is plotted against $E_{b} / N_{0}$ at the input to the receiver and the receiver-reported $E_{b} / N_{0}$. For PCM/FM and SOQPSK cases, the ideal theoretical curves given above are also plotted for reference. In linear units, $E_{b} / N_{0}$ at the input to the receiver is calculated by dividing the AMT signal power at the plane of the receiver by the bit-rate to get $E_{b}$, and then dividing by the thermal noise floor, $N_{0}=-174.0 \mathrm{dBm} / \mathrm{Hz}$.

In addition, each figure contains a plot comparing median receiver-reported $E_{b} / N_{0}$ to $E_{b} / N_{0}$ at the receiver input. Since receiver-reported $E_{b} / N_{0}$ is estimated at an unknown plane inside the receiver, we expect these values to be approximately offset by the noise figure of the receiver. 

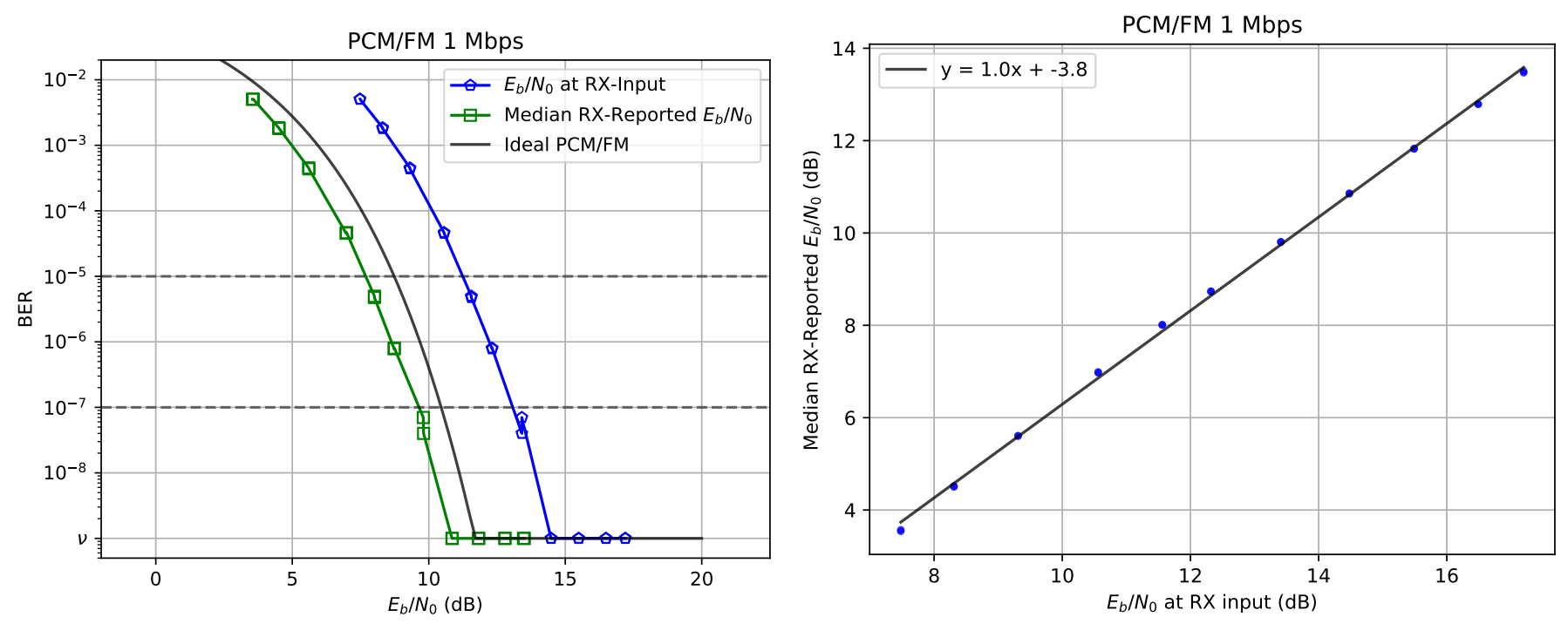

Figure E.1: BER vs. RX-Reported $E_{b} / N_{0}$ and $E_{b} / N_{0}$ at RX-Input: PCM/FM 1 Mbps with No ABE
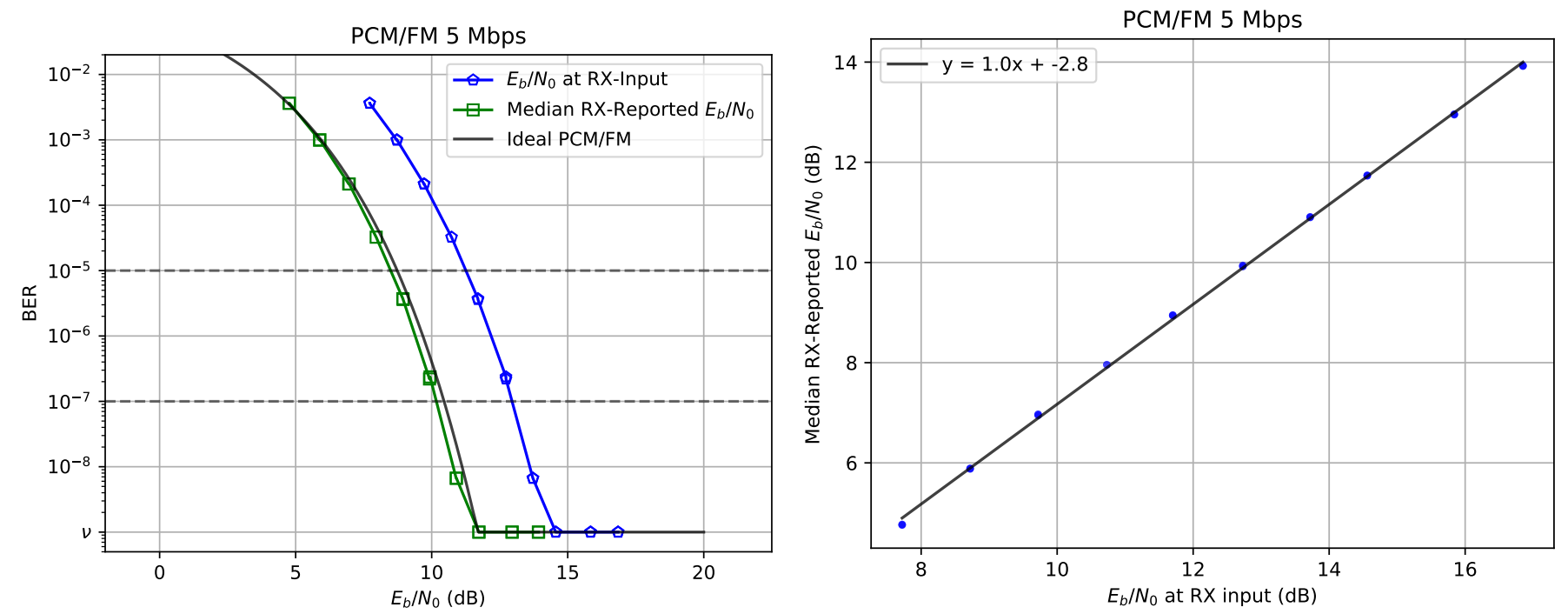

Figure E.2: BER vs. RX-Reported $E_{b} / N_{0}$ and $E_{b} / N_{0}$ at RX-Input: PCM/FM 5 Mbps with No ABE 

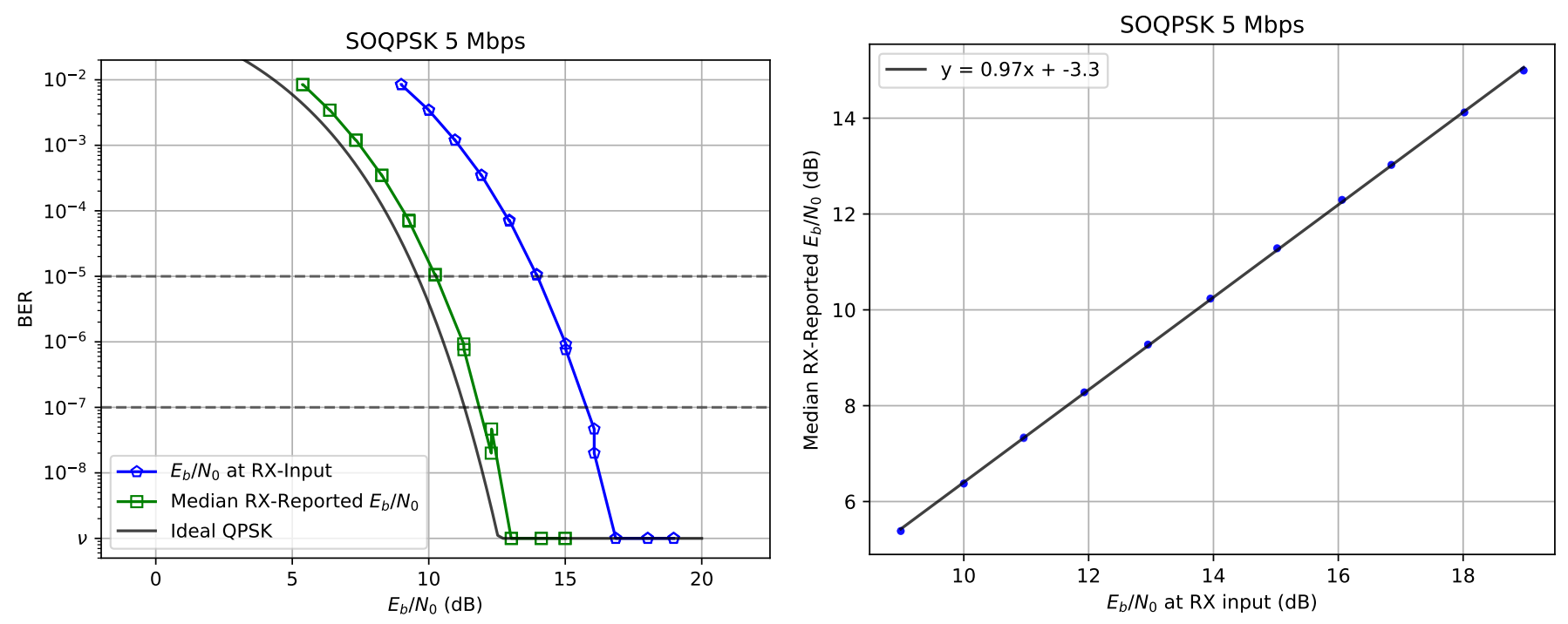

Figure E.3: BER vs. RX-Reported $E_{b} / N_{0}$ and $E_{b} / N_{0}$ at RX-Input: SOQPSK 5 Mbps with No ABE
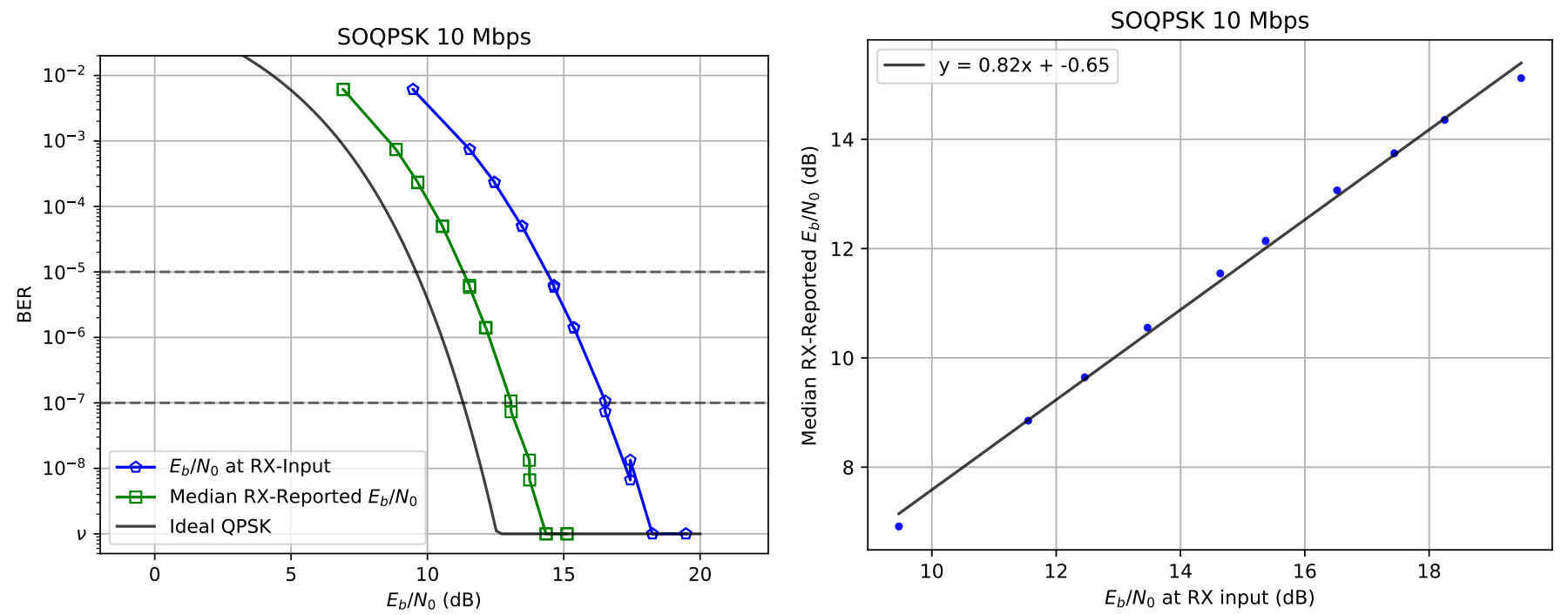

Figure E.4: BER vs. RX-Reported $E_{b} / N_{0}$ and $E_{b} / N_{0}$ at RX-Input: SOQPSK 10 Mbps with No ABE 

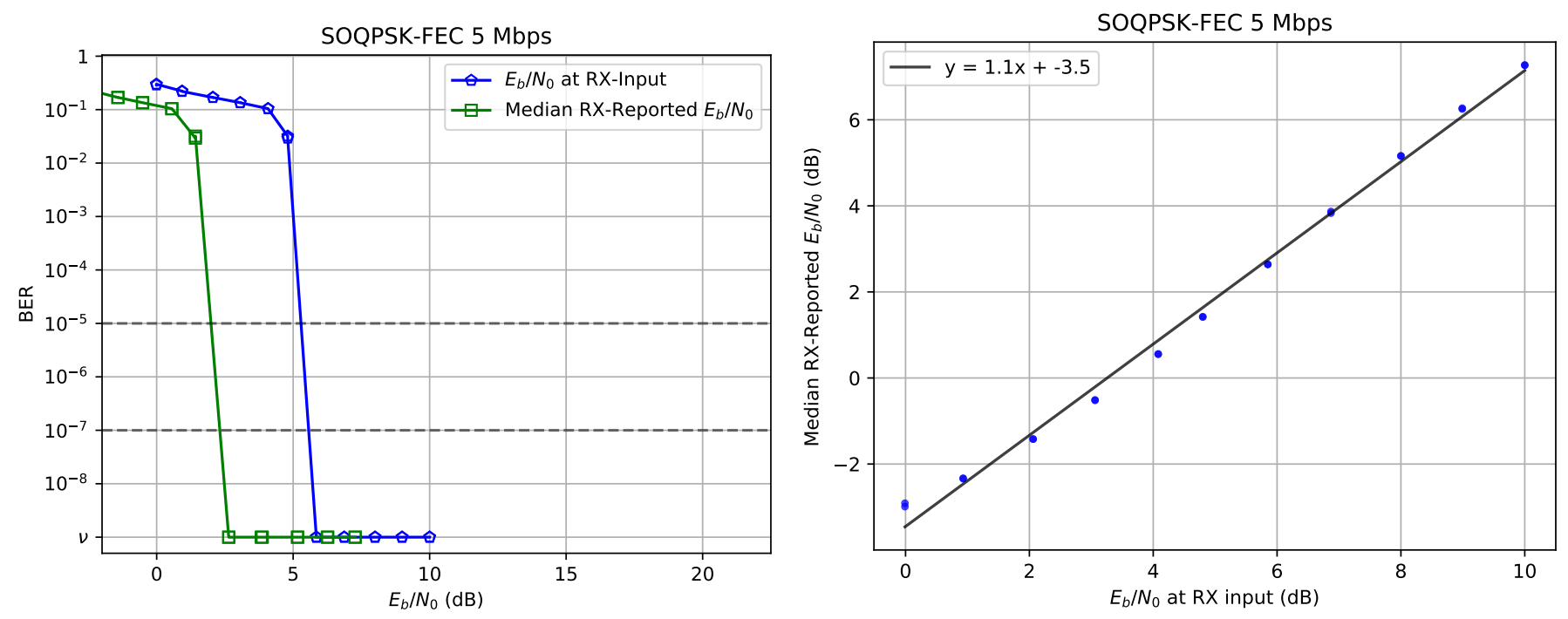

Figure E.5: BER vs. RX-Reported $E_{b} / N_{0}$ and $E_{b} / N_{0}$ at RX-Input: SOQPSK-FEC 5 Mbps with No ABE
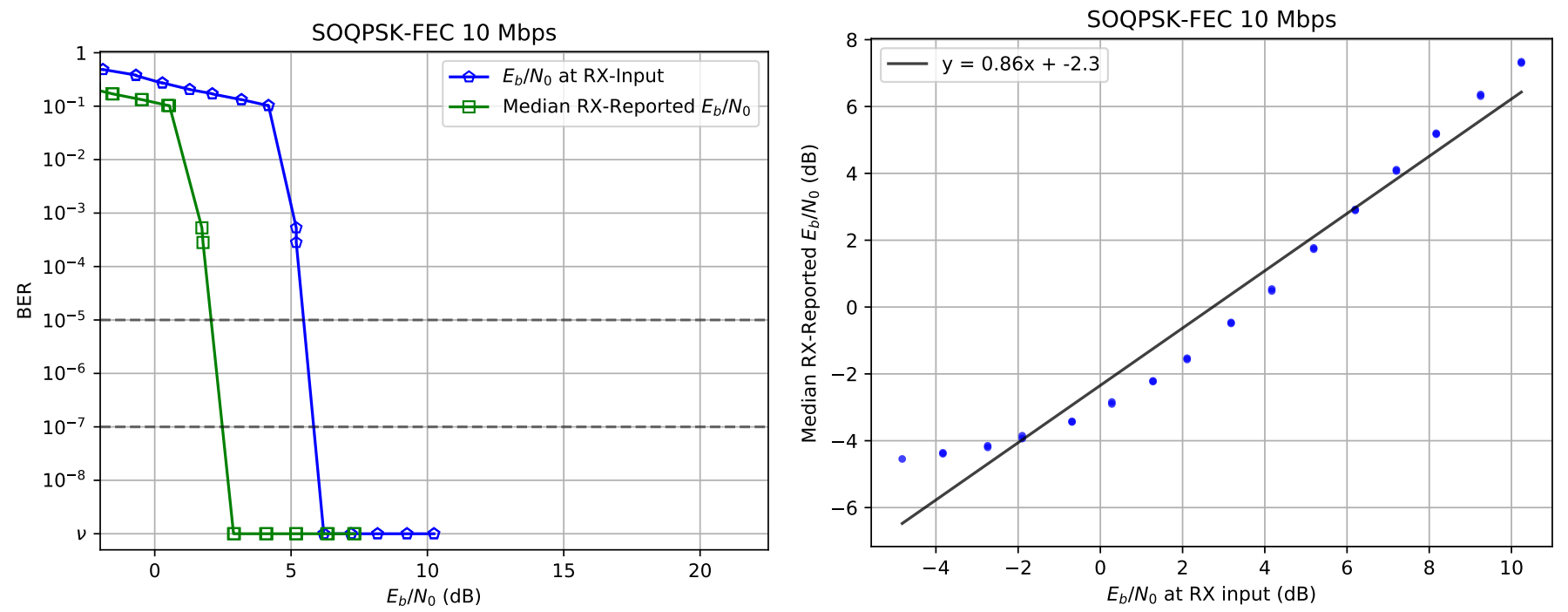

Figure E.6: BER vs. RX-Reported $E_{b} / N_{0}$ and $E_{b} / N_{0}$ at RX-Input: SOQPSK-FEC $10 \mathrm{Mbps}$ with No ABE 

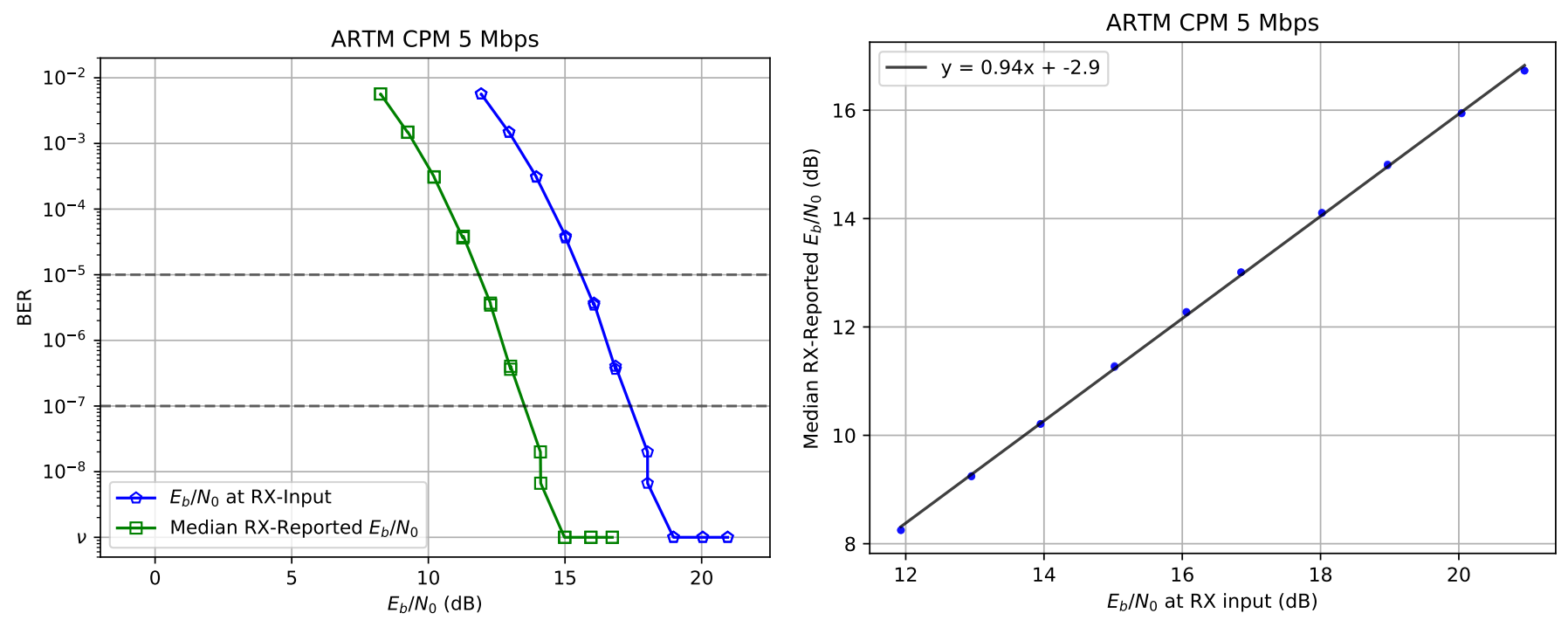

Figure E.7: BER vs. RX-Reported $E_{b} / N_{0}$ and $E_{b} / N_{0}$ at RX-Input: ARTM CPM 5 Mbps with No ABE 


\section{Appendix F}

\section{AMT Receiver Noise Figure}

The noise figure (NF) of the receiver under test characterizes the reduction in $E_{b} / N_{0}$ attributable to thermal noise in the receiver. This is an auxiliary parameter needed to model separable sources of noise in the receiver.

The NF approximation given by the the linear regression offset on (Figs. E.1 - E.7) is an effective way to correct for the physical differences between the $E_{b} / N_{0}$ input conditions and RX-reported $E_{b} / N_{0}$. A physical NF measurement helps to ensure that (a) any differences in the receiver paths that produce the RX-reported $E_{b} / N_{0}$ compared to the symbol decoding process are included, and (b) errors from any bias or non-linearity in the RX-reported $E_{b} / N_{0}$ are mitigated. The measurement also serves as a verification of the NF specification given by the receiver manufacturer.

Receiver response data like BER or RX-reported $E_{b} / N_{0}$ with possibly non-linear and/or biased response to input $E_{b} / N_{0}$ are supported by a new NF measurement technique that was recently developed at NIST. We follow this procedure, which is specified in [30]. The approach is to assess any SNR-dependent receiver output across randomized levels of both incident AMT signal and incident excess noise calibrated at the receiver input. A post-analysis regression identifies the additive noise power that aligns the receiver output with and without excess noise. The regression result is determined relative to the calibrated levels of excess noise, and can therefore be traceable to physical noise standards. The NF measurement result can be thought of as the equivalent incident AWGN required to reproduce all additive noise inside the receiver.

The NF measurement results are given by Table F.1. The AMT signaling conditions tested are PCM/FM 1 Mbps and SOQPSK $10 \mathrm{Mbps}$, in order to cover the bandwidth extrema of the principal AMT modulation schemes under test. Measurements are shown based on separate analysis of the same data in based on perturbing (a) the BER measurement result that is the main subject of this report as well as (b) the RX-reported $E_{b} / N_{0}$. These are both valid data sources for blind NF measurement, because we expect these outputs to respond as functions of SNR. In order to maximize the sensitivity of the measurement, only the subset of data at which BER $<10^{-1}$ were used.

The given intervals validate the measurement by overlapping across all tested conditions RX outputs. The $0.4 \mathrm{~dB}$ halfwidths are dominated by the reference noise diode calibration. This dominant uncertainty is caused by interpolating between calibration data provided at $1 \mathrm{GHz}$ and $2 \mathrm{GHz}$ frequency points. It is unclear whether the NF interval specified by the manufacturer also includes measurement uncertainty. However, a degree of cross-validation was achieved here, since the uncertainty intervals of all measurements overlapped with specification except the near-miss with SOQPSK 10 Mbps BER.

Table F.1: AMT Receiver Noise Figure

\begin{tabular}{cccc} 
& \multicolumn{2}{c}{ Measured Noise Figure (dB) } & Specified Noise Figure (dB) \\
AMT Signaling & From BER & From RX-reported $E_{b} / N_{0}$ & By manufacturer \\
\hline PCM/FM 1 Mbps & $3.4 \pm 0.4$ & $3.4 \pm 0.4$ & 3.5 to 5 \\
SOQPSK 10 Mbps & $2.9 \pm 0.4$ & $3.3 \pm 0.4$ & 3.5 to 5 \\
\hline
\end{tabular}




\section{Appendix G}

\section{Test Run Parameters and Key Performance Indicators}

The measurement campaign acquired and paired data outputs from the units within the testbed as outlined in Section 3.7.3. The following table outlines the numerous test run parameters and AMT KPIs that are compiled in our data product.

Table G.1: List of Test Run parameters and AMT KPIs as reported in the test summary files

\begin{tabular}{|c|c|c|c|c|}
\hline Class & Source & Field Name & Description & Additional Notes \\
\hline Test Run & Exp. Design & wp id & Whole-plot identifier & Specifies experimental configuration \\
\hline Test Run & Exp. Design & Repeat Number & Repeat number & \\
\hline Test Run & Exp. Design & Test Number & Test execution order number & \\
\hline Test Run & Testbed & Test Start & Test start time & \\
\hline Test Run & Testbed & Test End & Test end time & \\
\hline Test Run & AMT RX & amtrx bitrate & AMT RX-reported bit rate (Mbps) & \\
\hline Test Run & AMT RX & amtrx mode & AMT RX-reported modulation type & \\
\hline Test Run & VSG & abe waveform & Adjacent band emission waveform type & \\
\hline KPI & Test Analysis & abe peak power & $\begin{array}{l}\text { Ajdacent band emission waveform peak } \\
\text { power at Rx-input }(\mathrm{dBm})\end{array}$ & \\
\hline KPI & Test Analysis & abe RMS power & $\begin{array}{l}\text { Ajdacent band emission waveform RMS } \\
\text { power at Rx-input }(\mathrm{dBm})\end{array}$ & \\
\hline Test Run & VSG & abe frequency & $\begin{array}{l}\text { Set adjacent band emission waveform cen- } \\
\text { ter frequency }(\mathrm{Hz})\end{array}$ & \\
\hline Test Run & Testbed & amt attenuation & AMT Signal Attenuation (dB) & Testbed attenuator value for AMT signal. \\
\hline Test Run & Testbed & abe attenuation & ABE Signal Attenuation (dB) & $\begin{array}{l}\text { Does not include calibrated pathloss. } \\
\text { Testbed attenuator value for ABE. Does } \\
\text { not include calibrated pathloss. }\end{array}$ \\
\hline Test Run & Test Analysis & rx power & $\begin{array}{l}\text { AMT Signal power at the receiver input } \\
(\mathrm{dBm})\end{array}$ & $\begin{array}{l}\text { Estimated from testbed calibration mea- } \\
\text { surements. }\end{array}$ \\
\hline
\end{tabular}




\begin{tabular}{|c|c|c|c|c|}
\hline \multicolumn{5}{|c|}{ - continued from previous page } \\
\hline Class & Source & Field Name & Description & Additional Notes \\
\hline KPI & AMT RX & amtrx Bits & AMT bits received & $\begin{array}{l}\text { Cumulative sum of bits received by AMT } \\
\text { reciever }\end{array}$ \\
\hline KPI & AMT RX & amtrx Errors & AMT bit errors & $\begin{array}{l}\text { Cumulative sum of errors received by } \\
\text { AMT reciever }\end{array}$ \\
\hline KPI & Test Analysis & estimated BER & Estimated Bit error rate (BER) & Calculated as bit errors divided by bits \\
\hline KPI & Test Analysis & $\mathrm{Eb}$ & $\begin{array}{l}\text { Calculated energy per bit at the receiver in- } \\
\text { put }\end{array}$ & $\begin{array}{l}\text { Calculated as AMT signal power ( } \mathrm{rx} \\
\text { power) divided by bit rate (amtrx bitrate) }\end{array}$ \\
\hline Test Run & Testbed & noise diode & Noise Diode on status (True/False) & \\
\hline Test Run & AMT RX & amtrx frequency & Frequency of AMT RX component & \\
\hline KPI & AMT RX & amtrx FPGATemp & Temperature of AMT RX FPGA & \\
\hline KPI & AMT RX & amtrx DemodTemp & Temperature of AMT RX Demodulator & \\
\hline KPI & AMT RX & amtrx InputdBm & Receiver estimated Incident Power & \\
\hline KPI & AMT RX & amtrx AttendB & Internal Attenuator & \\
\hline KPI & AMT RX & amtrx BulkAttenuation & Internal Combined Attenuation & \\
\hline KPI & AMT RX & amtrx SignaldBm & $\begin{array}{l}\text { Receiver estimated demod signal power } \\
(\mathrm{dBm})\end{array}$ & \\
\hline KPI & AMT RX & amtrx AdjusteddBm & Attenuator adjusted signal level & \\
\hline KPI & AMT RX & amtrx EbNOdB & Receiver estimated Eb/N0 (dB) & \\
\hline KPI & AMT RX & amtrx SatCount & Saturation Count & \\
\hline KPI & AMT RX & amtrx DSPSNR & Digital Signal Processor SNR & \\
\hline KPI & AMT RX & amtrx AGCSNR & Automatic Gain Control SNR & \\
\hline KPI & AMT RX & amtrx AFCDetectOffKhz & $\begin{array}{l}\text { Automatic Frequency Control offset in } \\
\mathrm{kHz}\end{array}$ & \\
\hline KPI & AMT RX & amtrx AFCMode & Automatic Frequency Control mode & Off, Hold, Track, or Acquire \\
\hline KPI & AMT RX & amtrx AFCCompensatekHz & The AFC compensation applied & \\
\hline KPI & AMT RX & amtrx AGCEntZero & Automatic Gain Control Entered zero & \\
\hline KPI & AMT RX & amtrx AGCIntZero & Automatic Gain Control internal zero & \\
\hline KPI & AMT RX & amtrx BitrateMbps & Receiver estimated Bitrate of the AMT link & \\
\hline KPI & AMT RX & amtrx Lock & $\begin{array}{l}\text { Boolean if AMT RX met the lock require- } \\
\text { ment }\end{array}$ & \\
\hline KPI & AMT RX & amtrx Enabled & Boolean if Bit Error Rate Test is enabled & \\
\hline KPI & AMT RX & amtrx Running & $\begin{array}{l}\text { Bit indicates that the test is currently run- } \\
\text { ning }\end{array}$ & \\
\hline
\end{tabular}




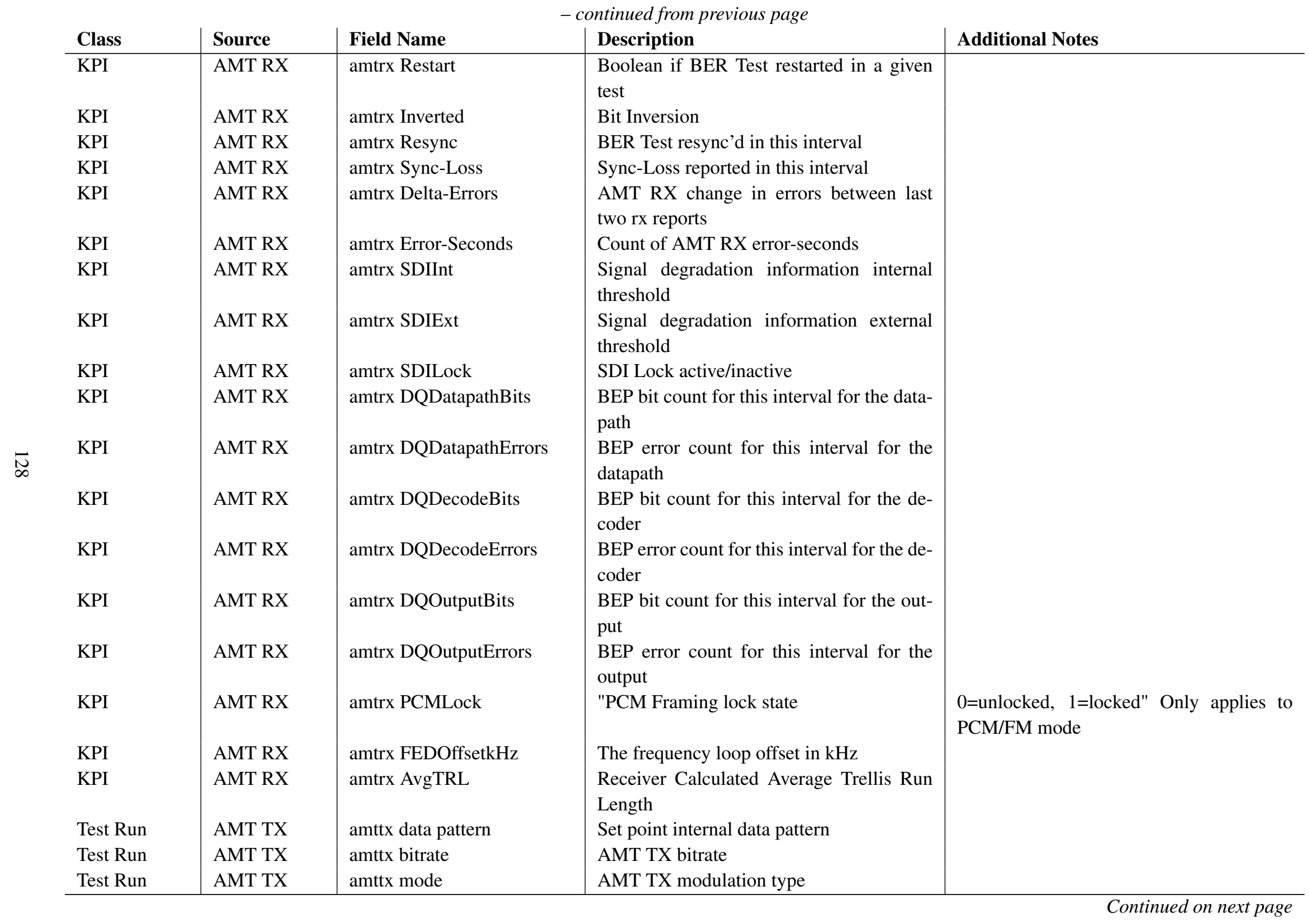




\begin{tabular}{|c|c|c|c|c|}
\hline \multicolumn{5}{|c|}{ - continued from previous page } \\
\hline Class & Source & Field Name & Description & Additional Notes \\
\hline $\begin{array}{l}\text { Test Run } \\
\text { Test Run }\end{array}$ & $\begin{array}{l}\text { AMT TX } \\
\text { AMT TX }\end{array}$ & $\begin{array}{l}\text { amttx frequency } \\
\text { amttx output power }\end{array}$ & $\begin{array}{l}\text { Frequency of AMT TX component } \\
\text { Output power set point of amttx }\end{array}$ & \\
\hline
\end{tabular}




\section{Bibliography}

[1] Inter-Range Instrumentation Group: Range Commanders Council Telemetry Group. IRIG 106-19: Telemetry Standards. [Online]. Available: http://www.irig106.org/docs/106-19/106-19_Telemetry_Standards.pdf

[2] E. D. Nelson and D. A. McGillivray, "In-Situ Captures of LTE for Aeronautical Mobile Telemetry System Evaluation," National Telecommunications and Information Administration, Tech. Rep. in preparation, 2021.

[3] U.S. Government Publishing Office. (2010) Presidential memorandum: Unleashing the wireless broadband revolution. [Online]. Available: https://www.gpo.gov/fdsys/pkg/CFR-2011-title3-vol1/pdf/ CFR-2011-title3-vol1-other-id236.pdf

[4] National Telecommunications and Information Administration. An assessment of the near-term viability of accommodating wireless broadband systems in the $1675-1710 \mathrm{MHz}, 1755-1780 \mathrm{MHz}, 3500-3650 \mathrm{MHz}$, and 4200-4220 MHz, 4380-4400 MHz bands. [Online]. Available: https://www.ntia.doc.gov/files/ntia/publications/ fasttrackevaluation_11152010.pdf

[5] Federal Communications Commission. Auction 97, Advanced wireless services (AWS-3). [Online]. Available: https://www.fcc.gov/auction/97

[6] M. Frey, G. Sanders, J. Splett, J. Ladbury, F. Sanders, A. Kord, and R. Jacobs, "Measured Emission Spectra of Selected AWS-3 LTE Transmitters," National Institute of Standards and Technology, Tech. Rep. 1980, Dec 2017. [Online]. Available: https://doi.org/10.6028/NIST.TN.1980

[7] S. M. Kowalski, P. A. Parker, and G. G. Vining, “Tutorial: Industrial split-plot experiments," Quality Engineering, vol. 19, no. 1, pp. 1-15, 2007.

[8] D. C. Montgomery, Design and Analysis of Experiments, 9th ed. Hoboken, NJ: John Wiley \& Sons, 2017.

[9] C. F. Wu and M. S. Hamada, Experiments: Planning, Analysis, and Optimization, 2nd ed. Hoboken, NJ: John Wiley \& Sons, 2009.

[10] A. W. Sanders, M. K. Forsyth, F. C. da Silva, R. D. Horansky, A. Kord, and D. A. McGillivray, "Laboratory Method for Recording AWS-3 LTE Waveforms," National Institute of Standards and Technology, Tech. Rep. in preparation, 2021.

[11] National Telecommunications and Information Administration, "Manual of regulations and procedures for federal radio frequency management," 2017. [Online]. Available: https://www.ntia.doc.gov/page/2011/ manual-regulations-and-procedures-federal-radio-frequency-management-redbook

[12] Inter-Range Instrumentation Group: Range Commanders Council Telemetry Group. IRIG 118-12: Telemetry Standards Test Methods for Vehicle Telemetry Systems. [Online]. Available: https://www.wsmr.army.mil/ RCCsite/Pages/Publications.aspx

[13] Quasonix, Installation and Operation Manual: 3rd Generation, Rack-Mount RDMS Telemetry Receiver, April 2019, version 3.4.5. 
[14] L. D. Brown, T. T. Cai, and A. DasGupta, "Interval estimation for a binomial proportion," Statistical Science, pp. 101-117, 2001.

[15] A. DasGupta, Fundamentals of Probability: A First Course. S Springer, 2010.

[16] W. Feller, "On the normal approximation to the binomial distribution," in Selected Papers I. Springer, 2015, pp. $655-665$.

[17] C. J. Clopper and E. S. Pearson, "The use of confidence or fiducial limits illustrated in the case of the binomial," Biometrika, vol. 26, no. 4, pp. 404-413, 1934.

[18] W. S. Cleveland, "Robust locally weighted regression and smoothing scatterplots," Journal of the American Statistical Association, vol. 74, no. 368, pp. 829-836, 1979.

[19] K. Takezawa, Introduction to Nonparametric Regression. Hoboken, NJ: John Wiley \& Sons, 2006.

[20] D. I. Warton and F. K. Hui, "The arcsine is asinine: the analysis of proportions in ecology," Ecology, vol. 92, no. 1, pp. 3-10, 2011.

[21] H. Kibirige, "Locally-weighted regression," 2016. [Online]. Available: https://has2k1.github.io/scikit-misc/ loess.html

[22] W. S. Cleveland, E. Grosse, and M.-J. Shyu, "Software for locally-weighted regression," 1992. [Online]. Available: https://www.netlib.org/a/dloess

[23] G. Casella and R. L. Berger, Statistical Inference, 2nd ed. Duxbury, 2001.

[24] J. G. Proakis and M. Salehi, Communication Systems Engineering. Prentice Hall, 2002.

[25] A. F. Molisch, Wireless Communications. John Wiley \& Sons, 2012.

[26] M. G. Arthur, “The Measurement of Noise Performance Factors: A Metrology Guide," U.S. National Bureau of Standards, Boulder, CO, Tech. Rep. 142, June 1974.

[27] D. M. Pozar, Microwave Engineering, 3rd ed. John Wiley \& Sons Inc, 2005.

[28] M. K. Simon, Bandwidth-Efficient Digital Modulation with Application to Deep Space Communications. John Wiley \& Sons, 2003.

[29] R. J. Punnoose, "Re-engineering PCM/FM as a phase modulation scheme." Sandia National Laboratory, Livermore, CA, Tech. Rep. SAND2008-3557C, 2008.

[30] D. G. Kuester, A. Wunderlich, D. A. McGillivray, D. Gu, and A. K. Puls, "Blind measurement of receiver system noise," IEEE Transactions on Microwave Theory and Techniques, vol. 68, no. 6, pp. 2435-2453, 2020. 


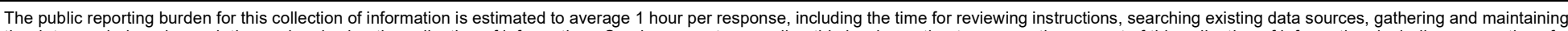

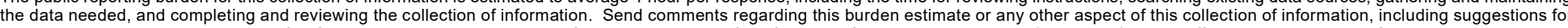

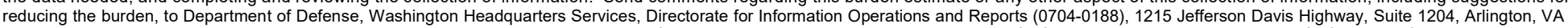

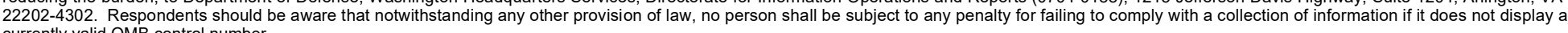
currently valid OMB control number.

PLEASE DO NOT RETURN YOUR FORM TO THE ABOVE ADDRESS.

\begin{tabular}{|c|r|r|}
\hline $\begin{array}{c}\text { 1. REPORT DATE }(D D-M M-Y Y Y Y) \\
\text { 24-02-2021 }\end{array}$ & $\begin{array}{l}\text { 2. REPORT TYPE } \\
\text { NIST Technical Note }\end{array}$ & $\mathbf{2 4 - 0 2 - 2 0 1 8 - 0 1 - 0 1 - 2 0 2 1}$ \\
\hline
\end{tabular}

4. TITLE AND SUBTITLE

AWS-3 LTE Impacts on Aeronautical Mobile Telemetry

5a. CONTRACT NUMBER

F1S0AG8229G001

5b. GRANT NUMBER

5c. PROGRAM ELEMENT NUMBER

R20-670-0004

5d. PROJECT NUMBER

6702378

5e. TASK NUMBER

000

5f. WORK UNIT NUMBER

30-67-0670-02-00-00-00

7. PERFORMING ORGANIZATION NAME(S) AND ADDRESS(ES)

National Institute of Standards and Technology (NIST)

Communications Technology Laboratory, NASCTN Program Office

325 Broadway

Boulder, CO 80305

9. SPONSORING/MONITORING AGENCY NAME(S) AND ADDRESS(ES)

Edwards Air Force Base

412th Test Engineering Group, Spectrum Relocation Program

307 E Popson Ave, Ste 204

Edwards AFB, CA 93524-1180

12. DISTRIBUTION/AVAILABILITY STATEMENT

Approved for Public Release; Distribution Unlimited.

\section{SUPPLEMENTARY NOTES}

MITRE - Approved for Public Release; Distribution Unlimited. Public Release Case Number 21-0100. Government under contract TIRNO-99-D-00005 and Task Order 1333ND20FNB670406 and is subject to FAR 52.227-17 "Rights in Data - Special Works" clause.

\section{ABSTRACT}

The objective of this NASCTN effort is to design, demonstrate, and validate a test methodology to measure the impacts of LTE UE emissions on the adjacent AMT band. Impacts are assessed by collecting and analyzing performance measures, such as bit error rate (BER) and Eb/N0, generally referred to as key performance indicators (KPIs). The validation process includes the capture and analysis for multiple adjacent band emissions (ABE) and AMT transmission combinations and conditions. The analysis here centers around BER behavior with and without the presence of $A B E$.

\section{SUBJECT TERMS}

AWS-3, LTE, Aeronautical Mobile Telemetry, Susceptibility Testing, Adjacent Band Emissions, RF Testing, Metrology.

\begin{tabular}{|c|c|c|c|}
\hline \multicolumn{2}{|l|}{ 16. SECURITY CLASSIFICATION OF: } & 17. LIMITATION OF \\
ABSTRACT \\
a. REPORT & b. ABSTRACT & c. THIS PAGE & \\
UNCLASSIFIED & UNCLASSIFIED & UNCLASSIFIED & None
\end{tabular}

18. NUMBER 19a. NAME OF RESPONSIBLE PERSON

PAGES

Melissa Midzor

19b. TELEPHONE NUMBER (Include area code)

131

(303) 497-3591 